\title{
Analysis of seismo-acoustic emission from ice fracturing events during SIMI'94
}

by

\author{
Yuriy V. Dudko \\ M.S., Electrical Engineering \\ Moscow Institute of Physics and Technology \\ Submitted in partial fulfillment of the \\ requirements for the Degree of
}

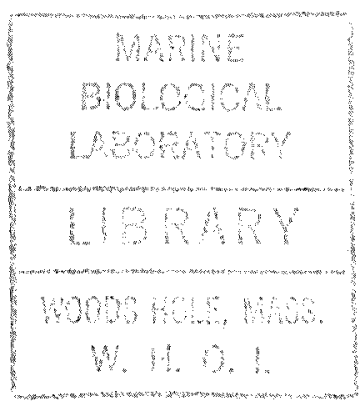

DOCTOR OF PHILOSOPHY IN OCEANOGRAPHIC ENGINEERING

at the

\author{
MASSACHUSETTS INSTITUTE OF TECHNOLOGY \\ and the
}

WOODS HOLE OCEANOGRAPHIC INSTITUTION

February 1999

(C) 1999 Massachusetts Institute of Technology and Woods Hole Oceanographic

Institution

All rights reserved

Autnu.

:n Applied Ocean Science and Engineering -achusetts Institute of Technology

- nceanographic Institution

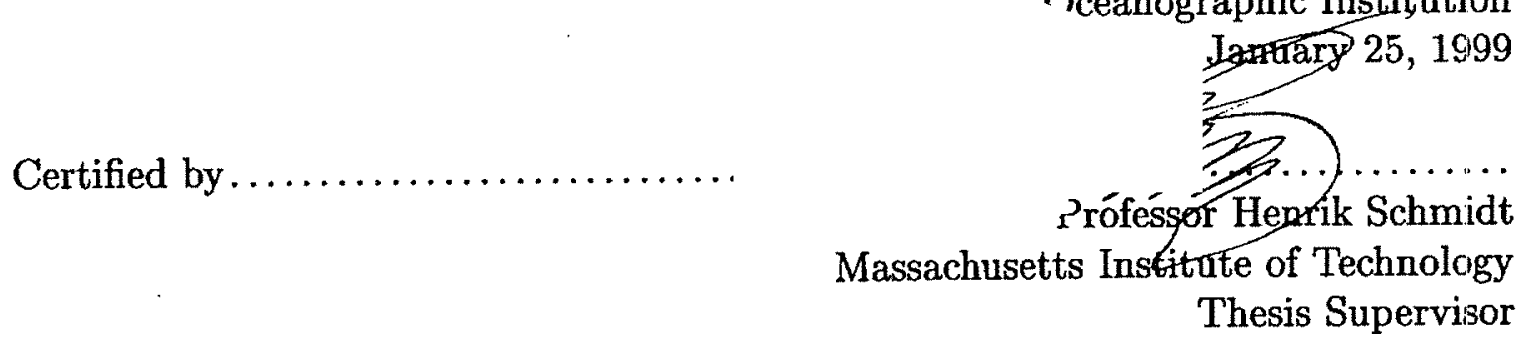

Accepted by .........

Professor Michael Triantafyllou

Chairman, Joint Committee for Applied Ocean Science and Engineering Massachusetts Institute of Technology/Woods Hole Oceanographic Institution 


\title{
Analysis of seismo-acoustic emission from ice fracturing events during SIMI'94
}

\author{
by \\ Yuriy V. Dudko \\ Submitted to the Massachusetts Institute of Technology/ \\ Woods Hole Oceanographic Institution \\ Joint Program in Applied Ocean Science and Engineering on January 25, 1999 in \\ partial fulfillment of the \\ requirements for the Degree of \\ DOCTOR OF PHILOSOPHY IN OCEANOGRAPHIC ENGINEERING
}

\section{Abstract}

In this thesis the analysis of natural ice events is carried out based on direct measurements of ice-borne seismo-acoustic waves generated by ice fracturing processes. A major reason for studying this phenomenon is that this acoustic emission is a significant contributor to Arctic ocean ambient noise. Also the Arctic contains rich mineral and oil resources and in order to design mining facilities able to withstand the harsh environmental conditions, we need to have a better understanding of the processes of sea ice mechanics. The data analyzed in this thesis were collected during the Sea Ice Mechanics Initiative (SIMI'94) experiment which was carried out in the spring of 1994 in the Central Arctic.

One of the contributions of this thesis was the determination of the polarization characteristics of elastic waves using multicomponent geophone data. Polarization methods are well known in seismology, but they have never been used for ice event data processing. In this work one of the polarization methods (so called Motion Product Detector method) has been successfully applied for localization of ice events and determination of polarization characteristics of elastic waves generated by fracturing events. This application demonstrates the feasibility of the polarization method for ice event data processing because it allows one to identify areas of high stress concentration and "hot spots" in ridge building process.

The identification of source mechanisms is based on the radiation patterns of the events. This identification was carried out through the analysis of the seismo-acoustic emission of natural ice events in the ice sheet. Previous work on natural ice event identification was done indirectly by analyzing the acoustic energy radiated into the water through coupling from elastic energy in the ice sheet.

After identification of the events, the estimation of the parameters of fault processes in Arctic ice is carried out. Stress drop, seismic moment and the type of ice fracture are determined using direct near-field measurements of seismo-acoustic signals generated by ice events. Estimated values of fracture parameters were in good 
agreement with previous work for marginal ice zone.

During data processing the new phenomenon was discovered: "edge waves", which are waves propagating back and forth along a newly opened ice lead. These waves exhibit a quasi-periodic behavior suggesting some kind of stick-slip generation mechanism somewhere along the length of the lead. The propagation characteristics of these waves were determined using seismic wavenumber estimation techniques. In the lowfrequency limit the dispersion can be modeled approximately by an interaction at the lead edges of the lowest order, antisymmetric modes of the infinite plate.

Thesis Supervisor: Henrik Schmidt

Title: Professor, Massachusetts Institute of Technology 


\section{Acknowledgments}

First of all, I would like to thank my advisor, Prof. Henrik Schmidt. He was always there when I needed his guidance. I deeply appreciate his deep physical insight and technical expertise which helped me a lot in my research. He is a great scientist and a mentor.

I want also to thank the rest of my thesis committee, Prof. Arthur Baggeroer, Dr. James Lynch and Dr. William M. Carey, for all the indispensable help they provided during my thesis research. I would like to thank Prof. Ira Dyer for his valuable comments during my thesis defense.

Thanks for help in working with experimental data from SIMI experiment go to Edward K. Scheer and Keith von der Heydt of Woods Hole Oceanographic Institution. Without them the data set around which this thesis revolves simply would not exist.

I am also very grateful to the Acoustics group administrative staff, especially Sabina Rataj. Her caring made my life at MIT much more pleasant. Thank you, Sabina. Thanks also to Robert Fadel, Patrick Preston, and Isela Cordova.

Two courses I have taken at MIT stand out in my memory. The first one is 6.432 "Stochastic Processes, Detection, and Estimation" taught by Prof. Gregory Wornell. $\mathrm{He}$ is a great teacher able to deliver very complicated concepts of signal processing theory with crystal clarity. The second course is 1.124 "Computer Aided Engineering" taught by Prof. John Williams. It was a great course and a wonderful experience. I learned a lot through the lectures and the team project.

I want to give my thanks to Prof. Nafi Toksoz of MIT ERL for his help with earthquake seismology.

Support for this thesis was provided by Office of Naval Research, it is most gratefully acknowledged.

This work would not be possible without the help of my fellow students in the Acoustics Group throughout the years. I would like to thank Jo-Tiam (JT) Goh for introducing me into the wonderful world Linux and for his technical advice. Thanks to Peter Daly for all his help with computer problems. 
Vincent Lupien and Eugene Dorfman were more than just office-mates; they became real friends. I will never forget our discussions. Kyle Becker, Trym Eggen, Brian Sperry, Brian Tracey, Jaiyong Lee, Dan Li, Yi-San Lai, Gongwen Xu, Joseph Bondaryk, Ken Rolt, Dong Guk Paeng, Tarun Kapoor, Jeong-Ho Park and the rest of 5-007 and 5-435 crew, thanks for your company.

I want to give special thanks to Vincent Lupien, Pierre Elisseeff, Jaiyong Lee and Brian Sperry for help in preparing my thesis defense presentation, and to Vincent Lupien, Brian Sperry, Pierre Elisseeff and Dianne Egnor for reading my thesis and making valuable comments.

Last but not the least I want to thank my family, wife Eugenia and son Roman, for all their help and support during my thesis research. 


\section{Contents}

1 Introduction $\quad 18$

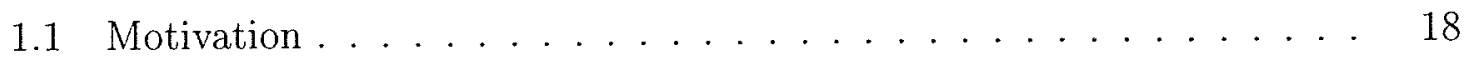

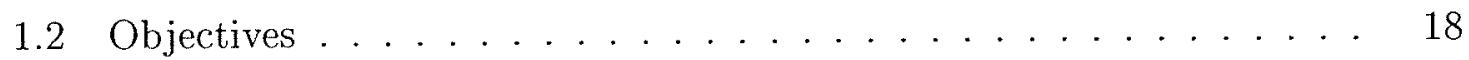

1.3 Previous work ......................... 20

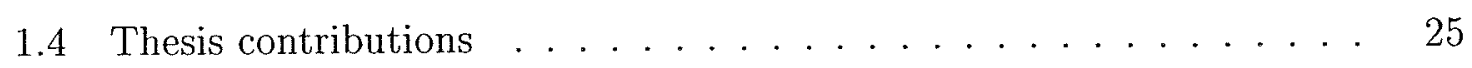

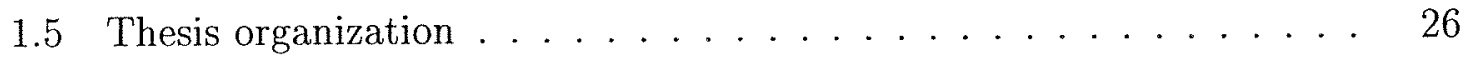

2 Sea Ice Mechanics Initiative (SIMI) Experiment overview and data $\begin{array}{ll}\text { preparation procedure } & 27\end{array}$

2.1 Overview of the SIMI experiment . . . . . . . . . . . 27

2.2 SIMI arrays and systems . . . . . . . . . . . . . 29

2.2.1 Surveillance Hydrophone Array . . . . . . . . . . . . 29

2.2 .2 Geophone Clusters . . . . . . . . . . . . 33

2.3 Preparation of the SIMI data . . . . . . . . . . . 35

2.3.1 Preprocessing of geophone data . . . . . . . . 35

2.3.2 Event detection procedure . . . . . . . . . . 37

2.3.3 Event localization scheme . . . . . . . . . . 39

2.4 Flexural wave arrival . . . . . . . . . . . . . 50

2.4.1 Multiple filter technique ............ 50

2.4 .2 Flexural wave dispersion ............... 51

2.4.3 Sense of motion in flexural wave ......... 56 
3 Polarization processing $\quad 60$

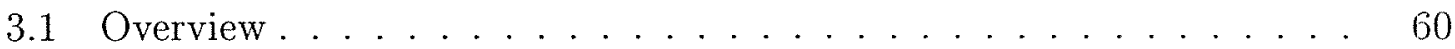

3.2 Motion Product Detector (MPD) seismogram method . . . . . . . 61

3.2.1 Procedure for isolating rectilinear polarization in data recordings by the MPD method ............. . . 63

3.2.2 Procedure for isolating elliptic polarization in data recordings by the MPD method . . . . . . . . . . . . 65

3.3 Data processing scheme ............... 66

3.4 Results of data processing by the MPD method . . . . . . . . . 73

3.4.1 MPD seismograms . . . . . . . . . . . . . . . 79

3.4 .2 Triangulation results .............. 80

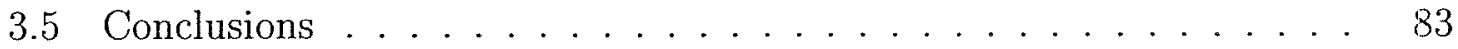

4 Edge waves $\quad 86$

4.1 Overview .............................. 86

4.2 The experimental setup and the geophone data . . . . . . . . . 91

4.3 Data processing scheme . . . . . . . . . . . . . . 92

4.4 Theoretical model . . . . . . . . . . . . . . . . 98

4.4.1 General equations of motion in elastic medium . . . . . . . 98

4.4.2 The fundamental modes of the infinite plate . . . . . . 105

4.4.3 The geometry of the problem . . . . . . . . . . 112

4.4.4 The boundary conditions in the weak form . . . . . . . . 113

4.5 Comparison of model and the data . . . . . . . . . . 117

4.6 Conclusions . . . . . . . . . . . . . . . . . . 118

5 Event Identification $\quad 120$

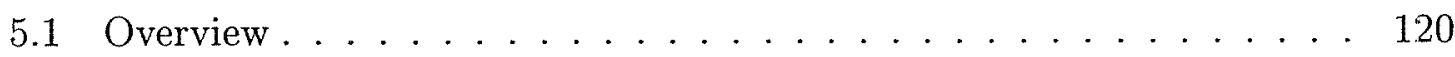

5.2 Fault types definitions . . . . . . . . . . . . . 121

5.2.1 Fault orientation description .............. 121

5.2 .2 Three modes of cracking . . . . . . . . . . 122

5.3 Radiation patterns for different types of ice cracks . . . . . . . 123 
5.3.1 Shear crack radiation patterns . . . . . . . . . . . . 124

5.3 .2 Tensile crack radiation patterns . . . . . . . . . . . 129

5.3.3 Analytical models for crack radiation patterns . . . . . . . 131

5.4 Procedure for fitting analytical radiation patterns to experimental data 132

5.4 .1 Model parameterization . . . . . . . . . . . . 132

5.4.2 Preprocessing of experimental data for determining the radiation patterns . . . . . . . . . . . . 133

5.4.3 Model pattern fitting to experimental points . . . . . . . 134

5.5 Qualitative determination of event mechanisms . . . . . . 135

5.5 .1 Cluster \#1 . . . . . . . . . . . . . . . . . . . 136

5.5.2 Other events at the beginning of tape RLAM-26 . . . . . 140

5.5.3 Events at the beginning of file \#5 of tape RLAM-26 . . . 143

5.6 Conclusions . . . . . . . . . . . . . . . . . . . . . . . . . . . . 151

6 Event parameter estimation $\quad 152$

6.1 Overview . . . . . . . . . . . . . . . . . . . . . . . . . 152

6.2 Frequency domain processing for event parameter estimation . . . . 153

6.2.1 Standard earthquake model of the displacement spectrum . . . 153

6.2.2 Results of corner frequency estimation . . . . . . . . . 154

6.3 Slip history determination $\ldots \ldots \ldots \ldots \ldots \ldots$

6.3.1 Two models for the slip history of the fault . . . . . . . 158

6.3.2 Results of slip history determination . . . . . . . . . 161

6.4 Event parameter estimation from the combined results of spectral processing and slip history determination . . . . . . . . . . 170

6.4.1 Event parameter definitions . . . . . . . . . . . 170

6.4.2 Procedure for event parameter estimation . . . . . . . 172

6.4.3 The results of estimation of event parameters and comparison with previous estimates. . . . . . . . . . . . 173

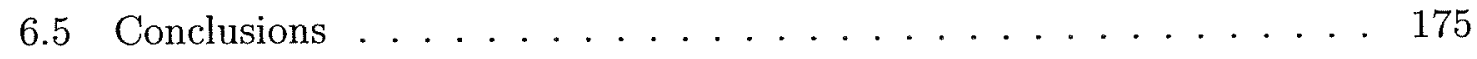


7 Conclusions and suggestions for future work

7.1 Conclusions ........................ 177

7.2 Suggestions for future work . . . . . . . . . . 179

$\begin{array}{ll}\text { A Inverse for an overconstrained system } & 180\end{array}$ 


\section{List of Figures}

2-1 General location of SIMI-94 East Camp. . . . . . . . . . . 28

2-2 Seismo-acoustic arrays deployed by MIT/WHOI on and around the SIMI-94 East Camp floe. . . . . . . . . . . . . . . . . 30

2-3 Horizontal surveillance hydrophone array of the SIMI-94 East Camp. 31

2-4 Ice events located by the real-time beamforming on horizontal hydrophone array. . . . . . . . . . . . . . 32

2-5 Directions of the geophone axes. . . . . . . . . . . . 34

2-6 Simple receiver configuration for establishing the relations between arrival times. . . . . . . . . . . . . . 40

2-7 Two examples of the cross-correlation between X-components of the geophones. . . . . . . . . . . . . . . . 41

2-8 Example of the least-square fit to arrival times on 4 geophones. . . . 43

2-9 Locations of events determined by standard least-square fit. . . . . . 44

2-10 Stacked time series of the X-component (North) of the velocity for four events at the beginning of the RLAM tape $26 \ldots \ldots \ldots 47$

2-11 Locations of events time series for which have striking similarities (compare Fig. 2-10). . . . . . . . . . . . . . . .

2-12 Location of the event that produced the vertical velocities characteristic for the flexural wave dispersion pattern. . . . . . . . . . .

2-13 Stacked time series of vertical velocities for the event that produced the vertical velocities characteristic for the flexural wave dispersion pattern. 53

2-14 Results of the processing of the vertical velocity component recorded by the geophone $\# 5$ using multiple filter technique. . . . . . . . . 54 
2-15 Dispersion curves of flexural wave on several geophones. . . . . . 55

2-16 Prograde and retrograde senses of the particle motion. . . . . . . 56

2-17 Hodographs of velocity and displacement in the flexural wave arrival for geophone \#5. . . . . . . . . . . . . 57

2-18 Phase shift between vertical and radial components of motion recorded by the geophone \#5. . . . . . . . . . . . . 58

3-1 Stacked time series of the horizontal $(\mathrm{X})$ component of velocity on 5 geophones which serves as an example of the case when cross-correlation of time series performs poorly. ............... 61

3-2 Plane of polarization of signals considered in MPD processing. . . . . 62

3-3 Two different kinds of polarization of seismo-acoustic signals in the vertical plane: rectilinear and elliptical motion. . . . . . . . . 63

3-4 Schemes of processing in HV/HiV MPD methods. . . . . . . . . 64

3-5 Explanation of two coordinate systems relevant to the geophone data. 67

3-6 Processing results of event \#3 from file \#5 of tape RLAM-26 with the HV MPD method. . . . . . . . . . . . . . . . 68

3-7 Processing results of event \#3 from file \#5 of tape RLAM-26 with the HiV MPD method. . . . . . . . . . . . . . 69

3-8 Geophone array layout. . . . . . . . . . . . . 70

3-9 Processing results of event \#5 from file \#5 of tape RLAM-26 with the HV MPD method. . . . . . . . . . . . . . 71

3-10 Processing results of event \#5 from file \#5 of tape RLAM-26 with the HiV MPD method. . . . . . . . . . . . . 72

3-11 Best least-square fit line for processing results of event \#3 from file \#5 of tape RLAM-26 with the HiV MPD method for geophone \#1. . .

3-12 Best least-square fit line for processing results of event \#3 from file \#5 of tape RLAM-26 with the HiV MPD method for geophone \#2. . .

3-13 Best least-square fit line for processing results of event \#3 from file \#5 of tape RLAM-26 with the HiV MPD method for geophone \#3. . . 
3-14 Best least-square fit line for processing results of event \#3 from file \#5 of tape RLAM-26 with the HiV MPD method for geophone \#4. . .

3-15 Best least-square fit line for processing results of event \#3 from file \#5 of tape RLAM-26 with the HiV MPD method for geophone \#5. . .

3-16 Results of the triangulation of the source location by best least-square fit lines for event \#3 from file \#5 of tape RLAM-26 processed by the HiV MPD method.

3-17 Results of the triangulation of the source location by best least-square fit lines for event \#5 from file \#5 of tape RLAM-26 processed by the HiV MPD method.

3-18 Ranges from array apex versus time for events located by triangulation using HiV MPD processing results. . . . . . . . . . . . .

3-19 Event locations determined by triangulation using HiV MPD processing results overlayed on the aerial photo of the "ice island". . . .

4-1 Satellite synthetic aperture radar images of SIMI-94 site on two dates: April 17 and April 18, 1994. . . . . . . . . . . . . . . 89

4-2 High-resolution satellite synthetic aperture radar image of SIMI-94 site recorded at $9.48 \mathrm{pm}$ GMT on April 18, 1994 . . . . . . . . . 90

4-3 Geophone arrays $\mathbf{A}$ and $\mathbf{B}$ deployed on SW shore of new lead $2 \mathrm{~km}$ NE of camp. . . . . . . . . . . . . . . . . . . . 92

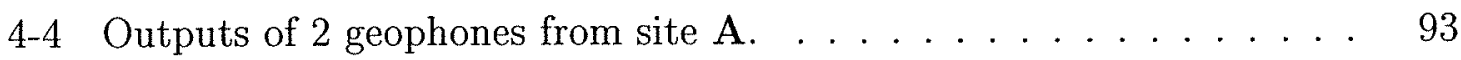

4-5 Autocorrelation for the Y-component of the ice particle velocity recorded by the geophone \#1b from site A. . . . . . . . . . . . .

4-6 Two-dimensional, horizontal wavenumber spectrum for event \#2 (site A, Y-axis outputs of 5 geophones) at $15 \mathrm{~Hz} \ldots \ldots \ldots \ldots 9$

4-7 Directions of wave arrivals for ice events \#2 and \#3. . . . . . 97

4-8 Non-dimensional dispersion curves of the first two fundamental Lamb modes for $c_{p}=3500 \mathrm{~m} / \mathrm{s}, c_{s}=1800 \mathrm{~m} / \mathrm{s} \ldots \ldots \ldots \ldots \ldots$

$4-9$ Edge wave model geometry. . . . . . . . . . . . . . 113 
4-10 Comparison of model dispersion and the phase velocity estimates obtained by conventional beamforming of events \#2 and \#3. . . . . . . 11.8

5-1 Definition of the fault-orientation parameters (strike $\phi_{s}$, dip $\delta$ ) and slip-direction (rake $\lambda$ ) according to [1] . . . . . . . . . 121

5-2 Fault motion with dip angle $\delta$ for 3 different modes of cracking. . . . 121

5-3 Sound profile in the water on April 22, 1994. . . . . . . . . . . 122

5-4 Radiation pattern for a dip-slip crack with a dip angle of $\delta=0^{\circ}$ in the near-field of the source at a frequency of $20 \mathrm{~Hz}$ : vertical component of velocity. . . . . . . . . . . . . . . . .

5-5 Radiation pattern for a dip-slip crack with a dip angle of $\delta=0^{\circ}$ in the near-field of the source at a frequency of $20 \mathrm{~Hz}$ : radial component of

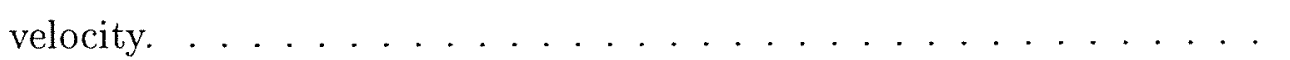

5-6 Radiation pattern for a dip-slip crack with a dip angle of $\delta=0^{\circ}$ in the near-field of the source at a frequency of $20 \mathrm{~Hz}$ : transverse component of velocity. . . . . . . . . . . . . . . . 125

5-7 Radiation pattern for a strike-slip crack with a dip angle of $\delta=0^{\circ}$ in the near-field of the source at a frequency of $20 \mathrm{~Hz}$ : vertical component

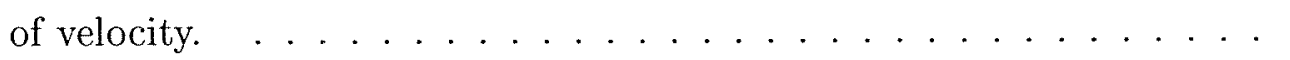

5-8 Radiation pattern for a strike-slip crack with a dip angle of $\delta=0^{\circ}$ in the near-field of the source at a frequency of $20 \mathrm{~Hz}$ : radial component of velocity. . . . . . . . . . . . . . . 126

5-9 Radiation pattern for a strike-slip crack with a dip angle of $\delta=0^{\circ}$ in the near-field of the source at a frequency of $20 \mathrm{~Hz}$ : transverse component of velocity. . . . . . . . . . . . . 127

5-10 Radiation pattern for a strike-slip crack with a dip angle of $\delta=90^{\circ}$ in the near-field of the source at a frequency of $20 \mathrm{~Hz}$ : vertical component of velocity. 
5-11 Radiation pattern for a strike-slip crack with a dip angle of $\delta=90^{\circ}$ in the near-field of the source at a frequency of $20 \mathrm{~Hz}$ : radial component

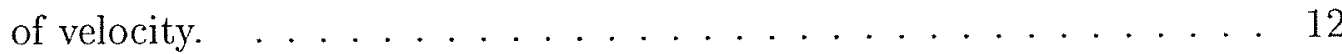

5-12 Radiation pattern for a strike-slip crack with a dip angle of $\delta=90^{\circ}$ in the near-field of the source at a frequency of $20 \mathrm{~Hz}$ : transverse component of velocity.

5-13 Radiation pattern for a tensile crack with a dip angle of $\delta=0^{\circ}$ in the near-field of the source at a frequency of $20 \mathrm{~Hz}$ : vertical component of velocity. . . . . . . . . . . . . . . . . . . . .

5-14 Radiation pattern for a tensile crack with a dip angle of $\delta=90^{\circ}$ in the near-field of the source at a frequency of $20 \mathrm{~Hz}$ : vertical component of

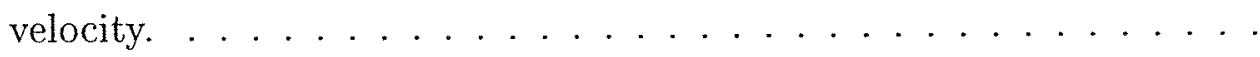

5-15 Radiation pattern for a tensile crack with a dip angle of $\delta=90^{\circ}$ in the near-field of the source at a frequency of $20 \mathrm{~Hz}$ : radial component of velocity

5-16 Radiation pattern for a tensile crack with a dip angle of $\delta=90^{\circ}$ in the near-field of the source at a frequency of $20 \mathrm{~Hz}$ : transverse component of velocity.

5-17 Flow-chart of the analytical radiation pattern fit for each component of motion.

5-18 Locations of five events in Cluster \#1.

5-19 Stacked time series of the horizontal component of velocity for four

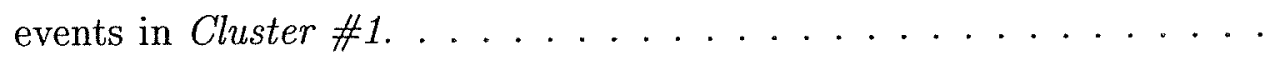

5-20 Best-fit radiation pattern models for event \#5 from Cluster \#1: (a) vertical component of motion; (b) radial component of motion. . . .

5-21 Best-fit radiation pattern models for event \#7 from Cluster \#1: (a) vertical component of motion; (b) radial component of motion. . . . . 138

5-22 Locations of seven events at the beginning of tape RLAM-26. . . . . 140

5-23 Best-fit models of radiation patterns for event \#10: (a) vertical component of motion; (b) radial component of motion. . . . . . . . . . 141 
5-24 Results of fits by two models for the vertical component of motion during event \#10: (a) dipole model; (b) MD-type model. . . . . . . . 141

5-25 Results of fits by two models for the vertical component of motion during event \#16: (a) monopole model; (b) dipole model. . . . . . . . 142

5-26 Results of fits by two models for vertical component of motion during event \#16: (a) quadrupole model; (b) MD-type model. . . . . . . . . 142

5-27 Locations of four events from file \#5 of tape RLAM-26. . . . . . . . . 144

5-28 Best-fit model of radiation pattern for the vertical component of motion during event \#5 from file \#5 of tape RLAM-26. . . . . . . . . . . . 145

5-29 Best-fit models of radiation patterns for event \#5 from file \#5 of tape RLAM-26: (a) radial component of motion; (b) transverse component of motion. . . . . . . . . . . . . . . . 145

5-30 Best-fit models of radiation patterns for event \#11 from file \#5 of tape RLAM-26: (a) radial component of motion; (b) transverse component of motion. . . . . . . . . . . . . . . . 146

5-31 Best-fit models of radiation patterns for event \#12 from file \#5 of tape RLAM-26: (a) vertical component of motion; (b) transverse component of motion. . . . . . . . . . . . . . . 146

5-32 Best-fit models of radiation patterns for event \#9 from file \#5 of tape RLAM-26: (a) radial component of motion; (b) transverse component of motion. . . . . . . . . . . . . . . . . 147

5-33 Standard deviation between experimental data and the quadrupole nodes as a function of strike angle $\varphi_{0}$ and $-L_{\max }$ for the transverse component of motion during event \#9 from file \#5 of tape RLAM-26. 148

5-34 Results of the determination of fault type for several ice events from "ice island" site. . . . . . . . . . . . . . . . . . . . . . 149

5-35 Directions of the fault motion for several events from "ice island" site. 150

6-1 Standard model of the displacement spectrum of an earthquake. . . . 153 
6-2 Vertical displacement spectra of the event \#3 at the beginning of the tape RLAM-26. . . . . . . . . . . . . . . . . . . . 154

6-3 Vertical displacement spectra of event \#5 at the beginning of tape RLAM-26. . . . . . . . . . . . . . . . . 155

6-4 Vertical displacement spectra of event \#9 at the beginning of tape RLAM-26. . . . . . . . . . . . . . . . . . 155

6-5 Vertical displacement spectra of event \#11 at the beginning of tape RLAM-26. . . . . . . . . . . . . . . . . . 156

6-6 Vertical displacement spectra of event \#12 at the beginning of tape RLAM-26. . . . . . . . . . . . . . . . . . 156

6-7 Vertical displacement spectra of event \#13 at the beginning of tape RLAM-26. . . . . . . . . . . . . . . . . . 157

6-8 Two standard models of slip history accepted in seismology: (a) Haskell's model; (b) Brune's model. . . . . . . . . . . . . . . . . . . . . . 159

6-9 Slip history for the horizontal component of motion during event \#1 from file \#1 of tape RLAM-26. . . . . . . . . . . . . 16

6-10 Slip history of two events at about $38 \mathrm{sec}$. from the beginning of the tape RLAM-26. . . . . . . . . . . . . . . . 162

6-11 Slip history of the first event from Fig. 6-10. . . . . . . . . . 163

6-12 Slip history of the second event from Fig. 6-10. . . . . . . . . . 164

6-13 Time series of the vertical component of velocity on the geophone \#2 at the beginning of tape RLAM-26. . . . . . . . . . 166

6-14 Autocorrelation function for the time series of the vertical component of velocity on geophone \#2 at the beginning of tape RLAM-26 (see Fig. 6-13).

6-15 Slip function for part of the time series shown on Fig. 6-13 exhibiting stick-slip type of behavior. . . . . . . . . . . . 168

6-16 Spectrogram of vertical velocity on geophone \#2 at the beginning of tape RLAM-26. . . . . . . . . . . . . . . . . . . . . 169

6-17 Stress drop definition according to $[21] \ldots \ldots \ldots \ldots \ldots \ldots$ 


\section{List of Tables}

4.1 Ice events timing intervals . . . . . . . . . . . . . 91

5.1 Correspondence between analytical models and crack types. . . . . 132

6.1 Event parameters for several events at the beginning of tape RLAM-26.174 


\section{Chapter 1}

\section{Introduction}

\subsection{Motivation}

For several years there has been a great interest in arctic ice mechanics research even after the arctic region lost its strategic importance for military applications. One of the reasons is that the ice cover can serve as a sensitive indicator of changes in global climate (e.g. the greenhouse effect). Another reason is that the Arctic contains rich mineral and oil resources and in order for mining facilities to be able to withstand harsh environmental conditions, we need to have a better understanding of the processes of the sea ice mechanics. This knowledge can also help in improving navigation conditions for ships in arctic seas.

\subsection{Objectives}

An example of this interest is the Sea Ice Mechanics Initiative (SIMI) experiment carried out in a joint effort by several institutions in Spring of 1994. The objective of SIMI was to obtain basic physical understanding of the highly non-linear process by which environmental forcing (including atmospheric conditions, oceanic currents, heat flux etc.) leads to formation of the dramatic features characterizing the macroscopic Arctic ice, according to ([52],[45]). This objective required a method of monitoring the mechanical processes in ice that could determine the development of stress and strain 
distributions in space as well as time, providing both coverage and resolution. This requirement suggested acoustic and seismo-acoustic remote sensing as an attractive and feasible option. One such remote sensing method is based on the detection and analysis of the stress waves generated in the ice and the water column by fracture formation in the ice cover. When a crack is formed, the stresses are released over the crack surface, producing stress waves radiated in a pattern characteristic for that particular fracture process. The parameters describing the fracture process can therefore be determined by inversion of the radiated stress wave field.

The traditional experimental setup for remote sensing of ice events used the horizontal hydrophone array to monitor the fracture development in the far-field. This approach does not provide sufficient spatial resolution to invert for all fracture parameters describing the ice mechanical development. To achieve this the sensors need to be deployed in the vicinity, or near-field, of the ice fracture events.

Recent experiments ([33]) have demonstrated a resolution superiority of threecomponent geophones compared to hydrophones for near-field observation of seismoacoustic propagation in the ice sheet. Additionally, the geophones allow direct meatsurement of the characteristics of the wave propagation in the ice, whereas hydrophones give information only about the part of the event energy which was transferred to acoustic waves in the water due to the interaction at the ice-water interface. As a result, the ice crack characteristics appear in hydrophone data only as second order effects. Also, the vector nature of the data recorded by three-component geophones provides substantially richer information than hydrophones about the chas:acteristics of the waves in the ice sheet.

In the following I will analyze and model the geophone data collected by the joint MIT/WHOI group during SIMI-94 experiment in order to address some of the objectives of Sea Ice Mechanics Initiative. The specific objectives of this analysis will be identification of ice events and estimation of their important parameters from the elastic waves generated by the events in the ice cover. 


\subsection{Previous work}

This review of the previous work is organized along the following lines: first, I will discuss two directions in the study of the Arctic ambient noise, then I will review literature devoted to the application of earthquake seismology methods to the analysis of ice event data.

The study of the Arctic ambient noise usually follows two distinct directions. One direction is to correlate different characteristics of ambient noise to environmental parameters such as heat flux, temperature and wind. The other direction is to study individual ice events which form the average ambient noise in Arctic. In that approach the implicit assumption is that the ambient noise in Arctic is dominated by the mechanical processes in the ice cover [8].

The first direction started with work by Milne and Ganton [35]. They identified the major source of ambient noise for shore-fast ice during Spring and Winter as thermal tensile cracks, while during Summer - as relative motion of ice floes. In their next work [12] for Winter season they identified two different spectral regions for ambient noise: lower frequencies (between 200 and $800 \mathrm{~Hz}$ ) showed an impulsive character of radiation with noise level declining rapidly with increase of temperature; the other frequency range (between $1 \mathrm{kHz}$ and $10 \mathrm{kHz}$ ) had Gaussian character and was attributed to wind forcing due to dependence on wind speed.

Makris and Dyer [29] showed that long-term variations of low-frequency ambient noise under pack ice of the central Arctic Ocean correlated highly with composite measures of stress applied to the ice by wind, current, and drift. These composites were identified as the horizontal ice stress and the stress moment, and were derived from meteorological and oceanographic data observed simultaneously with the noise. They also concluded that atmospheric cooling (a known high correlate of mid-frequency noise under the ice) was not important at low frequencies. In contrast to the results for the central Arctic, they showed [30] that in the marginal ice zone ice stress, ice moment, and wind stress magnitude were poor correlates for the lowfrequency ambient noise. They concluded that for that Arctic region the primary 
correlate of the noise was surface gravity wave forcing, with the most likely mechanisms for sound generation being the flexural floe failure and unloading motion, within a few kilometers of the ice edge.

The study of event source mechanisms was started by Milne. He considered thermal tensile cracks as the noise source mechanism [34] and found that the ambient noise level in the 150-300 Hz band correlated well with the changes in air temperature. In [36] he suggested that the dominating mechanisms of ambient noise generation may vary with the Arctic region. This assumption was later confirmed in other experiments. Lewis et al. [26],[27] corrected some of the conclusions of the theory for thermal cracking in ice by Milne et al. They showed that heat flux, rather than air temperature, was the main factor in ice fracturing under thermal tension [26]. Their other main contribution to the study of event mechanisms was the calculation of the ice stress due to the thermal effect [27].

Pritchard [40] suggested that the energy dissipated during the ridging process was the proper measure of the ambient noise source level. Numerical simulations showed that a significant amount of energy and ambient noise could be explained that way. The model was also physically attractive and properly explained lack of noise when winds were high but the ice was strong enough to resist ridging.

The theoretical modeling of ice cracking events as a source of acoustic energy radiated into the water was started by Stein [50]. He used a monopole source in the ice plate to model the tensile ice cracking. His was an ad hoc radiation model appropriate only for events whose openings were small compared to ice thickness. For more complex events a monopole source should be combined with a quadrupole source as suggested by Langley [23], [24], [25]. Kim [22] used a computational approach to the crack radiation problem. He developed an analytical and numerical model of the elastic wave field in range independent elastic environments for various seismic source mechanisms including shear and tensile cracks. Using compact source representation with fault surface in arbitrary direction, he derived the basic Green's function solution for propagation in stratified elastic media which was incorporated into a numerical model. Kim extended that solution to include more complete cracking mechanisms, 
like non-compact and moving cracks. He also studied some effects of anisotropy on the acoustic emission.

Dyer [7], [8] discussed several possible event mechanisms of noise generation in the low- and mid-frequency ranges of the noise spectrum with the ridge unloading model among them. He also introduced a slip model for ice fracture. Chen [4] used these models in her study of ice event mechanisms in the Marginal Ice Zone. The application of similar ideas to the central Arctic region was carried out by Stamoulis in her thesis [49]. She also introduced event identification by their radiation patterns into the study of events mechanisms.

The above studies were mostly concerned with the sound radiated into the water by ice events. The more direct way to study ice events mechanisms is to investigate the elastic field produced by the event in the ice sheet itself. This could be achieved by using geophones, sensors that are coupled directly to the ice by freezing, and capable of measuring the particle velocity of ice motion directly. One of the first studies of ice events in the Arctic using geophones was conducted by Hunkins in 1960 [18]. He successfully managed to isolate the flexural waves in ice by investigating the sense of particle orbital motion in the wave near the ice surface. For this purpose he plotted the output of the vertical axis of the sensor vs the output of one of the horizontal axes. His diagrams could not show the true particle motion due to frequency dependence of the phase shift. His results showed the elongated horizontal ellipse with retrograde direction of motion just like it was predicted by Sato [42],[43]. According to Hunkins, only flexural waves gave the clear picture of the particle orbital motion, other waves just produced a jumble on the hodographs, whereas later [33] longitudinal waves have been also identified. In some situations this method could help to distinguish different wave types by their planes of polarization. Note that the above hodograph method could only be applied to data from multi-axis sensors such as three-component geophones.

Relatively recently, Yang and Giellis used an array of geophones for the determination of dispersion characteristics of flexural wave in ice generated by striking sledge hammers against a steel pipe frozen into the ice during their Arctic experiment in 
1988 [54], [55]. However, they used only the vertical component of velocity recorded by the geophones in the data processing. The data processing scheme made use of the Choi-Williams display [5] for selected vertical-axis geophones in which the signal intensity was plotted as a function of time and frequency. Then those displays were converted into group-velocity versus frequency plots using the known receiver range and measured travel time. Finally, converted plots (associated with nine vertical-axis geophones at different ranges) were summed in intensity (incoherent processing).

B.E. Miller ([33], [32]) used geophone data to precisely locate ice events in the PRUDEX-87 experiment. He found that the geophones give much more precise estimates of locations for experimental shots than hydrophones do. He also used that data to estimate the location of an ice ridge. In the geophone time series generated by under-ice detonations, he found not only the expected longitudinal and flexural waves in the ice plate, but also unexpected horizontally polarized transverse (SH) waves. The need to determine the origin of the $\mathrm{SH}$ wave from the received time series additionally highlighted the dramatic superiority of geophones over hydrophones in this application. Miller also conducted the inversion of the geophone data for the ice sheet's low-frequency elastic parameters initially by modeling the ice as a single homogeneous isotropic plate using SAFARI. After that a modified stationary phase approach was used to extend SAFARI modeling to invert for the parameters of two ice half-plates simultaneously.

The stress relief in arctic ice have features similar to that of the stress relief in the earth's crust. Hence, earthquake mechanics should provide a useful starting point for examining seismo-acoustic signals radiated by the ice crack [10]. By far, the most common types of cracks in ice mechanics are tensile cracks, dip-slip and strike-slip (both with dip angle $\delta \approx 0^{0}[22]$ ). For these kinds of cracks the general model was adapted by D. Farmer and Y. Xie in [10]. By analogy with results of theoretical models of propagating earthquake cracks, they assumed that the source moves with the developing crack. The authors adapted a concept introduced in seismology by Haskell and modeled the source as a sinusoidally roughened ramp function, thereby inducing a significant fraction of the energy in a primary or base-band lobe (200-300 
$\mathrm{Hz}$ ). The frequency of this lobe is governed by the propagation speed and by the coherent length of a crack segment. The high-frequency peak $(\approx 5 \mathrm{kHz})$ in this model is related to the vertical length scale of the crack. In this approach the calculation of event parameters requires an assumption regarding the rupture velocity $v_{r}$. Using [31] one gets

$$
v_{r}(\max ) \approx 0.63 \beta
$$

where $\beta$ is the shear wave speed. After that the crack length $L$ is obtained from the base-band frequency $f_{b}$ as

$$
L=\frac{v_{r}}{2 f_{b}}
$$

This actually gives the length of the coherent crack segment (segment on which crack can be treated as a fault propagation having constant speed).

The other similarity of seismology to seismo-acoustics is the use of similar sensors. Both fields use devices that measure particle velocity of the medium quite extensively, though, unlike seismo-acoustics, in seismology an array of other instruments is also deployed (among them accelerometers and devices that measure particle displacement directly). However, until recently (about mid-80s) seismologists did not really use arrays of three-component sensors. They limited their attention to arrays of one-component sensors or single three-component sensors. To my knowledge, the paper [19] by A. Jurkevics pioneered the use of the array of three-component sensors in seismology. The analysis technique in this research was based on a time-domain algorithm originally proposed by Flinn in 1965. In this algorithm the polarization properties of the seismic waves were computed within sliding time windows by solving the eigenproblem for the covariance matrix for three components of motion of each geophone separately. This technique was extended to multiple three-component sensors in an array configuration by averaging covariance matrices for the different sensors. In this case a $1 / \mathrm{M}$ reduction in the estimation variance was obtained ( $\mathrm{M}$ is the number of sensors), when the noise and local scattering effects are uncorrelated. 


\subsection{Thesis contributions}

- Determination of polarization characteristics of the elastic waves using multicomponent geophone data.

Polarization methods are well known in seismology, but they never have been used for ice event data processing. In this work one of the polarization methodis (so called Motion Product Detector method) has been successfully applied for localization of ice events and determination of polarization characteristics of the elastic waves generated by these events. This application demonstrated the feasibility of using the polarization method for ice event data processing, because it allows one to identify areas of high stress concentration and "hot spots" in ridge building process.

Furthermore, the polarization characteristics of elastic waves could be used for establishing the physics of the processes in the ice sheet which lead to generation of ice events.

- The discovery of the new phenomenon: "edge waves", which are waves propagating back and forth along a newly opened ice lead.

These waves exhibit a quasi-periodic behavior suggesting some kind of stick-slip generation mechanism somewhere along the length of the lead. The propagation characteristics of these waves were determined using seismic wavenumber estimation techniques. In the low-frequency limit the dispersion can be modeled approximately by an interaction at the lead edges of the lowest order, antisymmetric modes of the infinite plate.

- Identification of source mechanisms for events based on their radiation patterns. This identification was carried out using near-field seismo-acoustic emission of natural ice events. As far as I know, this has never been done before directly. Previous work on natural ice event identification was done indirectly: using acoustic energy radiated into the water through coupling of into water column of elastic energy in the ice sheet radiated by ice events. 
- Estimation of parameters of fault processes in Arctic ice.

Stress drop, seismic moment and the type of ice fracture were determined using direct near-field measurements of seismo-acoustic signals generated by ice events. Obtained values of fracture parameters were in good agreement with those obtained by C.F. Chen [4] for marginal ice zone.

\subsection{Thesis organization}

First, in Chapter 2 I give a short overview of the SIMI-94 experiment, data description and initial stages of data processing: preprocessing, event detection and localization using traditional least-square fit to interarrival times. At the end of this chapter I show some results for flexural wave arrival. In Chapter 3, the polarization processing of the geophone data is described. This method allows one to take advantage of the multi-component nature of the geophone data. Chapter 4 is devoted to the new phenomenon found in the geophone data collected in the vicinity of an ice lead, the so-called "edge waves": waves propagating back and forth along an ice lead. In Chapter 5 I describe the results of event source mechanisms identification based on radiation patterns. Chapter 6 is devoted to event parameter estimation. The major parameters estimated are the seismic moment and stress drop in the fault. Finally, in Chapter 7, I present a summary of the thesis and suggestions for future work. 


\section{Chapter 2}

\section{Sea Ice Mechanics Initiative \\ (SIMI) Experiment overview and data preparation procedure}

\subsection{Overview of the SIMI experiment}

As part of the Office of Naval Research (ONR) Sea Ice Mechanics Initiative (SIMI), a real-time monitoring and processing program for acoustic emission from ice fracture and ridge-building events was established. A wide aperture horizontal hydrophone array was used in combination with a vertical line array to record the acoustic signals, which were then passed through a focused beamformer for real-time generation of ice seismicity maps. A number of rapidly deployable geophone arrays were used in active zones to measure the acoustic emissions in the near field for detailed seismic event analysis.

The primary objective of the ONR Sea Ice Mechanics Initiative was to develop a fundamental understanding of the mechanical behavior of sea ice undergoing environmental forcing due to currents, wind and heat flux. The several Arctic acoustics experiments of the past have made it clear that ice fracturing is the dominant source of ambient noise, in turn suggesting that this phenomenon forms the major compo- 


\section{SIMI-94 Camp Location}

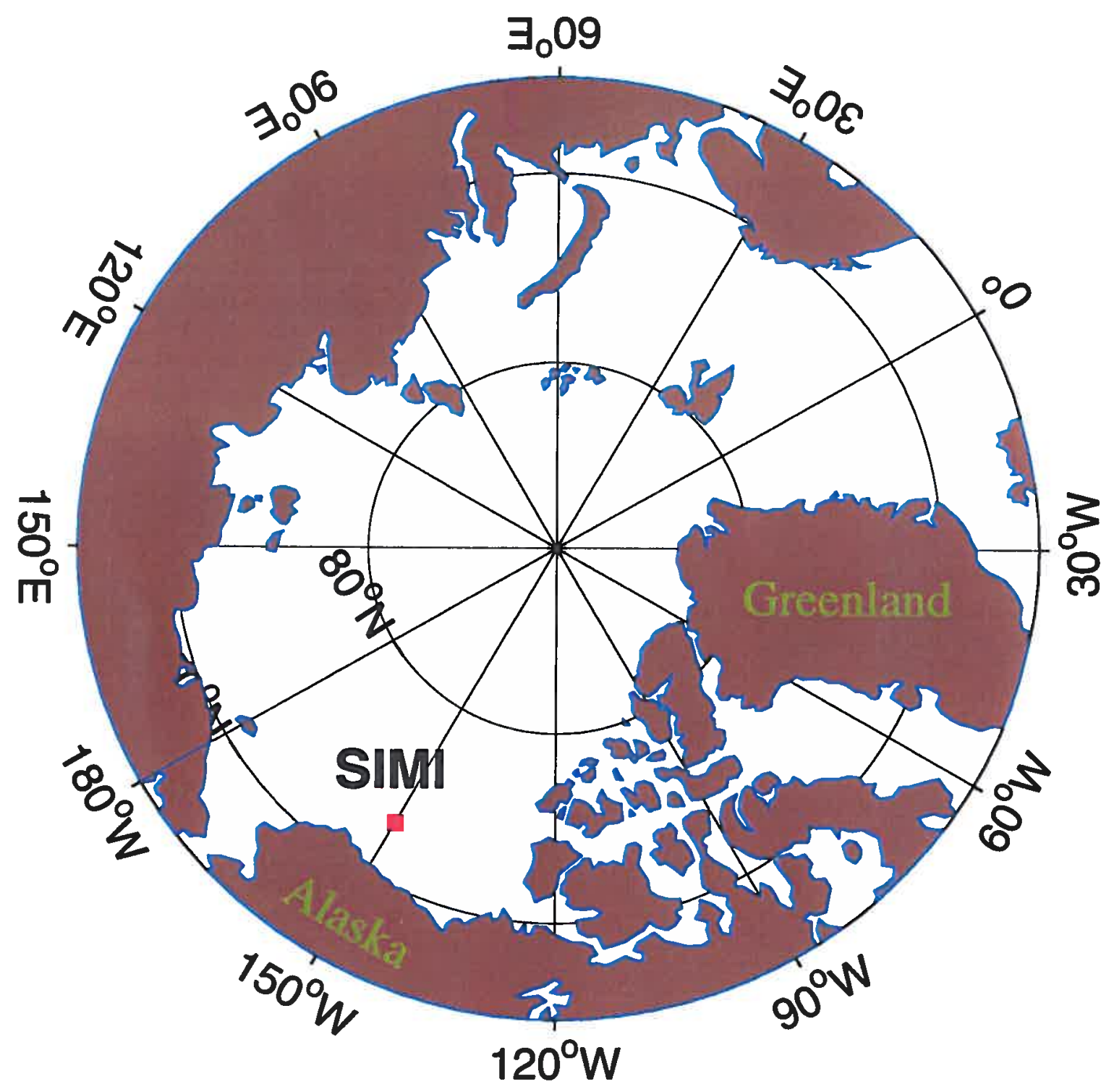

Figure 2-1: General location of SIMI-94 East Camp. The GPS coordinates of the camp on April 22, 1994 were $73^{\circ} 00^{\prime} 56^{\prime \prime} N$ and $149^{\circ} 53^{\prime} 55^{\prime \prime} W$. 
nent of the physical processes leading from environmental forcing to the development of macroscopic ice features such as ridges, leads and raftings. On this background, the specific objective of the MIT/WHOI SIMI effort was to develop a basic understanding of these ice fracturing processes and their relation to the environmental forcing.

The main experiment of the SIMI effort was conducted in the Spring of 1994 approximately $400 \mathrm{~km}$ north of Prudhoe Bay, Alaska (see Fig. 2-1). The GPS coordinates of the camp on April 22, 1994 were $73^{\circ} 00^{\prime} 56^{\prime \prime} \mathrm{N}$ and $149^{\circ} 53^{\prime} 55^{\prime \prime} \mathrm{W}$.

\subsection{SIMI arrays and systems}

\subsubsection{Surveillance Hydrophone Array}

As a mechanism for monitoring and localizing ice activity during the Spring SIMI field experiment, MIT/WHOI deployed two hydrophone arrays, comprising a seismicity 'surveillance' capability approximately $300 \mathrm{~m}$ from the main camp area. A vertical line array (VLA) of hydrophones, having 32 sensors linearly spaced from a depth 62 to 279 meters was deployed at the apex of the site (the origin of the coordinate system on the plot) shown in Fig. 2-2. Surrounding this APEX was a horizontal array consisting of a 12 hydrophone circular deployment augmented by 12 additional sensors on nominal north-south and east-west legs $240 \mathrm{~m}$ in length (for details see Fig. 2-3). Later, 8 more hydrophones were deployed for a total of 32 in the horizontal array (HA), all at a depth of $60 \mathrm{~m}$. The labels near some hydrophones on Fig. 2-3 and 2-2 indicate the nominal direction and range in meters of the sensors as surveyed relative to the apex of the site. Two USRD J9 acoustic sources ${ }^{1}$ were deployed and each driven with a dual tone signal for use in localizing the VLA channels.

A subset of the recorded data was processed in real time to continuously update seismicity displays. The data were passed through a real-time, focused beamformer,

\footnotetext{
${ }^{1}$ USRD stands for "Underwater Sound Reference Detachment", an organization that is part of the Naval Research Lab. USRD owns various pieces of acoustic equipment that they can lend or rent to other institutions for temporary use. These J9 sources were rented from them by the MIT/WHOI group for the SIMI-94 experiment.
} 


\section{Arrays and Sources}

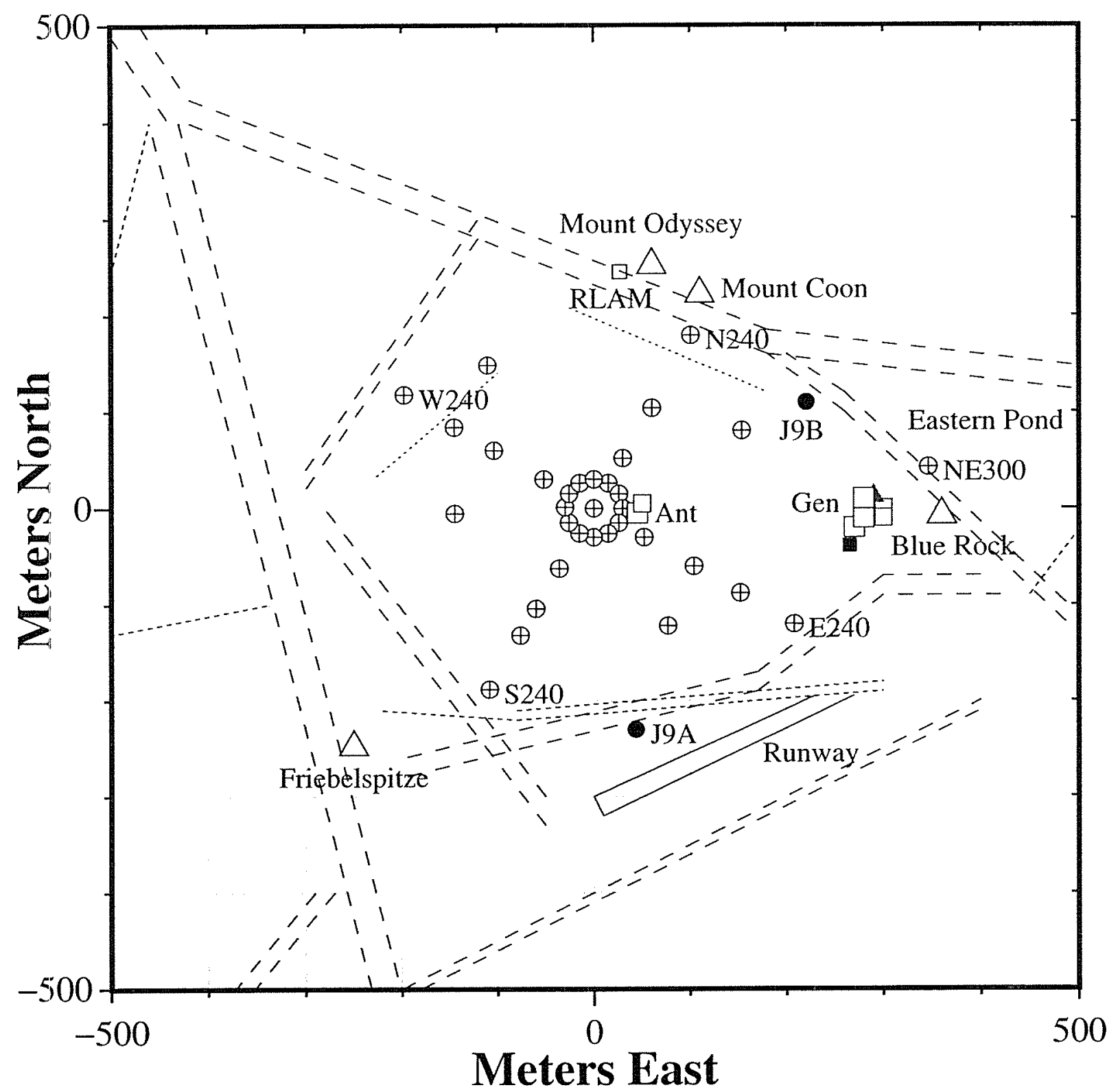

Figure 2-2: Seismo-acoustic arrays deployed by MIT/WHOI on and around the SIMI94 East Camp floe. A total of 32 hydrophones (shown on the figure by the circles with crosses) were deployed in the horizontal array in a combined circular/cross configuration. The labels near some hydrophones nominally indicate the direction and range in meters of the sensors as surveyed relative to the array apex (the origin of the coordinate system on the plot). Only the names of the outermost hydrophones are shown on this figure to avoid cluttering it up. A 32 element vertical array was deployed at the horizontal array apex. Two J-9 acoustic sources (J9A and J9B) were deployed for sensor tracking. Markers with labels Ant and Gen show the locations of the antenna hut and generator hut, respectively. The location of the geophone array near ice mountain called Mount Odyssey is shown by square marker with label RLAM near it. The locations of the ice mountains are shown by triangle markers. 


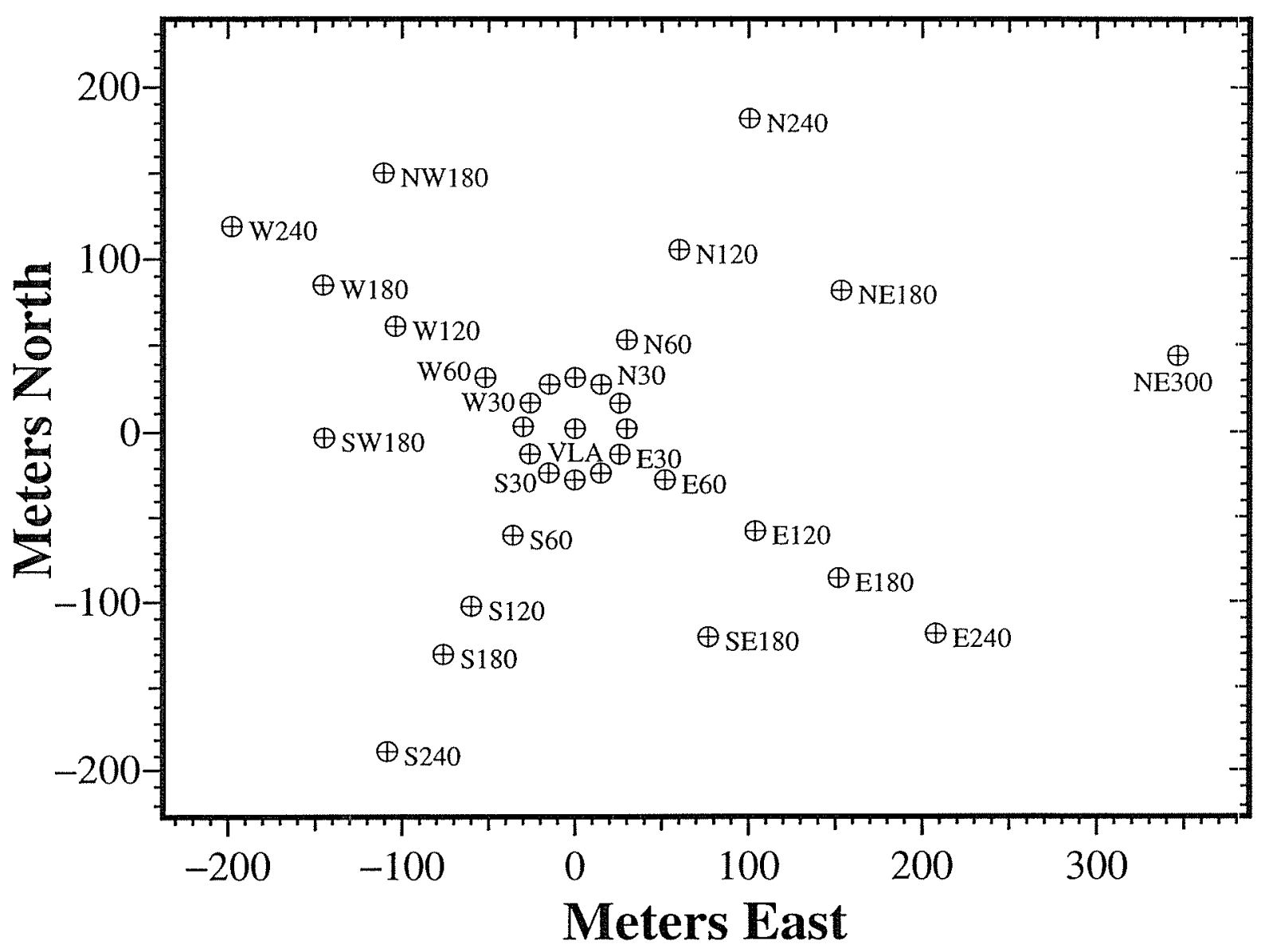

Figure 2-3: Horizontal surveillance hydrophone array of the SIMI-94 East Camp. The hydrophone locations are shown on the figure by the circles with crosses. The labels near some hydrophones indicate the nominal direction and range in meters of the sensors as surveyed relative to the array apex (the origin of the coordinate system on the plot). A 32 element vertical array was deployed at the horizontal array apex (the label VLA reflects this fact). 


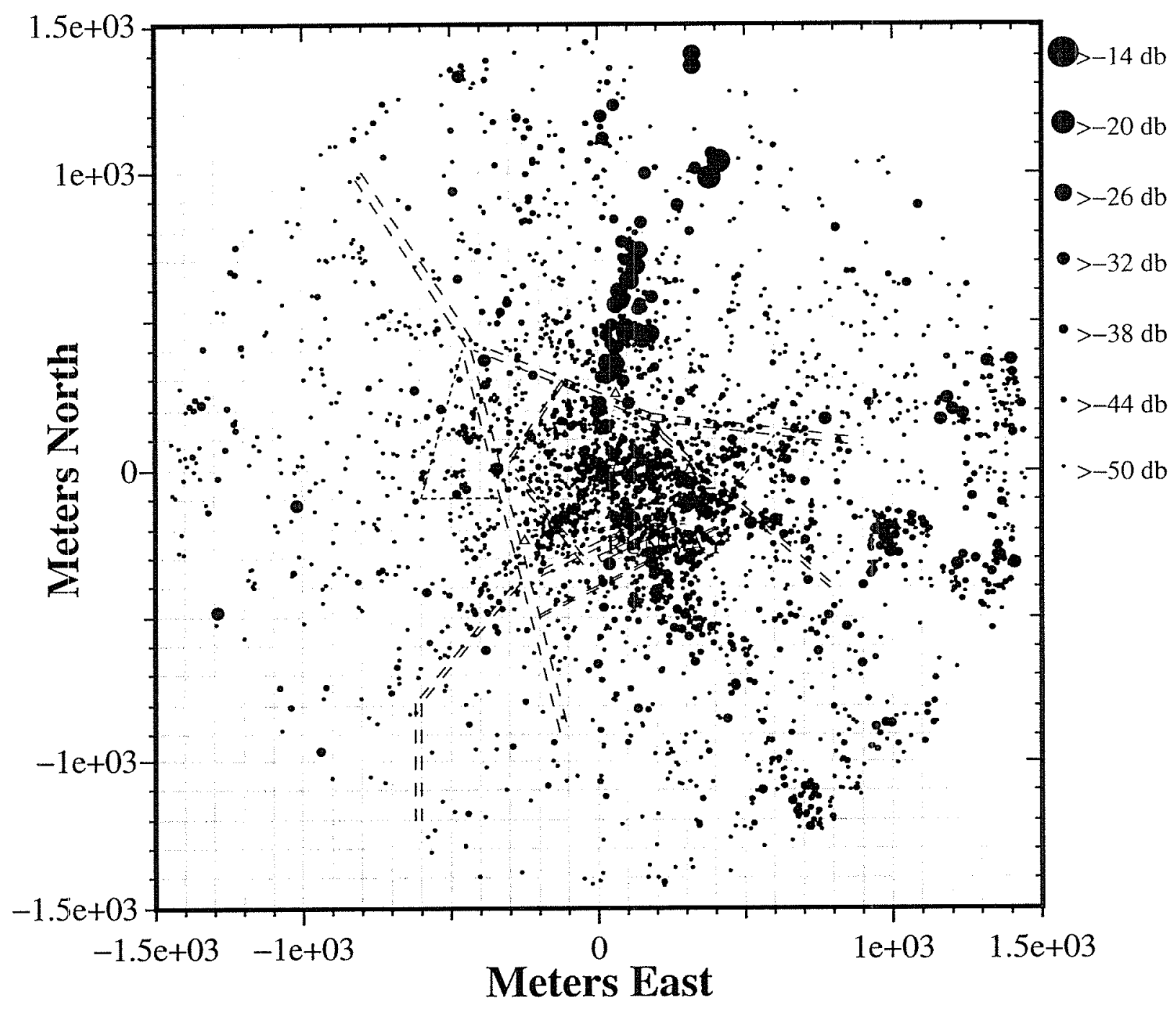

Figure 2-4: Ice events located by the real-time beamforming on horizontal hydrophone array between 098:23.00Z and 098:23.59Z, including major events associated with existing crack immediately north of the new shear ridge (Mount Odyssey on Fig. 22). Such real-time beamforming was used during the experiment to identify the active areas on ice. 
developed specifically for ice event detection and localization by Edward K. Scheer, and hourly event maps were generated. As a characteristic example, Fig. 2-4 shows events detected between 098:23.00Z and 098:23.59Z, including major events associated with an existing crack immediately $\mathrm{N}$ of a new shear ridge (Mount Odyssey). The size of the markers indicate the estimated source levels of the individual events. As a result of this detection, one of the Radio LAN Acquisition Module (RLAM) geophone clusters was deployed in the vicinity of the crack and recorded the continued seismicity for several days following. This example is introduced only to show how active areas were identified during experiment and is not a part of the thesis research.

These results were used to select sites for deploying autonomous RLAMs that acquired geophone data in the near-field of apparently active sites. This data is the primary focus of this thesis.

\subsubsection{Geophone Clusters}

Ice motion, either as a result of natural activity or induced events, was measured directly by portable RLAM systems, each equipped with 5 geophones and 1 hydrophone. The geophones were standard Teledyne units mounted in an $\mathrm{OBS}^{2}$ housing. The low frequency cutoff of each geophone was $4.5 \mathrm{~Hz}$ and its high frequency cutoff was above $10 \mathrm{kHz}^{3}$. At the low frequency cutoff the geophones were damped such that at $4.5 \mathrm{~Hz}$ the amplitude response was down by $3 \mathrm{~dB}$ and continued to roll off as frequency decreased by $6 \mathrm{~dB} /$ octave. Each geophone was actually a three-component cluster which measured three orthogonal components of particle velocity of the ice motion. The geophones were mounted directly on the hard ice using slush, which immediately froze the units to the ice. In case of snow cover present, the ice was first cleared. The typical snow cover was around $30 \mathrm{~cm}$.

These systems were deployed at 8 sites in the vicinity of the SIMI-94 East Camp for varying times throughout the experiment. Data was telemetered via a radio local

\footnotetext{
${ }^{2}$ OBS stands for Ocean Bottom Seismometer

${ }^{3}$ The precise value of the high frequency cutoff is not important, because the maximum sampling frequency for the geophone arrays, as far as I know, was around $4 \mathrm{kHz}$.
} 


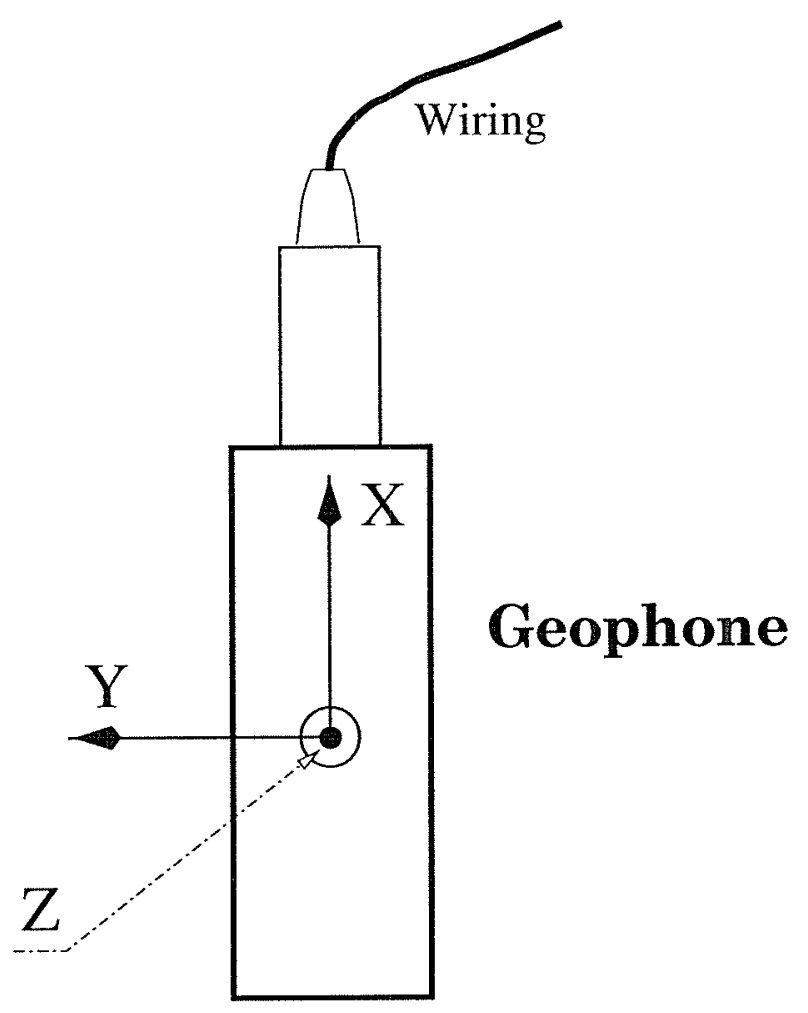

Figure 2-5: Directions of the geophone axes. $X$ axis is oriented along the main axis of the geophone. Both $X$ and $Y$ axes lie in the horizontal plane. $Z$-axis is the vertical axis with positive direction upwards.

area network to a receiving system located at the MIT/WHOI science hut at the SIMI-94 East camp where it was stored on $8 \mathrm{~mm}$ tape in a format similar to the 'surveillance' system. The data bandwidth varied from 400 to $1600 \mathrm{~Hz}$ depending on whether 1, 2 or 3 RLAM units were operating simultaneously.

Data was acquired during selected times from 7 April through 18 April depending on the schedules of other experiments, such as induced fracturing and ice block drops as well as the detection of natural events. During the remainder of the experiment through 23 April, 3 RLAM units were kept in continuous operation near shear ridge 'Mt. Odyssey', the remote $\mathrm{APL} / \mathrm{UW}^{4}$ site, the large lead to the north of the camp (so called "ice river" site), and on a small floe involved in active ridge building approximately $4 \mathrm{~km}$ east of the camp (so called "ice island" site).

\footnotetext{
${ }^{4}$ APL/UW stands for Applied Physics Laboratory of University of Washington
} 


\subsection{Preparation of the SIMI data}

In this section the preparation of the SIMI geophone data is discussed. First, the preprocessing of the data needed to obtain absolute levels of the velocity in ice is discussed. After that, the procedure for event detection is presented. Then follows the description of the standard procedure for event localization. The method begins with the estimation of time delays between different sensors, followed by the procedure for a least-square fit to the interarrival data assuming an unknown propagation speed (the section in text labelled "Search in Cartesian coordinate space"). In this procedure, all possible locations for the source are tested and the choice is made based on the least deviation between calculated and experimental interarrival times. Sometimes, it is advantageous to use different procedure when for each trial propagation speed (as opposed to trial source location in previous method) the standard deviation between calculated and experimental interarrival times is determined and the value of propagation speed (and corresponding source location) which minimizes this difference is chosen. The description of that method (called "Search in propagation speed space") finishes this section.

\subsubsection{Preprocessing of geophone data}

To determine the actual geophone positions, the records of the distances and angles between geophones were used. These records were processed using the program SURVEY, written by Prof. H. Schmidt in FORTRAN programming language. This program used a simulated annealing method to determine the locations of the geophones.

Every attempt was made to orient the main axis of each geophone (axis $X$ on Fig. 2-5) along the direction to magnetic North. The small deviations in direction from magnetic North at each geophone and magnetic deviation at the experimental site were accounted for using conceptual rotation of horizontal components of velocity. So if the original (recorded) horizontal components on the geophone \#i were $X_{\mathrm{rec}}^{i}$ and $Y_{\text {rec }}^{i}$ and rotated $-X^{i}$ (true North component) and $Y^{i}$ (true West component), then the conceptual rotation can be expressed as 


$$
\begin{aligned}
& X^{i}=X_{\mathrm{rec}}^{i} \cos \left(\alpha_{\mathrm{mag}}+\alpha_{i}\right)-Y_{\mathrm{rec}}^{i} \sin \left(\alpha_{\mathrm{mag}}+\alpha_{i}\right) \\
& Y^{i}=X_{\mathrm{rec}}^{i} \sin \left(\alpha_{\mathrm{mag}}+\alpha_{i}\right)+Y_{\mathrm{rec}}^{i} \cos \left(\alpha_{\mathrm{mag}}+\alpha_{i}\right),
\end{aligned}
$$

where: $\alpha_{i}$ - deviation of main axis of the geophone $\# i$ from magnetic North; $\alpha_{\text {mag }}$ magnetic deviation at the experimental site.

The conversion of the raw data to true units $(\mathrm{m} / \mathrm{sec})$ on the magnetic tape involves three steps. First, the RLAMIZE routine written in C-language by Edward K. Scheer converts the raw data value to volts at the input of the RLAM analog-to-digital converters but before fixed gain was applied:

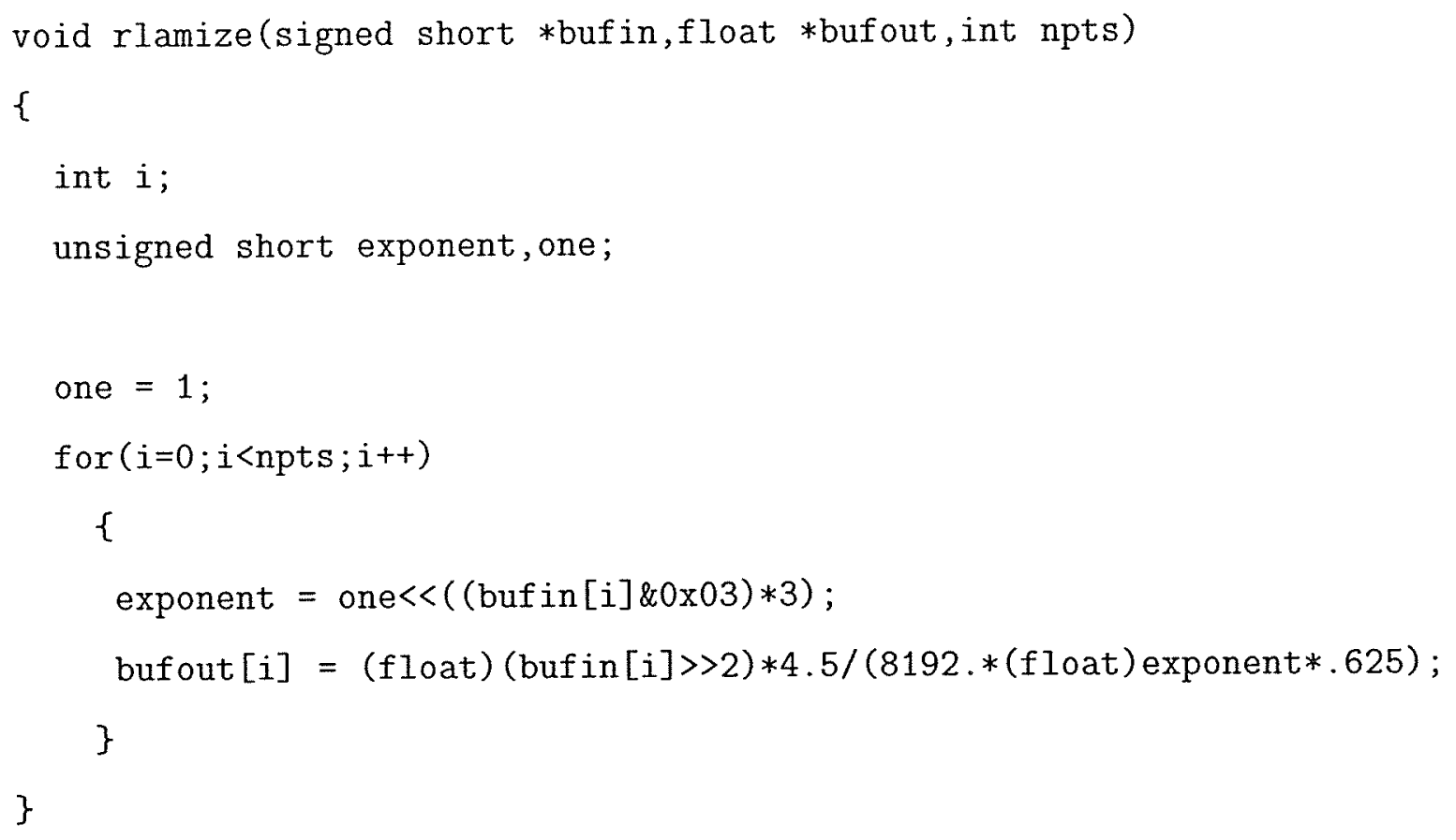

This routine has an input in bufin and output in bufout and it processes npts of 2-byte long data values. To correct the data for a fixed gain, the output of this routine is divided by factor of 100 . The last step in obtaining values in true units is using the geophone sensitivity, which was $642 \mathrm{mV} / \mathrm{in} / \mathrm{sec}$, or $25.3 \mathrm{~V} / \mathrm{m} / \mathrm{sec}$ to convert volts to $\mathrm{m} / \mathrm{sec}$. 


\subsubsection{Event detection procedure}

The first step in the detection algorithm is to read the selected block of data $B$ (say $20 \mathrm{sec}$. long) from the input data file or directly from the magnetic tape. Then the long-time average (LTA) rms energy is computed for each geophone and for each component of the velocity. As a result, we obtain 3 sets of $N$ values $L_{x i}, L_{y i}, L_{x i}$, where index $i$ is a geophone number $(i=1, \ldots, N)$. For example,

$$
L_{x i}=\sqrt{\frac{1}{M} \sum_{j=1}^{M}\left[v_{x i}\left(t_{0}+(j-1) \mathrm{d} t\right)-\mathbb{M}_{x i}\right]^{2}}
$$

where:

$t_{0}$ - the starting time of block $\mathrm{B}$,

$M$ - the number of samples in the block,

$\mathrm{d} t$ - the sampling interval of the recording,

$v_{x i}\left(t_{1}\right)$ - the value of $x$-component of the velocity of the ice particle recorded by the $i$-th geophone at the time moment $t_{1}$,

$\mathbb{M}_{x i}$ - the mean of the $x$-component of velocity recorded by $i$-th geophone over the whole block $B$.

$\mathbb{M}_{x i}$ is defined as

$$
\mathbb{M}_{x i}=\frac{1}{M} \sum_{j=1}^{M} v_{x i}\left(t_{0}+(j-1) \mathrm{d} t\right)
$$

Single-channel LTAs are needed to determine the Signal-to-Noise Ratio (further referred to as SNR). Note that this allows adaptation to varying noise levels in time as we move through the data set by reading a new block from the file or directly from the tape.

Averaging LTAs also across the geophones. we obtain now 3 values: $\mathbb{L}_{x}, \mathbb{I}_{y}$, 
$\mathbb{L}_{z}$. So, for example,

$$
\mathbb{L}_{z}=\frac{1}{N} \sum_{i=1}^{N} L_{z i}
$$

where $N$ is the number of the geophones.

In addition, we keep track of long-time means averaged across the geophones $\mathbb{M}_{x}, \mathbb{M}_{y}, \mathbb{M}_{z}$. For example,

$$
\mathbb{M}_{y}=\frac{1}{N} \sum_{i=1}^{N} \frac{1}{M} \sum_{j=1}^{M} v_{y i}\left(t_{0}+(j-1) \mathrm{d} t\right)
$$

where $t_{0}$ - the starting time of block B, $M$ - the number of samples in the block, $\mathrm{d} t-$ the sampling interval of the recording, and $v_{x i}\left(t_{1}\right)$ - the value of $x$-component of the velocity of the ice particle recorded by the $i$-th geophone at the time moment $t_{1}$.

We choose a window length of analysis $W$ such that it is long enough to encompass the typical signal lengths expected in the recorded time series. The other requirement for the length of window is that it should allow a sufficient time for the signal to appear on all the channels in the analysis window.

Next, the short-time average (STA) rms energy is computed for each geophone and each component of the velocity. As a result we obtain 3 sets of $N$ values $S_{x i}, S_{y i}$, $S_{x i}$, where index $i$ is a geophone number $(i=1, \ldots, N)$.

Using per-channel STAs and averaged over the channels LTAs $\left(\mathbb{L}_{x}, \mathbb{L}_{y}, \mathbb{L}_{z}\right)$ we determine whether the particular channel (particular component of the particular geophone) is "noisy" or not. For example, let us consider the $x$-component of velocity recorded by the $i$-th geophone. Then, if

$$
\left(\mathbb{M}_{x}-2 \mathbb{L}_{x}\right)<S_{x i}<\left(\mathbb{M}_{x}+2 \mathbb{L}_{x}\right)
$$

this particular channel is declared "non-noisy" and can be used for detection purposes in window $W$. Otherwise, it is discarded for detection in this time window.

Next, we determine whether the data within the analysis window is signal 
or noise only for "non-noisy" channels (see above). This is done by computing the SNR for each receiver within the analysis window. Basically, this SNR is nothing more than the ratio of the short-time average (STA) to long-time average over the particular recorded channel of the data. For example, for the $x$-component of $i$-th geophone, this ratio will be $S_{x i} / L_{x i}$, where $S_{x i}$ is STA over window $W$ for $x$-component of $i$-th geophone, and $L_{x i}$ is LTA over block $B$ for $x$-component of $i$-th geophone. Only if such defined SNR is greater than a preset threshold, the channel registers that the signal is present in the current time window.

A variation on this technique uses as the noise level the STA over the window with minimal energy. Such a window is found by scanning the whole block for the "quietest" window $W_{\min }$ without regard to the particular velocity component (that means one window for all $3 N$ channels). In that case, the SNR in window $W$ for the above example defined as $S_{x i} / S_{\min }$, where $S_{\min }$ is the STA over the window with minimal energy $W_{\min }$.

A detection is declared when at least half of the geophones register a signal in the window $W$ for at least one of the components of the velocity $\left(X_{-}, Y_{-}\right.$or $Z_{-}$ component). If that is not the case, the recorded time series is considered to be noise, and therefore we move to the next analysis window.

\subsubsection{Event localization scheme}

\section{Time delay estimation}

For each event, time delays of the event arrival at different geophones are calculated using a standard cross-correlation procedure. A reference channel is first selected, usually the one where the event arrives first. The signal in that channel is then crosscorrelated with the corresponding signals on all the other geophones on which the event has been detected. The time delay is the time at which the cross-correlation coefficient attains an absolute maximum. 


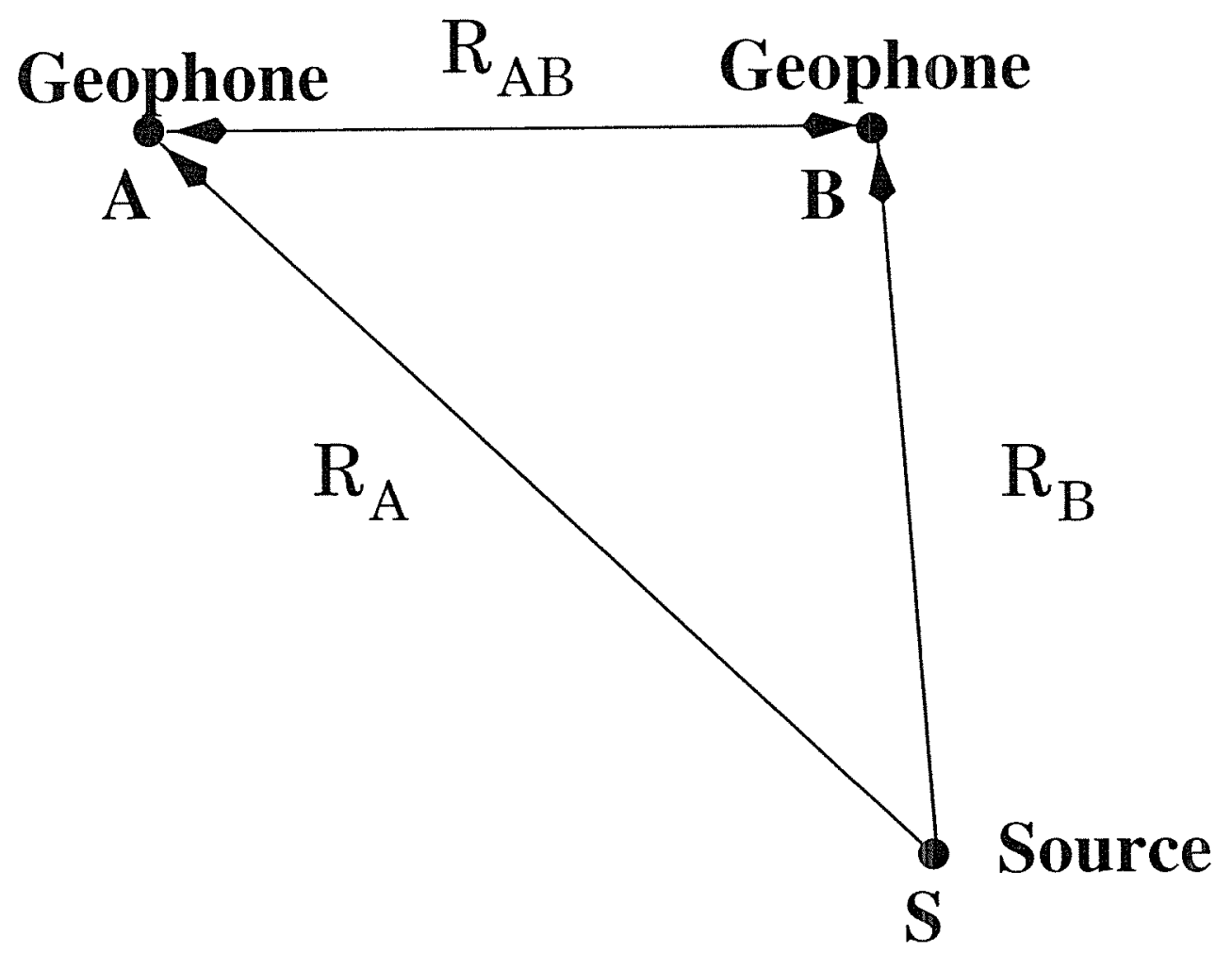

Figure 2-6: Simple receiver configuration for establishing the relations between arrival times. Geophones are located at the points $\mathbf{A}$ and $\mathbf{B}$ and the source at point $\mathrm{S}$. The distance between geophones is $R_{\mathrm{AB}}$. The distance from geophone $\mathrm{A}$ to the source is $R_{A}$, while the distance from geophone $\mathrm{B}$ to the source is $R_{B}$.

The cross-correlation function is defined as

$$
\mathbb{R}_{x_{i}, x_{j}}(\tau)=\mathbb{E}\left[x_{i}(t) x_{j}(t-\tau)\right]
$$

where $\mathbb{E}$ is an expectation operator. Due to finite duration of the time series, only an estimate of $\mathbb{R}$ can be obtained. The value of $\tau$ that maximizes the expression in Equation (2.3) is the time-delay (or arrival time) estimate.

There are some intrinsic checks on the value of delays between the different sensors. Let us consider two sensors (geophones) located at points $\mathbf{A}$ and $\mathbf{B}$ and the source at point $\mathbf{S}$ (see Fig. 2-6). The propagation speed is assumed to be $c_{0}$. Then, according to well-known geometrical inequalities, the distance between the geophones $R_{\mathrm{AB}}$ is not greater than the sum of distances from each geophone to the source (further 

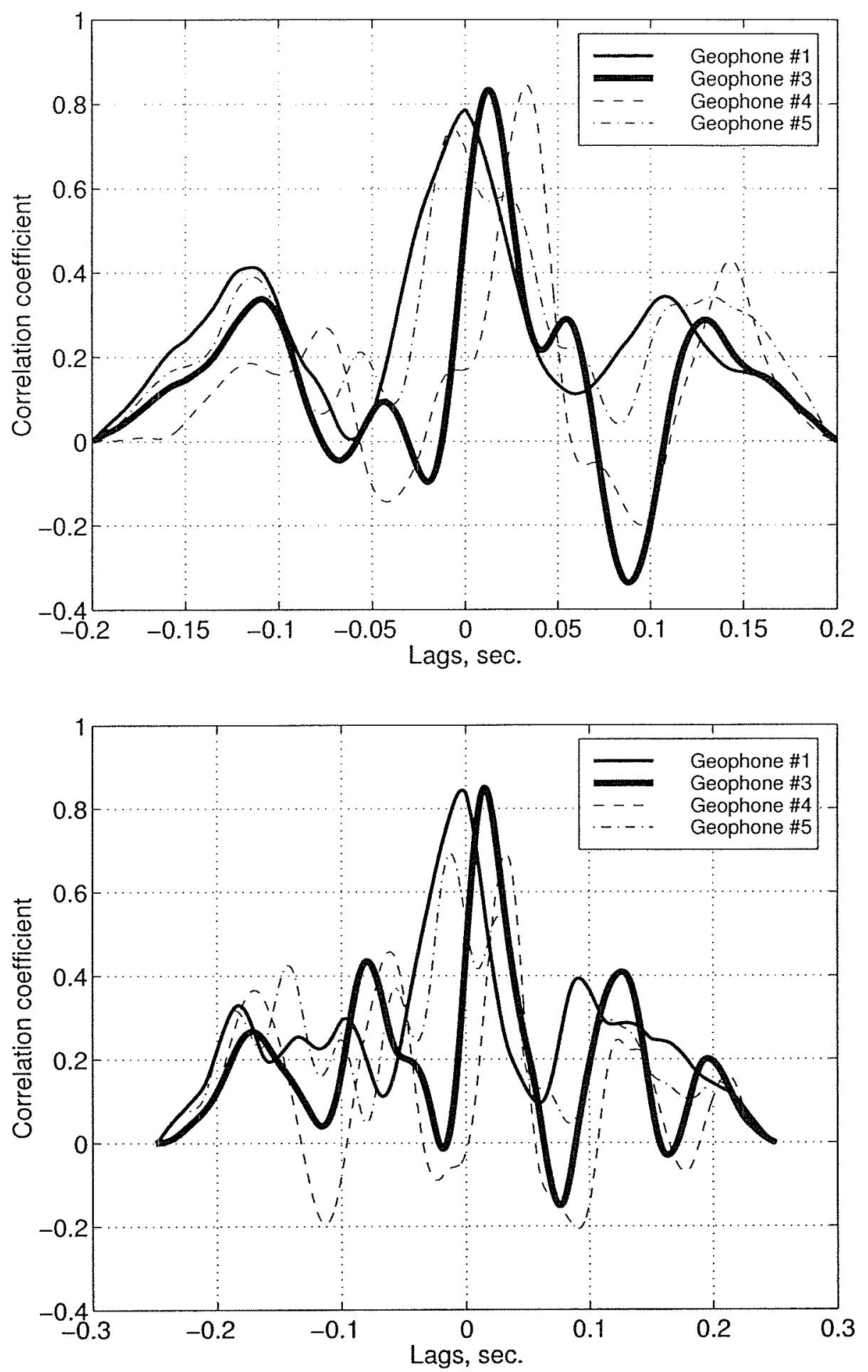

Figure 2-7: Two examples of the cross-correlation between X-components of the geophones. At the top plot the original time series start at $37.5 \mathrm{sec}$. from the beginning of the tape RLAM-26, on the bottom one - at $48 \mathrm{sec}$. from the beginning of the tape RLAM-26. In both cases the cross-correlations were carried out with geophone $\# 2$ being a reference channel. 
referred to as $R_{A}$ and $R_{B}$ ):

$$
R_{\mathrm{AB}} \leq R_{A}+R_{B} .
$$

On the other hand, the absolute value of the difference between the last two ranges is not greater than $R_{\mathrm{AB}}$ :

$$
\left|R_{A}-R_{B}\right| \leq R_{\mathrm{AB}}
$$

Dividing both sides of the last inequality by $c_{0}$, we get:

$$
\left|t_{A}-t_{B}\right| \leq \frac{R_{\mathrm{AB}}}{c_{0}},
$$

where $t_{A}$ and $t_{B}$ are propagation times from the source to the corresponding receiver. The distances between the geophones are on the order of $50 \mathrm{~m}$. For the typical values of compressional and shear wave propagation speeds in ice $(3500 \mathrm{~m} / \mathrm{s}$ and $1800 \mathrm{~m} / \mathrm{s})$, we obtain the following maximum allowed delays between geophones:

$$
\begin{aligned}
& \Delta t_{c} \approx 14 \mathrm{msec} . \\
& \Delta t_{s} \approx 28 \mathrm{msec} .
\end{aligned}
$$

where $\Delta t_{c}$ is the maximum allowed delay between geophones for the compressional waves, and $\Delta t_{s}$ is the maximum allowed delay between geophones for the shear waves.

Two examples of the cross-correlation are shown on Fig. 2-7. In both examples the outputs of $\mathrm{X}$-axes of the geophones were used and geophone \#2 was chosen as a reference channel.

\section{Least-square fit for interarrival times: Search in Cartesian coordinate space}

In this method a rectangular grid is constructed, with cell size $10 \mathrm{~m} \times 10 \mathrm{~m}$. The source is placed at each grid point. The distance from the source location to each 


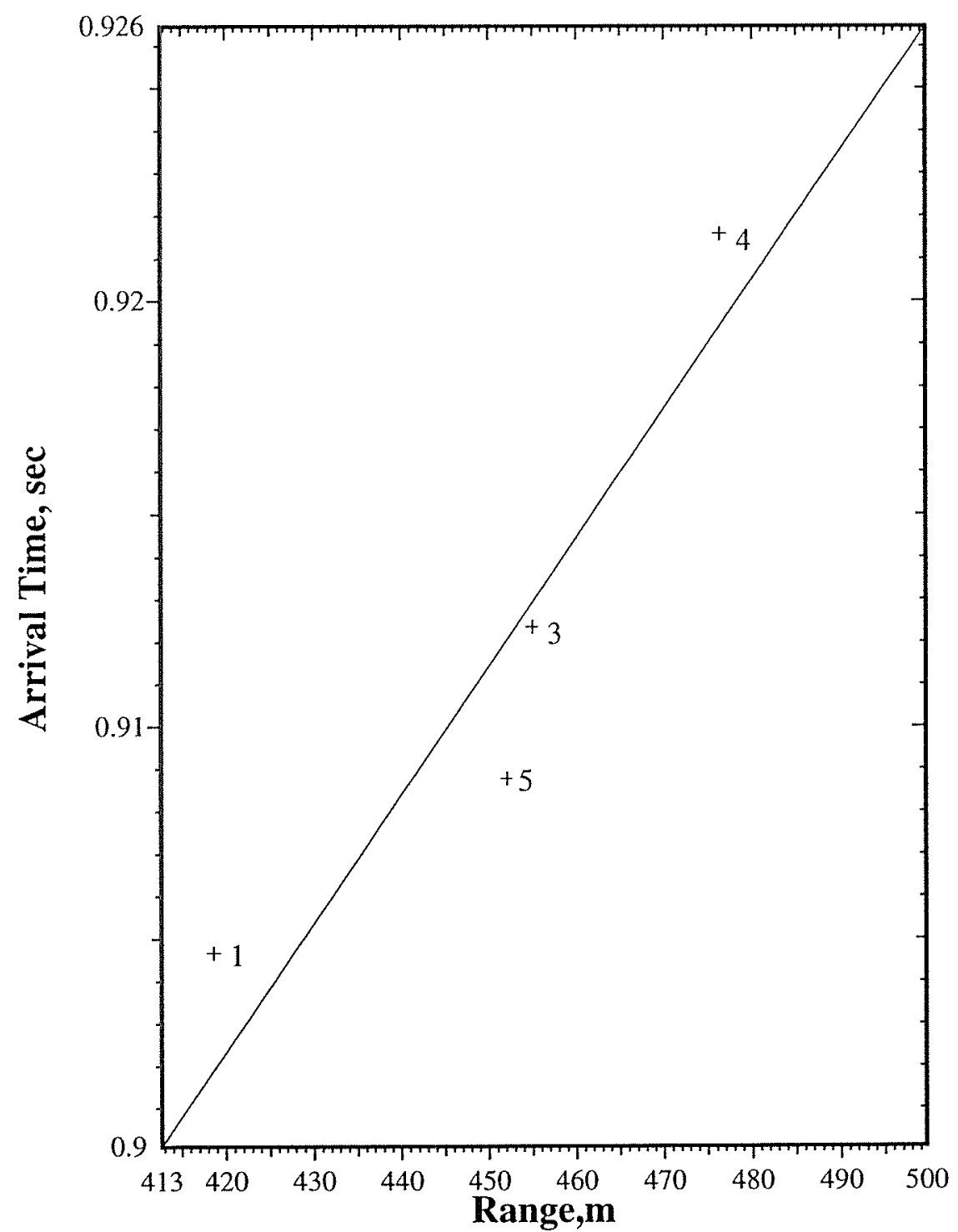

Figure 2-8: Example of the least-square fit to arrival times on 4 geophones. The measured arrival times are plotted versus ranges to the best-fit source location. The location of event was estimated to be $110 \mathrm{~m}$ to the east and $430 \mathrm{~m}$ to the north from the array apex. Standard deviation -0.0022 . Estimated wave speed $-3300 \mathrm{~m} / \mathrm{s}$. 


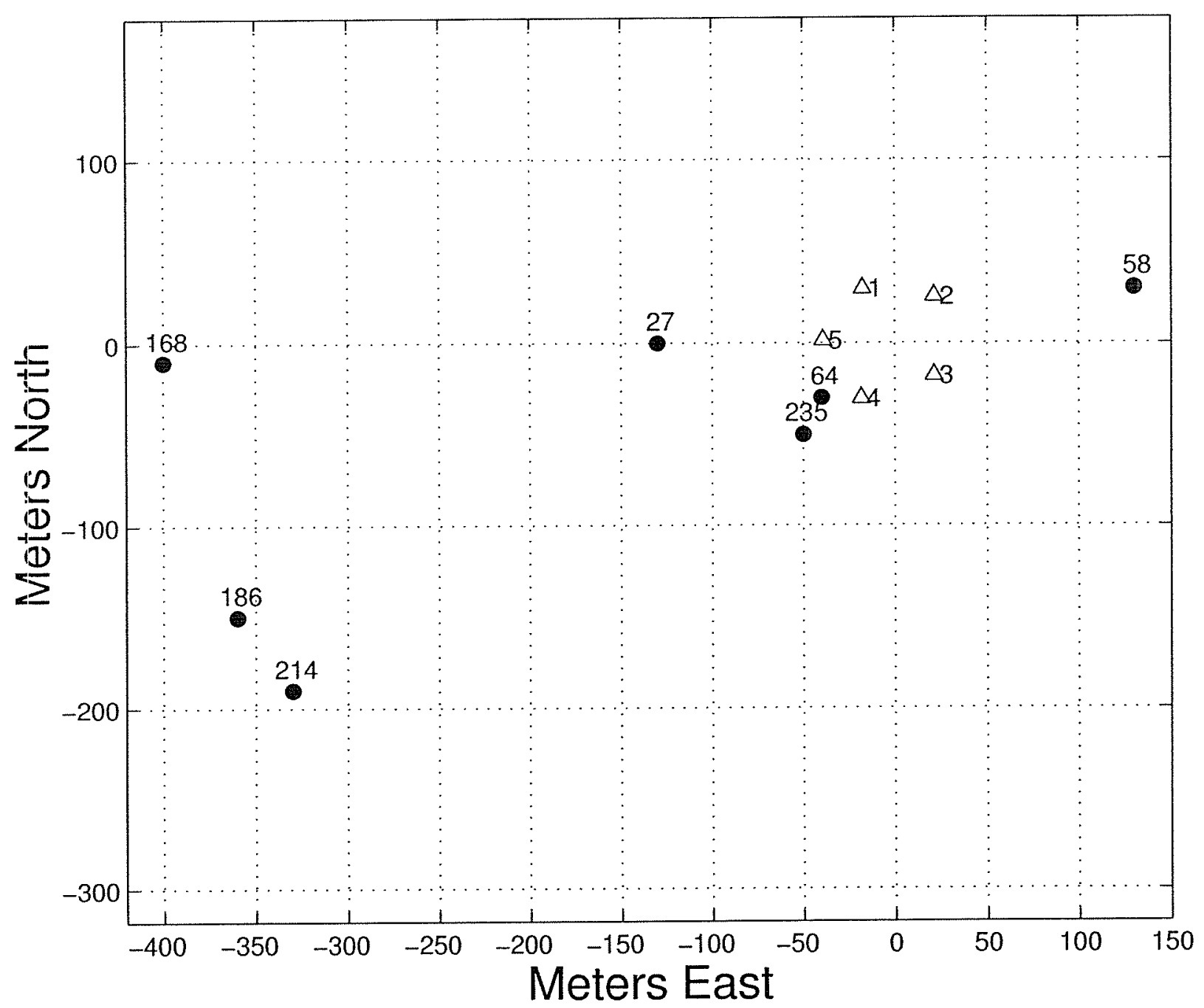

Figure 2-9: Locations of events determined by standard least-square fit. Triangles mark geophone locations, filled circles - event locations. The numbers nearby markers show the moment of time (from the beginning of the RLAM tape 26) at which event occured (rounded to seconds). 
geophone is computed, as well as corresponding time delays. The standard deviation from the measured delay values is then calculated as

$$
\sigma_{\tau}=\sqrt{\frac{\sum_{i=1}^{N}\left(\tau_{i}-\tau_{i}^{\text {(est) }}\right)^{2}}{N}},
$$

where $N$ is the number of the geophones and $\tau_{i}$ and $\tau_{i}^{\text {(est) }}$ are the measured and estimated time delays, respectively. The propagation speed is also estimated as the slope of the best-fit regression line through the points $\left(\tau_{i}^{\text {(est) }}, R_{i}\right)$ with $R_{i}$ being the range for each geophone from the source location. The parameters $A$ and $B$ of the least-square fit $\tau_{i}=A R_{i}+B$, can be determined as

$$
\begin{aligned}
A= & \frac{\sum_{i=1}^{N} \tau_{i} R_{i}-\frac{1}{N} \sum_{i=1}^{N} R_{i} \sum_{i=1}^{N} \tau_{i}}{\sum_{i=1}^{N} R_{i}^{2}-\frac{1}{N}\left(\sum_{i=1}^{N} R_{i}\right)^{2}} \\
B= & \frac{\sum_{i=1}^{N} \tau_{i}-A \sum_{i=1}^{N} R_{i}}{N} .
\end{aligned}
$$

Then for the propagation speed $c_{0}$, we have $c_{0}=1 / A$, and the Equation (2.6) can be rewritten as

$$
\sigma_{\tau}=\sqrt{\frac{\sum_{i=1}^{N}\left(\tau_{i}-A R_{i}-B\right)^{2}}{N}} .
$$

For the example of least-square fit shown on Fig. 2-8, the value for the wave propagation speed was estimated to be $3300 \mathrm{~m} / \mathrm{s}$, which is realistic for typical Arctic ice conditions.

Several events located by this method at the beginning of the RLAM tape 26 are shown on the Fig. 2-9. We see from the figure that events occurring at the moments of time $168 \mathrm{sec}, 186 \mathrm{sec}$ and $214 \mathrm{sec}$ after the beginning of the tape appear to lie on the same arc, almost at the limit of the region of reliable localization by the geophone array. 
Other events at the beginning of the same tape I grouped separately because their waveforms had similar features which repeated from one event to another (see Fig. 2-10). Compare, for example the degradation of the shape of the arrival on the first geophone as compared to other geophones for all shown events. The locations of events corresponding to these time series (including one not shown on Fig. 2-10) are shown on Fig. 2-11. I call this group of 5 events Cluster \#1. In this group all events are at approximately the same azimuth and their locations are quite close to each other (except the last one).

\section{Least-square fit for interarrival times: Search in propagation speed space}

The main difference from the previous method is that the search is carried out in propagation speed space as opposed to the search in Cartesian coordinate space for trial source locations. In this method we try different values of propagation speed and again minimize the standard deviation over the search space. For each trial value of propagation speed $c_{0}$ in the ice plate, the arrival time $t_{i}$ at the $i$-th geophone can be expressed as [20]

$$
t_{i}=t_{s}+\frac{1}{c_{0}} \sqrt{\left(x_{s}-x_{i}\right)^{2}+\left(y_{s}-y_{i}\right)^{2}+\left(z_{s}-z_{i}\right)^{2}}
$$

where $t_{s}$ is the actual moment of time when the ice event located at $\left(x_{s}, y_{s}, z_{s}\right)$ happened. The $N$ geophones are assumed to be positioned at $\left(x_{i}, y_{i}, z_{i}\right)$ with $(i=$ $1, \ldots, N)$. Usually, the geophones are deployed approximately at the same depth (further referred to as $z_{0}$ ).

Multiplying both sides of Equation (2.9) by $c_{0}$, squaring them, and then subtracting from the equations for $(i=2, \ldots, N)$ the equation for first geophone $(i=1)$, 

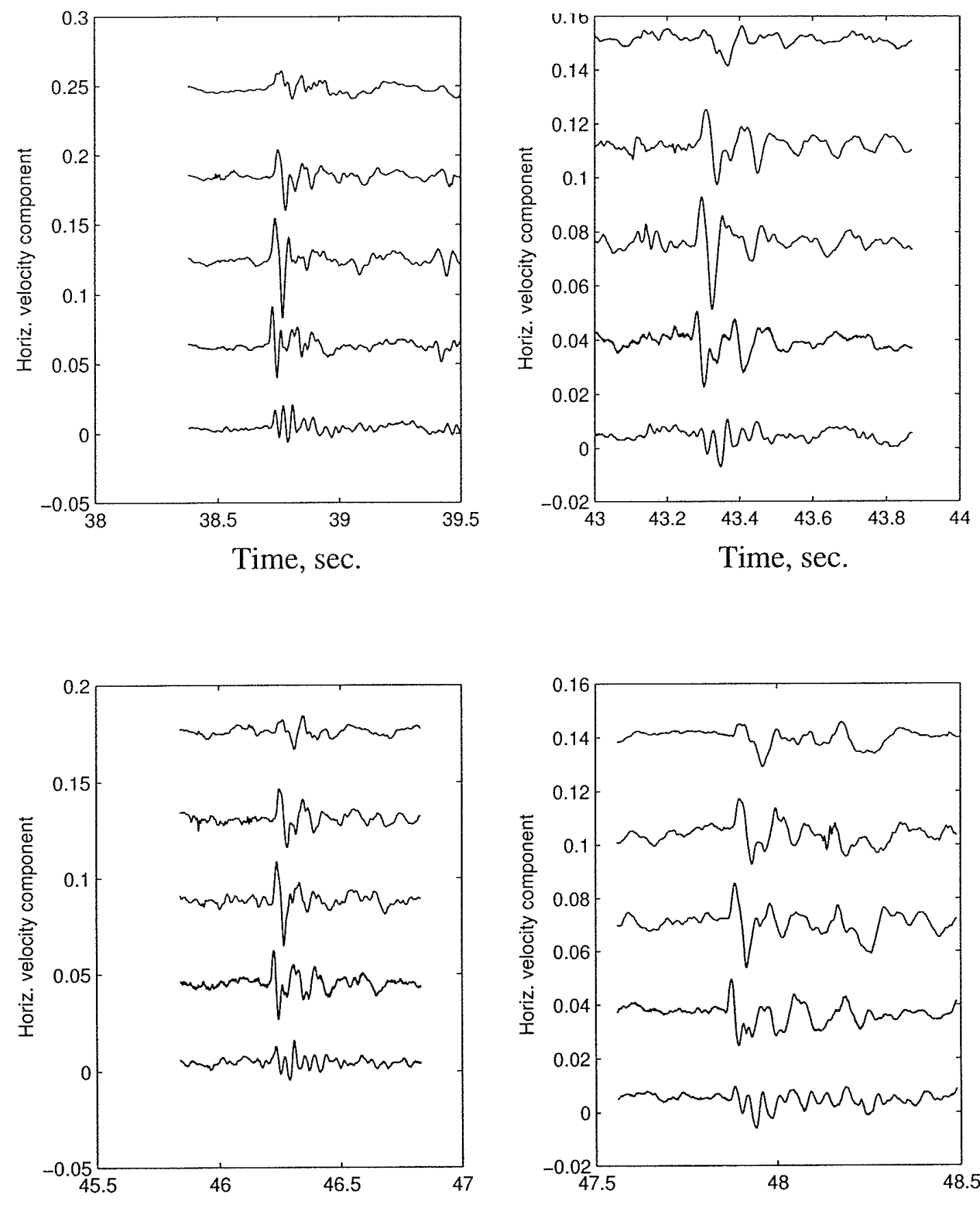

Time, sec.

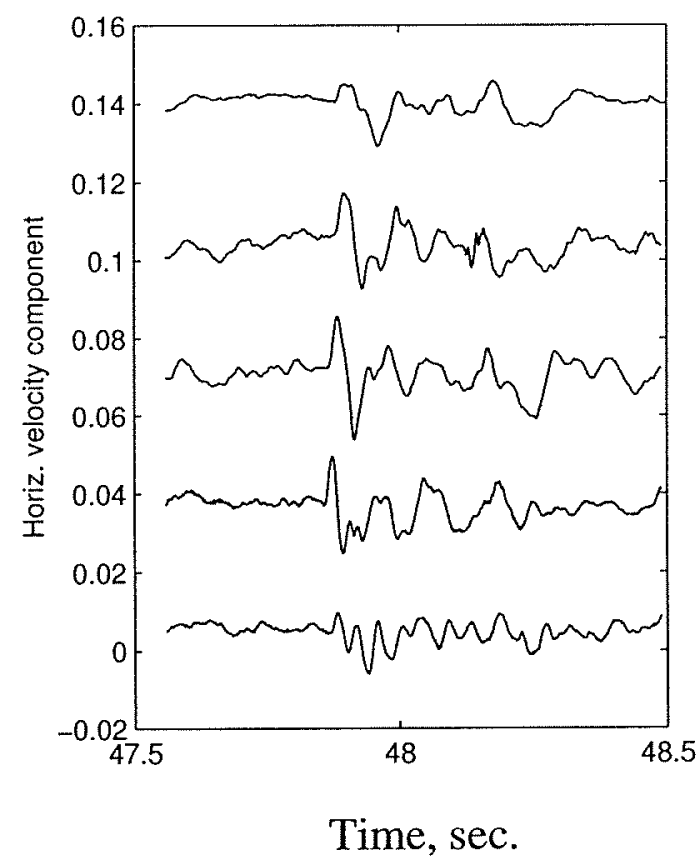

Figure 2-10: Stacked time series of the X-component (North) of the velocity for four events at the beginning of the RLAM tape 26. The time series corresponding to the geophone $\# 1$ are at the top. All events have strikingly similar features in their waveforms. Compare the degradation of the shape of the arrival on the first geophone as compared to others for all shown events. 


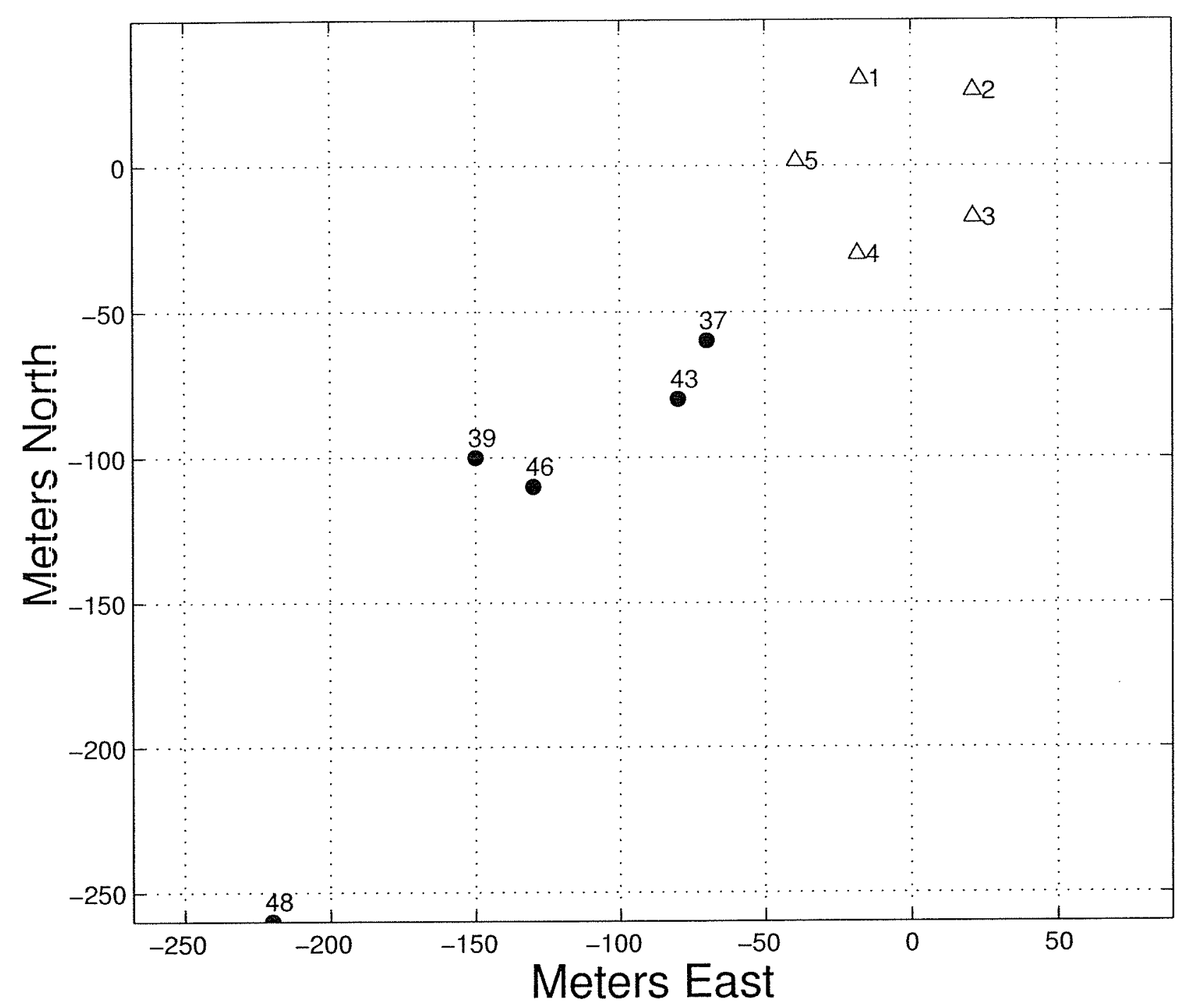

Figure 2-11: Locations of events time series for which have striking similarities (compare Fig. 2-10). Triangles mark geophone locations, filled circles - event locations. The numbers nearby markers show the moment of time (from the beginning of the RLAM tape 26) at which event occured (rounded to seconds). In this group all events are at approximately the same azimuth and their locations are quite close to each other (except the last one). 
we obtain the following system of $(N-1)$ equations:

$$
\left[\begin{array}{ccc}
2\left(x_{2}-x_{1}\right) & 2\left(y_{2}-y_{1}\right) & -2 c_{0}^{2}\left(t_{2}-t_{1}\right) \\
\vdots & \vdots & \vdots \\
2\left(x_{N}-x_{1}\right) & 2\left(y_{N}-y_{1}\right) & -2 c_{0}^{2}\left(t_{N}-t_{1}\right)
\end{array}\right]\left\{\begin{array}{c}
x_{s} \\
y_{s} \\
t_{s}
\end{array}\right\}=\left\{\begin{array}{c}
\left(x_{2}^{2}+y_{2}^{2}-c_{0}^{2} t_{2}^{2}\right)-s_{1}^{2} \\
\vdots \\
\left(x_{N}^{2}+y_{N}^{2}-c_{0}^{2} t_{N}^{2}\right)-s_{1}^{2}
\end{array}\right\}
$$

where

$$
s_{1}=\sqrt{x_{1}^{2}+y_{1}^{2}-c_{0}^{2} t_{1}^{2}}
$$

So we need to solve this system of $(N-1)$ equations for three unknowns $x_{s}, y_{s}$ and $t_{s}$. In matrix notation the above system takes the form of

$$
\mathrm{A} \vec{X}=\vec{B}
$$

where matrix A contains all coefficients from the lefthand side of the system of equations, the vector $\vec{X}$ contains all unknowns and the vector $\vec{B}$ contains all coefficients from the righthand side of the original system above.

The solution of Equation (2.10) can be obtained using the least-square solution described in Appendix A:

$$
\vec{X}=\left(\mathbf{A}^{T} \mathbf{A}\right)^{-1}\left(\mathbf{A}^{T} \vec{B}\right)
$$

Note that this type of solution works only when the matrix $\left(\mathbf{A}^{T} \mathbf{A}\right)$ is non-singular (i.e. it has an inverse). Under that condition, we can determine the corresponding source location $\left(x_{s}, y_{s}\right)$ and the standard deviation between calculated and exper:mental interarrival times (defined similarly to Equation (2.6)) for each value of trial propagation speed $c_{0}$. The location of the source at the output of this method will be the location corresponding to the value of the trial propagation speed $c_{0}$ which minimizes the standard deviation between calculated and experimental interarrival times. 


\subsection{Flexural wave arrival}

In this section some results for the flexural wave arrival will be discussed. First, the method for determining dispersion characteristics of the arrival, the multiple filter technique (further referred to as MFIt), will be described. After that, the dispersion pattern of the flexural wave arrival determined by MFlt method will be discussed. Finally, the sense of motion in flexural waves will be presented based on velocity/displacement hodographs of motion in the flexural wave arrival.

\subsubsection{Multiple filter technique}

The first step in the multiple filter technique by Dziewonski et al. [9] is to pass the data through a bank of Gaussian narrowband filters covering the frequency range of interest. The authors use Gaussian filters in order to obtain an optimal balance between frequency and time resolution. The general expression for one of such filters in the frequency domain is

$$
H_{n}(\omega)=e^{-\alpha\left(\frac{\omega-\omega_{n}}{\omega_{n}}\right)^{2}}
$$

where $\omega_{n}$ is the center frequency of the $n$-th filter and $\alpha$ is the parameter that controls the resolution of the filter. In original paper, a filter bank with constant relative bandwidth was used, but here it was more convenient to use filters with constant bandwidth.

In the next stage of this method, the analytic signal for each frequency band used is calculated. The analytic signal is defined as:

$$
\begin{aligned}
& \hat{S}\left(t ; \omega_{c}\right)=s\left(t ; \omega_{c}\right)+j \mathbf{H}\left\{s\left(t ; \omega_{c}\right)\right\} \\
& \hat{S}\left(t ; \omega_{c}\right)=A\left(t ; \omega_{c}\right) e^{j \phi\left(t ; \omega_{c}\right)}
\end{aligned}
$$

where:

$\omega_{c}$ - center frequency of the narrowband filter, 
$s\left(t ; \omega_{c}\right)$ - filtered signal,

H - Hilbert transform,

$\hat{S}\left(t ; \omega_{c}\right)$ - analytic signal,

$A\left(t ; \omega_{c}\right)$ - instantaneous amplitude of the signal,

$\phi\left(t ; \omega_{c}\right)$ - instantaneous phase of the signal.

Then, the group arrival time for the center frequency of each band is estimated

as the time at which the maximum of the complex envelope (or the absolute value of the analytic signal) occurs. The group arrival time can be expressed as

$$
t=\frac{R}{V_{g}}
$$

where:

$R$ - distance from source to receiver,

$V_{g}$ - group velocity.

Using the above expression, we can now estimate the group velocity of the signal.

\subsubsection{Flexural wave dispersion}

Fig. 2-12 shows the location of the event that produced vertical velocities characteristic of a flexural wave dispersion pattern (first arrive higher frequencies, later lower frequencies). The time series of vertical velocities for this event are shown on Fig. 2-13. These times series were processed using the MFIt technique. The result of this processing for the geophone \#5 is shown on Fig. 2-14. On this plot the arrival times were converted to group velocities using the distance from the geophone to the event location and the time the event occured, obtained previously.

The general trend can be seen even on this individual plot, but quantitative results are better obtained from averaging across geophones. This is done in the following way: the arrival time corresponding to the maximum spectral amplitude at each frequency for each geophone separately is determined, this time is converted to 


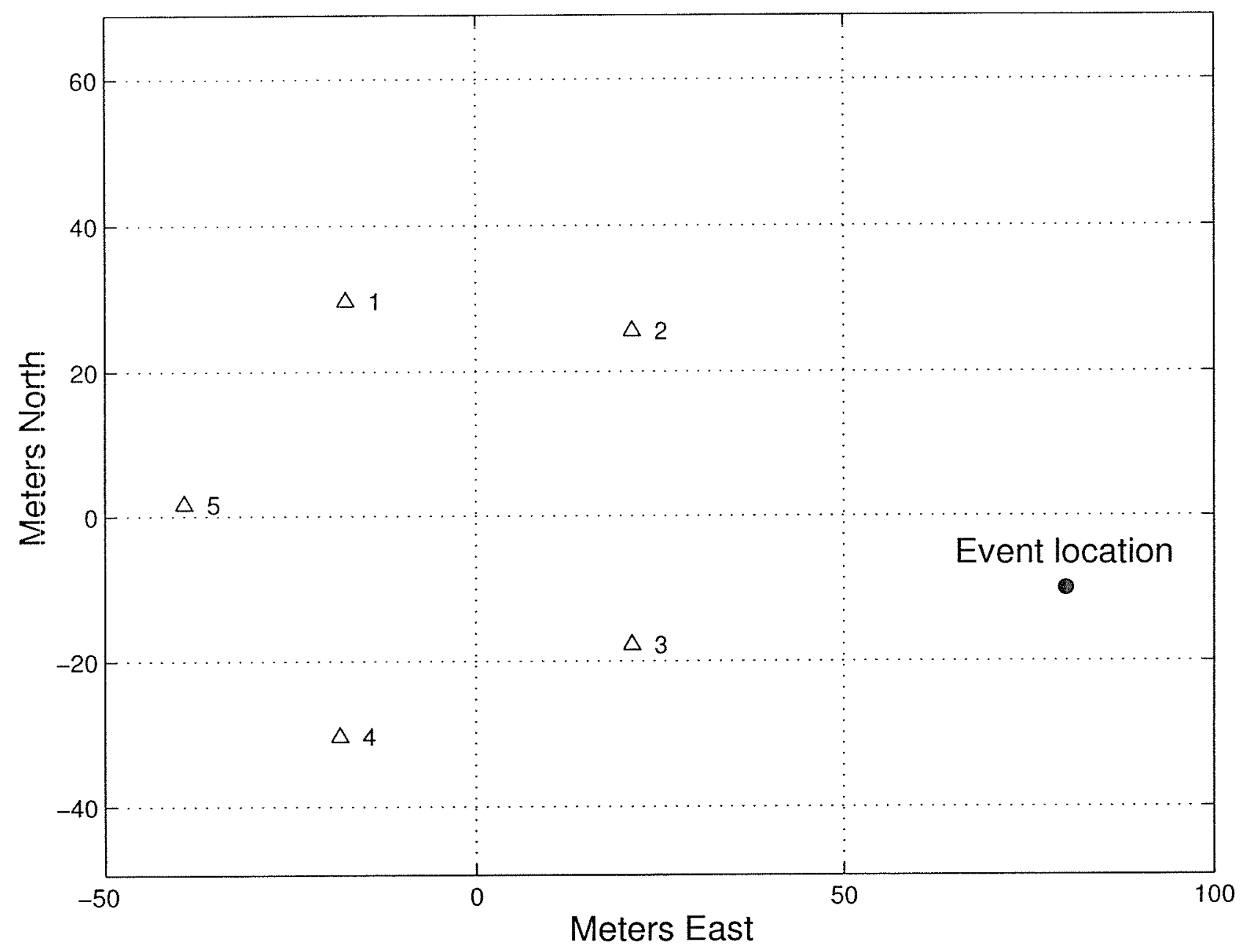

Figure 2-12: Location of the event that produced the vertical velocities characteristic for the flexural wave dispersion pattern (first arrive higher frequencies, later - lower frequencies). Event location is shown by filled circle marker, while geophone locations are shown by unfilled triangles. This event location was determined using the least-square fit for the arrival times determined by cross-correlation of the vertical component of the velocity registered by the geophones. 


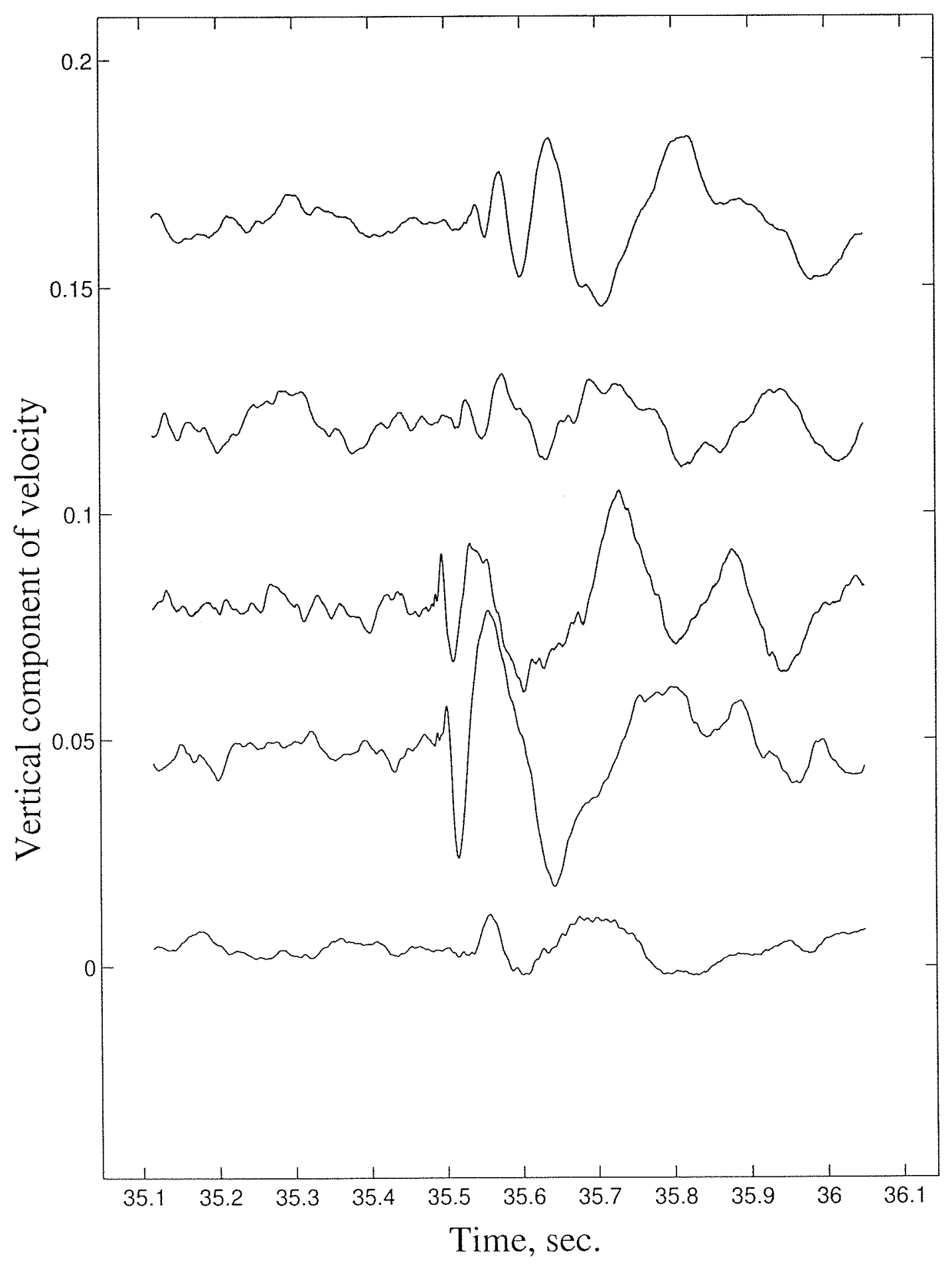

Figure 2-13: Stacked time series of vertical velocities for the event that produced the vertical velocities characteristic for the flexural wave dispersion pattern (first arrive higher frequencies, later - lower frequencies). The time series recorded by the first geophone are at the bottom, while time series corresponding to the last geophoneat the top. 


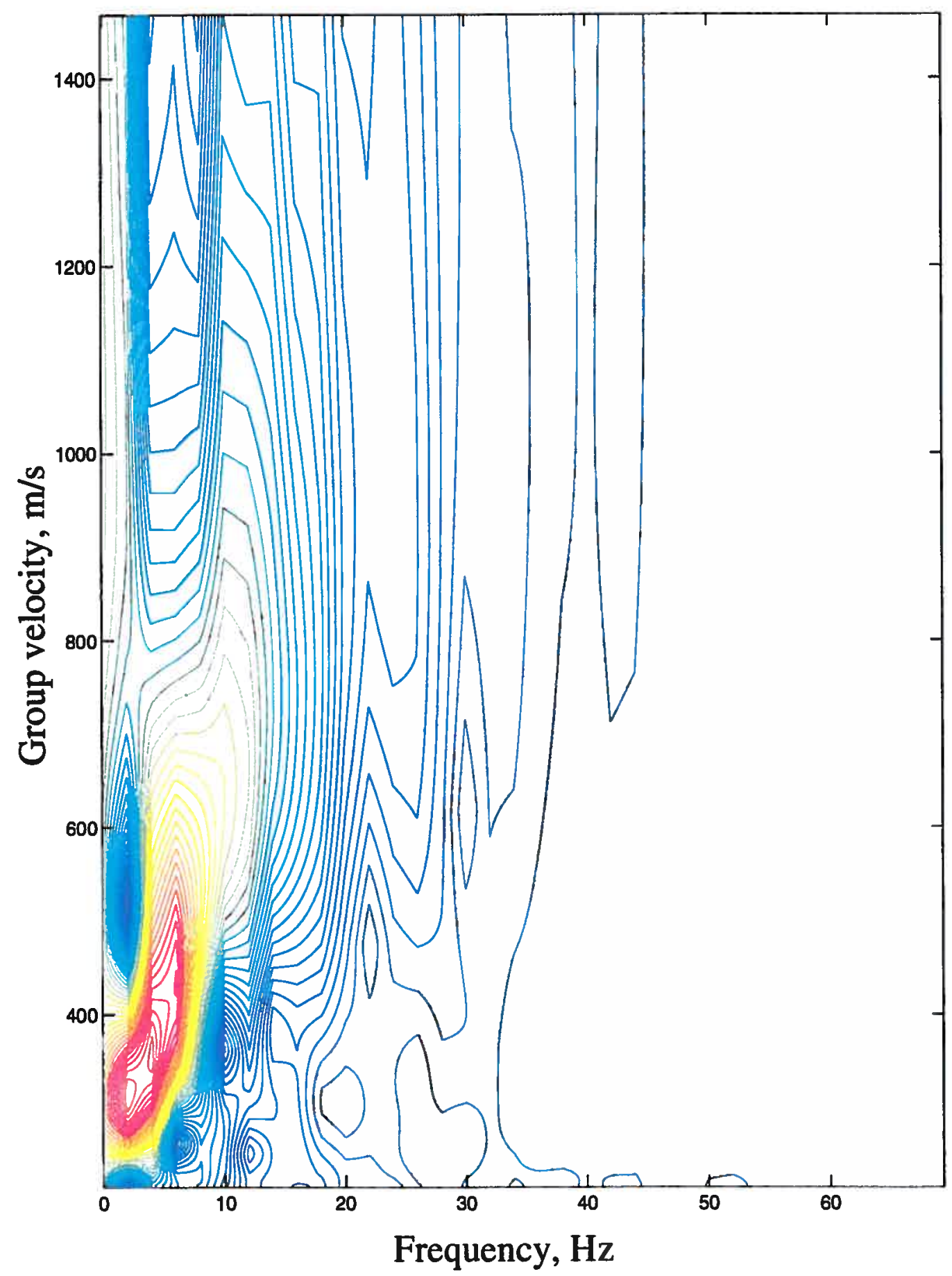

Figure 2-14: Results of the processing of the vertical velocity component recorded by the geophone \#5 using MFIt technique. The group velocities were obtained as a result of arrival times conversion using known range to the source (see Fig. 2-12). 


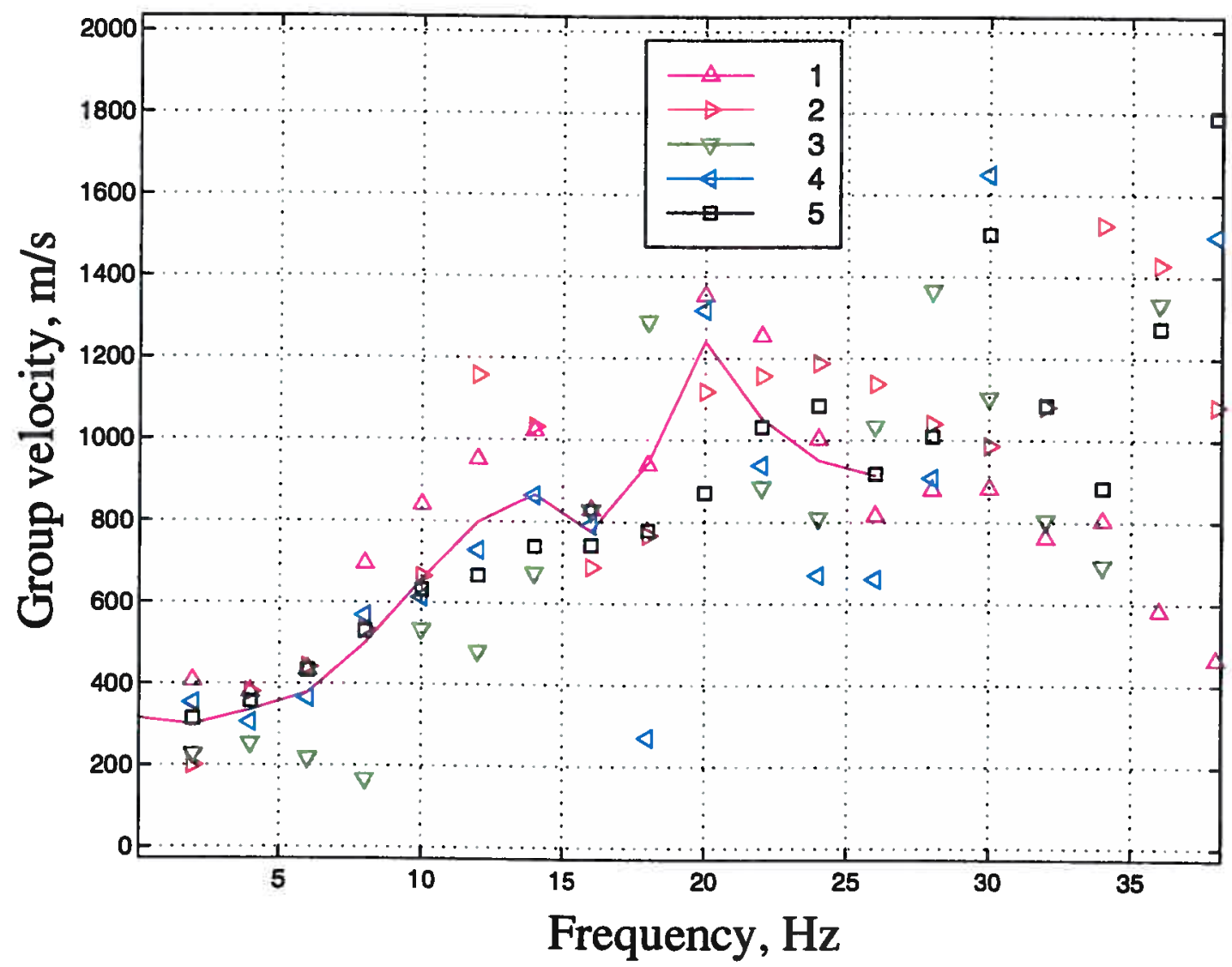

Figure 2-15: Dispersion curves of flexural wave. Five different type of markers correspond to different geophones (see legend). Average across geophones is shown on this plot as a solid line. Note, that these results should be believed only till about $20 \mathrm{~Hz}$, because higher frequencies are absent in time series, as it can be seen from the Fig. 2-13.

the group velocity using the known range to source, then 5 different curves (different markers corresponding to different geophones) are plotted as group velocity versus frequency (see Fig. 2-15). The average across geophones is shown on this plot as a solid line (in one case, at $18 \mathrm{~Hz}$, I removed one outlier). This dispersion curve should be believed only until about $20 \mathrm{~Hz}$, because higher frequencies are absent in time series, as can be seen from the Fig. 2-13. 


\section{Rolling ball}

\section{Direction of propagation}
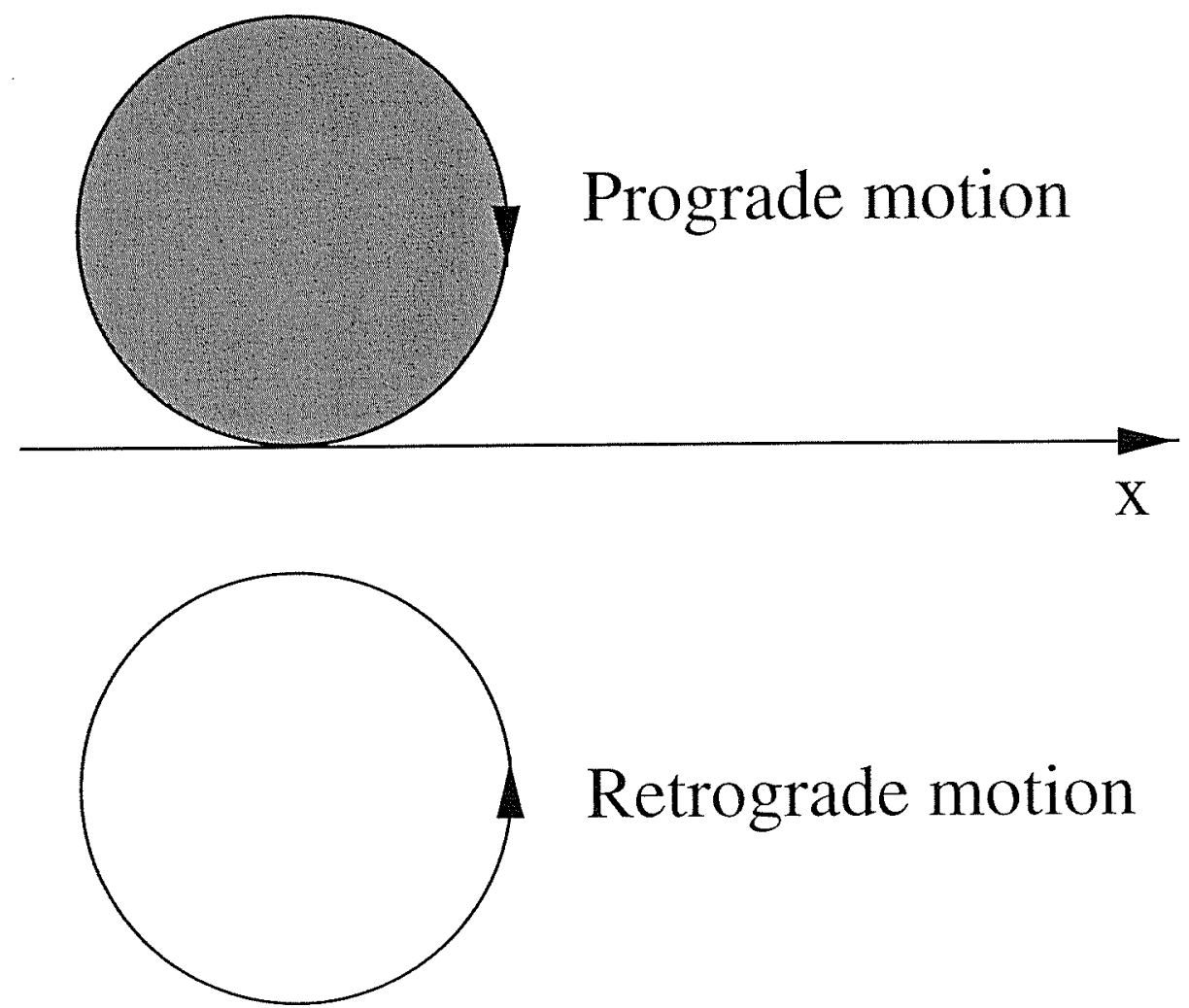

Figure 2-16: Prograde and retrograde senses of the particle motion. According to [1], the convention for distinguishing between prograde and retrograde motion can perhaps best be remembered in terms of a ball rolling along the top of the half-space from source to receiver. The sense of rotation of the ball is prograde, while for the Rayleigh wave at the surface the particle motion is retrograde.

\subsubsection{Sense of motion in flexural wave}

The different modes of particle motion can be distinguished by their so called "sense of particle motion". According to [1], the convention for distinguishing between prograde and retrograde particle motion can perhaps best be remembered in terms of a ball rolling along the top of the half-space from source to receiver (see Fig. 2-16). When the wave is propagating to the right, prograde sense of motion corresponds to clockwise motion, while retrograde sense - counterclockwise. For the fundamental mode of the Rayleigh wave at the surface, the sense of particle motion is retrograde.

Fig. 2-17 shows the hodographs of velocity and displacement on one of the 


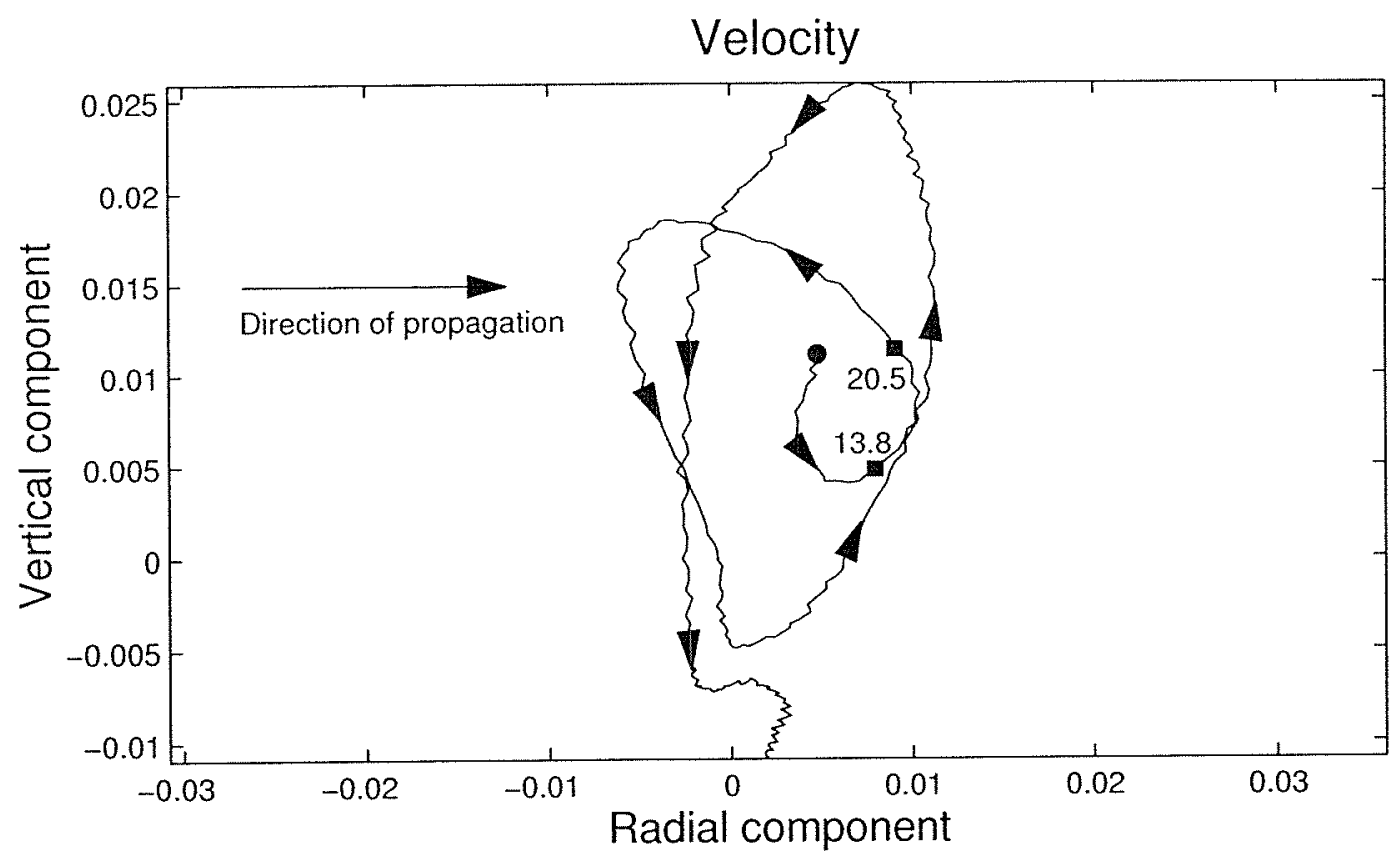

Displacement

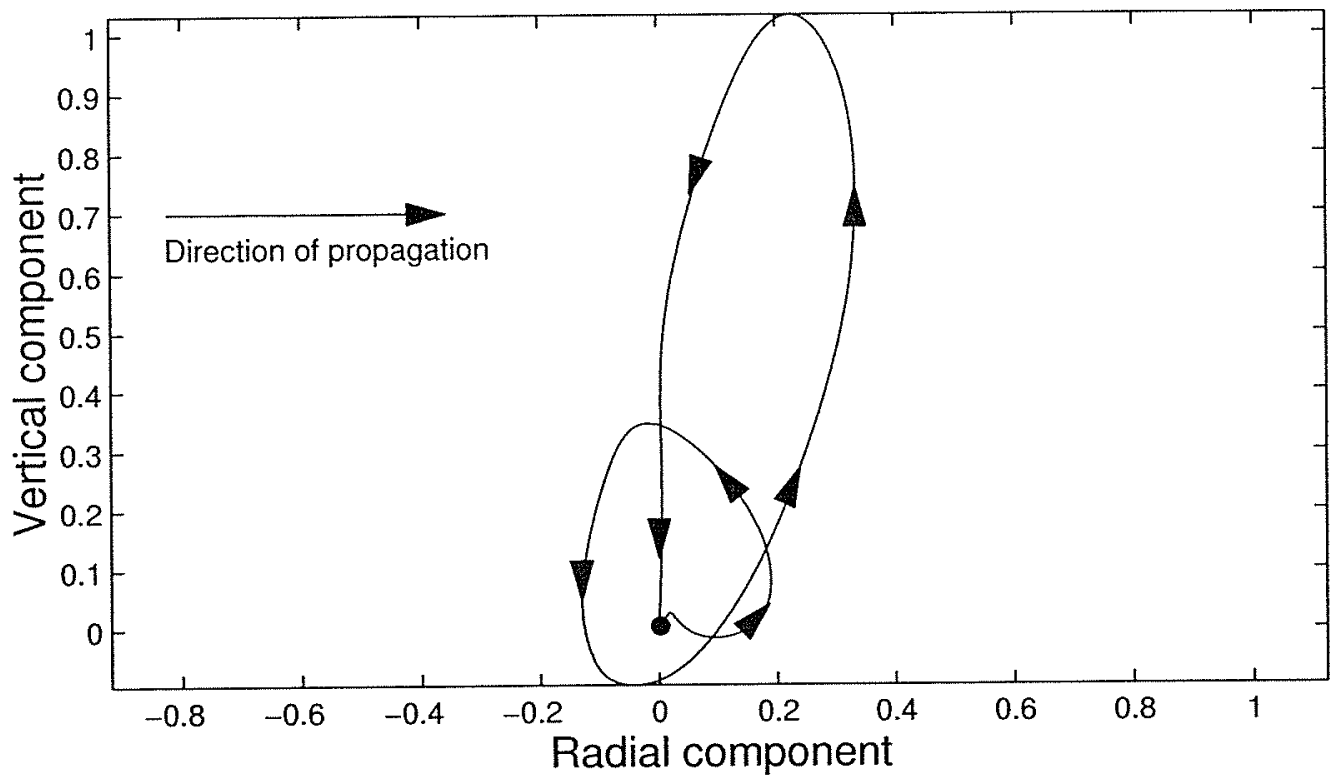

Figure 2-17: Hodographs of velocity and displacement in the flexural wave arrival for geophone \#5. Top plot shows hodograph of velocity, while the bottom plot the hodograph of displacement. Each hodograph shows vertical component of motion (velocity or displacement) versus radial component of motion (in the direction away from the source). The circle markers correspond to the start of the time interval visualized on the hodographs. On the hodograph of velocity (top plot) two additional moments in time are marked on plot by filled squares to resolve ambiguity of hodograph behavior near its first self-intersection. Numbers near markers show approximate times in msec from the start of the plotting interval. 


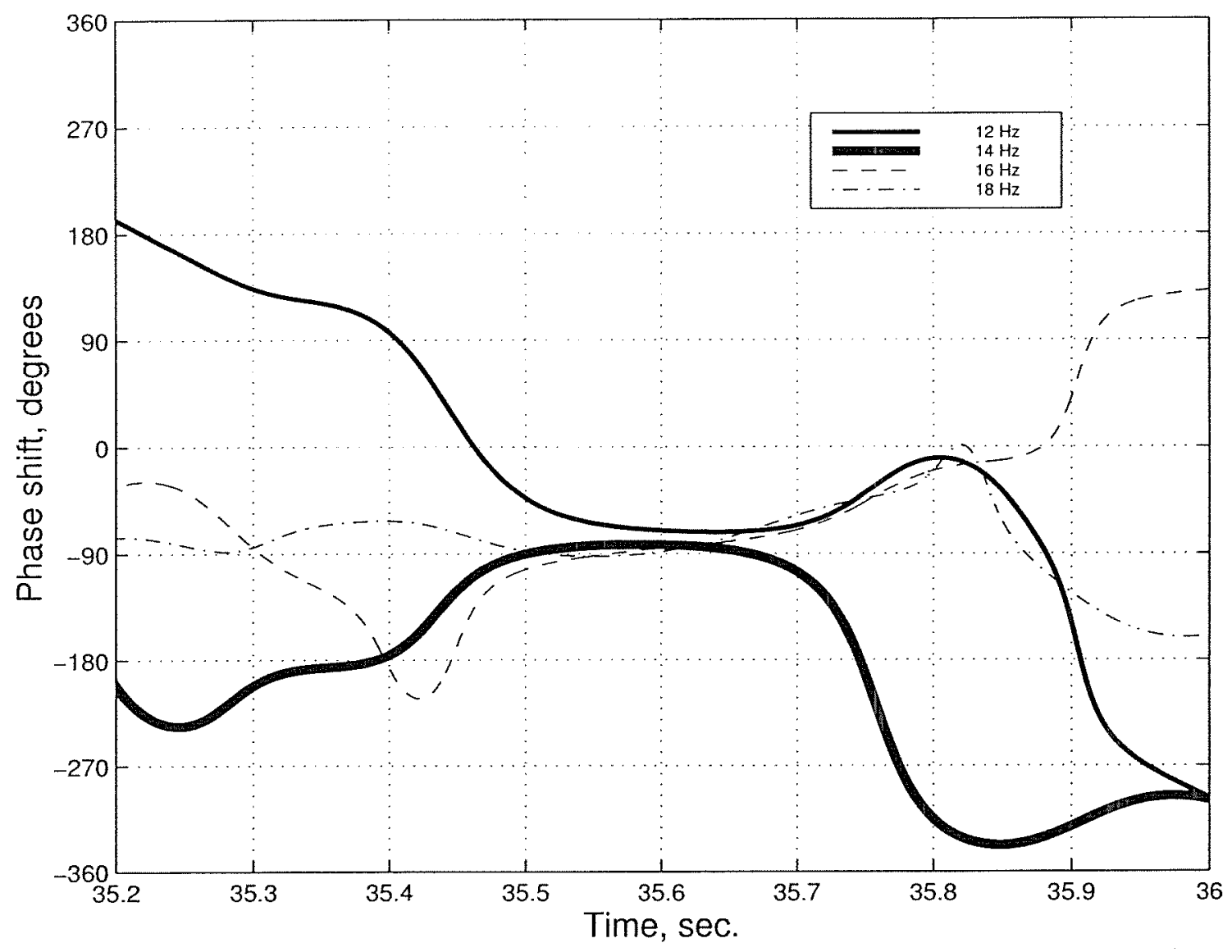

Figure 2-18: Phase shift between vertical and radial components of motion recorded by the geophone \#5. Each curve shows the time dependence of the phase shift for different frequency (from 12 to $18 \mathrm{~Hz}$ ). Note, that all the curves show the phase shift close to $-90^{\circ}$ at time interval between $35.5 \mathrm{sec}$ and $35.7 \mathrm{sec}$. This phase difference corresponds to the retrograde sense of motion, because vertical component is behind of radial component for quarter of period or, in other words, $-90^{\circ}$.

geophones during the flexural wave arrival discussed above. Both hodographs are for motion in vertical radial plane. ${ }^{5}$ The circle markers correspond to the start of the time interval visualized on the hodographs. The direction of the wave propagation is shown on the plot with horizontal arrows pointing to the right. The arrows on the hodographs show the sense of motion. In this case, it appears to be retrograde motion as for the fundamental mode of a flexural wave (the only mode that could possibly exist at such low frequencies).

The phase differences between vertical and radial components of motion on

\footnotetext{
${ }^{5}$ I use this name to denote the vertical plane containing both the receiver and the source.
} 
several frequencies confirm the retrograde sense of motion in this flexural wave arrival (see Fig 2-18). On this figure the different lines correspond to different frequencies (from 12 to $18 \mathrm{~Hz}$, see the legend). All curves on this plot are in the vicinity of $-90^{\circ}$ phase shift at the time interval between $35.5 \mathrm{sec}$ and $35.7 \mathrm{sec}$. This phase difference corresponds to the retrograde sense of motion, because the vertical component lags the radial component by 90 degrees. 


\section{Chapter 3}

\section{Polarization processing}

\subsection{Overview}

Polarization methods are widely used in studies of vector wave fields to exploit the specific relations between characteristics of wave motion in orthogonal directions. In earthquake seismology and geophysical prospecting polarization processing is used to separate physically different wave types in the Earth crust. The same purpose could be achieved for ice events by adapting the methods of polarization processing from earthquake mechanics. The specific method chosen for adaptation, so called "Motion Product Detector" (MPD) method, is described in the first section of this chapter. Two schemes of data processing in this method are designed to isolate waves with predominantly rectilinear and elliptical type of particle motion respectively. The details of the implementation of the MPD method for ice event data processing are discussed in the second section. As one of the byproducts of data processing in this method the bearing of the source is also obtained. Using this information the source location can also be determined through a triangulation procedure. The results of the discrimination between different wave types and event localization are discussed in the next section. The last section is devoted to the summary and conclusions for the polarization processing results. 


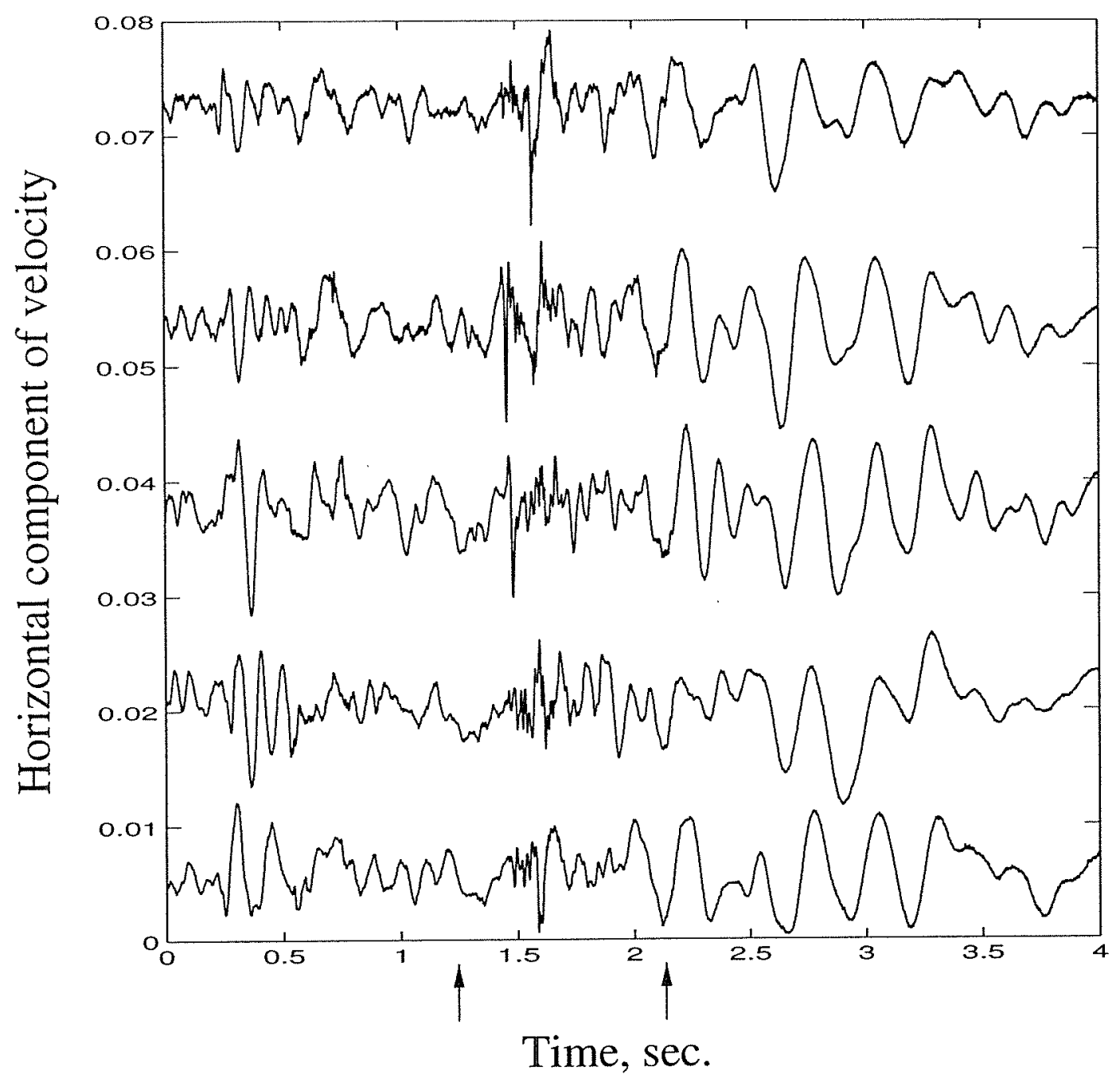

Figure 3-1: Stacked time series of the horizontal (X) component of velocity on 5 geophones which serves as an example of the case when cross-correlation of time series performs poorly.

\subsection{Motion Product Detector (MPD) seismogram method}

As it was already mentioned, one of the first stages in the processing of the recordings of the natural ice events is localization and identification (or discrimination) of the events. The traditional way to do localization by least-square fit to the arrival times on the different geophones occasionally fails, probably, due to overlapping of the different wave types or waves generated by different ice events (see, for example, 


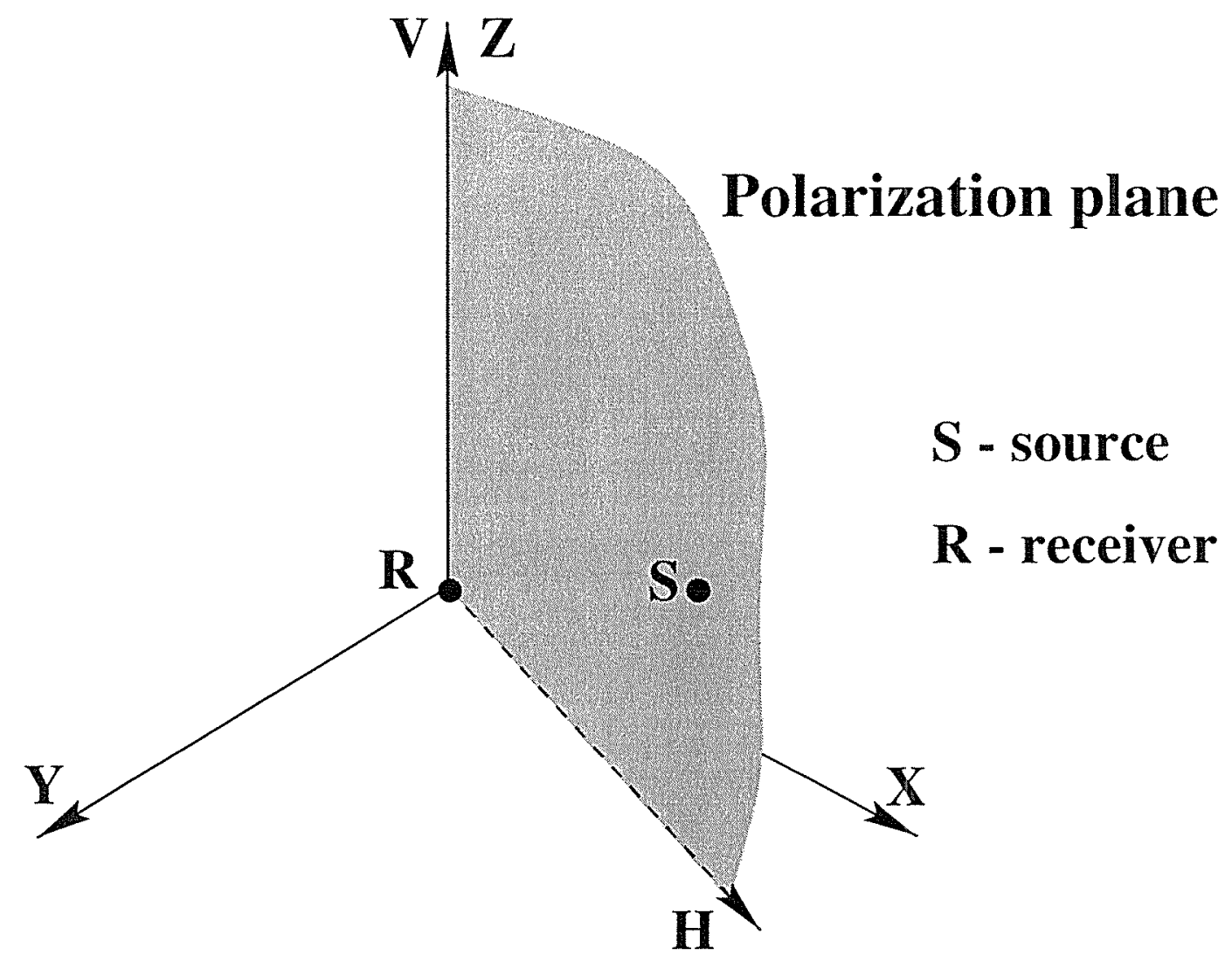

Figure 3-2: Plane of polarization of signals considered in MPD processing. This vertical plane contains both the source and the receiver.

Fig. 3-1). In these cases it is sometimes possible to achieve localization using the Motion Product Detector (MPD) seismogram method inspired by J.E. White's original work [53]. This method allows to isolate vertically polarized waves (for definition of their polarization plane see Fig. 3-2) with two kinds of particle motion trajectories: rectilinear and elliptical (see Fig. 3-3). In the seismology literature the term "polarization" is used to distinguish between these two kinds of particle motion. The difference between rectilinear polarization and elliptic polarization is the phase shift of $\pi / 2$ between the vertical and horizontal components of motion in the latter case, as compared to no phase shift at all for rectilinear polarization. Usually, body waves have rectilinear polarization, while surface waves (e.g. Rayleigh) and flexural waves have elliptic polarization [17], but due to interaction with the free surface of the ice sheet sometimes SV-waves acquire elliptic polarization. So the ability to separate these two polarizations would allow to discriminate between different wave types. 
Rectilinear polarization

(e.g. compressional wave)

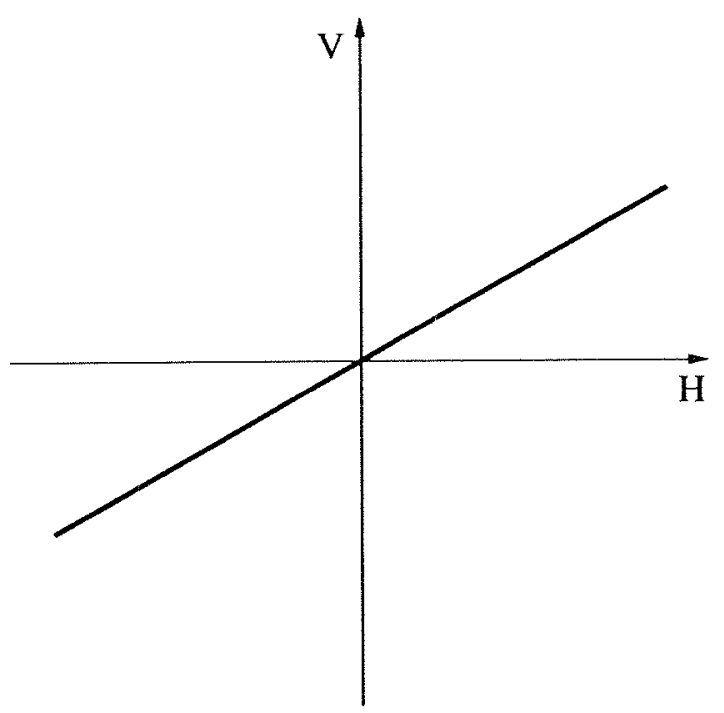

\section{Elliptical polarization}

(e.g. SV wave)

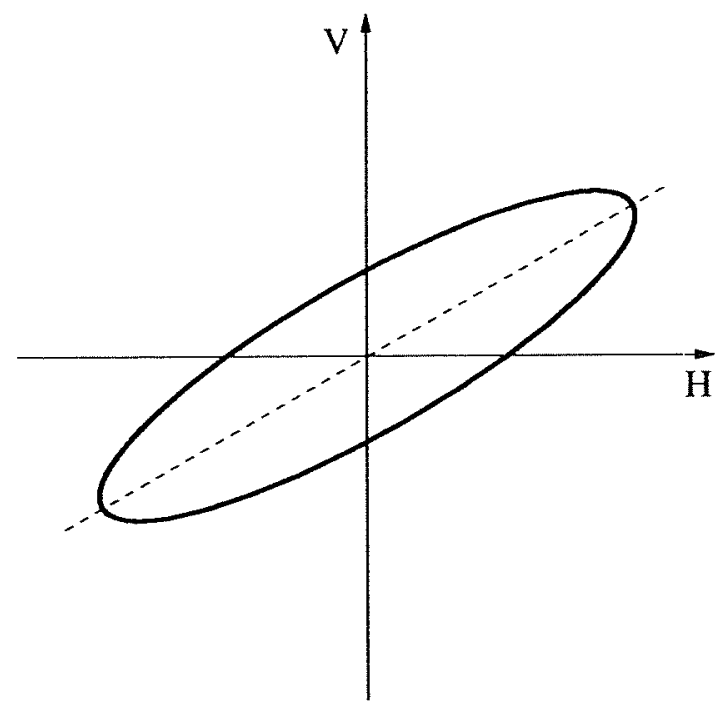

Figure 3-3: Two different kinds of polarization of seismo-acoustic signals in the vertical plane: rectilinear and elliptical motion. The first one is characterized by in-phase horizontal and vertical components of motion, the second by the phase shift of $\pi / 2$ between these components.

\subsubsection{Procedure for isolating rectilinear polarization in data recordings by the MPD method}

To separate the compressional wave each of the horizontal components of ice motion registered by geophone is multiplied by the vertical component and averaged by integration over a certain time window (see Fig. 3-4a). The optimal window length was determined empirically to be $20 \mathrm{msec}$. The result of averaging the product of the horizontal $X$-component with the vertical $Z$-component is called $X_{\mathrm{HV}}$, while the result for the horizontal $Y$-component is called $Y_{\mathrm{HV}}$. To visualize the processing results $Y_{\mathrm{HV}}$ is plotted versus $X_{\mathrm{HV}}$. As a result of the time averaging we obtain an enhanced output from the compressional wave due to the in-phase horizontal and vertical components of motion produced by that wave, while the motion produced by other waves will be substantially attenuated due to the different phase relationship between the vertical and horizontal components in those waves.

So in the case when the compressional wave is present, the output of this HV 


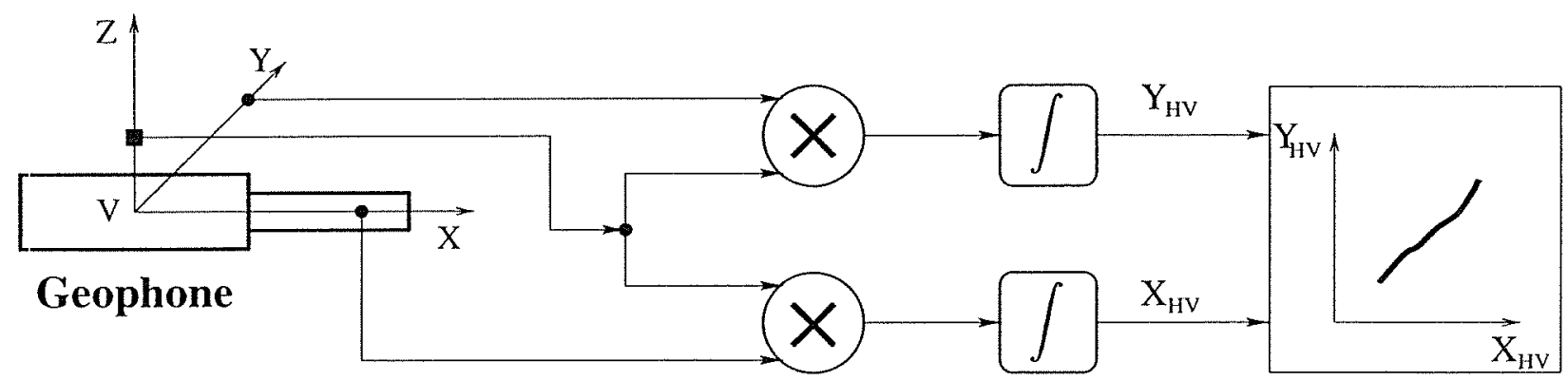

(a)

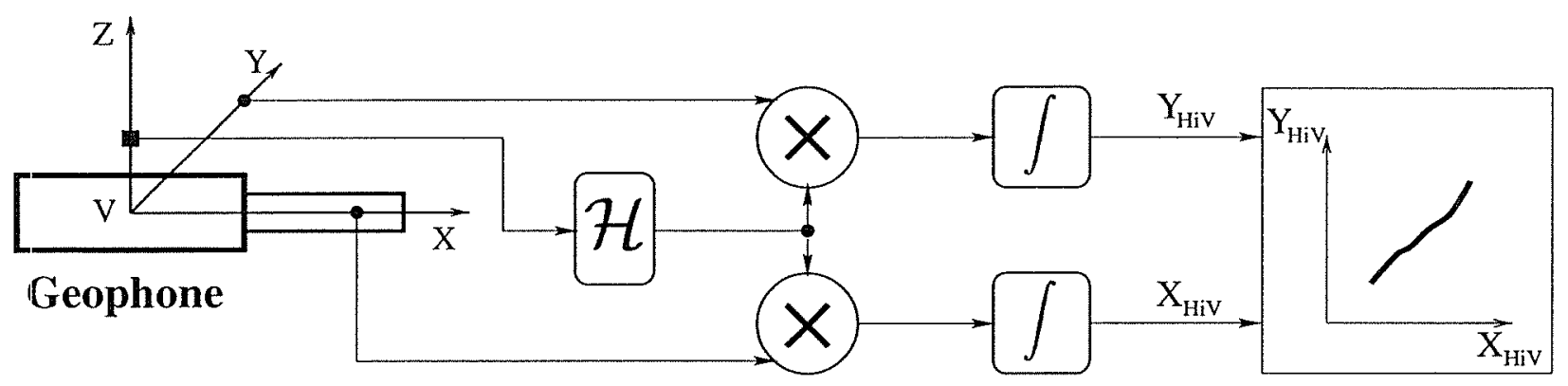

(b)

Figure 3-4: Schemes of processing in HV/HiV MPD methods: (a) HV MPD method; (b) HiV MPD method: the block with $\mathcal{H}$ in it corresponds to the operation of phase shifting vertical component for $\pi / 2$ radians which is achieved using Hilbert transform.

processor (name suggested by J.E. White in [53]) will approximate the straight line passing through the origin and randomly scattered points otherwise. The straight line in case of successful detection will determine the bearing of the source, because we consider here only vertically polarized waves. Recall that the polarization plane is the vertical plane that contains both the receiver and the source of the ice event (see Fig. 3-2). As a result, this line gives also the projection of bearing of the source on the horizontal plane. Using the output of such a processor for several geophones we can easily triangulate the source location in the horizontal plane. 


\subsubsection{Procedure for isolating elliptic polarization in data record- ings by the MPD method}

A similar idea is employed in the HiV processor ${ }^{1}$ (see Fig. 3-4b). The main difference between $\mathrm{HV}$ and $\mathrm{HiV}$ processors is that in the latter the vertical component is shifted in phase by $\pi / 2$ radians. Originally, I used numerical differentiation to achieve this phase shift, but due to the nonuniform frequency response of the numerical differentiation operation, I later switched to the Hilbert transform to achieve that purpose. The result of averaging the product of the horizontal $X$-component with the inverted vertical $Z$-component is called $X_{\mathrm{Hiv}}$, while the result for the horizontal $Y$-component is called $Y_{\mathrm{Hiv}}$. We can express them at the time $t$ as:

$$
\begin{gathered}
X_{\mathrm{HV}}(t)=\int_{t-T_{I} / 2}^{t+T_{I} / 2} v_{x} v_{z} \mathrm{~d} t \\
Y_{\mathrm{HV}}(t)=\int_{t-T_{I} / 2}^{t+T_{I} / 2} v_{y} v_{z} \mathrm{~d} t \\
X_{\mathrm{HiV}}(t)=\int_{t-T_{I} / 2}^{t+T_{I} / 2} v_{x} v_{z}^{\pi / 2} \mathrm{~d} t \\
Y_{\mathrm{HiV}}(t)=\int_{t-T_{I} / 2} v_{y} v_{z}^{\pi / 2} \mathrm{~d} t
\end{gathered}
$$

where $v_{x}, v_{y}, v_{z}$ are the corresponding components of the velocity, $v_{z}^{\pi / 2}$ is the phaseshifted vertical component, $T_{I}$ is the integration (or averaging) interval. For discrete

\footnotetext{
${ }^{1} \mathrm{HiV}$ according to [53] stands for Horizontal and inverted Vertical components.
} 
signals the above relations transform to

$$
\begin{aligned}
X_{\mathrm{HV}}\left(t_{j}\right) & =\sum_{i=-M / 2}^{M / 2} v_{x}\left(t_{j}+i \Delta t\right) v_{z}\left(t_{j}+i \Delta t\right) \\
Y_{\mathrm{HV}}\left(t_{j}\right) & =\sum_{i=-M / 2}^{M / 2} v_{y}\left(t_{j}+i \Delta t\right) v_{z}\left(t_{j}+i \Delta t\right) \\
X_{\mathrm{HiV}}(t) & =\sum_{i=-M / 2}^{M / 2} v_{x}\left(t_{j}+i \Delta t\right) v_{z}^{\pi / 2}\left(t_{j}+i \Delta t\right) \\
Y_{\mathrm{HiV}}(t) & =\sum_{i=-M / 2}^{M / 2} v_{y}\left(t_{j}+i \Delta t\right) v_{z}^{\pi / 2}\left(t_{j}+i \Delta t\right)
\end{aligned}
$$

To visualize the processing results, $Y_{\mathrm{HiV}}$ is usually plotted versus $X_{\mathrm{HiV}}$.

As a result at the output of such a processor we would get a greatly enhanced SV-wave (or Rayleigh wave), because the phase-shifted vertical component will now be in phase with horizontal components. The other waves will produce a substantially attenuated output due to the different phase relationship between vertical and horizontal components in those waves.

So as a result the HV MPD method can be used as compressional wave detector, while its HiV counterpart is used for SV-waves or Rayleigh waves. Especially useful is the ability of this method to achieve simultaneously both the separation of the different polarizations of seismic waves and the bearing of the source of the ice event.

\subsection{Data processing scheme}

Using the MPD polarization processing method the development of ice fractures in the array near-field was successfully tracked in the time and spatial domains.

The visualization procedure is a little bit different from the one described for both processors. The reason for this change is the presence of two coordinate systems in this particular experimental setup: the first one (further referred to as $\mathbb{S}_{a}$ ) is the Cartesian coordinate system in which the locations of sensors (geophones) are defined, 


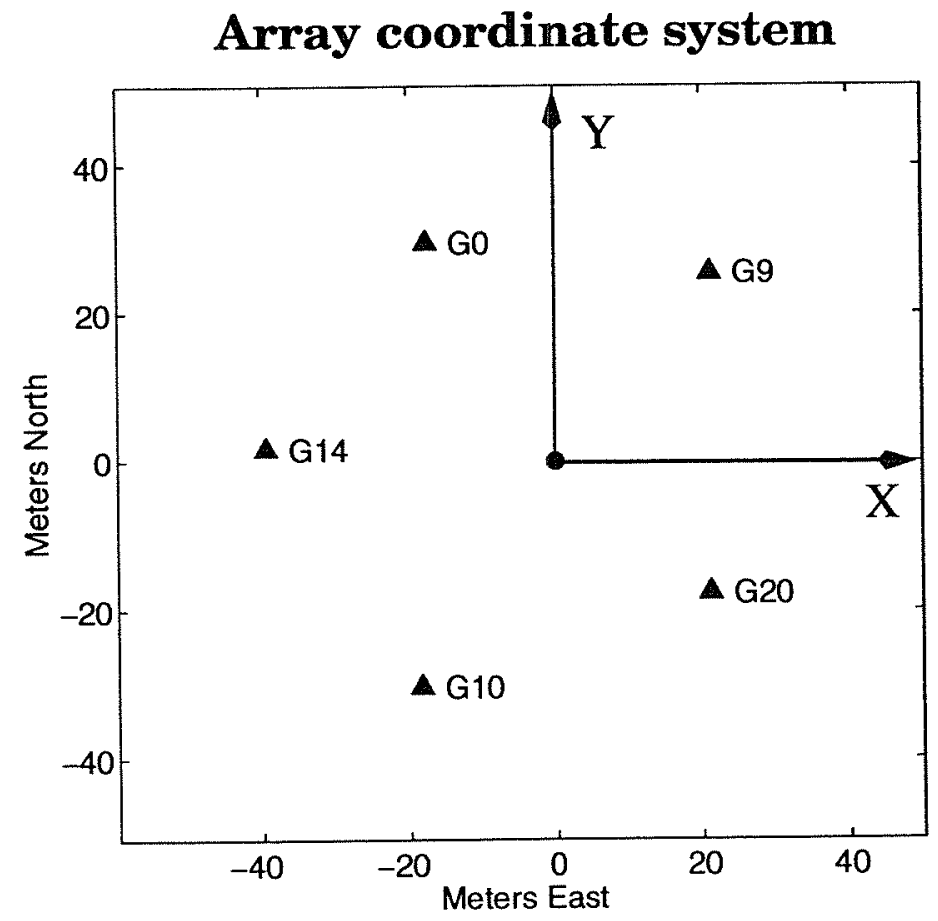

\section{Geophone coordinate system}

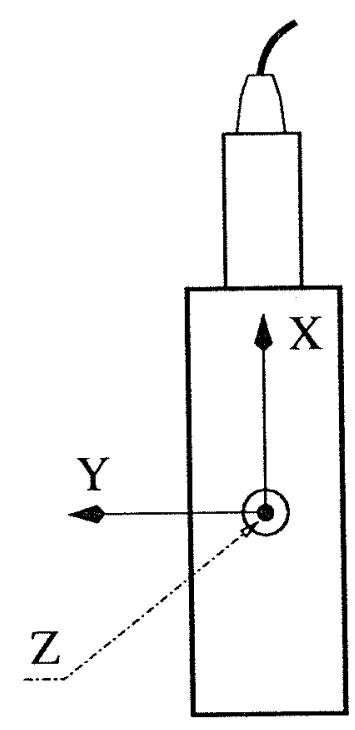

Figure 3-5: Two coordinate systems relevant to the geophone data. The top plot shows the coordinate system of the geophone array (referred to as $\mathbb{S}_{a}$ in the text). Markers show the locations of geophones. The origin is usually the location of the RLAM antenna. The direction of the $x$-axis is due East, that of the $y$-axis is due North. The bottom plot shows the plan view of a geophone with its internal coordinate system (this system is called $\mathbb{S}_{g}$ in the text). The direction of the $x$-axis (main geophone axis) is due North, that of the $y$-axis is due West. These directions are identical for all geophones due to the "conceptual" rotation procedure. 


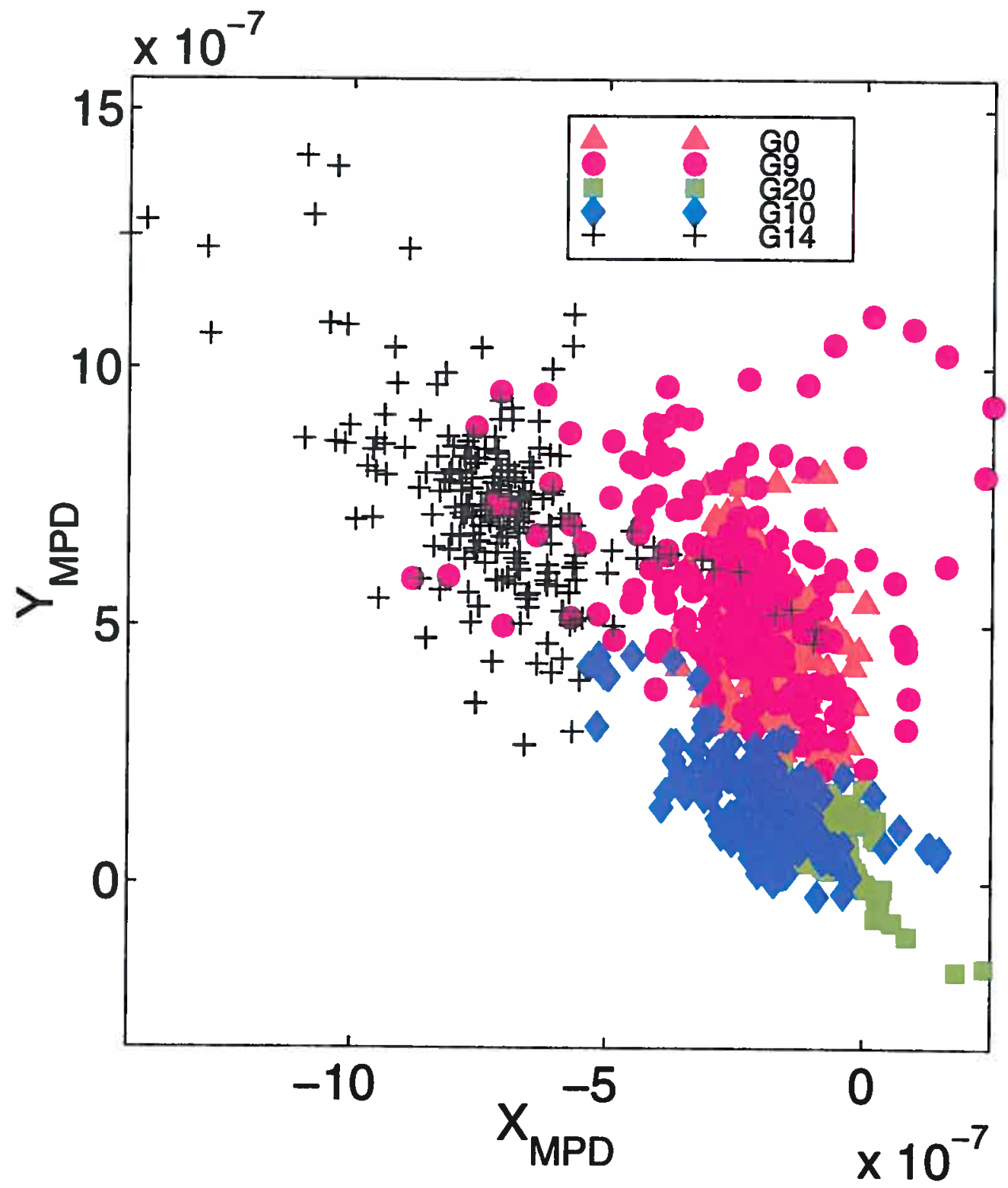

Figure 3-6: Processing results of the Event F5-3 (see in the text) with the HV MPD method. For definitions of $X_{\mathrm{MPD}}$ and $Y_{\mathrm{MPD}}$ see Equations (3.5) and (3.6). Different markers correspond to different geophones (see legend). For correspondence between geophone names and geophone numbers used throughout the text see Fig. 3-8. 


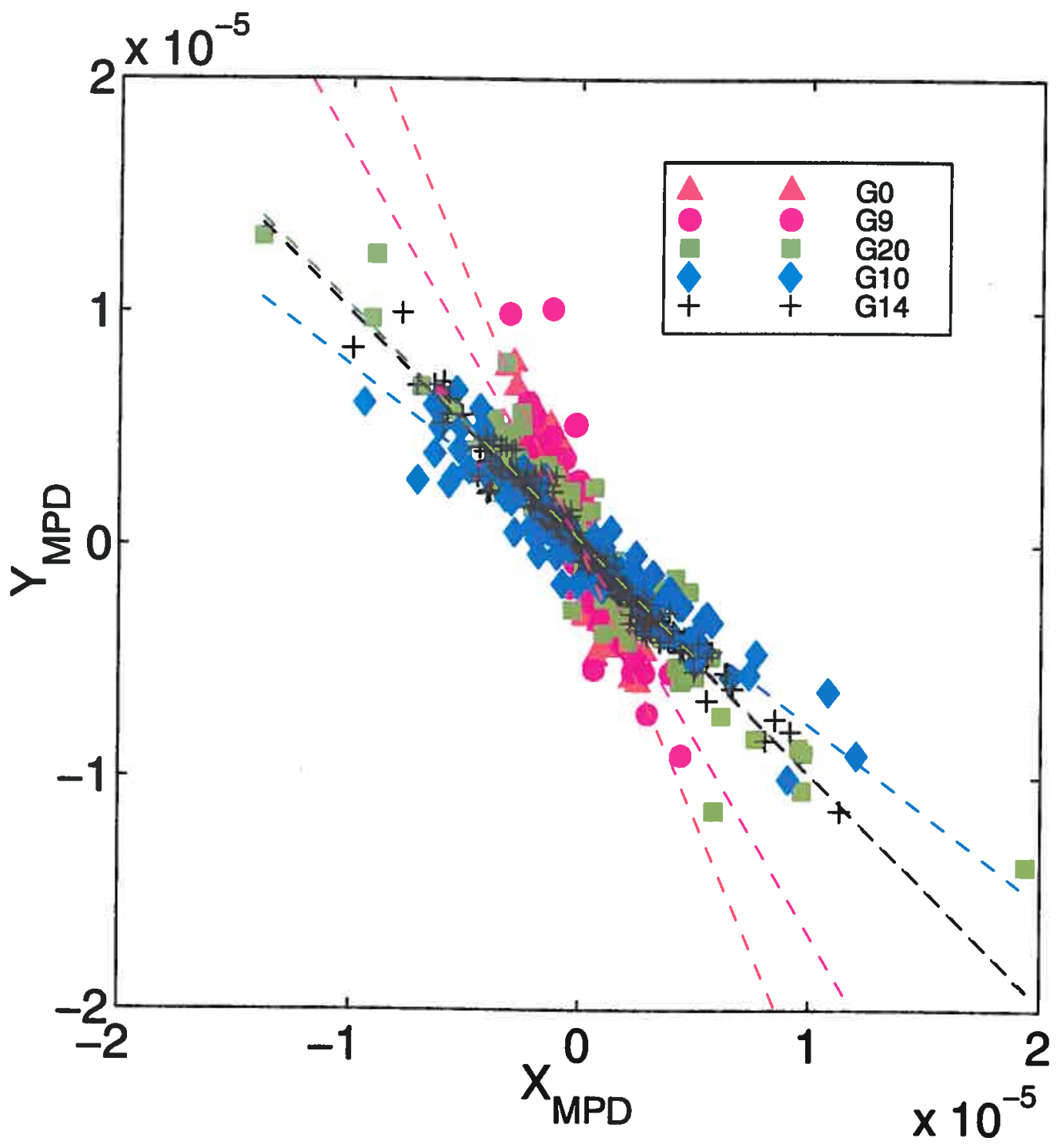

Figure 3-7: Processing results of the Event F5-3 (see in the text) with HiV MPD method. For definitions of $X_{\mathrm{MPD}}$ and $Y_{\mathrm{MPD}}$ see Equations (3.7) and (3.8). Different markers correspond to different geophones (see legend). The best least-square fit lines for different geophones are shown by color-coded (the color of the line is the same as a color of the marker corresponding to the respective geophone) dashed lines. For correspondence between geophone names and geophone numbers used throughout the text see Fig. 3-8. 


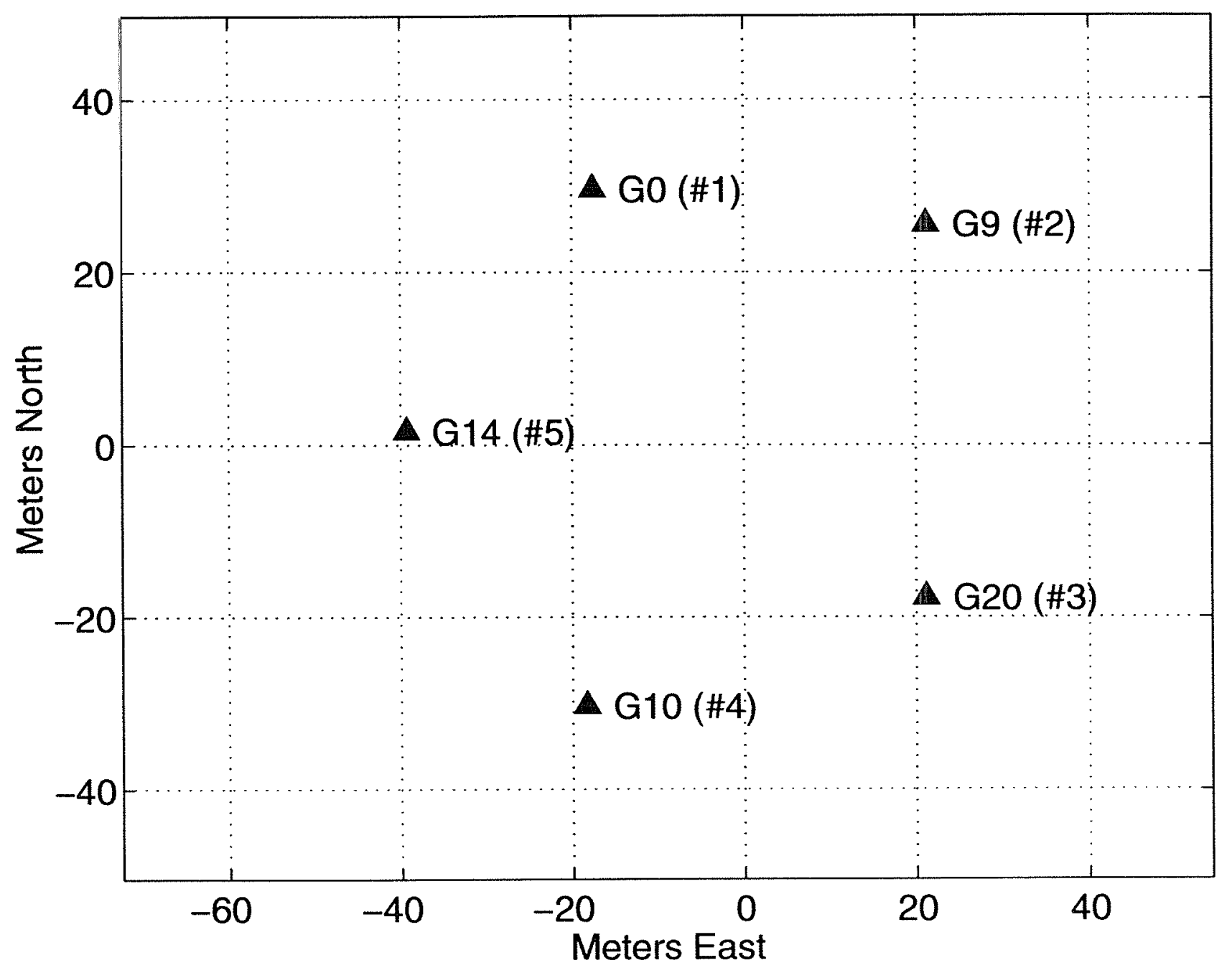

Figure 3-8: Geophone array layout. The geophones are shown by filled triangles with geophone names written near them. Numbers in parenthesises are used to reference geophones throughout the text. So, for example, geophone G20 is referred to as geophone \#3 in the text. 




Figure 3-9: Processing results of the Event F5-5 (see in the text) with the HV MPD method. For definitions of $X_{\mathrm{MPD}}$ and $Y_{\mathrm{MPD}}$ see Equations (3.5) and (3.6). Different markers correspond to the results of processing of different geophones (see legend). For correspondence between geophone names and geophone numbers used throughout the text see Fig. 3-8. 


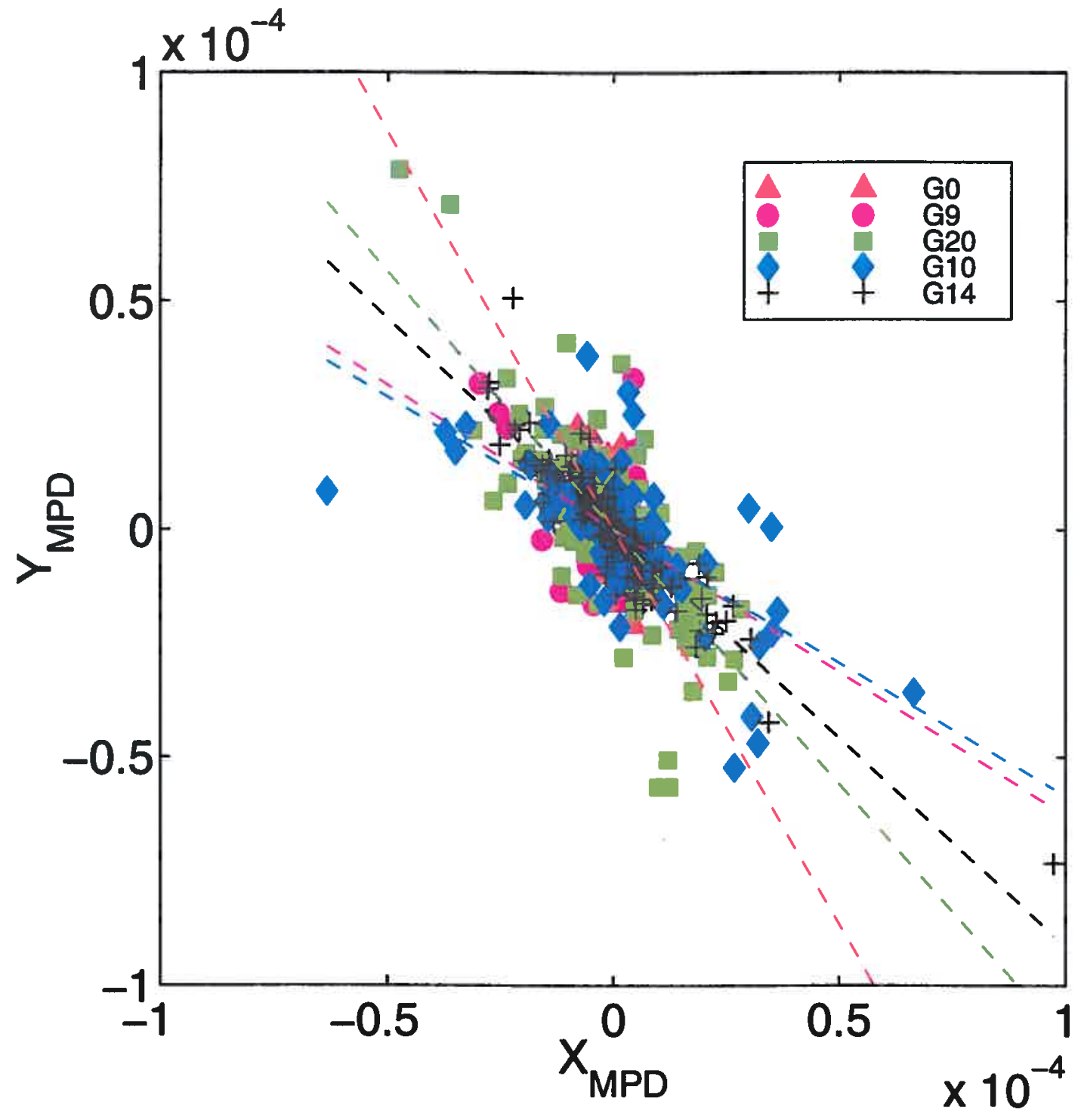

Figure 3-10: Processing results of the Event F5-5 (see in the text) with the HiV MPD method are shown. For definitions of $X_{\mathrm{MPD}}$ and $Y_{\mathrm{MPD}}$ see Equations (3.7) and (3.8). Different markers correspond to the results of processing of different geophones (see legend). The best least-square fit lines for different geophones are shown by colorcoded (the color of the line is the same as a color of the marker corresponding to the respective geophone) dashed lines. For correspondence between geophone names and geophone numbers used throughout the text see Fig. 3-8. 
the other (further referred to as $\mathbb{S}_{g}$ ) - is the internal Cartesian coordinate system of each geophone ${ }^{2}$.

For the coordinate system $\mathbb{S}_{a}$ the direction of the $x$-axis is due East and that of the $y$-axis is due North (see top plot on Fig. 3-5). For the coordinate system $\mathbb{S}_{g}$ the direction of the $x$-axis is due North and that of the $y$-axis is due West (see bottom plot on Fig. 3-5). Hence, in order to be able to use the results of the motion product detector for source location triangulation, we need to reconcile the different conventions in these two coordinate systems. For triangulation purposes it is better: to choose the convention of $\mathbb{S}_{a}$, so for the $\mathrm{HV}$ MPD processor, actually $Y_{\mathrm{MPD}}^{\mathrm{HV}}$ is plotted versus $X_{\mathrm{MPD}}^{\mathrm{HV}}$ :

$$
\begin{aligned}
& X_{\mathrm{MPD}}^{\mathrm{HV}}=-Y_{\mathrm{HV}} \\
& Y_{\mathrm{MPD}}^{\mathrm{HV}}=X_{\mathrm{HV}},
\end{aligned}
$$

where $X_{\mathrm{HV}}$ and $Y_{\mathrm{HV}}$ are defined by Equations (3.1) and (3.2), respectively.

Similarly, for the $\mathrm{HiV}$ MPD processor $Y_{\mathrm{MPD}}^{\mathrm{HiV}}$ is plotted versus $X_{\mathrm{MPD}}^{\mathrm{HiV}}$ :

$$
\begin{aligned}
X_{\mathrm{MPD}}^{\mathrm{HiV}} & =-Y_{\mathrm{HiV}} \\
Y_{\mathrm{MPD}}^{\mathrm{HiV}} & =X_{\mathrm{HiV}}
\end{aligned}
$$

where $X_{\mathrm{HiV}}$ and $Y_{\mathrm{HiV}}$ are defined by Equations (3.3) and (3.4), respectively.

\subsection{Results of data processing by the MPD method}

The processed data was collected on a small ice floe which was located approximately $4 \mathrm{~km}$ North-East of the main camp. The geophone array layout at that site and the correspondence between geophone names (used on plot legends) and geophone numbers (used throughout the text) are shown on Fig. 3-8.

Here I will consider in detail the results of the two following events:

\footnotetext{
${ }^{2}$ Due to the "conceptual" rotation procedure (see above) the orientations of the main axes of all geophones are identical within the precision of angular measurement of geophones' orientations
} 


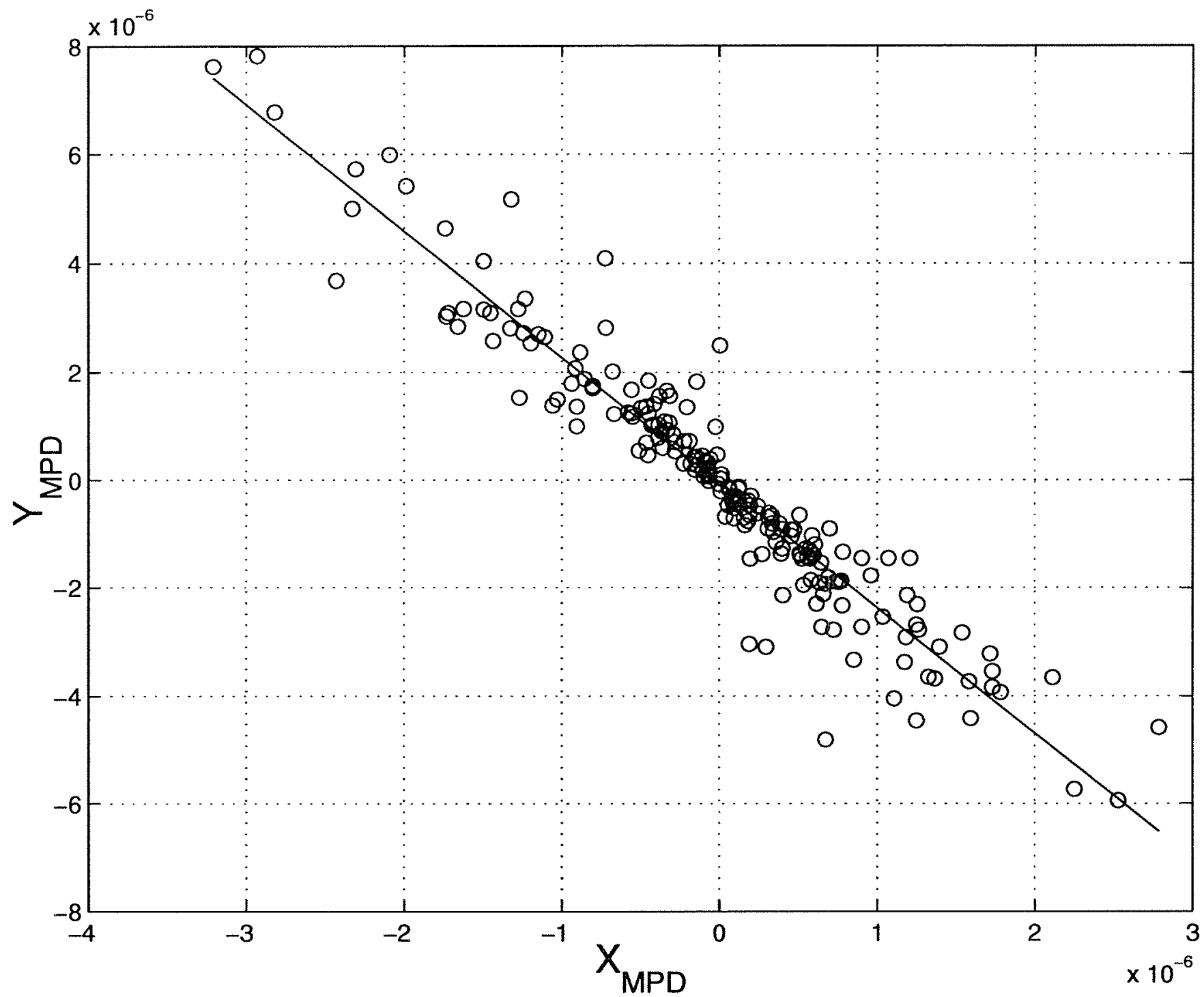

Figure 3-11: Best least-square fit line for processing results of the Event F5-3 (see in the text) with the HiV MPD method for geophone \#1 (G0). The results are shown by circle markers. The correlation coefficient for this fit is $95.4 \%$. 


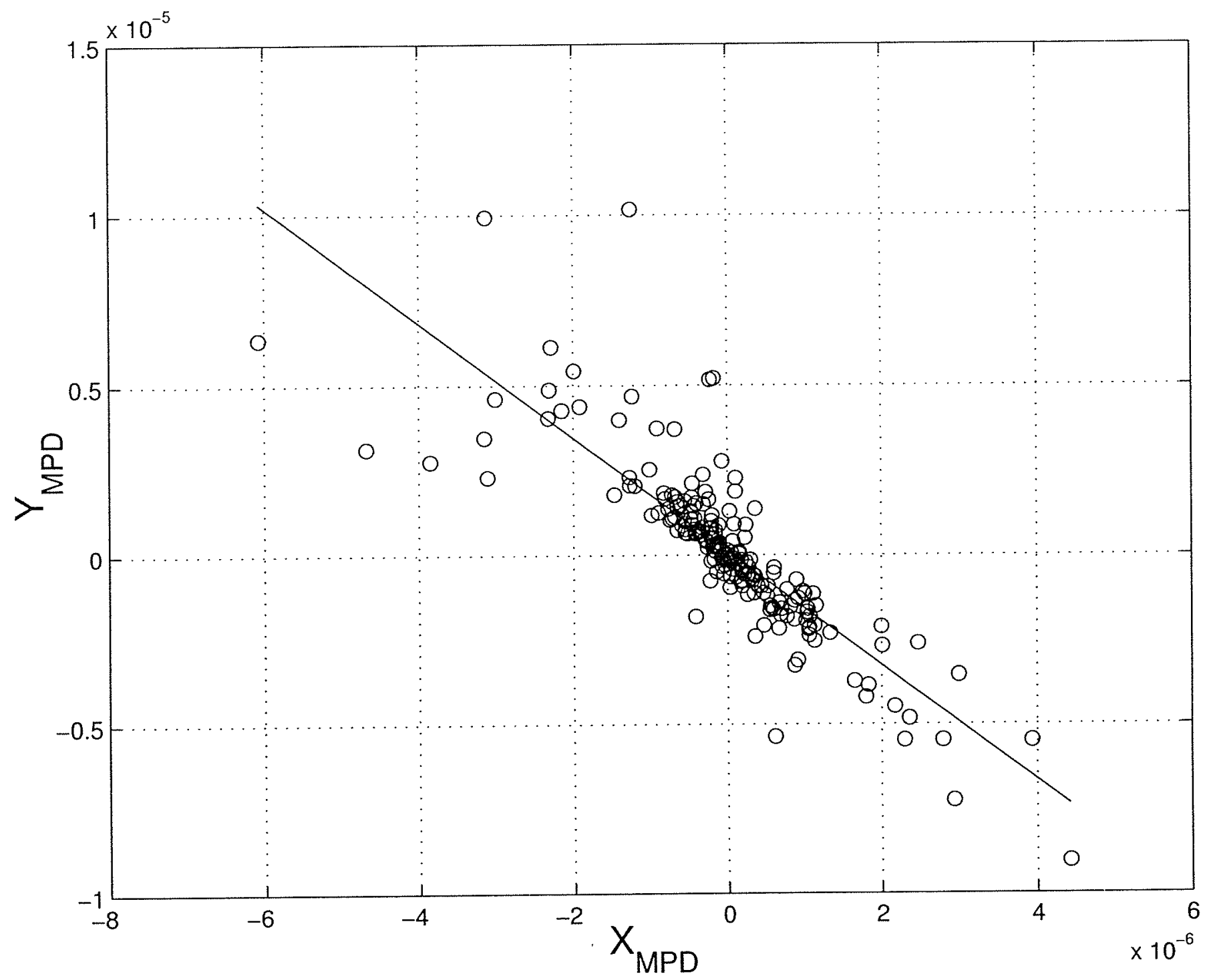

Figure 3-12: Best least-square fit line for processing results of the Event F5-3 (see in the text) with the HiV MPD method for geophone \#2 (G9). The results are shown by circle markers. The correlation coefficient for this fit is $84.2 \%$. 


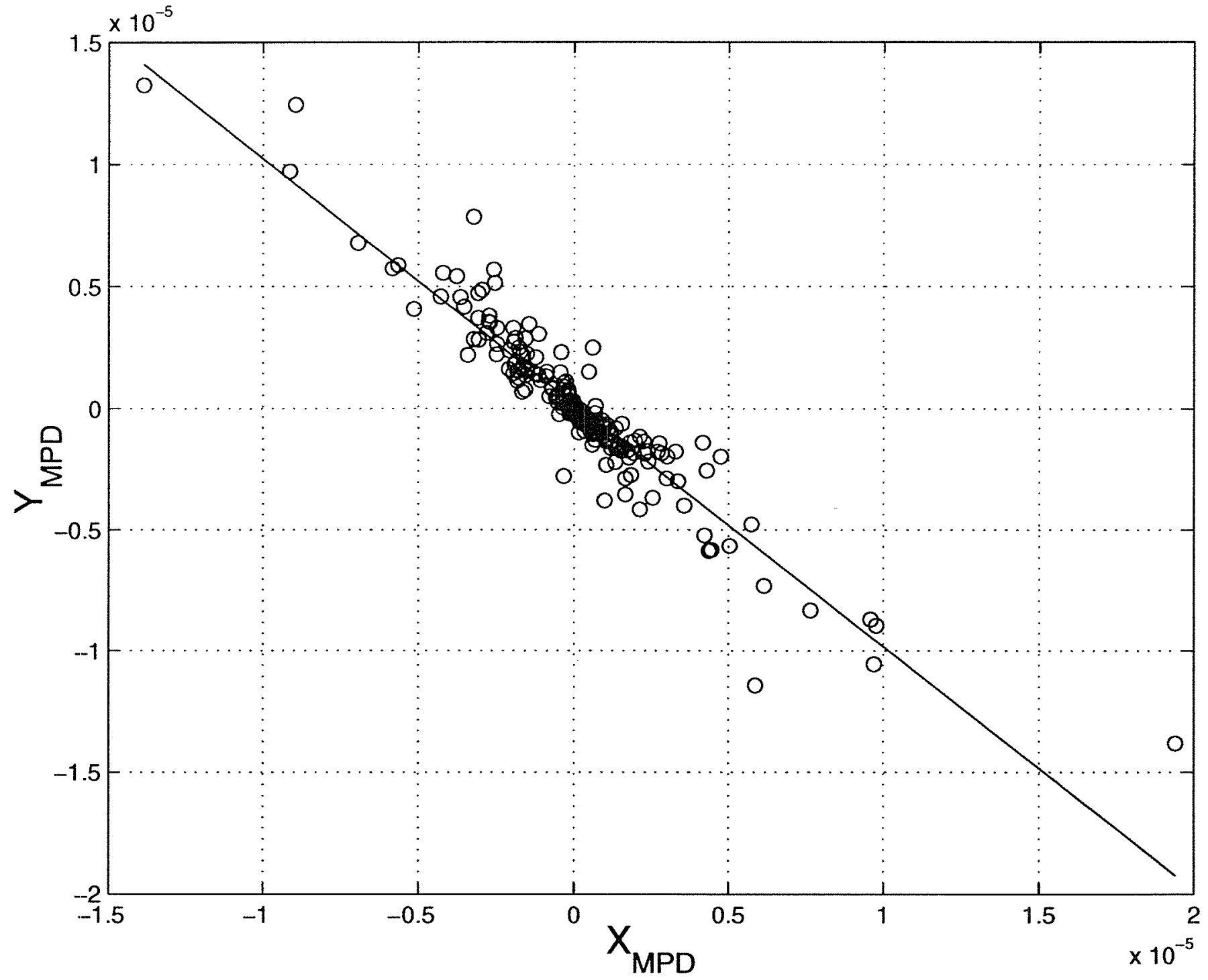

Figure 3-13: Best least-square fit line for processing results of the Event F5-3 (see in the text) with the HiV MPD method for geophone \#3 (G20). The results are shown by circle markers. The correlation coefficient for this fit is $94.3 \%$. 


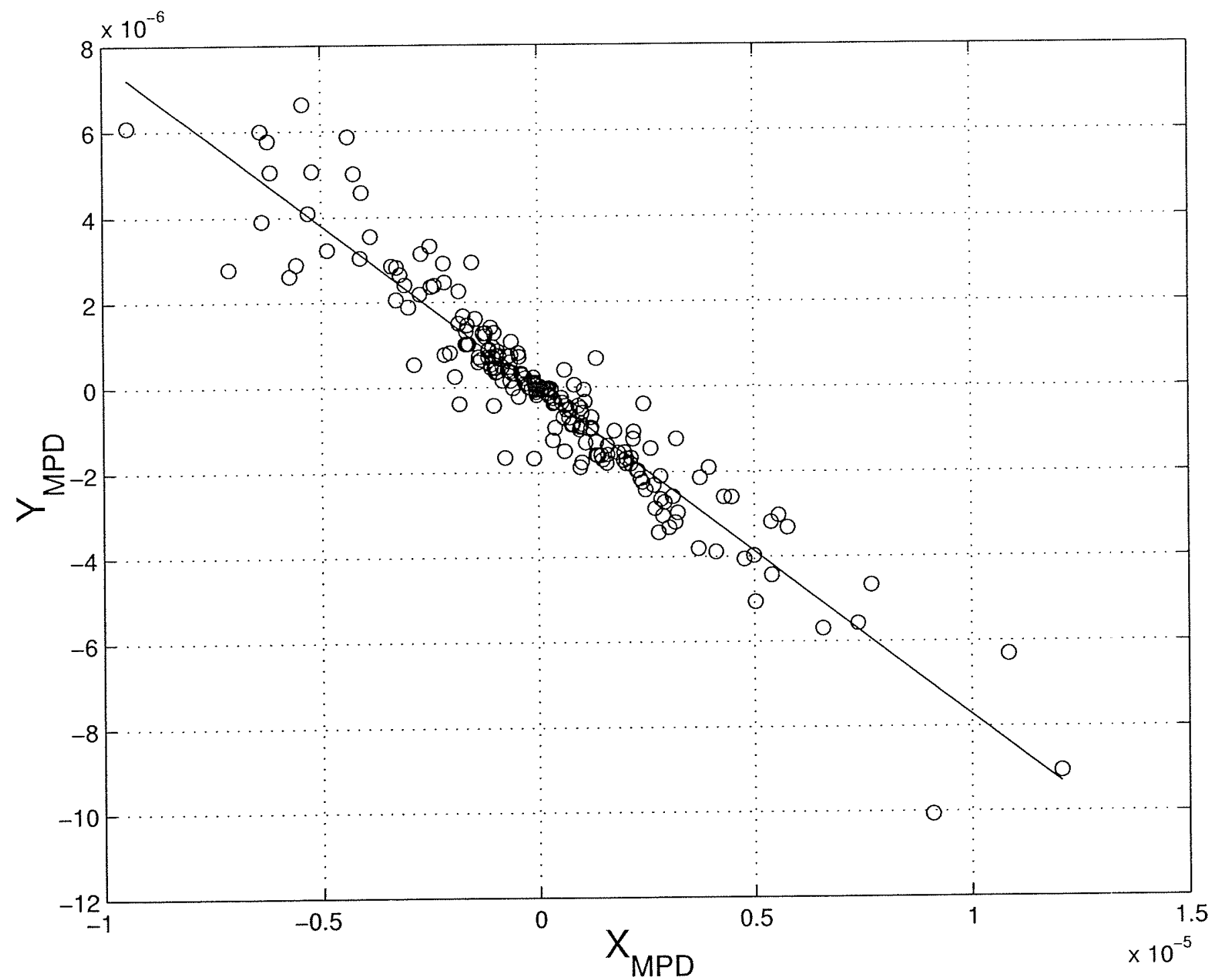

Figure 3-14: Best least-square fit line for processing results of the Event F5-3 (see in the text) with the HiV MPD method for geophone \#4 (G10). The results are shown by circle markers. The correlation coefficient for this fit is $94.8 \%$. 


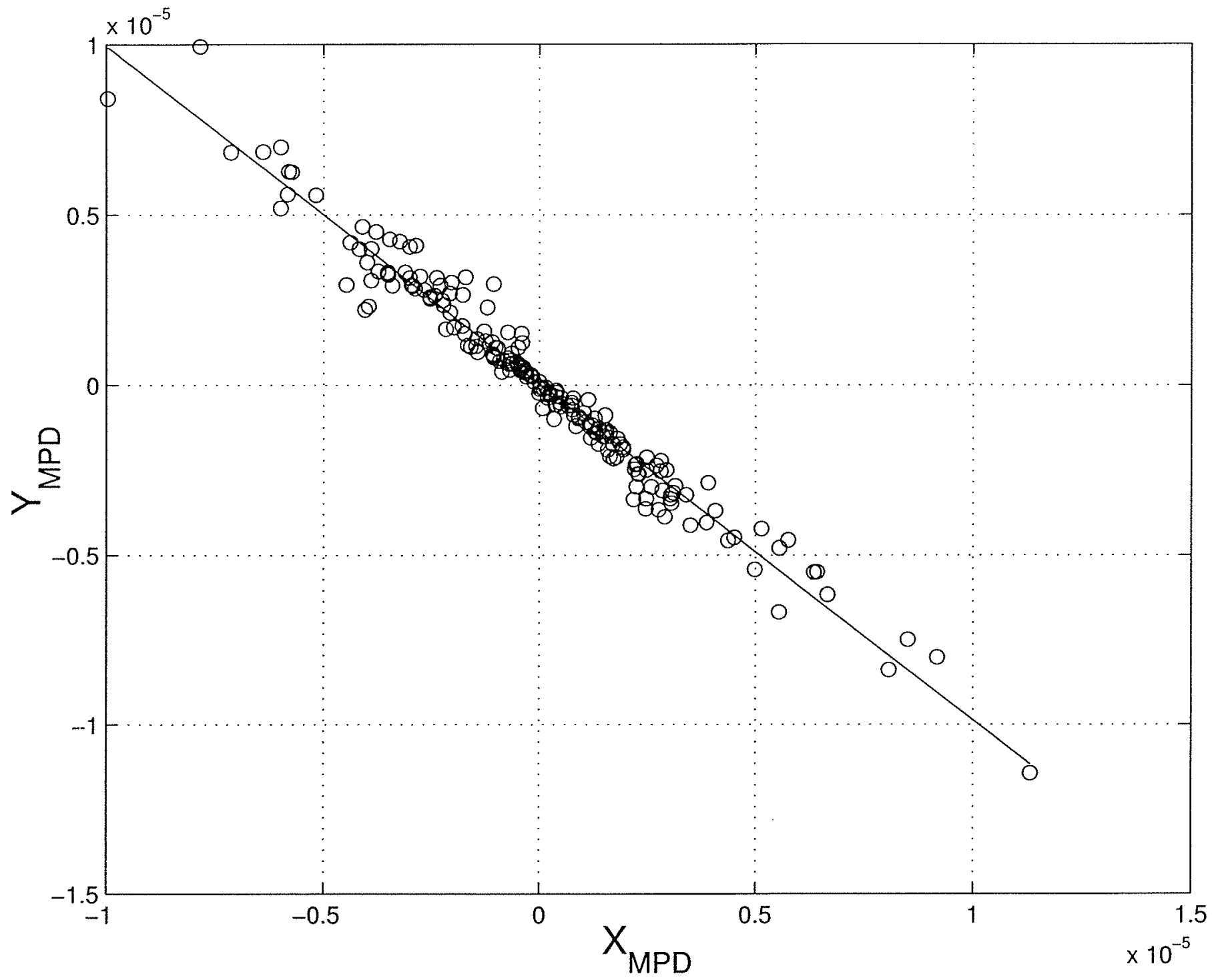

Figure 3-15: The best least-square fit line for processing results of the Event F5-3 (see in the text) with the HiV MPD method for geophone \#5 (G14). The results are shown by circle markers. The correlation coefficient for this fit is $98.4 \%$. 
1. This event occured approximately 325 sec. after the beginning of file \#5 on the RLAM tape \#26 (further referred to as RLAM-26). This event will be further referred to as Event F5-3.

2. The second event occured approximately $390 \mathrm{sec}$. after the beginning of RLAM26. This event will be further referred to as Event F5-5.

\subsubsection{MPD seismograms}

On the Fig. 3-6 the processing results of the Event F5-3 with the HV MPD method are shown (recall that in this processor there is no phase shift between the horizontal and vertical components). The different kinds of markers correspond to different geophones. On this plot we see points scattered all over the place: there is no obvious linear dependence between them. That means that no wave with strong rectilinear polarization is present in the data. On the Fig. 3-7 the processing results for the same event with the HiV MPD method are shown (recall that in this processor there is a phase shift of $\pi / 2$ between the horizontal and vertical components). Again, the different kinds of markers correspond to the results for different geophones. The results are strikingly different from that presented on Fig. 3-6. Really, we have quite evident linear dependence in the output for each geophone. The best least-square fit lines for different geophones are shown by color-coded (the color of the line is the same as a color of the marker corresponding to the respective geophone) dashed lines. These best fit lines are also shown on separate plots for each geophone: see Figs. 3-11, $3-12,3-13,3-14$, and 3-15. The correlation coefficients for those fits are quite high (usually, above 90\%). Note, that lowest correlations correspond to two geophones (\#2 (G9) and \#3 (G20)) that give quite contradictory localization results when compared to other three geophones, although the actual difference in correlation coefficients is not really significant (for example, 94.3\% - for geophone \#3 (G20) and 94.8\% - for geophone \#4 (G10)).

A similar conclusion could be reached by looking at Figs. 3-9 and 3-10 which show processing results for the Event F5-5 by the HV MPD and HiV MPD meth- 
ods respectively. Note that these two events are separated by at least $1 \mathrm{~min}$ in time, but still the similarities between them are quite evident.

\subsubsection{Triangulation results}

Fig. 3-16 shows the best fit lines drawn from corresponding geophone locations for the Event F5-3 processed by the HiV MPD method. On this plot the source location could be found by successful triangulation with at least 3 bearings from geophones (the result of such triangulation is shown by filled circle on Fig. 3-16). For the other two geophones (note that for these two geophones linear fits show lower correlation coefficients than that for other three geophones) bearings are quite contradictory to the triangulated source location. A similar plot for the Event F5-5 (see Fig. 3-17) shows identical behavior: again geophones \#1 (G0), \#4 (G10) and \#5 (G14) give good triangulation for the source, while geophones \#2 (G9) and \#3 (G20) contradict them. A possible explanation for the difference in the behavior of these two geophone groups is the hypothesis that the ice plate could have been broken somewhere between these two groups and as a result geophones \#2 and \#3 might have been on a different ice plate than the other three geophones. The horizontal refraction on the hypothetical boundary of two plates could account for differences in apparent locations of ice events for these two geophone groups.

The above procedure has been applied to several ice events. One result of this polarization analysis was that these fractures seemed to generate vertically polarized shear (SV) waves for the most part. The source distances from the array center for these events are shown versus time on Fig. 3-18. The analysis of this figure indicates that stresses redistribute and move away from the corner of two ridges with time.

On Fig. 3-19 the same events are shown overlayed on the aerial photograph of the experimental site. On this figure we can see that events mostly concentrate in the vicinity of the corner of two ice ridges. This indicates the area of high stress. Also the character of particle motion in the corresponding arrivals supplies additional evidence for the continuing ridge building process in that part of the ice island, because ice breaking during ridge building is characterized by predominantly vertical particle 


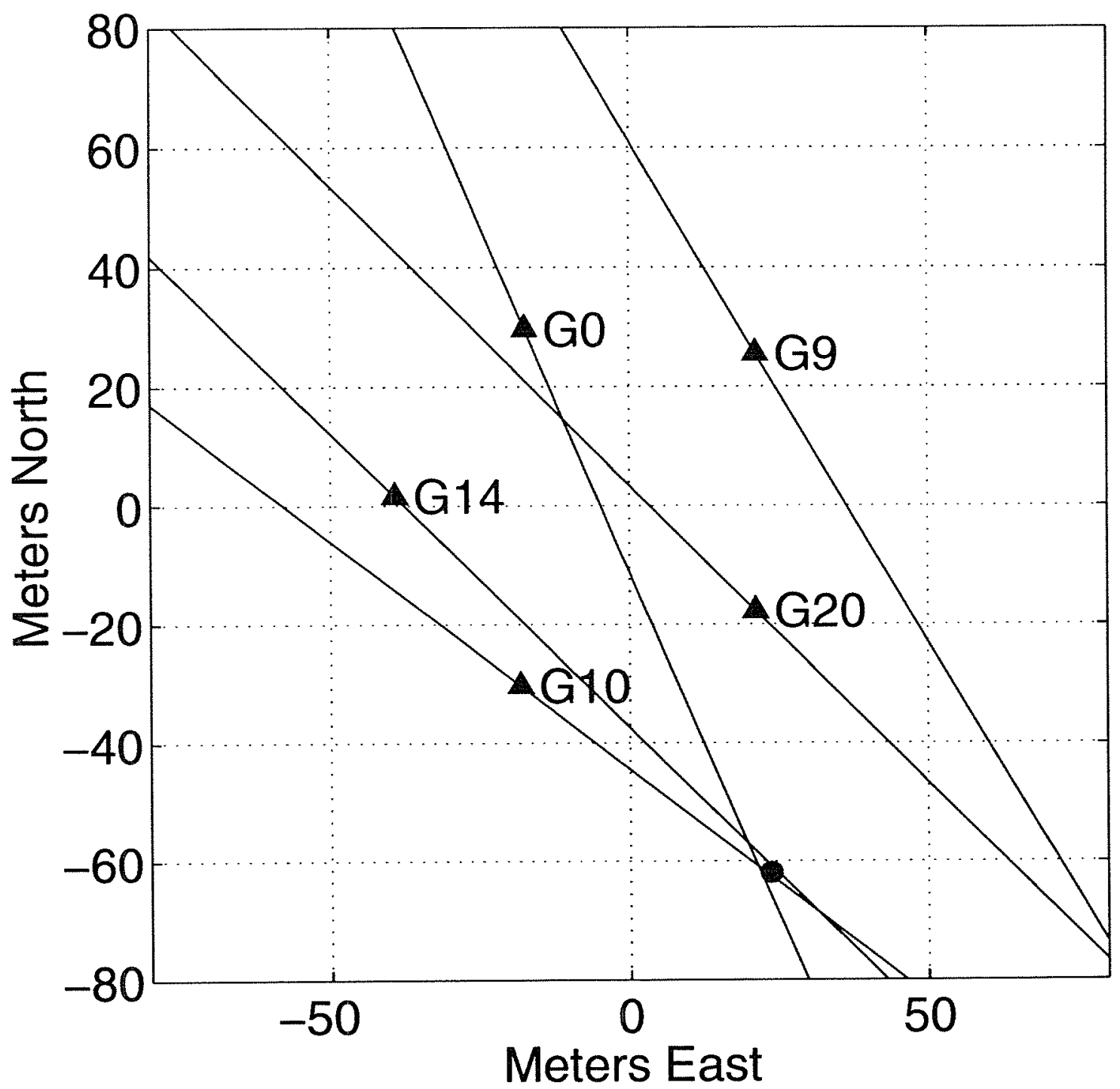

Figure 3-16: Results of the triangulation of the source location by best least-square fit lines for the Event F5-3 processed by the HiV MPD method. The triangulated source location is shown by a filled circle. The geophones \#1 (G0), \#4 (G10) and \#5 (G14) produce consistent results, while the geophones \#2 (G9) and \#3 (G20) give quite different results. 


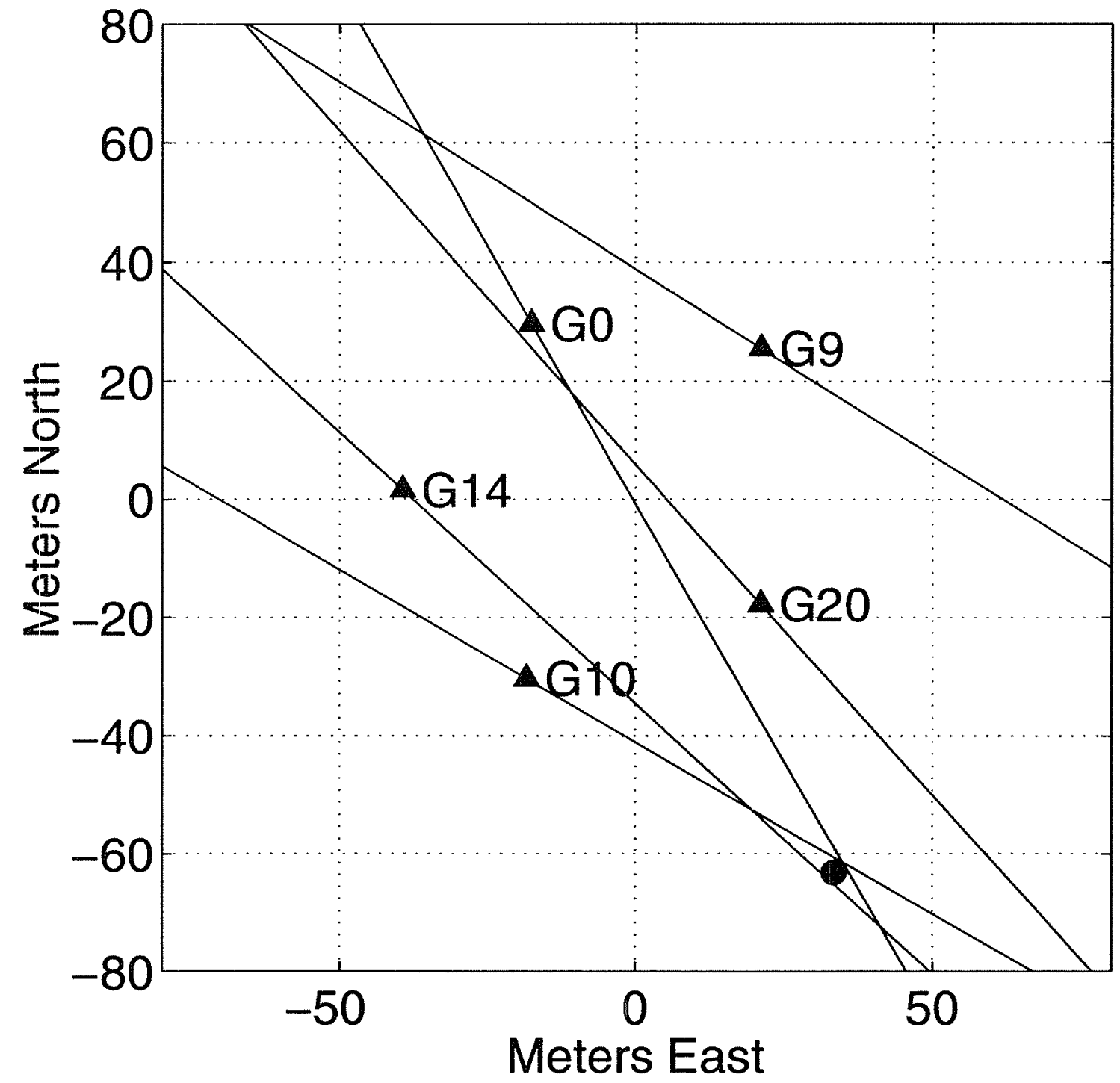

Figure 3-17: The results of the triangulation of the source location by best least-square fit lines for the Event F5-5 processed by the HiV MPD method. The triangulated source location is shown by a filled circle. Again, like for the Event F5-3 the geophones \#1 (G0), \#4 (G10) and \#5 (G14) produce consistent results, while the geophones \#2 (G9) and \#3 (G20) give quite different results (compare with Fig. 3-16). 


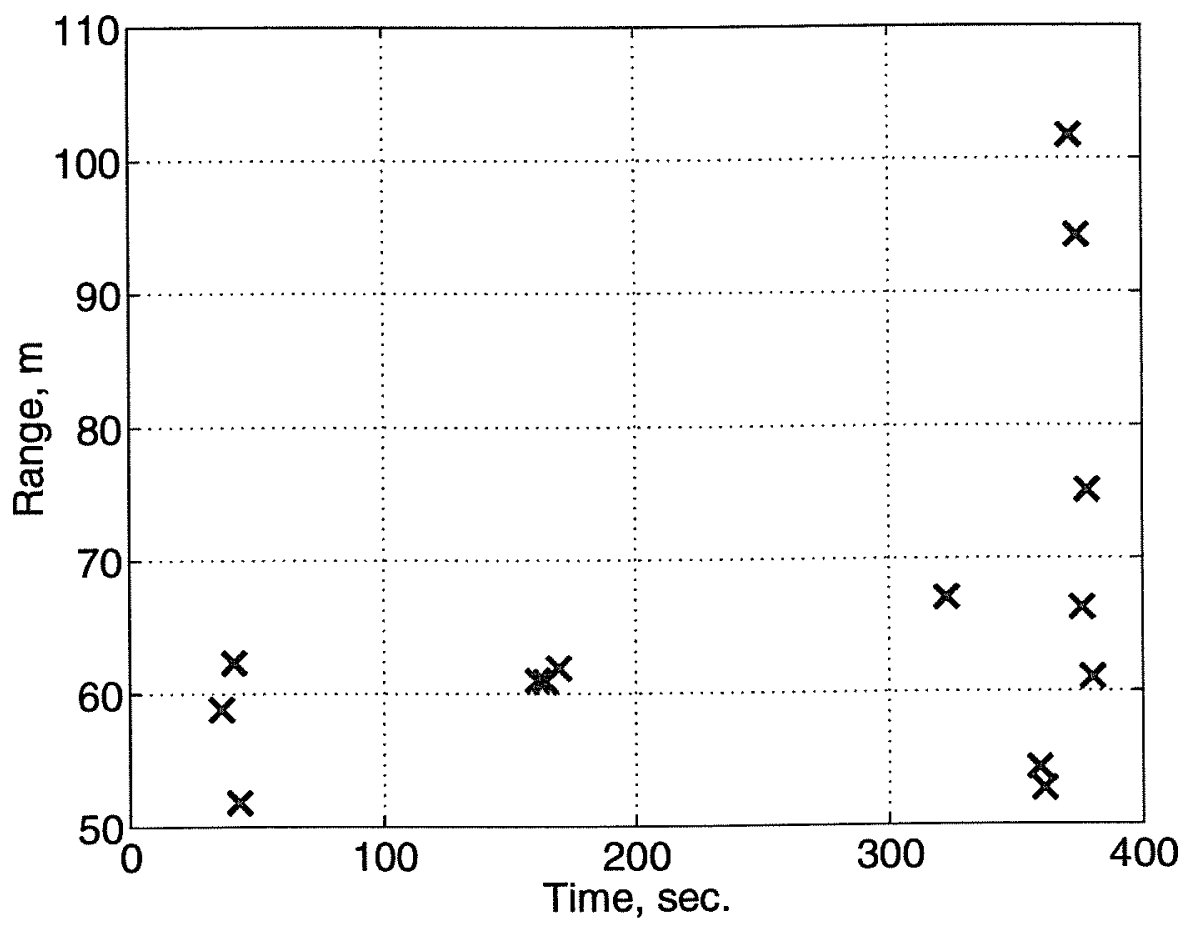

Figure 3-18: Ranges from array apex versus time for events located by triangulation using HiV MPD processing results.

motion as opposed to the horizontal particle motion in compressional waves. The stress relief is most probably occurring in the ice lead which could be identified on the figure by the location of hydrophone deployment.

\subsection{Conclusions}

Polarization methods are well known in seismology, but they have never been used for ice event data processing. In this chapter one of the polarization methods (so called Motion Product Detector method) has been successfully applied for the localization of ice events recorded by the RLAM box deployed on "ice island", which is a remote experimental site, approximately $4 \mathrm{~km}$ East of the base camp, and the determination of the polarization characteristics of the elastic waves generated by these events. The fractures identified by this method seemed to generate vertically polarized shear (SV) waves for the most part. Their concentration in the vicinity of the corner of two ice ridges and the character of particle motion in the corresponding arrivals indicate the 


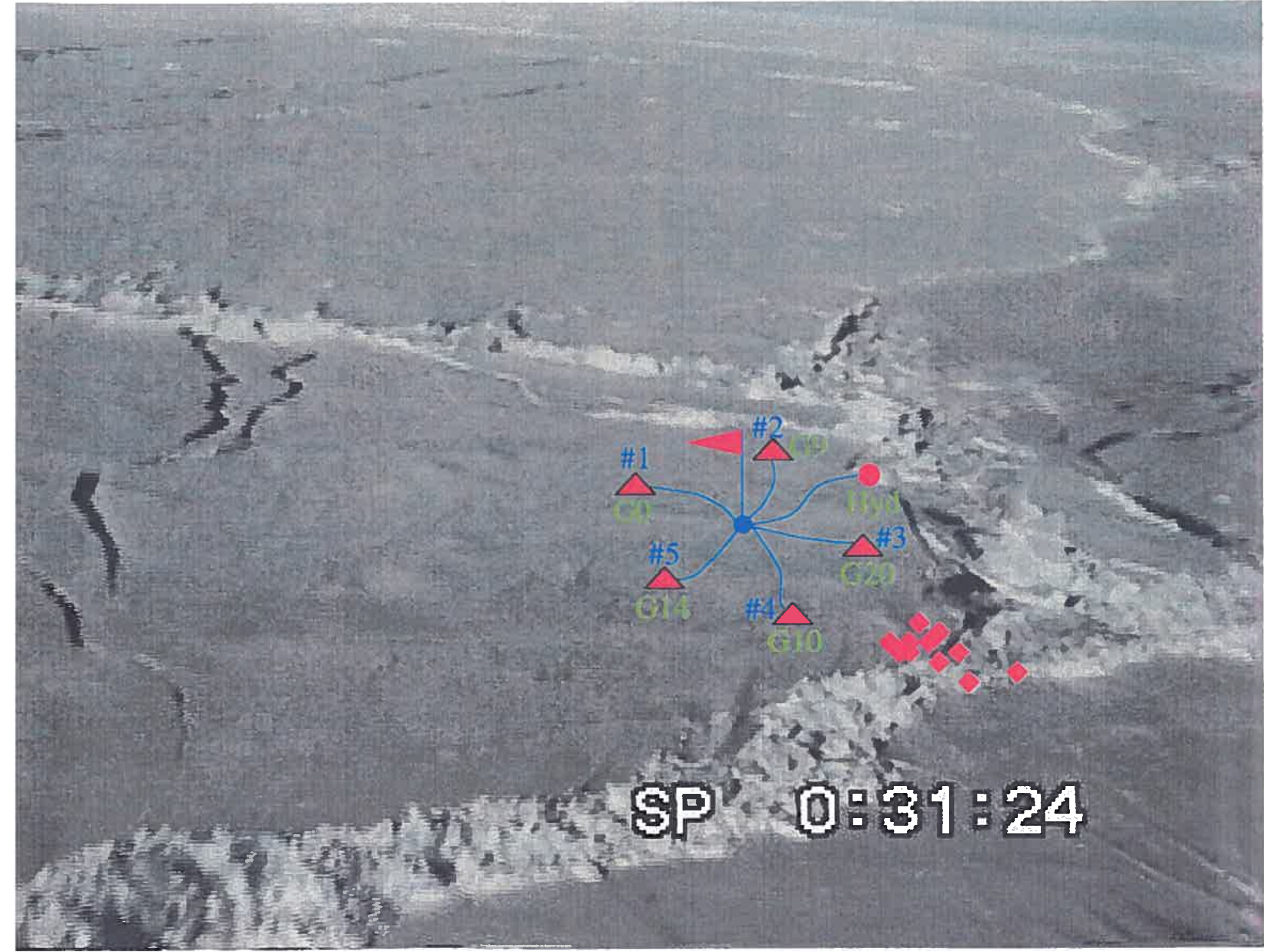

$\triangle$ 3-axis geophone

RLAM antenna

- Hydrophone

- Event location

Figure 3-19: Event locations determined by triangulation using HiV MPD processing results overlayed on the aerial photo of the "ice island". On this figure the geophone locations are shown by filled triangles with geophone names and reference numbers written next to markers. The location of hydrophone is shown by filled circle. 
continuing ridge building process in that part of the ice island.

This application demonstrated the feasibility of the polarization method for ice event data processing, because it allows to identify areas of high stress concentration and "hot spots" in ridge building process. Furthermore, the polarization characteristics of elastic waves could be used for establishing the physics of the processes in the ice sheet which lead to generation of ice events. 


\section{Chapter 4}

\section{Edge waves}

\subsection{Overview}

In several ice events recorded on the so-called "ice river" site, the site near the ice lead approximately $2 \mathrm{~km}$ north of the base camp, evidence was found for the existence of so-called 'edge waves': waves propagating along the edges of a newly opened lead.

The existence of similar 'edge wave' phenomena have been reported in the literature. Recently, Goldstein et al. [14] demonstrated theoretically the existence of a new type of waves in the water/ice system containing a rectilinear crack extending through the ice cover. They found that edge waves were propagating in the water along the crack and inducing the ice cover deformation. The role of the ice sheet in their study was to modify the boundary conditions at the upper boundary of the fluid, i.e. the ice was only involved as an external loading. This loading (mainly stiffness, because they considered an inertia-less thin plate model of the ice sheet) modified the dispersion relation for the gravity surface waves on the sea surface. The crack considered was essentially a line of contact between two ice plates which were not interacting in bending. Goldstein's solution describes the diffraction of the waterborn bending-gravitational waves at the infinitely thin ice crack. However, Goldstein's approach does not directly apply to the SIMI-94 experimental observations: first, by using the model for the ice sheet as an inertia-less thin plate it discards all types of elastic waves that propagate in the ice sheet; further, the model of the crack as having 
zero width certainly can not be applied to the ice lead.

An extensive literature exists on waves in plates without fluid loading, with some papers devoted to 'edge waves' phenomena. Shaw [47] found experimental evidence for 'edge waves' in thick barium titanate disks. He investigated their resonant properties with the objective of using them in transducers. He discovered that, as the frequency approached a certain value, depending on the disk thickness, the disk vibration was consistent with a surface wave resonance with maximum motion occurring at the edge of the disk. He also found that this resonance was formed by several modes of the elastic disk.

An attempt to describe this experimental data theoretically was first made by Gazis and Mindlin [13]. They set up approximate equations of extensional motion of an elastic plate using a modal expansion in terms of a finite number of the modes of an infinite plate. Their theory was limited to axially symmetric vibrations of a circular disk. They showed that the 'edge mode' in this case arises from the complex conjugate roots of the frequency equation for the modes of the infinite plate. They investigated the properties of the 'edge mode' in the study of the reflection of straightcrest extensional waves at the edge of a semi-infinite plate. In this geometry the 'edge mode' was revealed by the substantially increased amplitudes (up to 7.7 times the amplitude of the incident wave) of the reflected complex waves. Nevertheless, Gazis and Mindlin did not achieve quantitative agreement with the experimental data obtained by Shaw. As shown later by Torvik [51], the main reason was that Gazis and Mindlin used an approximation of the frequency spectrum rather than exact roots of the Rayleigh-Lamb equation, and too few modes were employed in the model. Torvik improved the model by using the exact frequency spectrum and by using many more modes, and a greatly improved agreement with Shaw's experiment was achieved.

The more recent work by Gregory and Gladwell [15] used a 'method of projections' developed by the authors for solving similar problems in elastostatics. They represented the stress function as a sum of some inhomogeneous term and series of orthogonal functions. Their approximate solution was the best approximation in the 
least-square sense, and they again confirmed the existence of the 'edge mode' at a single isolated value of the frequency by considering the normal incidence of the plainstrain symmetric Rayleigh-Lamb wave onto the free edge of the semi-infinite plate. They found that the resonance peak was much narrower and higher than previously found by Torvik, and Gazis and Mindlin. However, the peak was bounded, indicating energy dissipation in the near-edge region.

A common feature of all of this past work is the resonant nature of the 'edge mode', appearing at discrete frequencies. Also, the past theoretical work considered normal incidence waves on the free edge. This is significantly different from what was observed during SIMI-94: the waves observed along the lead were of true broadband nature, and similar in behavior to normal seismic waves in the ice sheet, but with a characteristic periodicity and propagation direction parallel to the lead. The reason for the existence of "edge mode" is that a flexural wave propagating along a stressrelease ice edge will be slower (the plate is softer at the edge) than a flexural wave in the infinite plate. This speed gradient near the edge provides a waveguide.

The problem in the theoretical treatment of the problem is the free edge of the ice plate which makes the problem non-separable. Therefore, in contrast to the infinite plate problem [41], [39], a simple spectral integral solution cannot be derived.

These circumstances led to the construction of an approximate model which could explain the characteristics and behavior of the observed edge waves. The model was based on the lowest order modes of the infinite plate, coupled through integral boundary conditions at the free edge.

This chapter first describes the experimental setup, followed by the processing schemes applied. Then the approximate theoretical model is described, and the comparison with the experimental data is discussed. 
17 Apr. 1994

$07.11 \mathrm{Z}$

18 Apr. 1994

$21.48 \mathrm{Z}$
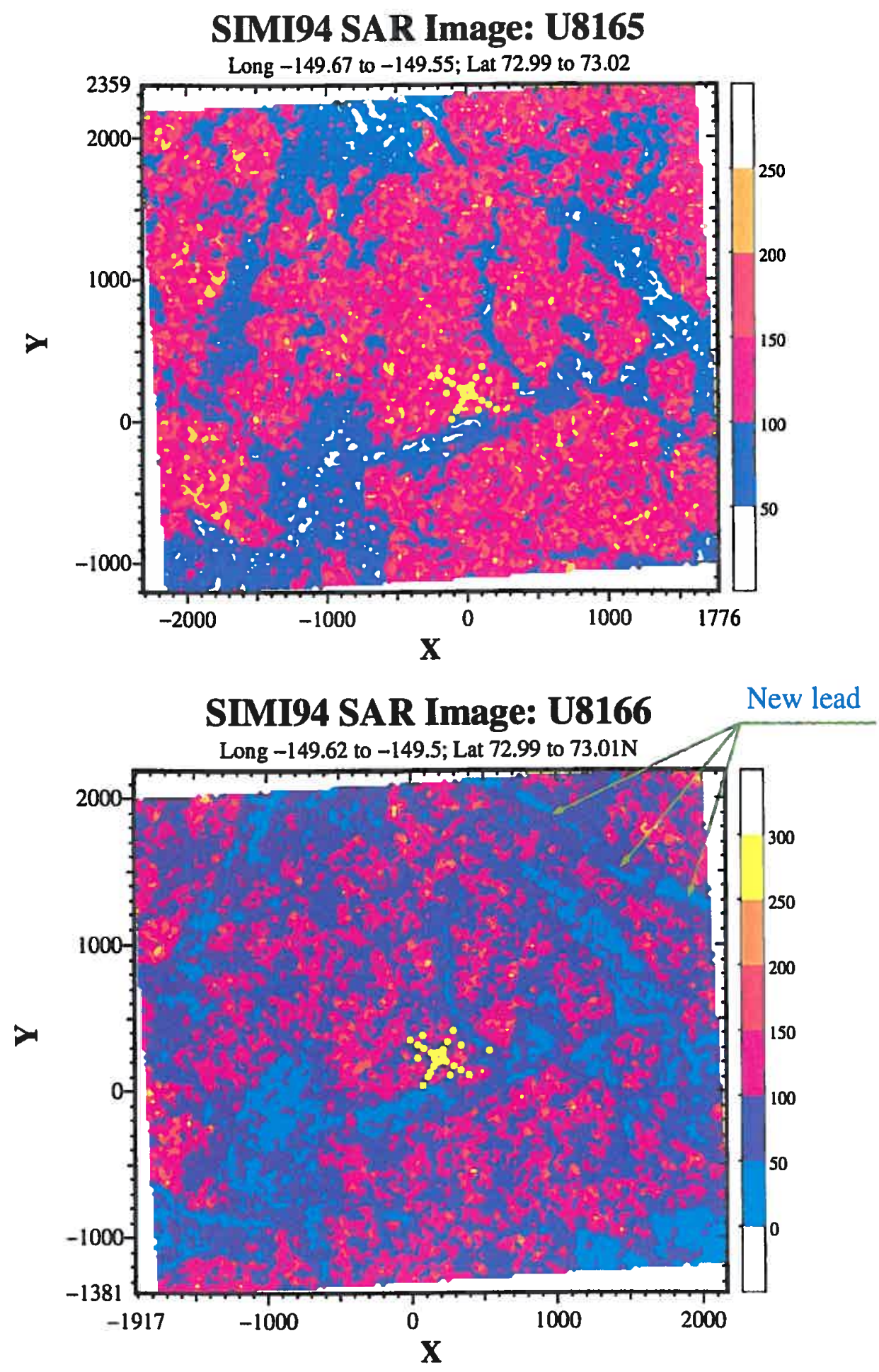

Figure 4-1: Satellite synthetic aperture radar images of SIMI-94 site. The upper plot was recorded at 7.11 am GMT on April 17, 1994. The lower plot was recorded at $9.48 \mathrm{pm}$ GMT on April 18, 1994. The yellow dots near the center of each image show the location of the main hydrophone array (see Fig. 2-2). The new lead is evident as a darker NW-SE strip to the NE of the array on the lower plot, while there is nothing remarkable in the corresponding part of the upper plot. 


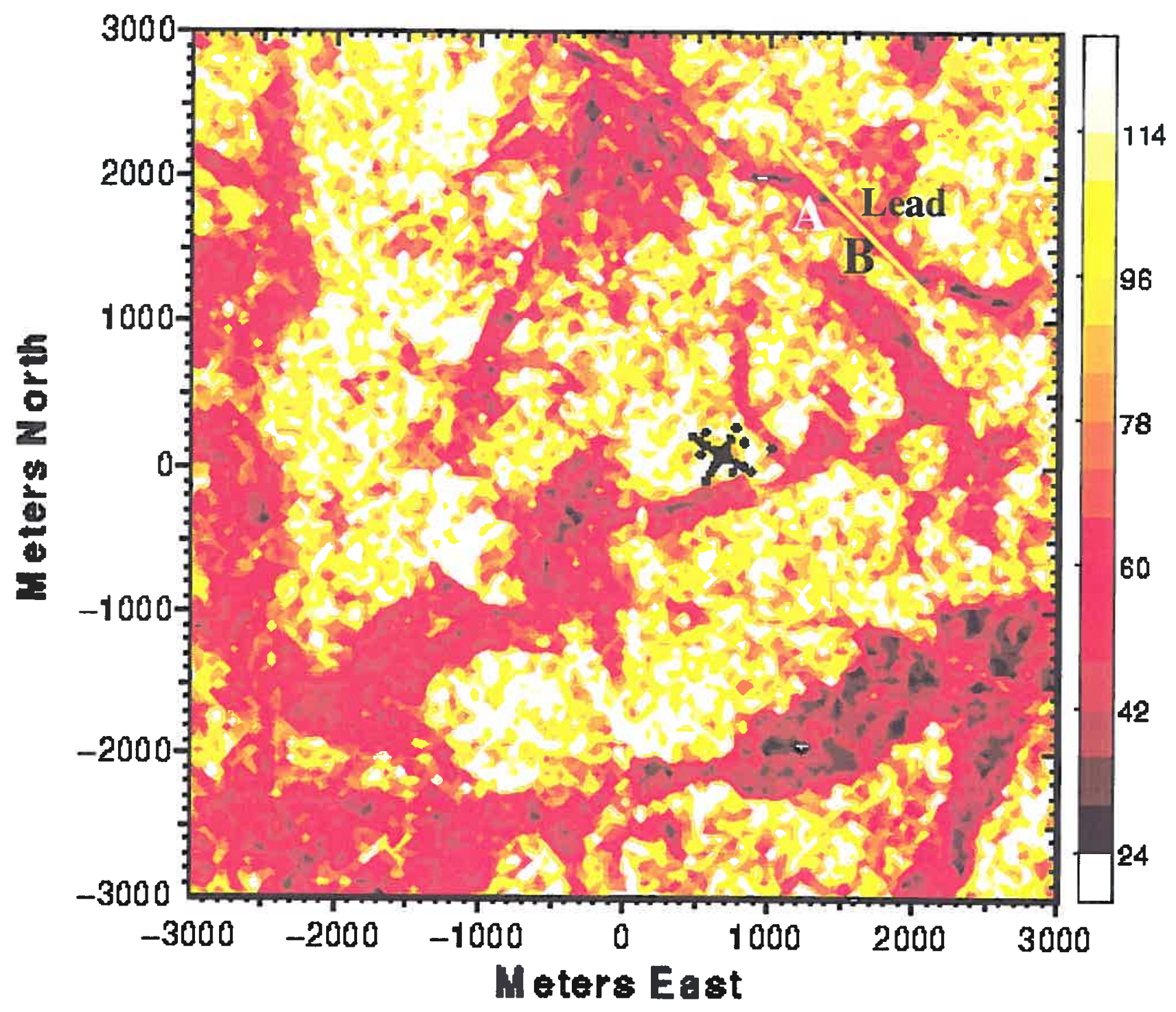

Figure 4-2: High-resolution satellite synthetic aperture radar image of SIMI-94 site recorded at $9.48 \mathrm{pm}$ GMT on April 18, 1994. The black dots near the center of the image show the location of the main hydrophone array (see Fig. 2-2). The new lead is evident as a darker NW-SE strip to the NE of the array, with the local direction indicated by the solid line. Letters $\mathbf{A}$ and $\mathbf{B}$ indicate the approximate locations of the geophone arrays. 


\begin{tabular}{||c|c|c|c||}
\hline $\begin{array}{c}\text { Event } \\
\text { Number }\end{array}$ & $\begin{array}{c}\text { Starting } \\
\text { time, sec. }\end{array}$ & $\begin{array}{c}\text { Ending } \\
\text { time, sec. }\end{array}$ & $\begin{array}{c}\text { Duration } \\
\text { sec. }\end{array}$ \\
\hline \hline 1 & 41.0 & 45.2 & 4.2 \\
2 & 52.5 & 56.7 & 4.2 \\
3 & 62.0 & 66.2 & 4.2 \\
4 & 72.5 & 76.7 & 4.2 \\
5 & 82.0 & 86.2 & 4.2 \\
6 & 93.0 & 97.2 & 4.2 \\
7 & 103.0 & 107.0 & 4.2 \\
8 & 113.0 & 117.0 & 4.2 \\
\hline
\end{tabular}

Table 4.1: Ice events timing intervals

\subsection{The experimental setup and the geophone data}

The data in which evidence for edge waves was found was collected in the vicinity of a major lead formed April 17-18, 1994. This can be seen from two SAR ${ }^{1}$ satellite images from the Alaskan SAR Facility taken on those two dates (see the Fig. 4-1). This lead can be observed in the upper right corner of the lower plot of this figure (taken on April 18), while there is nothing remarkable in the corresponding part of the upper plot. The closer look of this lead is given on Fig. 4-2 (also recorded on April 18). On this figure the predominantly SE-NW direction of the lead is indicated by a solid line, and the surveillance array position is superimposed as well. At its closest point, the distance from the camp to the lead is approximately $2 \mathrm{~km}$. Two RLAM geophone arrays, each having 5 geophones and 1 hydrophone, were deployed in the immediate vicinity of the lead on its SW edge, as indicated in Fig. 4-3. Each geophone measured three components of velocity of ice surface movement, and the hydrophone measured the acoustic pressure in the water. The aperture (radius) of the two geophone clusters was approximately $50 \mathrm{~m}$, and the separation along the lead was $400 \mathrm{~m}$.

Fig. 4-4 shows data from 2 geophones in the site $\mathrm{A}$ cluster. The quasiperiodicity of the signal is evident both on the time series plot and on the autocorre-

\footnotetext{
${ }^{1}$ SAR stands for Synthetic Aperture Radar
} 


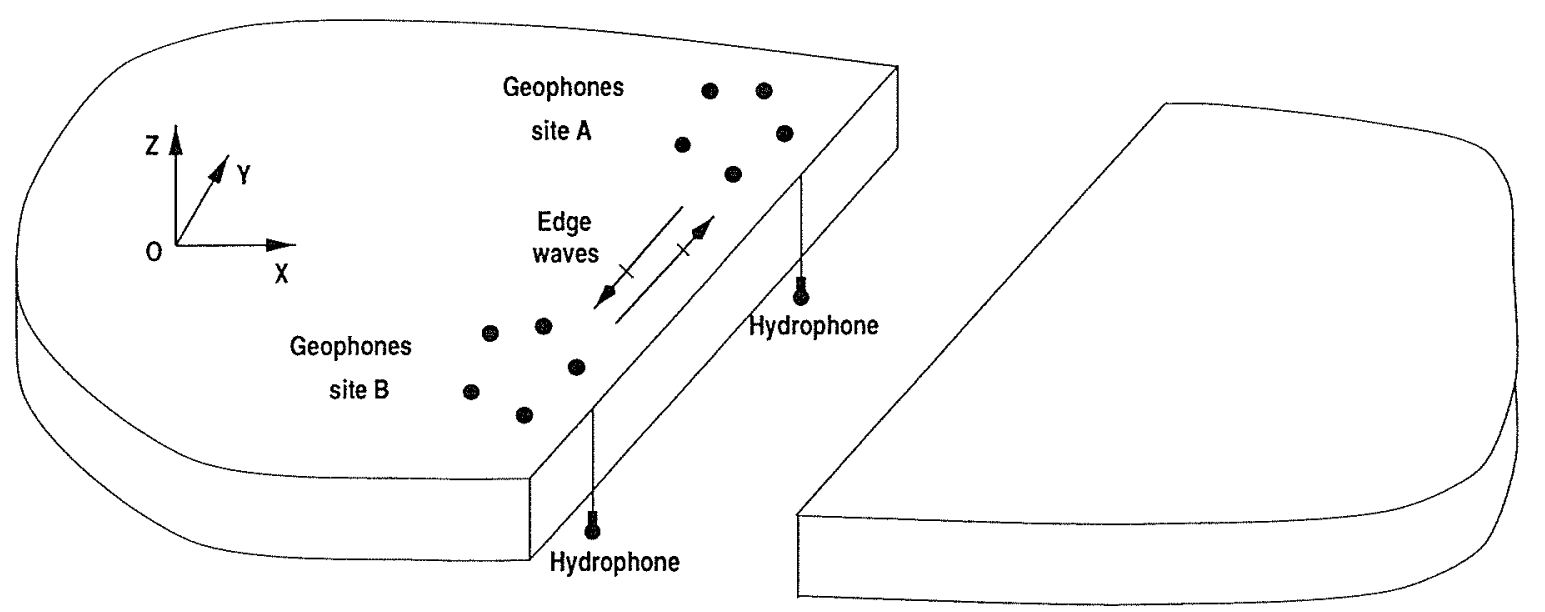

Figure 4-3: Geophone arrays A and B deployed on SW shore of new lead $2 \mathrm{~km} \mathrm{NE}$ of camp. Hydrophones were deployed in the lead at a depth of $60 \mathrm{~m}$. The approximate directions of the 3 geophone axes are indicated by the coordinate system $(X, Y, Z)$. The aperture (radius) of both geophone clusters was approximately $50 \mathrm{~m}$, and the separation between them along the lead was $400 \mathrm{~m}$.

lation plot for the Y-component of the ice particle velocity recorded by the geophone \#1b from site A (see Fig. 4-5). The frequency-wavenumber processing described below was performed event by event, with the event intervals specified in Table 4.1.

\subsection{Data processing scheme}

The wavenumber processing is based on conventional plane wave beamforming using estimated covariance matrices. The sample covariance matrix $\hat{\mathbf{S}}_{\mathbf{x}}(\omega)$ at angular frequency $\omega$ is obtained from the set of complex Fourier component of the signal, $X_{i}^{l}(\omega)$, as

$$
\left(\hat{\mathrm{S}}_{\mathbf{x}}(\omega)\right)_{i j}=\frac{1}{M} \sum_{l=1}^{M}\left[X_{i}^{l}(\omega) \cdot X_{j}^{l *}(\omega)\right]
$$

where $i, j$ represent the sensor numbers, $l$ is the segment number $(l \leq M)$ and the asterisk denotes complex conjugation. To obtain each frequency component, each segment of the raw data is tapered by a Hamming window and Fourier transformed using an FFT algorithm. To avoid energy leakage at the edges of the taper window, the segments are chosen to overlap by $50 \%$. Now the estimate of the power spectrum 


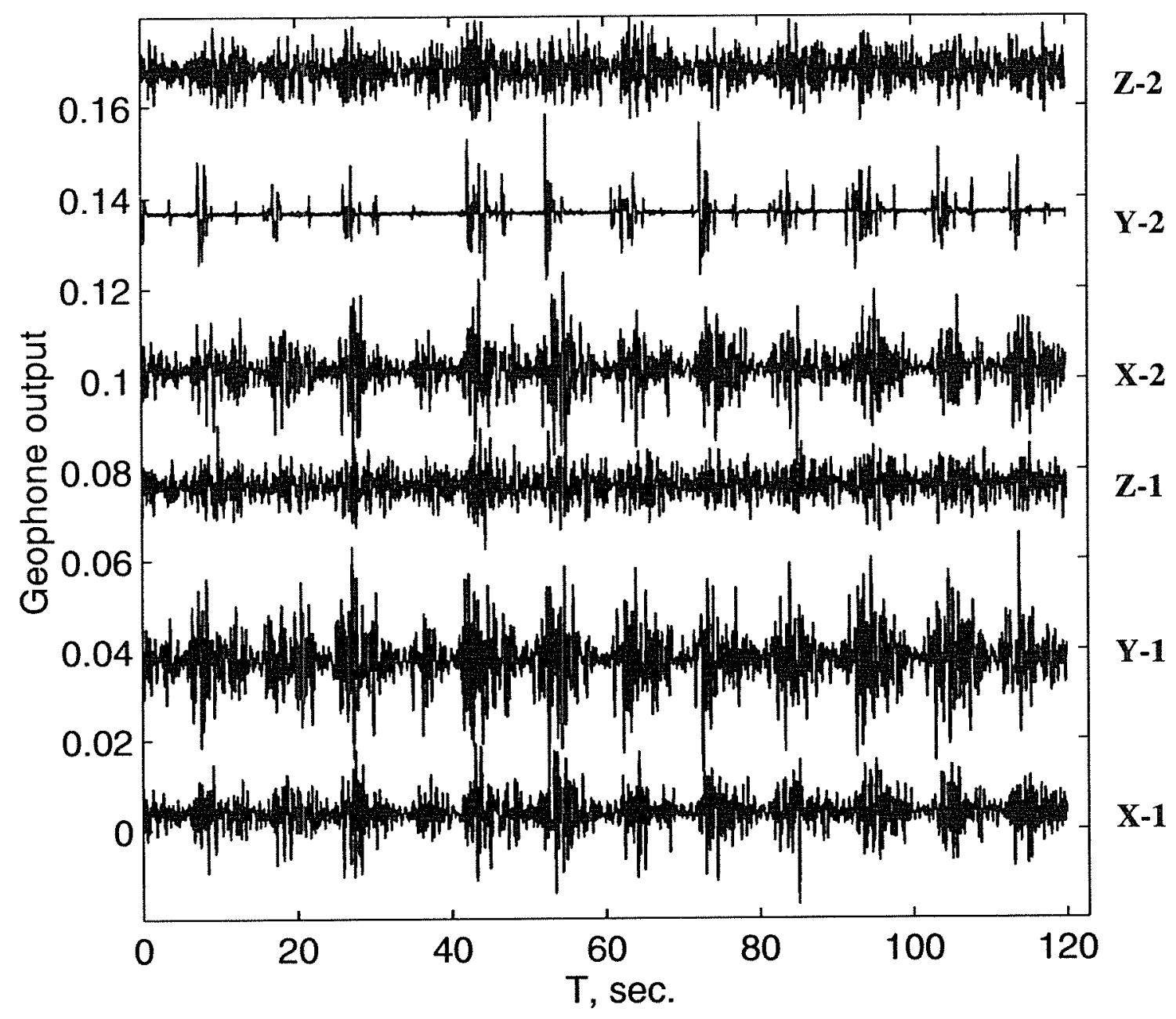

Figure 4-4: Outputs of 2 geophones from site $\mathbf{A}$. The labeling $(\mathrm{X}, \mathrm{Y}, \mathrm{Z})$ represents the geophone components consistent with Fig. 4-3 (X-axis is towards the lead, Y-axis parallel to the lead). Event intervals are specified in Table 4.1. 


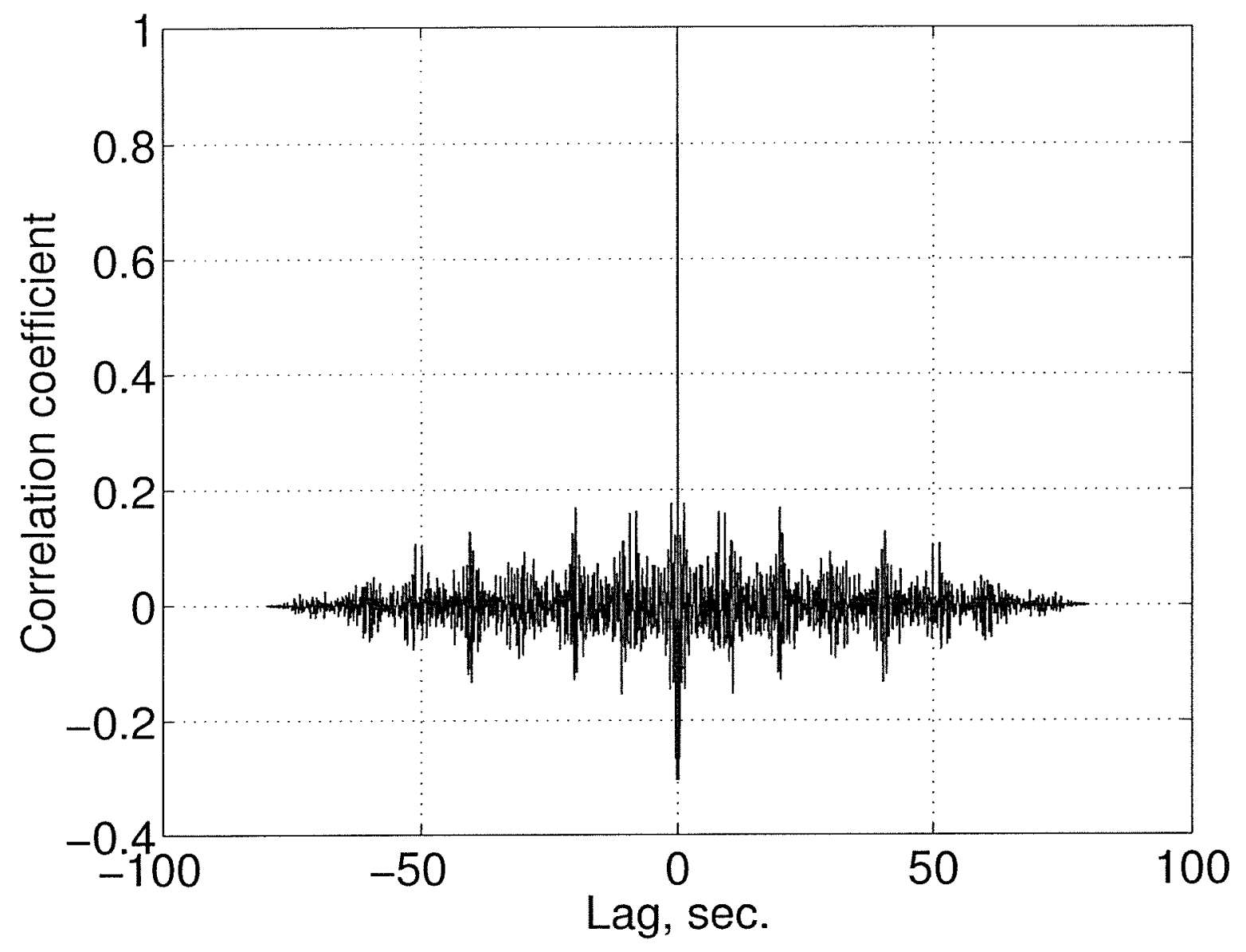

Figure 4-5: Autocorrelation for the $\mathrm{Y}$-component of the ice particle velocity recorded by the geophone \#1b from site $\mathrm{A}$. The time interval (from $40 \mathrm{sec}$. to $120 \mathrm{sec}$.) covers all 8 events from table 4.1 . 


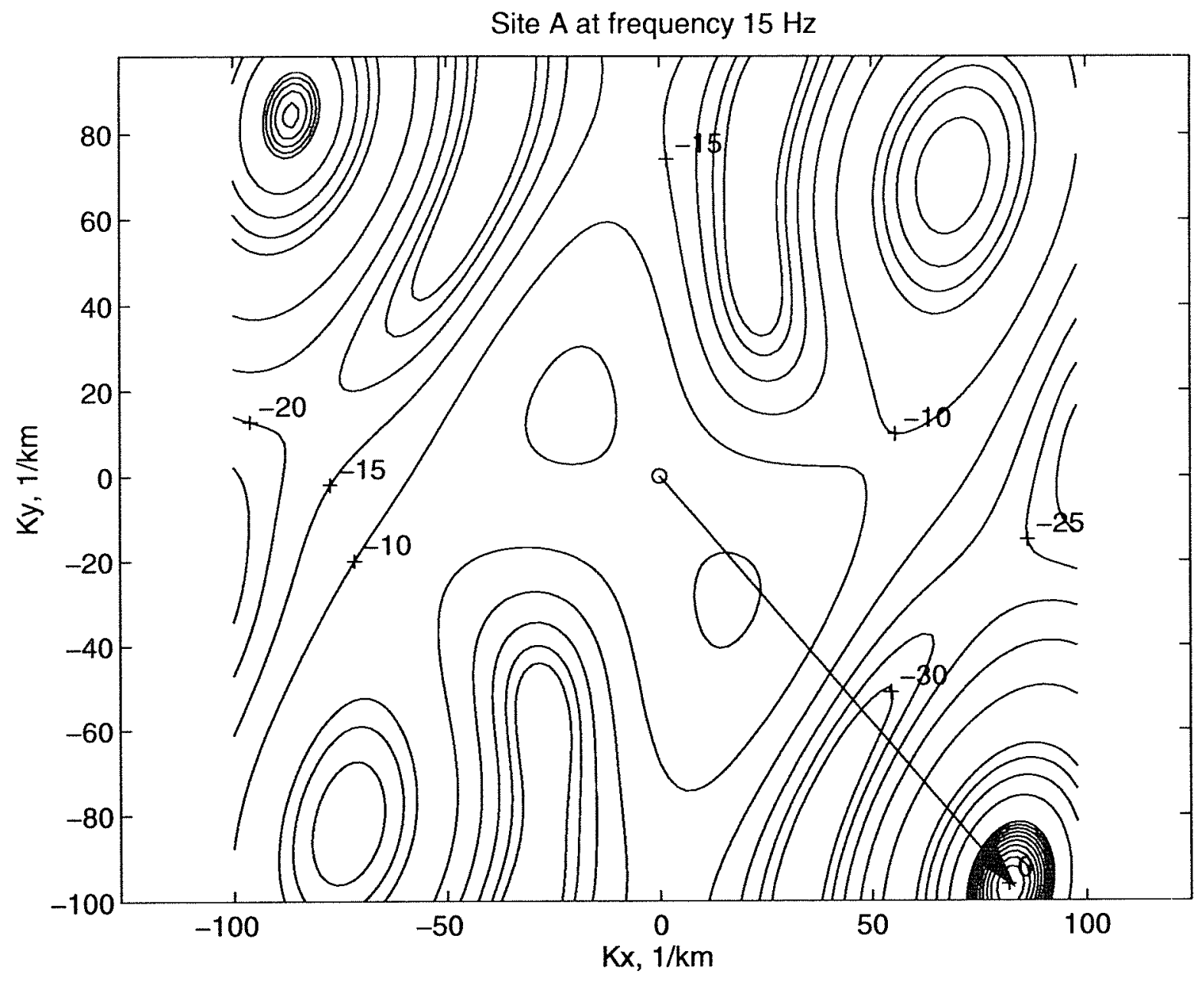

Figure 4-6: Two-dimensional, horizontal wavenumber spectrum for event \#2 (site A, $\mathrm{Y}$-axis outputs of 5 geophones) at $15 \mathrm{~Hz}$. 
at frequency $\omega$ is

$$
P_{\text {conv }}(\omega, \vec{k})=\left(E^{+} \cdot \hat{\mathrm{S}}_{\mathbf{x}} \cdot E\right) / N^{2}
$$

with $E$ being the array steering vector

$$
E=E(\vec{k})=\left(\begin{array}{c}
e^{-j \vec{k}^{T} \vec{x}_{1}} \\
\vdots \\
e^{-j \vec{k}^{T} \vec{x}_{N}}
\end{array}\right)
$$

Here $\vec{k}$ is the horizontal wavenumber vector in the 'look' direction, $\vec{x}_{i}$ is the location of the $i$-th sensor, and $N$ is the total number of sensors. Each component of the geophone output is processed separately, which implies $N=5$ (number of geophones in each array). For each frequency the estimated 2-D wavenumber spectrum is contoured, and the maxima corresponding to the various arrivals are identified. Fig. 4-6 shows a characteristic example at one of the frequencies $(15 \mathrm{~Hz})$ used in the processing, showing two predominant propagation directions, towards the SE and towards the NW, consistent with the lead orientation.

Particularly strong evidence for the 'edge wave' phenomenon was found in events \#2 (Fig. 4-6) and \#3 at site A. The frequency dependence of the propagation direction for the two events are shown in Fig. 4-7. The directions are obviously identical to the propagation directions back and forth along the lead, indicated by the solid lines. The following numbers confirm that conclusion. For the event \#3 (crosses) the mean direction is $m_{3}=126.5^{\circ}$ with $\sigma_{3}=5.5^{\circ}$, while for the event \#2 - the mean $m_{2}=-47.5^{\circ}$ and $\sigma_{2}=2.5^{\circ}$. We see that

$$
\left|180^{\circ}-\left(m_{3}-m_{2}\right)\right|<\left(\sigma_{3}+\sigma_{2}\right)
$$

\footnotetext{
${ }^{2} \sigma$ is the square root of standard deviation about the mean value
} 


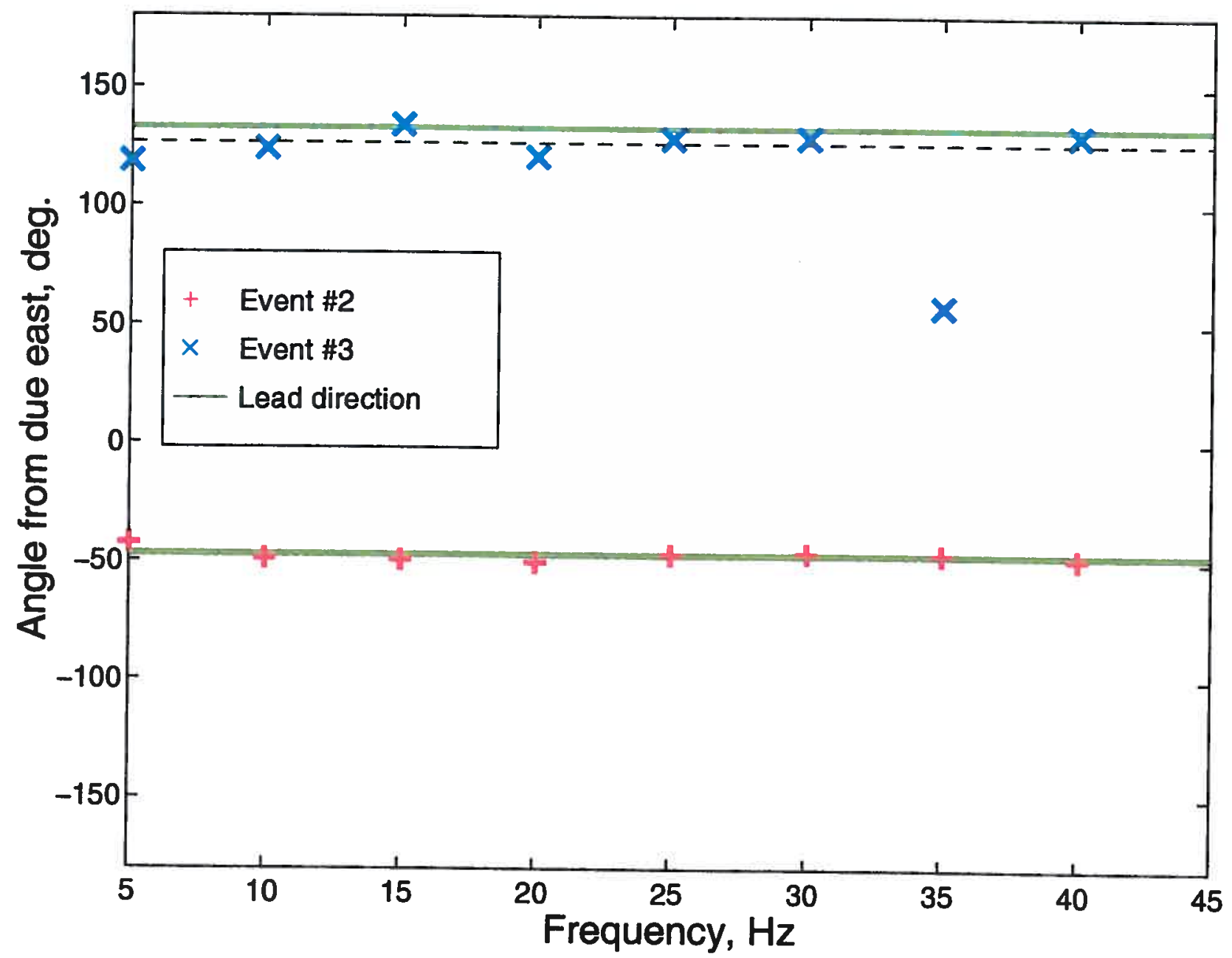

Figure 4-7: Directions of wave arrivals for ice events \#2 and \#3. The $y$-axis indicates the angle $\alpha$ of propagation, counter-clockwise from due East. Dashed lines mark the mean angle of propagation, while the solid lines mark the prevailing local lead direction as indicated in Fig. 4-2. 


\subsection{Theoretical model}

\subsubsection{General equations of motion in elastic medium}

The properties of the operator $\nabla$

We will need most of the properties of the operator $\nabla$ for the following derivation:

$$
\begin{aligned}
& \nabla \cdot(\nabla \phi)=\nabla^{2} \phi \\
& \nabla(\nabla \cdot \Psi)=\nabla^{2} \Psi+\nabla \times(\nabla \times \Psi) \\
& \nabla \times(\nabla \phi)=0 \\
& \nabla \cdot(\nabla \times \Psi)=0
\end{aligned}
$$

The original equation of motion in homogeneous elastic medium

The original equation of motion in homogeneous elastic medium is

$$
(\lambda+\mu) \nabla(\nabla \cdot \mathbf{u})+\mu \nabla^{2} \mathbf{u}-\rho \ddot{\mathbf{u}}=0
$$

where

$$
\begin{aligned}
& \mathbf{u}=\text { displacement vector, } \\
& \rho \quad=\text { the density of the medium, } \\
& \lambda, \mu=\text { Lamé coefficients of the medium. }
\end{aligned}
$$

Using Equation (4.3) we get

$$
\nabla^{2} \mathbf{u}=\nabla(\nabla \cdot \mathbf{u})-\nabla \times \nabla \times \mathbf{u}
$$

and the new form of the equation of motion is

$$
(\lambda+2 \mu) \nabla(\nabla \cdot \mathbf{u})-\mu \nabla \times \nabla \times \mathbf{u}-\rho \ddot{\mathbf{u}}=0
$$




\section{The potentials}

The displacement field (as any vector field) can be described by a scalar potential and a vector potential:

$$
\mathbf{u}=\nabla \phi+\nabla \times \Psi
$$

In Equation (4.8) the vector potential $\Psi$ should satisfy the gauge condition:

$$
\nabla \cdot \Psi=0
$$

This gauge condition guarantees that the shear wave components are equivoluminal waves.

As a result we can rewrite the equation of motion in the following form:

$$
\begin{gathered}
(\lambda+2 \mu) \nabla(\nabla \cdot(\nabla \phi)+\nabla \cdot(\nabla \times \Psi))-\mu \nabla \times \nabla \times(\nabla \phi)- \\
-\mu \nabla \times \nabla \times(\nabla \times \Psi)-\rho(\nabla \ddot{\phi}+\nabla \times \ddot{\Psi})
\end{gathered}
$$

Now using Equations (4.3-4.5) ${ }^{3}$ we obtain from Equation (4.10)

$$
(\lambda+2 \mu) \nabla\left(\nabla^{2} \phi\right)-\mu \nabla \times\left(\nabla(\nabla \cdot \Psi)-\nabla^{2} \Psi\right)-\rho(\nabla \ddot{\phi}+\nabla \times \ddot{\Psi})=0
$$

Using gauge condition Equation (4.9) we have

$$
(\lambda+2 \mu) \nabla\left(\nabla^{2} \phi\right)-\rho \nabla \ddot{\phi}+\mu \nabla \times\left(\nabla^{2} \Psi\right)-\rho \nabla \times \ddot{\Psi}=0
$$

Using standard expressions for propagation speeds in homogeneous medium of com-

\footnotetext{
${ }^{3}$ compare fourth term in Equation (4.10) and second term in Equation (4.11)
} 
pressional $\left(c_{p}\right)$ and shear $\left(c_{s}\right)$ waves

$$
\begin{aligned}
& c_{p}=\sqrt{\frac{\lambda+2 \mu}{\rho}} \\
& c_{s}=\sqrt{\frac{\mu}{\rho}}
\end{aligned}
$$

we get the following form of equation of motion

$$
c_{p}^{2} \rho \nabla\left(\nabla^{2} \phi-\frac{1}{c_{p}^{2}} \ddot{\phi}\right)+c_{s}^{2} \rho \nabla \times\left(\nabla^{2} \Psi-\frac{1}{c_{s}^{2}} \ddot{\Psi}\right)=0 .
$$

The solution to this equation will be given by the potentials $\phi$ and $\Psi$ that satisfy the following equations:

$$
\begin{aligned}
\nabla^{2} \phi-\frac{1}{c_{p}^{2}} \ddot{\phi} & =0 \\
\nabla^{2} \Psi-\frac{1}{c_{s}^{2}} \ddot{\Psi} & =0 .
\end{aligned}
$$

For harmonic field with frequency $\omega$ we have

$$
\begin{aligned}
& \ddot{\phi}=-\omega^{2} \phi \\
& \ddot{\Psi}=-\omega^{2} \Psi,
\end{aligned}
$$

and Equation s(4.14) and (4.15) take the form of Helmholtz equation:

$$
\begin{aligned}
\nabla^{2} \phi+\frac{\omega^{2}}{c_{p}^{2}} \ddot{\phi} & =0 \\
\nabla^{2} \Psi+\frac{\omega^{2}}{c_{s}^{2}} \ddot{\Psi} & =0
\end{aligned}
$$

The vector displacement potential $\Psi$ can be represented as follows:

$$
\Psi=\nabla \times(0,0, \Lambda)+\left(0,0, \Psi_{z}\right) .
$$

Introducing compressional media wavenumber $\left(k_{p}\right)$ and shear media wavenumber $\left(k_{s}\right)$ 


$$
\begin{aligned}
& k_{p}=\frac{\omega}{c_{p}} \\
& k_{s}=\frac{\omega}{c_{s}},
\end{aligned}
$$

we obtain the following form of the Helmholtz equations for the displacement potentials of the homogeneous part of the field

$$
\begin{aligned}
& \left(\nabla^{2}+k_{p}^{2}\right) \phi(x, y, z)=0 \\
& \left(\nabla^{2}+k_{s}^{2}\right) \Lambda(x, y, z)=0 \\
& \left(\nabla^{2}+k_{s}^{2}\right) \Psi_{z}(x, y, z)=0 .
\end{aligned}
$$

Let us apply the Fourier Transform with respect to $y$

$$
\begin{aligned}
& F\left(x, k_{y}, z\right)=\int_{-\infty}^{+\infty} f(x, y, z) e^{i k_{y} y} \mathrm{~d} y \\
& f(x, y, z)=\frac{1}{2 \pi} \int_{-\infty}^{+\infty} F\left(x, k_{y}, z\right) e^{-i k_{y} y} \mathrm{~d} k_{y}
\end{aligned}
$$

on the Helmholtz equations to get:

$$
\begin{aligned}
& \left(\frac{\partial^{2}}{\partial x^{2}}+\frac{\partial^{2}}{\partial z^{2}}-\left(k_{y}^{2}-k_{p}^{2}\right)\right) \phi\left(x, k_{y}, z\right)=0 \\
& \left(\frac{\partial^{2}}{\partial x^{2}}+\frac{\partial^{2}}{\partial z^{2}}-\left(k_{y}^{2}-k_{p}^{2}\right)\right) \Lambda\left(x, k_{y}, z\right)=0 \\
& \left(\frac{\partial^{2}}{\partial x^{2}}+\frac{\partial^{2}}{\partial z^{2}}-\left(k_{y}^{2}-k_{p}^{2}\right)\right) \Psi_{z}\left(x, k_{y}, z\right)=0
\end{aligned}
$$

Similarly, after applying the Fourier Transform with respect to $x$, we obtain:

$$
\begin{aligned}
& \left(\frac{\partial^{2}}{\partial z^{2}}-\alpha^{2}\right) \phi\left(k_{x}, k_{y}, z\right)=0 \\
& \left(\frac{\partial^{2}}{\partial z^{2}}-\beta^{2}\right) \Lambda\left(k_{x}, k_{y}, z\right)=0 \\
& \left(\frac{\partial^{2}}{\partial z^{2}}-\beta^{2}\right) \Psi_{z}\left(k_{x}, k_{y}, z\right)=0
\end{aligned}
$$


where

$$
\begin{aligned}
& \alpha=\sqrt{k_{x}^{2}+k_{y}^{2}-k_{p}^{2}} \\
& \beta=\sqrt{k_{x}^{2}+k_{y}^{2}-k_{s}^{2}} .
\end{aligned}
$$

Solving above equations for the displacement potentials we get:

$$
\begin{aligned}
& \phi\left(k_{x}, k_{y}, z\right)=A^{-}\left(k_{x}, k_{y}\right) e^{-\alpha z}+A^{+}\left(k_{x}, k_{y}\right) e^{\alpha z} \\
& \Psi_{z}\left(k_{x}, k_{y}, z\right)=B^{-}\left(k_{x}, k_{y}\right) e^{-\beta z}+B^{+}\left(k_{x}, k_{y}\right) e^{\beta z} \\
& \Lambda\left(k_{x}, k_{y}, z\right)=C^{-}\left(k_{x}, k_{y}\right) e^{-\beta z}+C^{+}\left(k_{x}, k_{y}\right) e^{\beta z}
\end{aligned}
$$

\section{Expressions for displacement components}

Now for displacement we have the following expression

$$
\begin{aligned}
\mathbf{u}(x, y, z) & =\nabla \phi(x, y, z)+\nabla \times\left(\nabla \times(0,0, \Lambda)+\left(0,0, \Psi_{z}\right)\right) \\
& =\nabla \phi+\nabla \times\left(\frac{\partial}{\partial y} \Lambda,-\frac{\partial}{\partial x} \Lambda, \Psi_{z}\right)
\end{aligned}
$$

Introducing the notation $u \triangleq u_{x}, \quad v \triangleq u_{y}, \quad w \triangleq u_{z}$ and

$$
\begin{aligned}
& S_{A}^{ \pm}=A^{-}\left(k_{x}, k_{y}\right) e^{-\alpha z} \pm A^{+}\left(k_{x}, k_{y}\right) e^{\alpha z} \\
& S_{B}^{ \pm}=B^{-}\left(k_{x}, k_{y}\right) e^{-\beta z} \pm B^{+}\left(k_{x}, k_{y}\right) e^{\beta z} \\
& S_{C}^{ \pm}=C^{-}\left(k_{x}, k_{y}\right) e^{-\beta z} \pm C^{+}\left(k_{x}, k_{y}\right) e^{\beta z}
\end{aligned}
$$

we get

$$
\begin{aligned}
u & =\frac{\partial \phi}{\partial x}+\frac{\partial \Psi_{z}}{\partial y}+\frac{\partial^{2} \Lambda}{\partial x \partial z}=-i k_{x} S_{A}^{+}-i k_{y} S_{B}^{+}+i k_{x} \beta S_{C}^{-} \\
v & =\frac{\partial \phi}{\partial y}-\frac{\partial \Psi_{z}}{\partial x}+\frac{\partial^{2} \Lambda}{\partial y \partial z}=-i k_{y} S_{A}^{+}+i k_{x} S_{B}^{+}+i k_{y} \beta S_{C}^{-} \\
w & =\frac{\partial \phi}{\partial z}-\left(\frac{\partial^{2}}{\partial x^{2}}+\frac{\partial^{2}}{\partial y^{2}}\right) \Lambda=-\alpha S_{A}^{-}+\left(k_{x}^{2}+k_{y}^{2}\right) S_{C}^{+}
\end{aligned}
$$




\section{Expressions for stresses}

Using

$$
\epsilon_{j k}=\frac{1}{2}\left(\frac{\partial u_{j}}{\partial x_{k}}+\frac{\partial u_{k}}{\partial x_{j}}\right)
$$

and

$$
\sigma_{i j}=\lambda \frac{\partial u_{k}}{\partial x_{k}} \delta_{i j}+\mu\left(\frac{\partial u_{i}}{\partial x_{j}}+\frac{\partial u_{j}}{\partial x_{i}}\right)
$$

we have for stresses:

$$
\begin{aligned}
\sigma_{z z} & =(\lambda+2 \mu) \frac{\partial w}{\partial z}+\lambda\left(\frac{\partial u}{\partial x}+\frac{\partial v}{\partial y}\right) \\
\sigma_{z x} & =\mu\left(\frac{\partial w}{\partial x}+\frac{\partial u}{\partial z}\right) \\
\sigma_{z y} & =\mu\left(\frac{\partial w}{\partial y}+\frac{\partial v}{\partial z}\right) \\
\sigma_{x x} & =(\lambda+2 \mu) \frac{\partial u}{\partial x}+\lambda\left(\frac{\partial v}{\partial y}+\frac{\partial w}{\partial z}\right) \\
\sigma_{x y} & =\mu\left(\frac{\partial u}{\partial y}+\frac{\partial v}{\partial x}\right) \\
\sigma_{x z} & =\mu\left(\frac{\partial u}{\partial z}+\frac{\partial w}{\partial x}\right)
\end{aligned}
$$

Note, that $\sigma_{z x}$, of course, is always equal to $\sigma_{x z}$. Both of them are kept in the derivation for convenience of formulating the boundary condition at the vertical free face of the plate at $x=0$.

Substituting Equations (4.36-4.38) into above expressions and introducing the following notation for horizontal wavenumber

$$
\gamma=\sqrt{k_{x}^{2}+k_{y}^{2}}
$$


we obtain:

$$
\begin{aligned}
& \sigma_{z z}=(\lambda+2 \mu)\left(\alpha^{2} S_{A}^{+}-\gamma^{2} \beta S_{C}^{-}\right)+\lambda\left(-k_{x}^{2} S_{A}^{+}-k_{x} k_{y} S_{B}^{+}+k_{x}^{2} \beta S_{C}^{-}\right)+ \\
& +\lambda\left(-k_{y}^{2} S_{A}^{+}+k_{x} k_{y} S_{B}^{+}+k_{y}^{2} \beta S_{C}^{-}\right) \\
& =\mu\left(2 \gamma^{2}-k_{s}^{2}\right) S_{A}^{+}-2 \mu \gamma^{2} \beta S_{C}^{-} \text {, } \\
& \sigma_{z x}=\mu\left(i k_{x} \alpha S_{A}^{-}-i k_{x} \gamma^{2} S_{C}^{+}\right)+\mu\left(i k_{x} \alpha S_{A}^{-}+i k_{y} \beta S_{B}^{-}-i k_{x} \beta^{2} S_{C}^{+}\right) \\
& =2 i \mu k_{x} \alpha S_{A}^{-}+i \mu k_{y} \beta S_{B}^{-}-i \mu k_{x}\left(2 \gamma^{2}-k_{s}^{2}\right) S_{C}^{+}, \\
& \sigma_{z y}=\mu\left(i k_{y} \alpha S_{A}^{-}-i k_{y} \gamma^{2} S_{C}^{+}\right)+\mu\left(i k_{y} \alpha S_{A}^{-}-i k_{y} \beta^{2} S_{C}^{+}-i k_{x} \beta S_{B}^{-}\right) \\
& =2 i \mu k_{y} \alpha S_{A}^{-}-i \mu k_{x} \beta S_{B}^{-}-i \mu k_{y}\left(2 \gamma^{2}-k_{s}^{2}\right) S_{C}^{+}, \\
& \sigma_{x x}=(\lambda+2 \mu)\left(-k_{x}^{2} S_{A}^{+}-k_{x} k_{y} S_{B}^{+}+k_{x}^{2} \beta S_{C}^{-}\right)+ \\
& +\lambda\left(-k_{y}^{2} S_{A}^{+}+k_{x} k_{y} S_{B}^{+}+k_{y}^{2} \beta S_{C}^{-}\right)+\lambda\left(\alpha^{2} S_{A}^{+}-\gamma^{2} \beta S_{C}^{-}\right) \\
& =\left[-k_{x}^{2}(\lambda+2 \mu)-\lambda k_{y}^{2}+\lambda \alpha^{2}\right] S_{A}^{+}+\left[-k_{x} k_{y}(\lambda+2 \mu)+\lambda k_{x} k_{y}\right] S_{B}^{+}+ \\
& +\left[k_{x}^{2} \beta(\lambda+2 \mu)+\lambda k_{y}^{2} \beta-\lambda \gamma^{2} \beta\right] S_{C}^{-} \\
& =-\left(2 \mu k_{x}^{2}+\lambda k_{p}^{2}\right) S_{A}^{+}-2 \mu k_{x} k_{y} S_{B}^{+}+2 \mu k_{x}^{2} \beta S_{C}^{-}, \\
& \sigma_{x y}=\mu\left(-k_{x} k_{y} S_{A}^{+}-k_{y}^{2} S_{B}^{+}+k_{x} k_{y} \beta S_{C}^{-}\right)+\mu\left(-k_{x} k_{y} S_{A}^{+}+k_{x}^{2} S_{B}^{+}+k_{x} k_{y} \beta S_{C}^{-}\right) \\
& =-2 \mu k_{x} k_{y} S_{A}^{+}+\mu\left(k_{x}^{2}-k_{y}^{2}\right) S_{B}^{+}+2 \mu k_{x} k_{y} \beta S_{C}^{-}, \\
& \sigma_{x z}=i \mu k_{x} \alpha S_{A}^{-}+i \mu k_{y} \beta S_{B}^{-}-i \mu k_{x} \beta^{2} S_{C}^{+}+\mu\left(i k_{x} \alpha S_{A}^{-}-i k_{x} \gamma^{2} S_{C}^{+}\right) \\
& =2 i \mu k_{x} \alpha S_{A}^{-}+i \mu k_{y} \beta S_{B}^{-}-i \mu k_{x}\left(2 \gamma^{2}-k_{s}^{2}\right) S_{C}^{+} .
\end{aligned}
$$


Now we have the final expressions for stresses:

$$
\begin{aligned}
& \sigma_{z z}=\mu\left(2 \gamma^{2}-k_{s}^{2}\right) S_{A}^{+}-2 \mu \gamma^{2} \beta S_{C}^{-} \\
& \sigma_{z x}=2 i \mu k_{x} \alpha S_{A}^{-}+i \mu k_{y} \beta S_{B}^{-}-i \mu k_{x}\left(2 \gamma^{2}-k_{s}^{2}\right) S_{C}^{+} \\
& \sigma_{z y}=2 i \mu k_{y} \alpha S_{A}^{-}-i \mu k_{x} \beta S_{B}^{-}-i \mu k_{y}\left(2 \gamma^{2}-k_{s}^{2}\right) S_{C}^{+} \\
& \sigma_{x x}=-\left(2 \mu k_{x}^{2}+\lambda k_{p}^{2}\right) S_{A}^{+}-2 \mu k_{x} k_{y} S_{B}^{+}+2 \mu k_{x}^{2} \beta S_{C}^{-} \\
& \sigma_{x y}=-2 \mu k_{x} k_{y} S_{A}^{+}+\mu\left(k_{x}^{2}-k_{y}^{2}\right) S_{B}^{+}+2 \mu k_{x} k_{y} \beta S_{C}^{-} \\
& \sigma_{x z}=2 i \mu k_{x} \alpha S_{A}^{-}+i \mu k_{y} \beta S_{B}^{-}-i \mu k_{x}\left(2 \gamma^{2}-k_{s}^{2}\right) S_{C}^{+}
\end{aligned}
$$

We will need Eqs.4.36-4.38 and Eqs.4.45-4.50 to write expressions for boundary conditions at the free vertical face of the plate.

\subsubsection{The fundamental modes of the infinite plate}

As a basis for the edge wave model, the first symmetric and antisymmetric Lamb modes (P-SV) and first symmetric and antisymmetric Love modes (SH) of the infinite plate are assumed to be dominating. This is a reasonable assumption for frequencies below the thickness-shear frequency for the the ice cover, typically of the order 200-300 $\mathrm{Hz}$. The discussion of these modes follows below.

\section{The first symmetric Lamb mode}

For the first symmetric Lamb mode we have the following symmetry conditions:

$$
\begin{aligned}
& A^{+}=A^{-} \triangleq A \\
& B^{+}=B^{-}=0 \\
& C^{+}=-C^{-} \triangleq C .
\end{aligned}
$$


These give the following depth dependencies

$$
\begin{array}{ll}
S_{A}^{+}=2 A \cosh (\alpha z) & S_{A}^{-}=-2 A \sinh (\alpha \tilde{)}) \\
S_{B}^{+}=0 & S_{B}^{-}=0 \\
S_{C}^{+}=2 C \sinh (\beta z) & S_{C}^{-}=-2 C \cosh (\beta z)
\end{array}
$$

In the above expressions constants $A$ and $C$ could be expressed in terms of one constant $A_{\text {sym }}$ using one of the boundary conditions on either one of the horizontal faces of the plate (either at $z=-h$ or at $z=h$ ):

$$
\begin{aligned}
& A=2 \gamma^{2} \beta \cosh (\beta h) A_{\mathrm{sym}} \\
& C=-\left(2 \gamma^{2}-k_{s}^{2}\right) \cosh (\alpha h) A_{\mathrm{sym}}
\end{aligned}
$$

The above depth dependencies give us following expressions for the stress components which are used for the vertical boundary condition at $x=0$ :

$$
\begin{aligned}
& \sigma_{x x}=-2\left(2 \mu k_{x}^{2}+\lambda k_{p}^{2}\right) A \cosh (\alpha z)-4 \mu k_{x}^{2} \beta C \cosh (\beta z) \\
& \sigma_{x y}=-4 \mu k_{x} k_{y} A \cosh (\alpha z)-4 \mu k_{x} k_{y} \beta C \cosh (\beta z) \\
& \sigma_{x z}=-4 i \mu k_{x} \alpha A \sinh (\alpha z)-2 i \mu k_{x}\left(2 \gamma^{2}-k_{s}^{2}\right) C \sinh (\beta z)
\end{aligned}
$$

The dispersion relation for the first symmetric Lamb mode could be easily obtained from the symmetry conditions stated above and the boundary conditions at either one of the horizontal faces of the place (either at $z=-h$ or at $z=h$ ). The derivation of the dispersion relation will be given only for this mode (for other modes it could be done by analogy). We have two equations at $z=h$ :

$$
\begin{gathered}
\left.\sigma_{z z}\right|_{z=h}=0 \\
\left.\sigma_{z x}\right|_{z=h}=0
\end{gathered}
$$


This gives us the following system of equations:

$$
\begin{array}{r}
2 \mu\left(2 \gamma^{2}-k_{s}^{2}\right) A \cosh (\alpha h)+4 \mu \gamma^{2} \beta C \cosh (\beta h)=0, \\
-4 i \mu k_{x} \alpha A \sinh (\alpha h)-2 i \mu k_{x}\left(2 \gamma^{2}-k_{s}^{2}\right) C \sinh (\beta h)=0 .
\end{array}
$$

After dividing the first one by $2 \mu$ and the second one by $-2 i \mu k_{x}$ we get

$$
\begin{aligned}
\left(2 \gamma^{2}-k_{s}^{2}\right) \cosh (\alpha h) A+2 \gamma^{2} \beta \cosh (\beta h) & C=0 \\
2 \alpha \sinh (\alpha h) A-\left(2 \gamma^{2}-k_{s}^{2}\right) \sinh (\beta h) C & =0
\end{aligned}
$$

This system has nontrivial solutions with respect to $A$ and $C$ only when the determinant of the system is zero:

$$
\operatorname{det}\left\|\begin{array}{ll}
\left(2 \gamma^{2}-k_{s}^{2}\right) \cosh (\alpha h) & 2 \gamma^{2} \beta \cosh (\beta h) \\
2 \alpha \sinh (\alpha h) & -\left(2 \gamma^{2}-k_{s}^{2}\right) \sinh (\beta h)
\end{array}\right\|=0
$$

This equation leads to the following form of the dispersion relation for the symmetric Lamb modes

$$
\frac{\tanh \beta h}{\tanh \alpha h}=\frac{4 \gamma^{2} \alpha \beta}{\left(2 \gamma^{2}-k_{s}^{2}\right)^{2}}
$$

We could solve it numerically with respect to $\gamma$. As a result, we would obtain $\gamma$ as a function of frequency $\omega$ after choosing branch corresponding to the first mode:

$$
\gamma \triangleq \mathcal{D}_{S}(\omega)
$$




\section{The first antisymmetric Lamb mode}

For the first antisymmetric Lamb mode we have the following symmetry conditions:

$$
\begin{aligned}
& A^{+}=-A^{-} \triangleq A \\
& B^{+}=B^{-}=0 \\
& C^{+}=C^{-} \triangleq C .
\end{aligned}
$$

These give the following depth dependencies

$$
\begin{array}{ll}
S_{A}^{+}=2 A \sinh (\alpha z) & S_{A}^{-}=-2 A \cosh (\alpha z) \\
S_{B}^{+}=0 & S_{B}^{-}=0 \\
S_{C}^{+}=2 C \cosh (\beta z) & S_{C}^{-}=-2 C \sinh (\beta z)
\end{array}
$$

Again, in the above expressions constants $A$ and $C$ could be expressed in terms of one constant $A_{\text {asym }}$ using one of the boundary conditions on either one of the horizontal faces of the plate (either at $z=-h$ or at $z=h$ ):

$$
\begin{aligned}
& A=2 \gamma^{2} \beta \sinh (\beta h) A_{\text {asym }} \\
& C=-\left(2 \gamma^{2}-k_{s}^{2}\right) \sinh (\alpha h) A_{\text {asym }} .
\end{aligned}
$$

The above depth dependencies give us following expressions for the stress components which are used for the vertical boundary condition at $x=0$ :

$$
\begin{aligned}
& \sigma_{x x}=-2\left(2 \mu k_{x}^{2}+\lambda k_{p}^{2}\right) A \sinh (\alpha z)-4 \mu k_{x}^{2} \beta C \sinh (\beta z) \\
& \sigma_{x y}=-4 \mu k_{x} k_{y} A \sinh (\alpha z)-4 \mu k_{x} k_{y} \beta C \sinh (\beta z) \\
& \sigma_{x z}=-4 i \mu k_{x} \alpha A \cosh (\alpha z)-2 i \mu k_{x}\left(2 \gamma^{2}-k_{s}^{2}\right) 2 C \cosh (\beta z)
\end{aligned}
$$


The dispersion relation for the antisymmetric Lamb modes could be easily obtained from the symmetry conditions stated above and the boundary conditions at either one of the horizontal faces of the place similarly, as above for the symmetric Lamb modes. It has the following form:

$$
\frac{\tanh \beta h}{\tanh \alpha h}=\left(\frac{4 \gamma^{2} \alpha \beta}{\left(2 \gamma^{2}-k_{s}^{2}\right)^{2}}\right)^{-1}
$$

Solving it numerically with respect to $\gamma$ and choosing the branch corresponding to the first mode we get $\gamma$ as a function of frequency $\omega$ :

$$
\gamma \triangleq \mathcal{D}_{A}(\omega)
$$

The plots of the dispersion relations for the first symmetric and antisymmetric Lamb modes of the infinite plate are shown in non-dimensional form on the Fig. 48 where non-dimensional frequency $\Omega$ is plotted versus non-dimensional horizontal wavenumber $\Gamma$. Non-dimensional frequency $\Omega$ and horizontal wavenumber $\Gamma$ are defined by

$$
\begin{aligned}
& \Omega=\frac{2 h}{\pi} k_{s} \\
& \Gamma=\frac{2 h}{\pi} \gamma
\end{aligned}
$$

\section{The first symmetric Love mode}

For the first symmetric Love mode we have the following symmetry conditions:

$$
\begin{aligned}
& A^{+}=A^{-}=0 \\
& B^{+}=B^{-} \triangleq B \\
& C^{+}=C^{-}=0 .
\end{aligned}
$$




\section{First two fundamental Lamb modes}

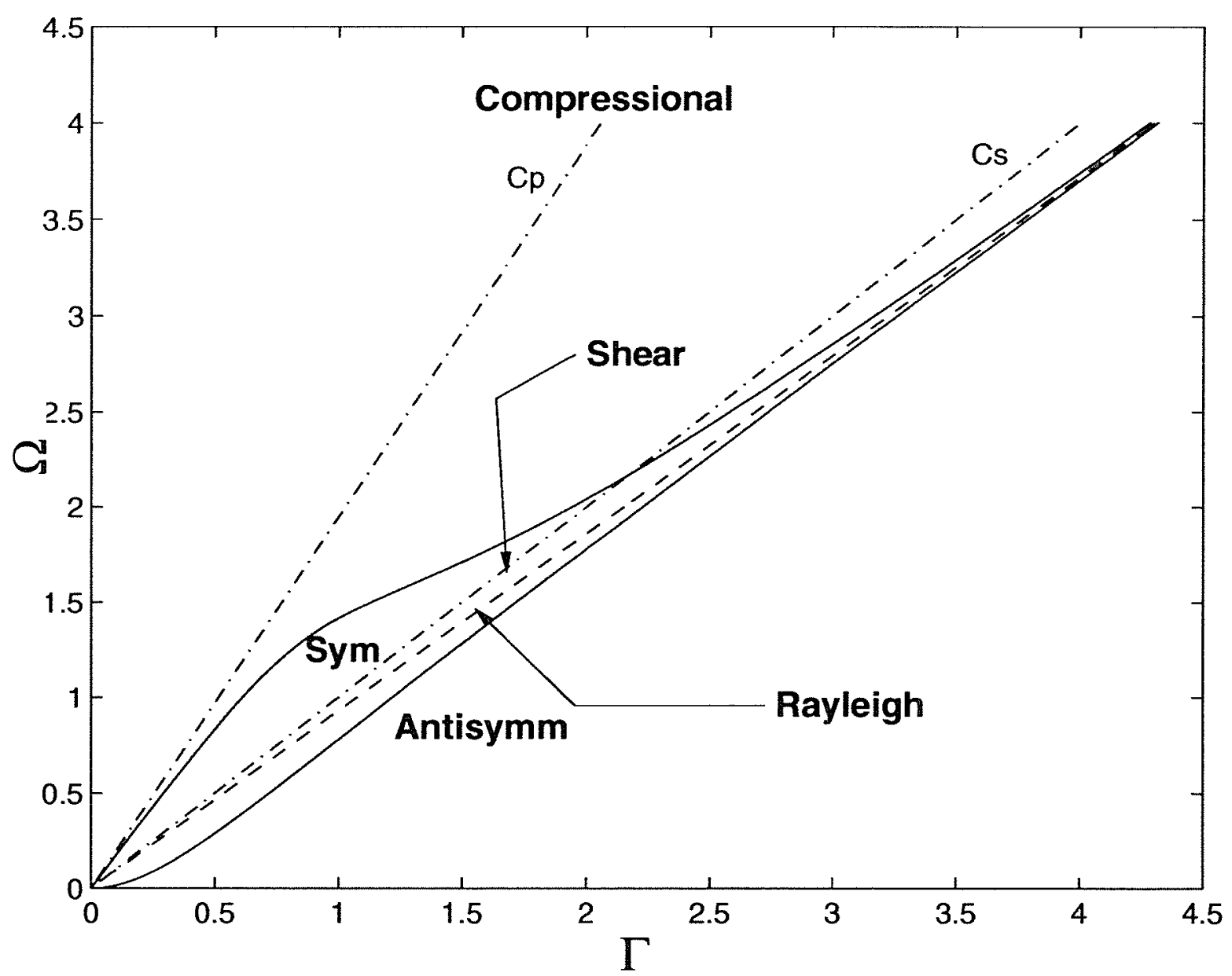

Figure 4-8: Dispersion curves of the first two fundamental Lamb modes for $c_{p}=$ $3500 \mathrm{~m} / \mathrm{s}, c_{s}=1800 \mathrm{~m} / \mathrm{s}$ given in non-dimensional form as non-dimensional frequency $\Omega$ versus non-dimensional horizontal wavenumber $\Gamma$ (two solid lines). Here $c_{p}=$ dispersion for compressional wave, $c_{s}=$ dispersion for shear wave, dashed line $=$ dispersion for the Rayleigh wave. 
These give the following depth dependencies

$$
\begin{array}{ll}
S_{A}^{+}=0 & S_{A}^{-}=0 \\
S_{B}^{+}=2 B & S_{B}^{-}=0 \\
S_{C}^{+}=0 & S_{C}^{-}=0
\end{array}
$$

The above depth dependencies give us the following expressions for the stress components which are used for the vertical boundary condition at $x=0$ :

$$
\begin{aligned}
\sigma_{x x} & =-4 \mu k_{x} k_{y} B \\
\sigma_{x y} & =2 \mu\left(k_{x}^{2}-k_{y}^{2}\right) B \\
\sigma_{x z} & =0
\end{aligned}
$$

So, the mode shape for the first symmetric Love mode is a constant. The dispersion relation takes the form

$$
\gamma \triangleq \mathcal{S}_{\mathrm{SH}}(\omega)=k_{s}
$$

\section{The first antisymmetric Love mode}

For the first symmetric Love mode we have the following symmetry conditions:

$$
\begin{aligned}
& A^{+}=A^{-}=0 \\
& B^{+}=-B^{-} \triangleq B \\
& C^{+}=C^{-}=0 .
\end{aligned}
$$


These give the following depth dependencies

$$
\begin{array}{ll}
S_{A}^{+}=0 & S_{A}^{-}=0 \\
S_{B}^{+}=2 B \sinh (\beta z) & S_{B}^{-}=-2 B \cosh (\beta z) \\
S_{C}^{+}=0 & S_{C}^{-}=0
\end{array}
$$

The above depth dependencies give us following expressions for the stress components which are used for the vertical boundary condition at $x=0$ :

$$
\begin{aligned}
& \sigma_{x x}=-4 \mu k_{x} k_{y} B \sinh (\beta z) \\
& \sigma_{x y}=2 \mu\left(k_{x}^{2}-k_{y}^{2}\right) B \sinh (\beta z) \\
& \sigma_{x z}=-2 i \mu k_{y} \beta B \cosh (\beta z)
\end{aligned}
$$

For the first antisymmetric Love mode the dispersion relation takes the form

$$
\sqrt{k_{s}^{2}-\gamma^{2}}=\frac{\pi}{2 h}
$$

We could solve it explicitly with respect to $\gamma$. As a result, we would obtain $\gamma$ as a function of frequency $\omega$ :

$$
\gamma \triangleq \mathcal{A}_{\mathrm{SH}}(\omega)=\sqrt{k_{s}^{2}-\frac{\pi^{2}}{4 h^{2}}}
$$

\subsubsection{The geometry of the problem}

The model geometry assumes a homogeneous elastic plate with two horizontal faces at $z= \pm h$, and a vertical edge at $x=0$ (Fig. 4-9). This geometry is obviously not separable, and does not allow for closed form solutions. However, several approximate solutions to similar plate vibration problems have been presented. 


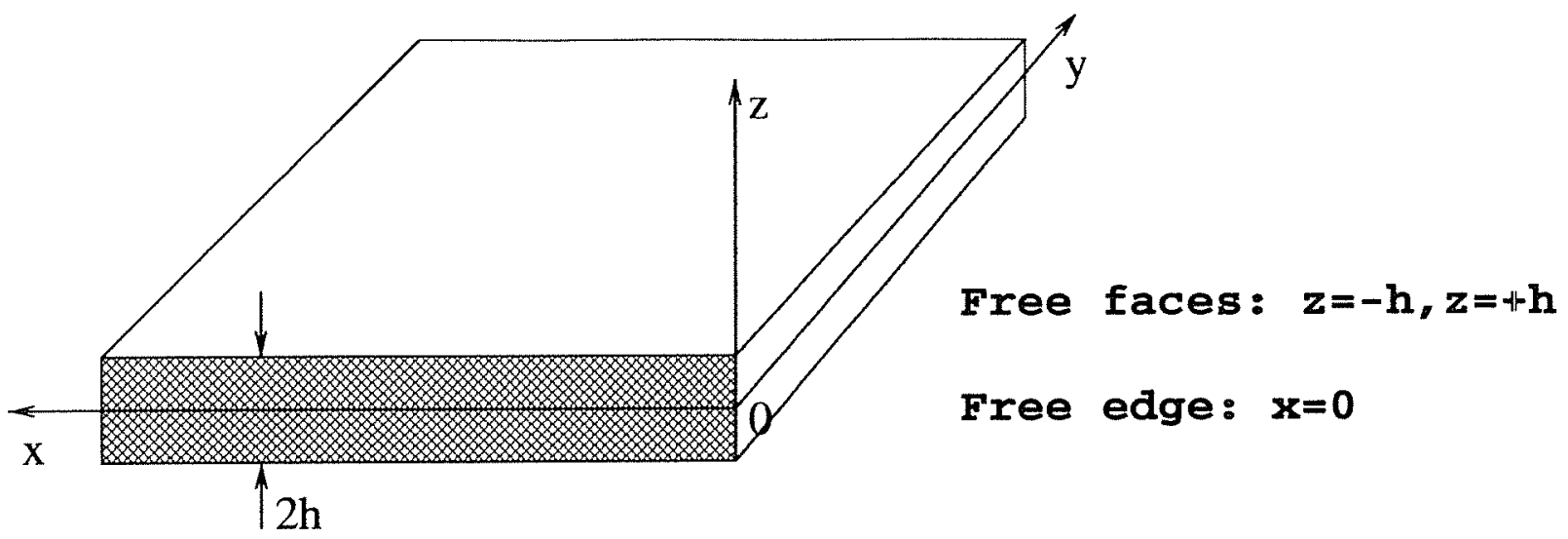

Figure 4-9: Edge wave model geometry. The ice plate is semi-infinite with horizontal faces at $z= \pm h$ and vertical edge at $x=0$. There is no edge at $y=0$.

\subsubsection{The boundary conditions in the weak form}

Mindlin [37] converts the 3-D equations of elasticity to an infinite series of 2-D equations by expanding the displacement in a power series of the thickness coordinate, and integrating through the plate thickness. This depth integration is conceptionally similar to the Galerkin approach used here. In the Galerkin approach [11], continuity of the field across the vertical boundary is expressed in the weak form:

$$
\begin{aligned}
& \int_{-h}^{h} \sigma_{z x} \mathrm{~d} z=0 \\
& \int_{-h}^{h} \sigma_{x x} \mathrm{~d} z=0 \\
& \int_{-h}^{h} \sigma_{x y} \mathrm{~d} z=0 .
\end{aligned}
$$

Basically, in this approach we minimize the least-square error in the vertical boundary condition across the plate thickness.

As a basis for the edge wave model the first symmetric and antisymmetric Lamb modes (P-SV) and first symmetric and antisymmetric Love modes (SH) of the infinite plate are assumed to be dominating. This is a reasonable assumption for frequencies below the thickness-shear frequency for the the ice cover, typically of the order $200-300 \mathrm{~Hz}$.

Here, we will consider the cases of different modes incident on the vertical 
interface of the plate. These modes will be the modes considered above. For all cases on the vertical interface we have in all waves interacting at the interface the identical values of $k_{y}$ and $k_{z}$ components of the wavenumber vector due to the trace matching condition. We will consider the $y$-component of the wavenumber vector as independent variable (denoted further as $\kappa$ ) and try to express the other components in terms of $\kappa$ and the medium wavenumbers. Note, the incidence is considered to be in the positive $x$-direction, and without fluid loading the plate geometry is symmetric, and the symmetric and antisymmetric equations are uncoupled.

\section{The incidence of the first symmetric Lamb mode}

Due to the symmetry of the problem we will have the following waves reflected from the vertical interface as a result of the incidence on it of the first symmetric Lamb mode (further referred to as $S_{I}$ ): reflected first symmetric Lamb mode (further referred to as $S_{R}$ ), reflected first symmetric Love mode (further referred to as $\mathrm{SH}_{R}^{(\mathrm{sym})}$ ). The corresponding wavenumber vectors will look like

$$
\begin{array}{ll}
S_{I}: & \mathbf{k}=\left(k_{1}, \kappa, k_{z}\right) \\
S_{R}: & \mathbf{k}=\left(-k_{1}, \kappa, k_{z}\right) \\
\mathrm{SH}_{R}^{\text {(sym) }}: & \mathbf{k}=\left(-k_{2}, \kappa, k_{z}\right)
\end{array}
$$

The horizontal wavenumber $\gamma$ should be determined from the corresponding dispersion relation for the first symmetric Lamb mode (see Equation (4.56)):

$$
\gamma=\mathcal{D}_{S}(\omega)
$$

We can now determine the $x$-component $k_{1}$ of the wavenumber vector in the incident wave from the value of the horizontal wavenumber and $\kappa$ (see above):

$$
k_{1}=\sqrt{\gamma^{2}-\kappa^{2}}
$$


The vertical component of the wavenumber vector could be obtained as

$$
k_{z}=\sqrt{k_{p}^{2}-\gamma^{2}}
$$

After that we can easily determine the value of $k_{2}$

$$
k_{2}=\sqrt{k_{s}^{2}-\left(k_{z}^{2}+\kappa^{2}\right)}=\sqrt{k_{s}^{2}-k_{p}^{2}+\gamma^{2}-\kappa^{2}}
$$

\section{The incidence of the first antisymmetric Lamb mode}

Due to the symmetry of the problem we will have the following waves reflected from the vertical interface as a result of the incidence on it of the first antisymmetric Lamb mode (further referred to as $A_{I}$ ): reflected first antisymmetric Lamb mode (further referred to as $A_{R}$ ), reflected first antisymmetric Love mode (further referred to as

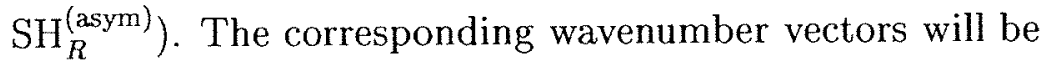

$$
\begin{array}{ll}
A_{I}: & \mathbf{k}=\left(k_{2}, \kappa, k_{z}\right) \\
A_{R}: & \mathbf{k}=\left(-k_{2}, \kappa, k_{z}\right) \\
\mathrm{SH}_{R}^{\text {(asym) }}: & \mathbf{k}=\left(-k_{2}, \kappa, k_{z}\right)
\end{array}
$$

The horizontal wavenumber $\gamma$ should be determined from the corresponding dispersion relation for the first antisymmetric Lamb mode (see Equation (4.61)):

$$
\gamma=\mathcal{D}_{A}(\omega)
$$

We can now determine the $x$-component $k_{2}$ of the wavenumber vector in the incident wave from the value of the horizontal wavenumber and $\kappa$ :

$$
k_{2}=\sqrt{\gamma^{2}-\kappa^{2}}
$$


The vertical component of the wavenumber vector could be obtained as

$$
k_{z}=\sqrt{k_{s}^{2}-\gamma^{2}} .
$$

After that we can easily determine the value of $k_{1}$ (though it is not really used in this case)

$$
k_{1}=\sqrt{k_{p}^{2}-\left(k_{z}^{2}+\kappa^{2}\right)}=\sqrt{k_{p}^{2}-k_{s}^{2}+\gamma^{2}-\kappa^{2}} .
$$

\section{The solution of the vertical problem}

Due to the simple character of the depth-dependence of the lowest order modes (hyperbolic functions) the depth integrals in Equations (4.71-4.73) could be evaluated in the closed form. These 'weak' boundary conditions then lead to a system of linear equations of the form

$$
\mathbf{G X}=\mathbf{Y}
$$

where $\mathrm{X}$ is the the wavefield amplitude vector: $\mathrm{X}=(A, B, C), \mathrm{Y}$ represents the contributions from an incident wave field.

The modal solutions are then found from the eigenvalue problem defined similarly to the homogeneous field case (compare with Equation (4.54)):

$$
\operatorname{det} \mathbf{G}=0 .
$$

So the procedure for the solution of the vertical problem can be outlined as follows:

- Choose the incident mode from the fundamental Lamb and Love modes of the infinite plate.

- For selected incident mode determine the components of wavenumber vector for all waves interacting at the vertical interface in this particular case.

- Calculate contributions to $\sigma_{z x}, \sigma_{x x}$ and $\sigma_{x y}$ from all the interacting waves after 
substituting the closed form expressions for the corresponding depth integrals.

- Combine all the terms corresponding to reflected waves on the left side of the system of linear equations representing 'weak' vertical boundary conditions (Equations (4.71-4.73)) to obtain matrix $\mathbf{G}$ and the reflected wavefield vector $\mathbf{X}$, while on the right side - all terms corresponding to the incident mode to obtain the vector $\mathbf{Y}$.

- Find the modal solutions to the problem by solving the equation

$$
\operatorname{det} \mathbf{G}=0
$$

with respect to $y$-component of the wavenumber vector $\kappa$.

\subsection{Comparison of model and the data}

The model results for mode dispersion have been compared to the results of the experimental analysis. The closest match was obtained for the antisymmetric problem, indicated by the solid line in Fig. 4-10. Between 10 and $20 \mathrm{~Hz}$ the theoretical prediction agrees reasonably well with the experimental values. On the other hand, the frequency dependence is obviously rather different for the model and the data, suggesting that a more complete model is required. The antisymmetric modes have significant vertical motion components, as is also evident in the experimental data, and it is therefore hypothesized that the fluid loading would significantly alter the dispersion relation. Thus, the added mass is expected to lower the phase velocity at the low frequency end, as required for improving the agreement in Fig. 4-10.

Additionally, polarization processing discussed in previous Chapter was carried out for the events involved in edge waves phenomenon. The results did not show clear picture for polarization of these events, though MPD HiV method gave somewhat lower standard deviation resulting in slight preferrence for SV-type of waves. One of the reasons for poor performance of polarization method in these conditions could be the very rough edge of ice lead in the vicinity of geophone arrays. The failure 


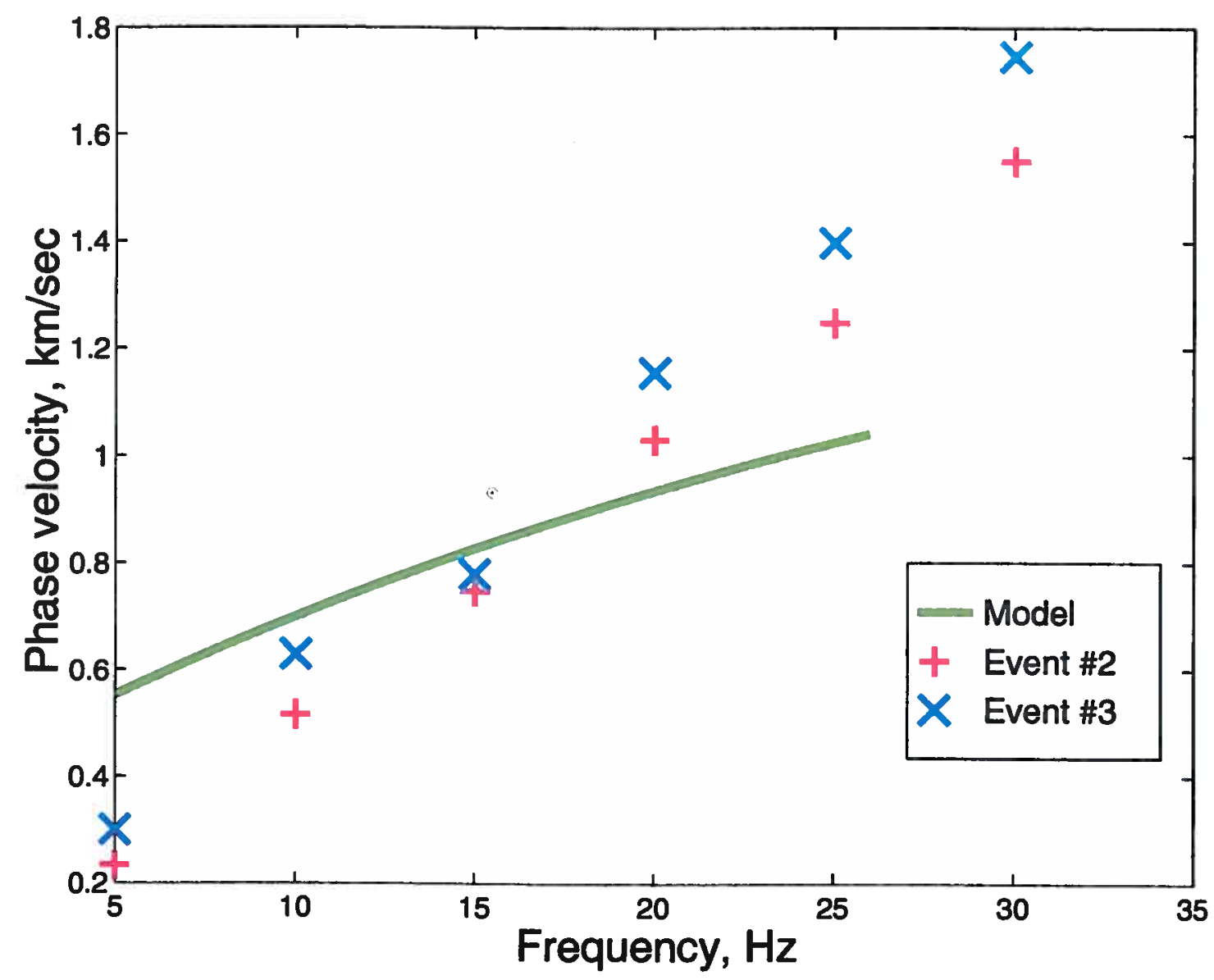

Figure 4-10: Comparison of model dispersion and the phase velocity estimates obtained by conventional beamforming of events \#2 and \#3.

of polarization processing for these events could also help to explain why the better agreement between theory and model for edge waves has not been achieved.

\subsection{Conclusions}

The described experiment [6] provided the evidence for the existence of edge waves, propagating along a newly opened lead. The waves exhibit a quasi-periodic behavior suggesting some kind of stick-slip generation mechanism somewhere along the length of the lead. The propagation characteristics of these waves were determined using seismic wavenumber estimation techniques. In the low-frequency limit the dispersion can be modeled approximately by an interaction at the lead edges of the lowest 
order, antisymmetric modes of the infinite plate. However, more modeling is required to understand the nature of this new 'edge wave' phenomenon, in particular in regard to achieving a better match of dispersion behavior. 


\section{Chapter 5}

\section{Event Identification}

\subsection{Overview}

An important component of ice mechanics processes, one which is critical to our understanding, is the type of fault motion during an ice event. The type of fault motion is defined by the relation between its direction of fault motion and some fixed coordinate directions. Each type of fault has its own unique radiation pattern which can be used to identify it. With multi-component data the differences between radiation patterns for several orthogonal components of motion also can be exploited.

This chapter starts by defining the fault orientation parameters and the three modes of ice cracking. The simulated radiation patterns for the three orthogonal components of motion in different types of ice cracks are then discussed, and simplified analytical models for ice crack radiation patterns are introduced. These analytical models are used in a procedure to identify events which is discussed in the next section. The procedure includes the determination of the directional characteristics of an ice event from the geophone array data.

The analytical models are fitted in turn to the event's directional characteristics; the best-fit model gives the most probable event mechanism (or type of fault motion in the event). In the next section the results of the determination of the event mechanisms for several groups of events are discussed. 


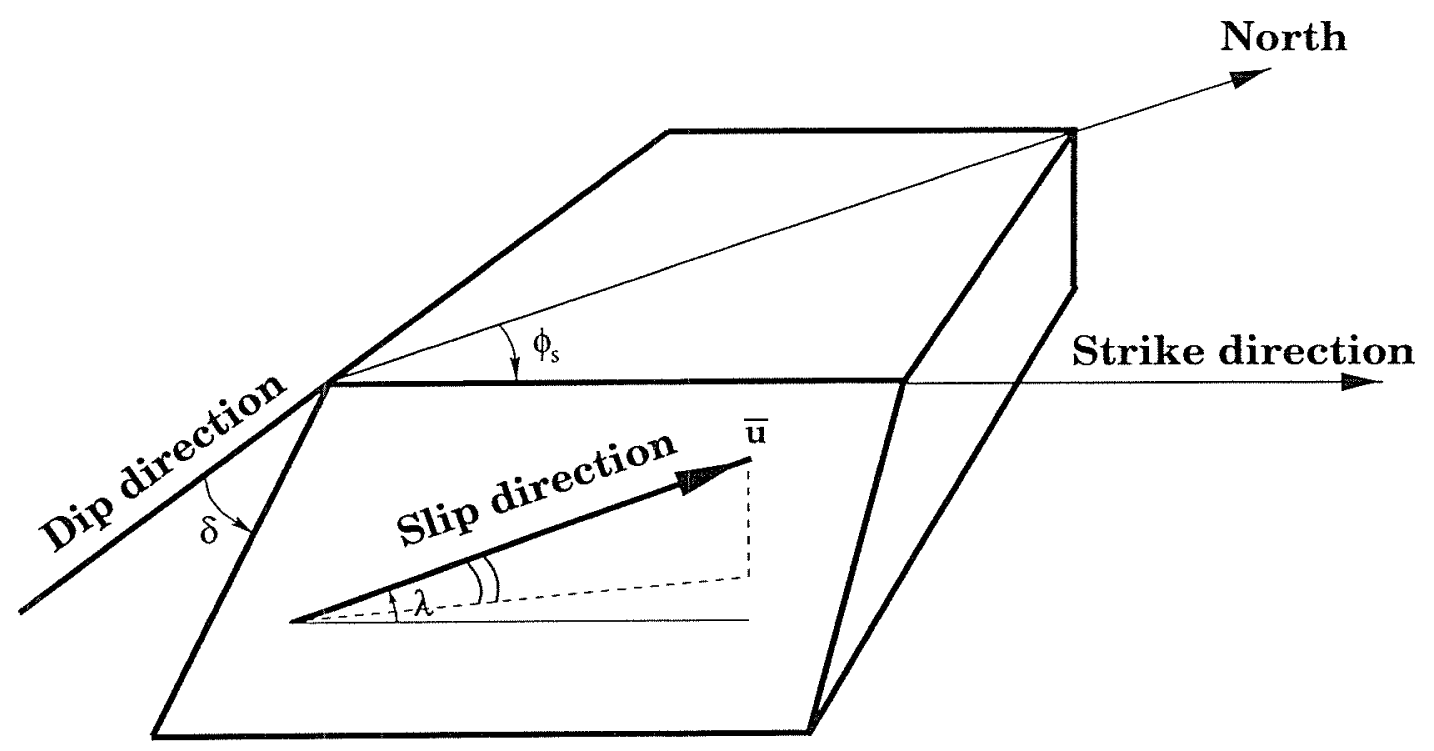

Figure 5-1: Definition of the fault-orientation parameters (strike $\phi_{s}$, dip $\delta$ ) and slipdirection (rake $\lambda$ ) according to [1].

\subsection{Fault types definitions}

\subsubsection{Fault orientation description}

The definitions of the fault-orientation parameters and slip, strike and dip directions are given according to $[1]$ (p. 106) on Fig. 5-1. On this figure the strike angle $\phi_{s}$ is measured clockwise from the north, with the fault dipping down to the right of the strike direction. Note that $0 \leq \phi_{s}<2 \pi$. The strike direction could also be defined as the direction along the intersection of the fault plane with the horizontal plane with

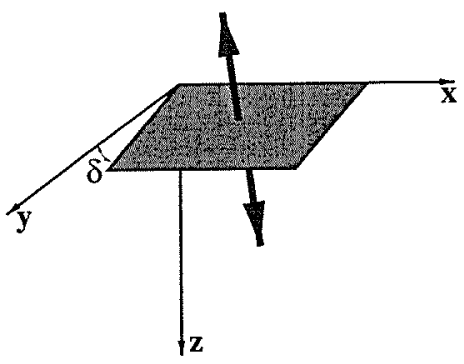

(a)

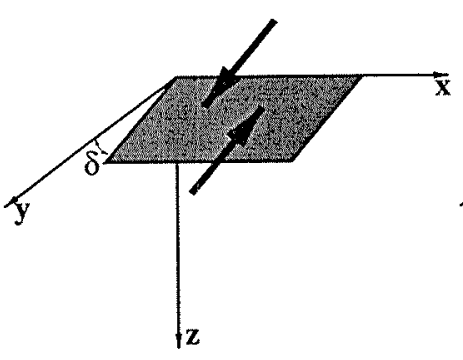

(b)

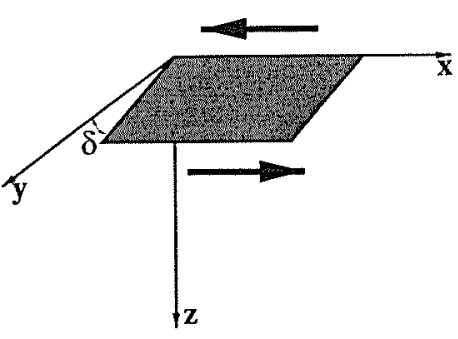

(c)

Figure 5-2: Fault motion with a dip angle $\delta$ for 3 different modes of cracking (according to [22]): (a) tensile crack, (b) dip-slip, (c) strike slip. On this plot the $x$-axis corresponds to direction to the North, $z$-axis - to the downward direction. 


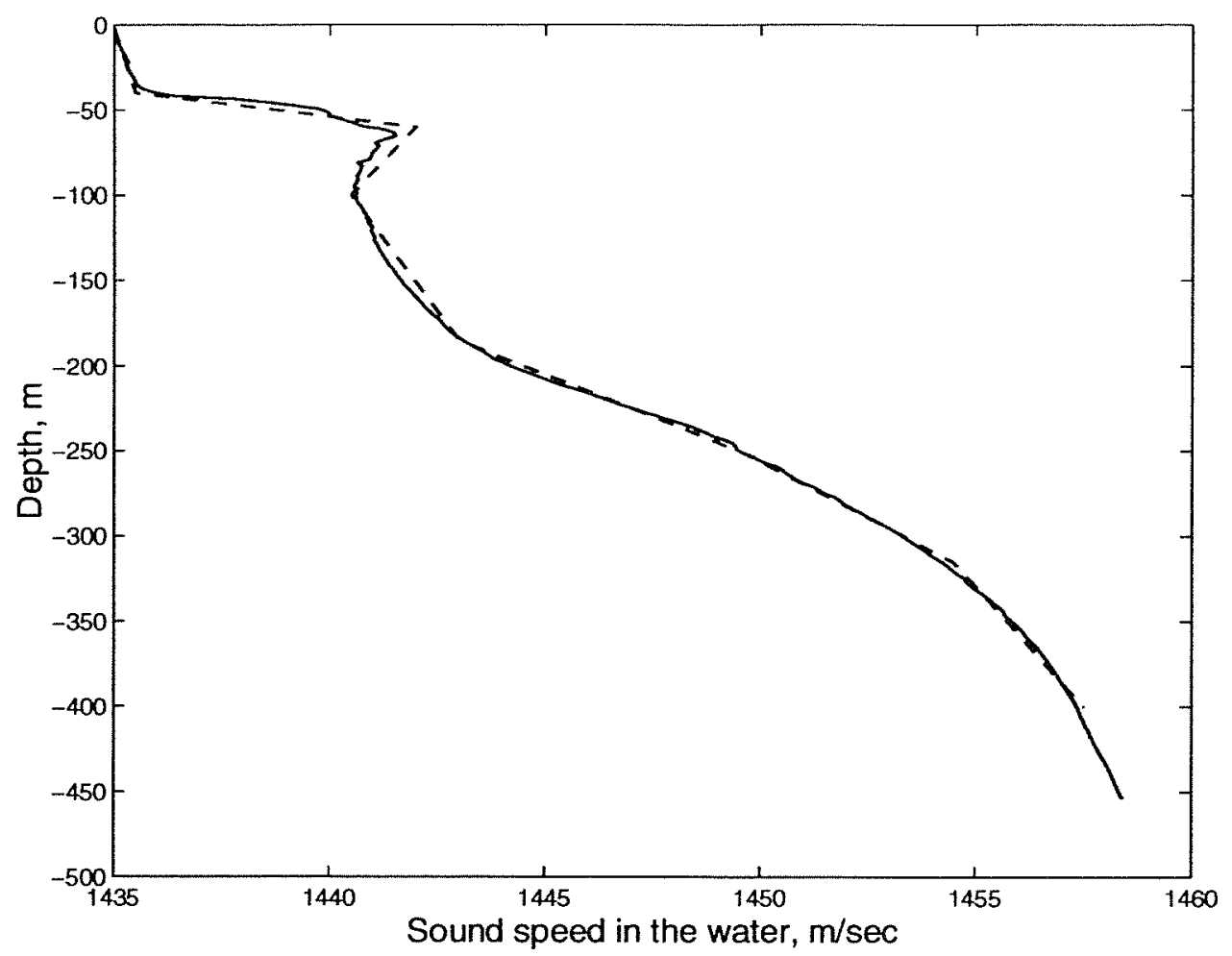

Figure 5-3: Sound profile in the water on April 22, 1994. Solid line shows the measured profile, the dashed one - the approximated one. The difference between two does not exceed $0.5 \mathrm{~m} / \mathrm{s}$ which, I believe, is less than time variability of the profile. Upper two meters of depth are occupied by the ice layer (compressional speed $-3500 \mathrm{~m} / \mathrm{sec}$ and shear wave speed $-1800 \mathrm{~m} / \mathrm{sec}$ ).

its sign chosen according to the above convention. The dip angle $\delta$ is measured down from the horizontal: $0 \leq \delta \leq 2 \pi$. It represents the angle between the fault plane and horizontal plane.

\subsubsection{Three modes of cracking}

The fault motion for 3 modes of cracking according to [22] is shown on Fig. 5-2. On this plot the $x$-axis points North, and the $z$-axis points down. Comparing with Fig. 5-1 we can say that strike-slip corresponds to a fault motion with rake angle $\lambda=0^{\circ}$ or $180^{\circ 1}$, and dip-slip to $\lambda=90^{\circ}$. The displacements are in the plane of crack and normal to the crack edge for dip-slip, and they are in the fault plane, parallel to

\footnotetext{
${ }^{1}$ left-lateral and right-lateral strike-slip faults respectively
} 
the leading edge of the crack in the strike-slip mode. These facts clarify the origin of the names "strike-slip" and "dip-slip" (one is in the strike direction, the other in the dip direction, according to Fig. 5-1). Note that in the seismology literature (see, for example, [1] p. 106) the above terms are reserved for cracks satisfying the additional condition that the dip angle is $\delta=90^{\circ}$. For the tensile crack the rake angle cannot be defined, because the particle displacement is normal to the fault surface (see Fig. 5-2a).

Sometimes tensile mode is also called Mode I or opening mode, dip-slip -Mode II (in-plane shear mode) or sliding mode, and strike-slip - Mode III (anti-plane shear) or tearing mode.

\subsection{Radiation patterns for different types of ice cracks}

The Arctic environment used for the calculation of radiation patterns for different types of events consisted of 10 layers. The first layer was a vacuum half-space, the second one was a homogeneous elastic ice of $2 \mathrm{~m}$ thickness (according to experimental records). The next 7 layers were water layers with a sound speed gradient for which analytical solutions could be obtained in the form of Airy functions. These water layers were obtained as an approximation to the measured sound speed profile on April 22, 1994 (see Fig. 5-3). The last layer was a fluid halfspace with constant sound speed because no information was available about the sea bottom and the contributions from the bottom on the energy in the ice could be neglected for all practical purposes.

The calculations of the radiation patterns for different types of ice events in the ice layers were made using the $O A S P 3 D$ module of the seismo-acoustic computational package $O A S E S$ created by $\mathrm{H}$. Schmidt [44]. For each event type the depth of the source and the receiver was assumed to be $0.2 \mathrm{~m}$. 


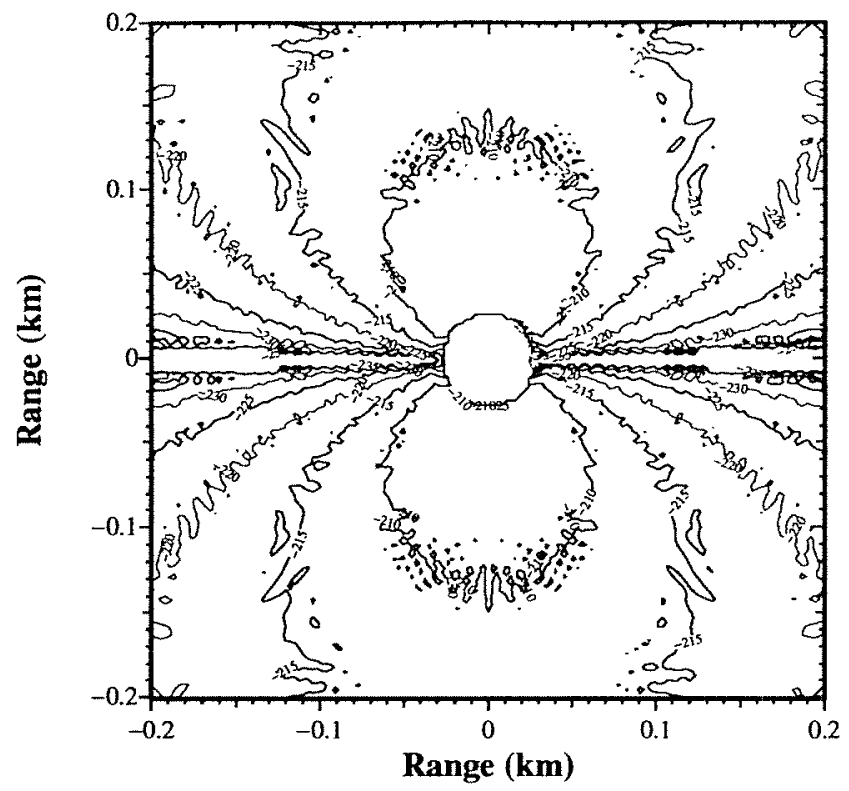

Figure 5-4: Radiation pattern for a dip-slip crack with a dip angle of $\delta=0^{\circ}$ in the near-field of the source at a frequency of $20 \mathrm{~Hz}$ : vertical component of velocity.

\subsubsection{Shear crack radiation patterns}

The horizontal radiation patterns for a dip-slip crack with a dip angle of $\delta=0^{\circ}$ are shown on Fig. 5-4 (vertical component of velocity), Fig. 5-5 (radial component of velocity), and Fig. 5-6 (transverse component of velocity). The radiation patterns for a dip-slip crack with a dip angle of $\delta=90^{\circ}$ are not shown because they are practically identical to those for a dip-slip crack with a dip angle of $\delta=0^{\circ}$. Three components of velocity in the near-field of the strike-slip crack with a dip-angle of $\delta=0^{\circ}$ are shown on Figs. 5-7, 5-8, and 5-9, respectively. The radiation patterns for a strike-slip crack with a dip-angle of $\delta=90^{\circ}$ are shown on Figs. 5-10, 5-11, and 5-12, respectively.

Note that for a dip-slip crack vertical and radial components of velocity have qualitatively identical radiation patterns, while the transverse component has a radiation pattern which could be obtained from the vertical radiation pattern by rotating it by $90^{\circ}$. The same observation also applies to a strike-slip crack with a dip-angle of $\delta=0^{\circ}$. For a strike-slip crack with a dip-angle of $\delta=90^{\circ}$, the respective rotation angle should be $45^{\circ}$. 


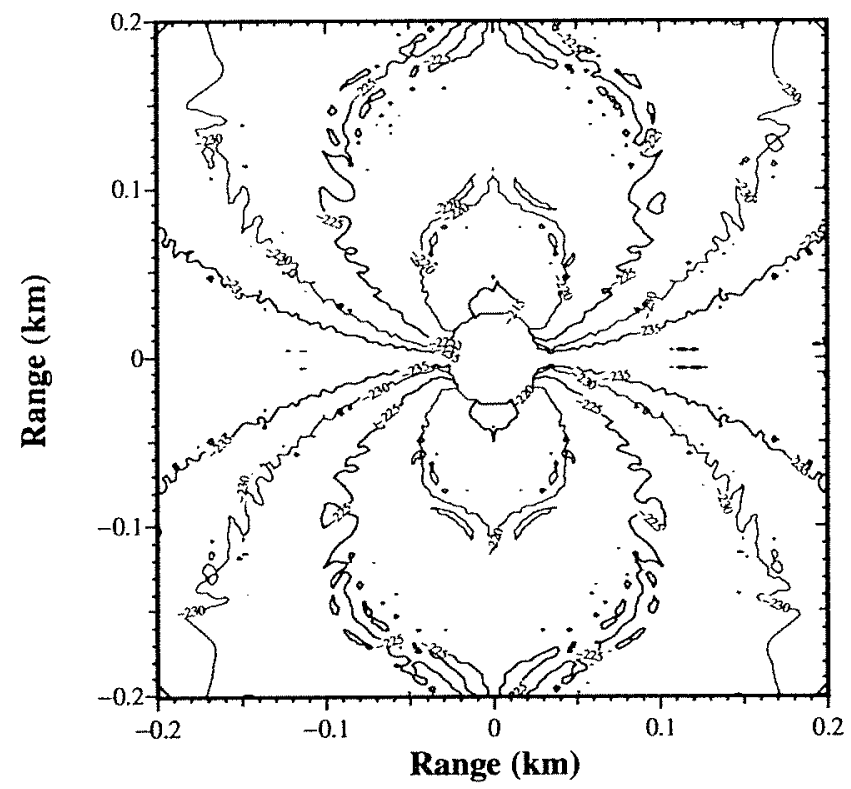

Figure 5-5: Radiation pattern for a dip-slip crack with a dip angle of $\delta=0^{\circ}$ in the near-field of the source at a frequency of $20 \mathrm{~Hz}$ : radial component of velocity.

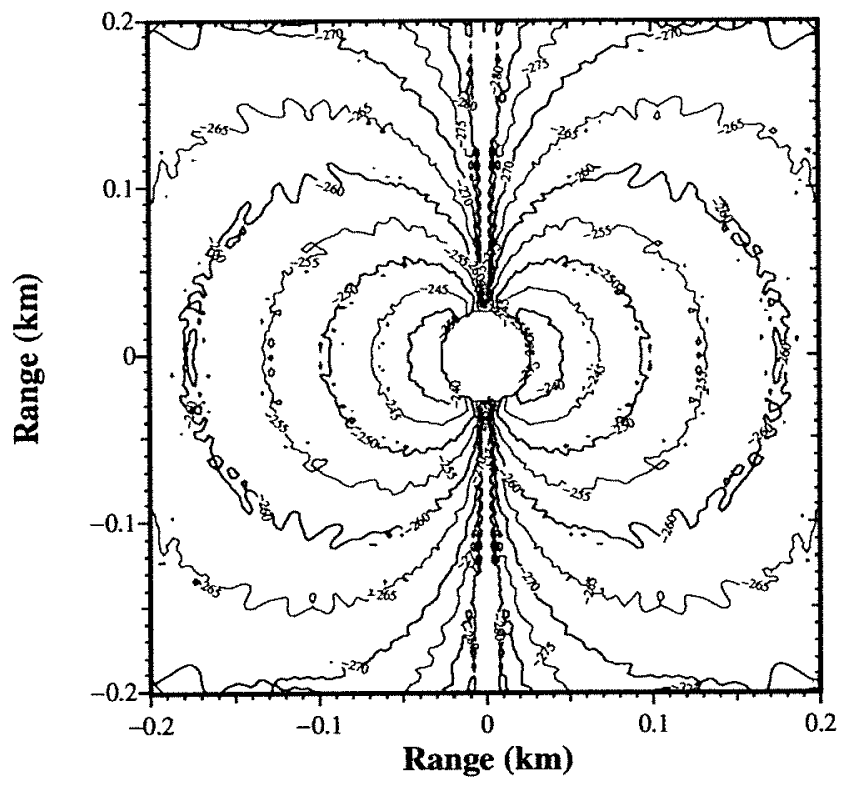

Figure 5-6: Radiation pattern for a dip-slip crack with a dip angle of $\delta=0^{\circ}$ in the near-field of the source at a frequency of $20 \mathrm{~Hz}$ : transverse component of velocity. 


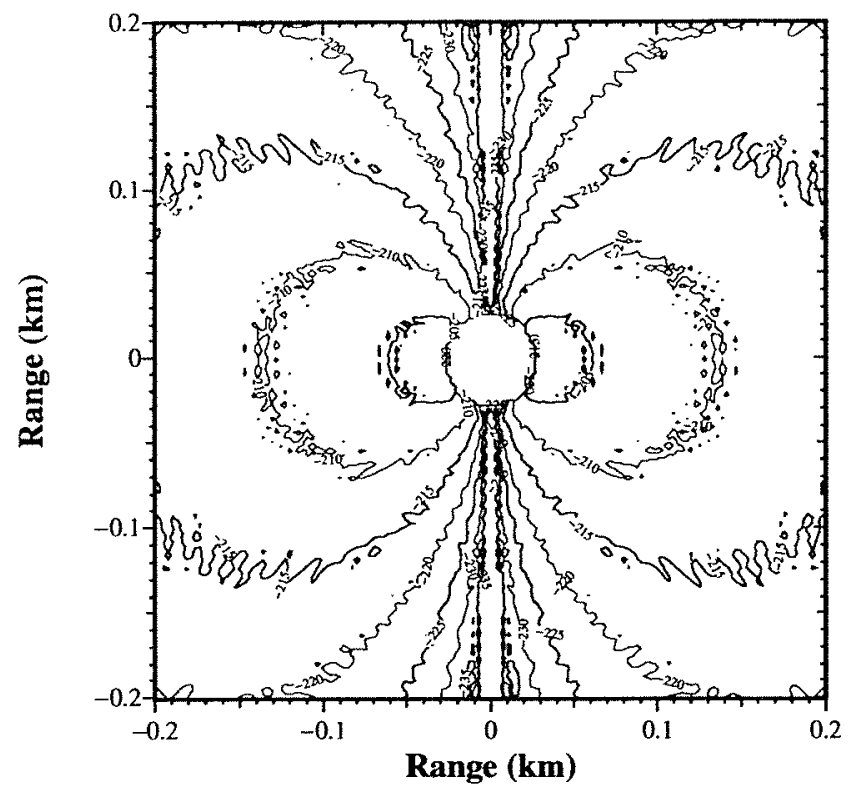

Figure 5-7: Radiation pattern for a strike-slip crack with a dip angle of $\delta=0^{\circ}$ in the near-field of the source at a frequency of $20 \mathrm{~Hz}$ : vertical component of velocity.

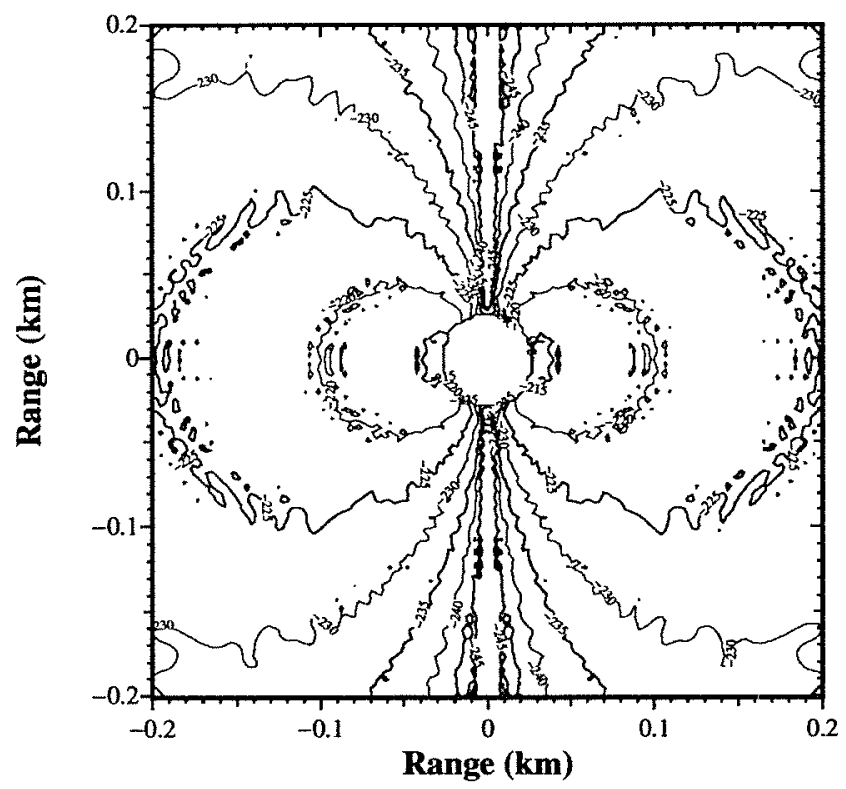

Figure 5-8: Radiation pattern for a strike-slip crack with a dip angle of $\delta=0^{\circ}$ in the near-field of the source at a frequency of $20 \mathrm{~Hz}$ : radial component of velocity. 


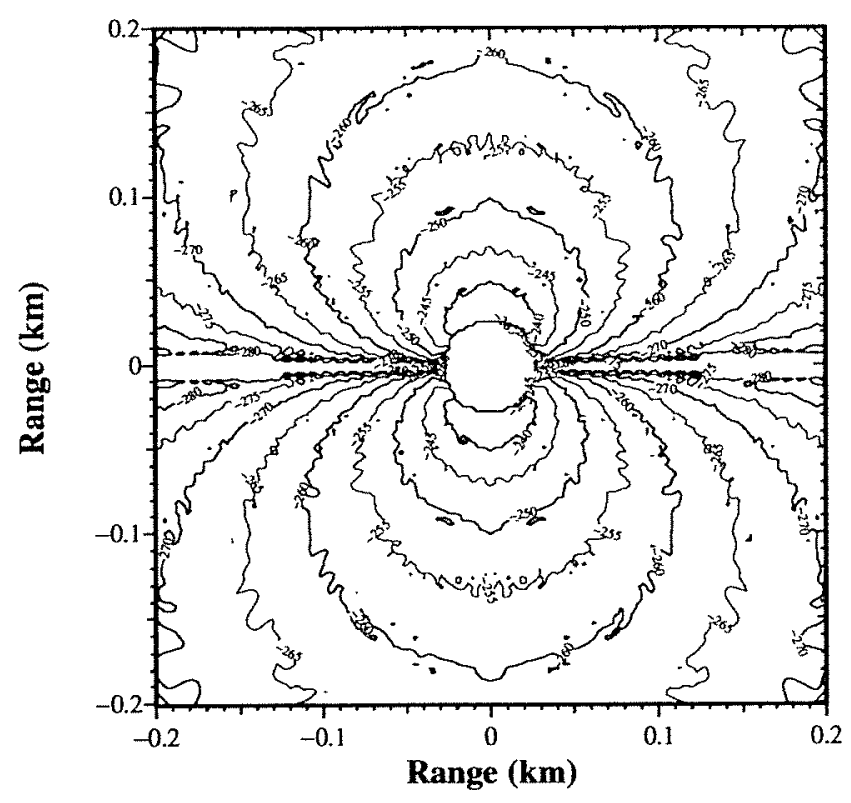

Figure 5-9: Radiation pattern for a strike-slip crack with a dip angle of $\delta=0^{\circ}$ in the near-field of the source at a frequency of $20 \mathrm{~Hz}$ : transverse component of velocity.

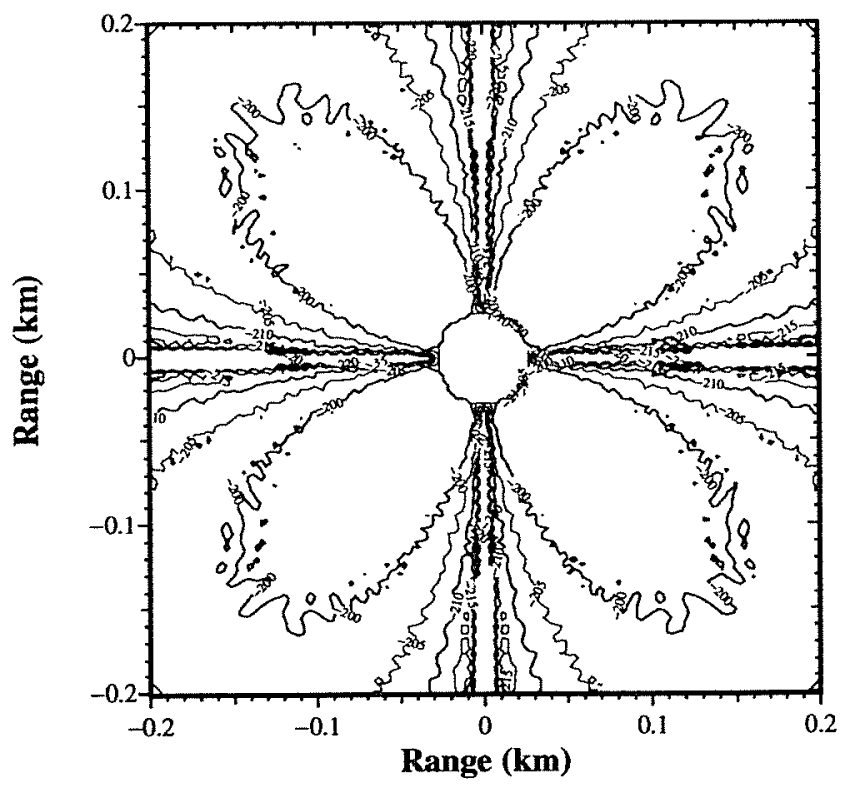

Figure 5-10: Radiation pattern for a strike-slip crack with a dip angle of $\delta=90^{\circ}$ in the near-field of the source at a frequency of $20 \mathrm{~Hz}$ : vertical component of velocity. 


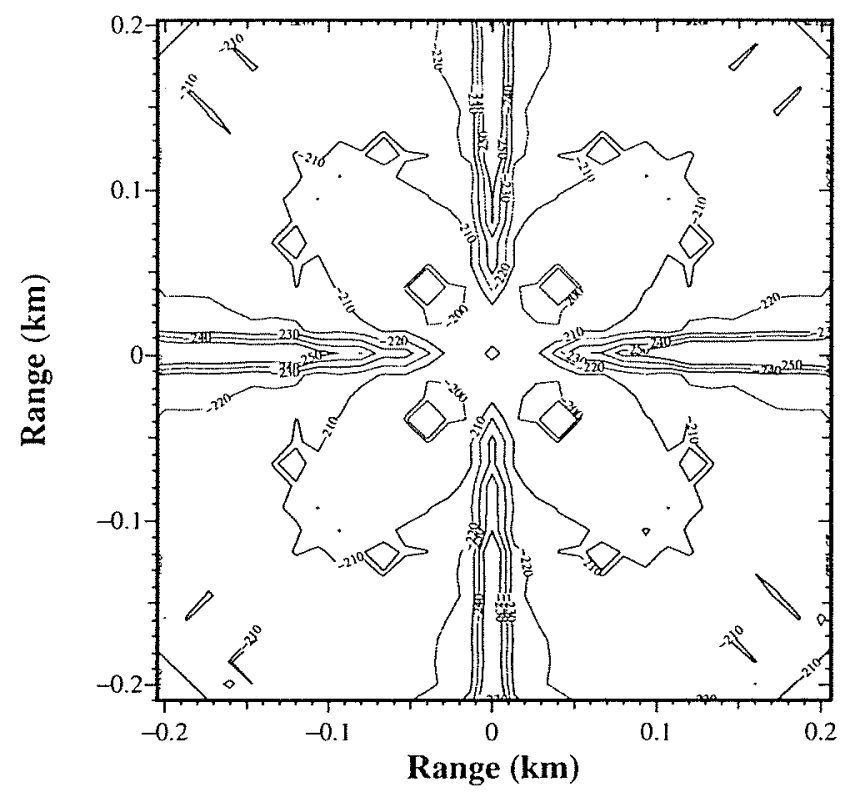

Figure 5-11: Radiation pattern for a strike-slip crack with a dip angle of $\delta=90^{\circ}$ in the near-field of the source at a frequency of $20 \mathrm{~Hz}$ : radial component of velocity.

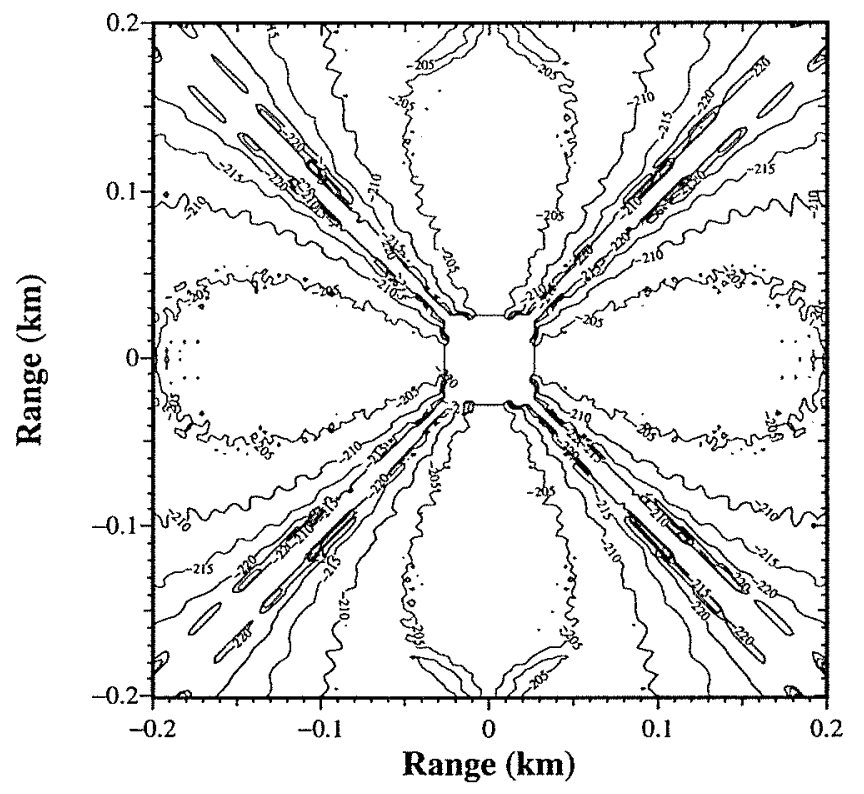

Figure 5-12: Radiation pattern for a strike-slip crack with a dip angle of $\delta=90^{\circ}$ in the near-field of the source at a frequency of $20 \mathrm{~Hz}$ : transverse component of velocity. 


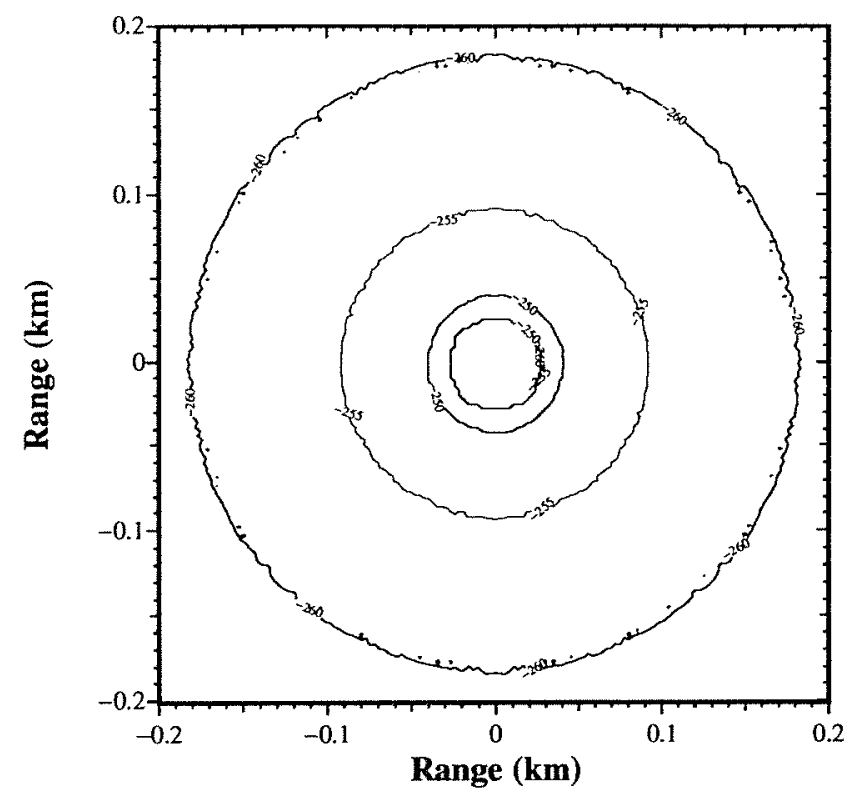

Figure 5-13: Radiation pattern for a tensile crack with a dip angle of $\delta=0^{\circ}$ in the near-field of the source at a frequency of $20 \mathrm{~Hz}$ : vertical component of velocity.

\subsubsection{Tensile crack radiation patterns}

For a tensile crack, experimentation with the values of the moment tensor components showed that the radiation pattern is insensitive to the relative values of these components for a fixed value of dip angle $\delta$. Calculations of radiation patterns for a tensile crack (for both values of dip-angle) were therefore carried out only for the case when the only non-zero component of the moment tensor is in the $X$-direction. For a dip-angle of $\delta=0^{\circ}$ the radiation of a tensile crack is omnidirectional, so only the vertical component of the ice velocity is shown (see Fig. 5-13). For a dip-angle of $\delta=90^{\circ}$, all three components of velocity are shown on Figs. 5-14, 5-15, and 5-16.

Note that while the vertical and radial components of velocity for a $\delta=90^{\circ}$ tensile crack have qualitatively identical radiation patterns, the transverse component has a quite distinct quadrupole radiation pattern resembling the vertical component of a strike-slip crack with a dip-angle of $\delta=90^{\circ}$. 


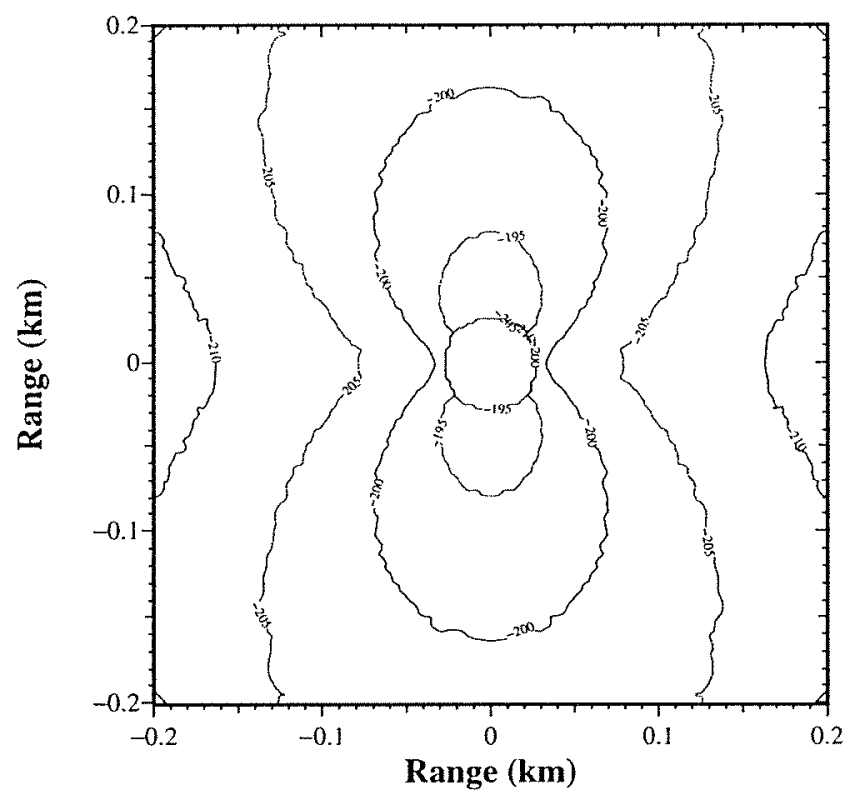

Figure 5-14: Radiation pattern for a tensile crack with a dip angle of $\delta=90^{\circ}$ in the near-field of the source at a frequency of $20 \mathrm{~Hz}$ : vertical component of velocity.

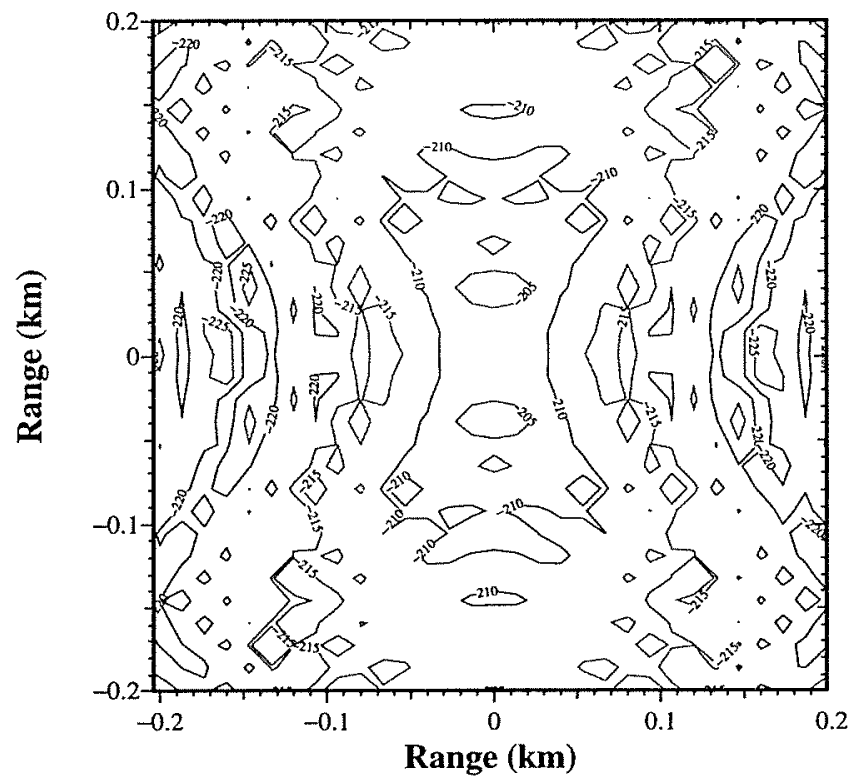

Figure 5-15: Radiation pattern for a tensile crack with a dip angle of $\delta=90^{\circ}$ in the near-field of the source at a frequency of $20 \mathrm{~Hz}$ : radial component of velocity. 


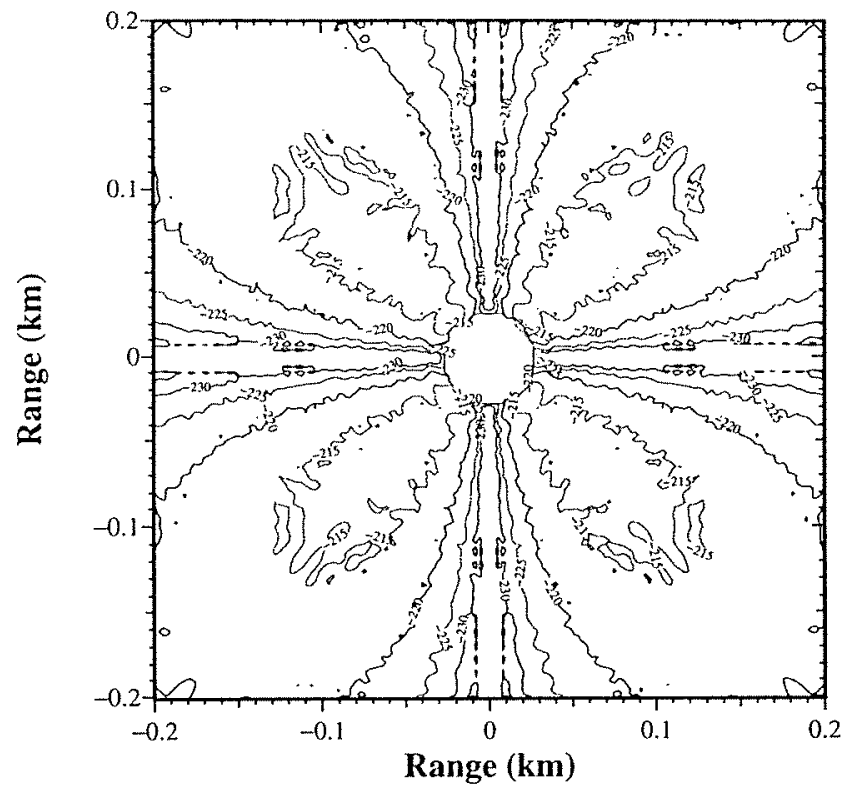

Figure 5-16: Radiation pattern for a tensile crack with a dip angle of $\delta=90^{\circ}$ in the near-field of the source at a frequency of $20 \mathrm{~Hz}$ : transverse component of velocity.

\subsubsection{Analytical models for crack radiation patterns}

The radiation patterns for different modes of cracking can be represented analytically by using three fundamental radiation patterns (monopole, dipole and quadrupole) and one radiation pattern obtained by the superposition of a monopole and a dipole (further called MD-type radiation pattern). As a result, a dip-slip crack with any dipangle and a strike-slip crack with a dip-angle of $\delta=0^{\circ}$ can be represented analytically as a dipole; a strike-slip crack with a dip-angle of $\delta=90^{\circ}$ corresponds to a quadrupole; a tensile crack with a dip-angle of $\delta=0^{\circ}$ radiates as a monopole source, while a tensile crack with a dip-angle of $\delta=90^{\circ}$ corresponds to a complex, MD-type analytical model of radiation pattern. Analytical expressions for these four models of radiation pattern are as follows:

$$
R= \begin{cases}\text { const } & \text { for monopole } \\ \sin ^{2} \varphi & \text { for dipole } \\ \sin 2 \varphi & \text { for quadrupole } \\ \frac{2}{3}-\frac{1}{3} \cos 2 \varphi & \text { for MD-type. }\end{cases}
$$




\begin{tabular}{|l||c|c|l|}
\hline Crack type & $\delta$ & Pattern type & Transverse component \\
\hline \multirow{2}{*}{ Dip-slip } & $0^{\circ}$ & Dipole & Rotated $90^{\circ}$ \\
\cline { 2 - 4 } & $90^{\circ}$ & Dipole & Rotated $90^{\circ}$ \\
\hline \multirow{2}{*}{ Strike-slip } & $0^{\circ}$ & Dipole & Rotated $90^{\circ}$ \\
\cline { 2 - 4 } & $90^{\circ}$ & Quadrupole & Rotated $45^{\circ}$ \\
\hline \multirow{2}{*}{ Tensile } & $0^{\circ}$ & Monopole & No rotation \\
\cline { 2 - 4 } & $90^{\circ}$ & MD-type & No rotation \\
\hline
\end{tabular}

Table 5.1: Correspondence between analytical models and crack types. The last column shows how the radiation pattern for the transverse component can be obtained from that of the radial or vertical component.

We summarize the correspondence between analytical models and crack types in the Table 5.1 .

\subsection{Procedure for fitting analytical radiation pat- terns to experimental data}

\subsubsection{Model parameterization}

The comparison between experimental data (relative signal levels on different geophones) and analytical radiation patterns has been made separately for each of the components of motion. The comparison has been carried out for levels in $\mathrm{dB}$ both for experimental data and radiation patterns. There is a slight difficulty here because, as one can see from Equation (5.1), some of the analytic patterns (dipole and quadrupole) have zeros which give rise to infinite levels in $\mathrm{dB}$. To avoid this problem one needs to specify the dynamic range of the radiation patterns beforehand. It is convenient to specify such a dynamic range from $0 \mathrm{~dB}$ to, say, $L_{\max } \mathrm{dB}$. In $L_{\max }$ we have a parameter of radiation pattern that could be changed to adjust the quality of fit between the analytical radiation pattern and experimental data. The other such parameter is the angle of rotation of the original radiation pattern given by Equation (5.1). It is obvious that this parameter is not useful for monopole-style radiation patterns due to the omnidirectional character of that pattern. For other 
radiation patterns to achieve rotation by $\varphi_{0}$ radians in the clockwise direction we need to substitute $\varphi \rightarrow \varphi+\varphi_{0}$ in Equation (5.1).

As a result, we have two parameters for adjusting the fit between the analytical radiation pattern and the experimentally determined signal levels on different geophones:

$L_{\max }-$ maximum level of radiation pattern in $\mathrm{dB}$;

$\varphi_{0}$ - angle of rotation of original analytical radiation pattern given by Equation (5.1). According to Fig. 5-1, $\varphi_{0}$ corresponds to the strike angle.

\subsubsection{Preprocessing of experimental data for determining the radiation patterns}

Now, let us consider experimental data. First, the event is located in space. Knowing its location, the respective direction to each geophone is easily determined. Denoting Cartesian coordinates of the event as $\left(x_{0}, y_{0}\right)$ and the $i$ th geophone - as $\left(x_{i}, y_{i}\right)$, we have the polar coordinates of the $i$ th geophone in the source coordinate system (centered at the event location):

$$
\begin{aligned}
R_{i} & =\sqrt{\left(x_{i}-x_{0}\right)^{2}+\left(y_{i}-y_{0}\right)^{2}}, \\
\varphi_{i} & =\arctan \frac{y_{i}-y_{0}}{x_{i}-x_{0}} .
\end{aligned}
$$

The next step is to determine the radiation level in the direction given by $\varphi_{i}$ from Equation (5.3). This level for each component of motion is determined as the peak intensity level in the considered arrival: $L_{i}$. As a result, for for each component of motion we have 5 points ${ }^{2}$ given by their polar coordinates $\left(L_{i}, \varphi_{i}\right)$ which determine (sometimes ambiguously) the radiation pattern of the event in question.

\footnotetext{
${ }^{2}$ Each RLAM unit had only 5 geophones
} 


\subsubsection{Model pattern fitting to experimental points}

Now, again for each component of motion separately, all available analytical models of radiation patterns (see Equation (5.1)) are fitted to the experimental data as specified above. For each particular model the best-fit $L_{\max }$ and $\varphi_{0}$ are found. The qualities of fit for different models are compared and the best-fit model $\mathcal{M}_{0}$ is chosen, having

corresponding best-fit parameters $L_{\max }^{\left(\mathcal{M}_{0}\right)}$ and $\varphi_{0}^{\left(\mathcal{M}_{0}\right)}$. The quality of fit for model $\mathcal{M}_{j}$ is determined by the standard deviation $\sigma_{\mathcal{M}_{j}}$ of the experimental points from the model radiation pattern. The deviation of individual experimental points from the analytical model is determined as a minimal distance from the experimental points to the curve representing the analytical model in polar coordinates. Due to the non-linear closed-form expressions for most analytical models (see Equation (5.1)) the least-square fit for individual experimental points (in order to find the minimal distance from the point to analytical curve) was carried out numerically by sampling the analytical curve in polar coordinates with sufficient angular resolution and finding the point in this discretized set with minimal distance to the experimental point in question.

In other words, we have a set of models $\mathcal{M}_{j} \in \mathbb{M}$ each parameterized by $L_{\max }$ and $\varphi_{0}$. The best-fit model can be expressed as

$$
\mathcal{M}_{0}=\arg \min _{\mathcal{M}_{j} \in \mathbb{M}} \sigma_{\mathcal{M}_{j}}
$$

The flow-chart for the model fit for each component of motion is shown on Fig. 5-17. Essentially, this procedure is a simple example of functional (or variational) analysis. The last stage of the fit process is to compare best-fit models for different components of motion. According to Table 5.1, we should get identical results (in terms of model and its best-fit parameters) for radial and vertical components, whereas for the transverse component, $\varphi_{0}$ should differ by $45^{\circ}$ for a strike-slip fault with a dip angle of $90^{\circ}$ and by $90^{\circ}$ for a strike-slip fault with a dip angle of $0^{\circ}$ and for a dip-slip fault with a dip angle of $0^{\circ}$ or $90^{\circ}$. If we observe the mentioned above differences in best-fit parameters for different components of motion (for, at least, two components), this 


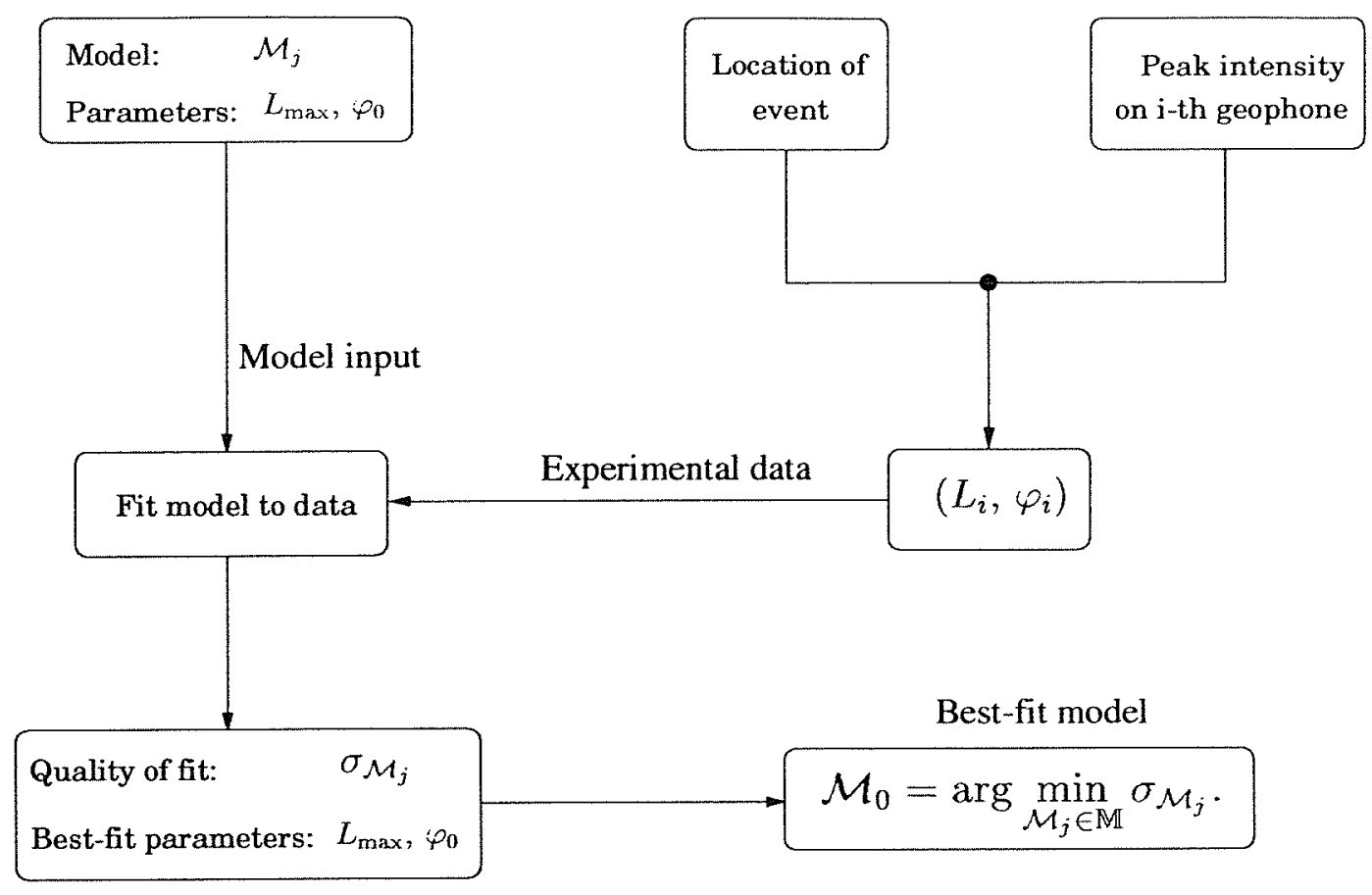

Figure 5-17: Flow-chart of the analytical radiation pattern fit for each component of motion.

should increase our confidence in the determination of the source mechanism.

\subsection{Qualitative determination of event mechanisms}

Using the above correspondence between crack types and the radiation pattern models, a qualitative determination of the source mechanisms of ice events was made for several groups of events. In the results discussed below the standard deviation of the best-fit model from the experimental points is presented on a linear scale as a fraction of the amplitude of the mainlobe of the model (expressed in \%). 


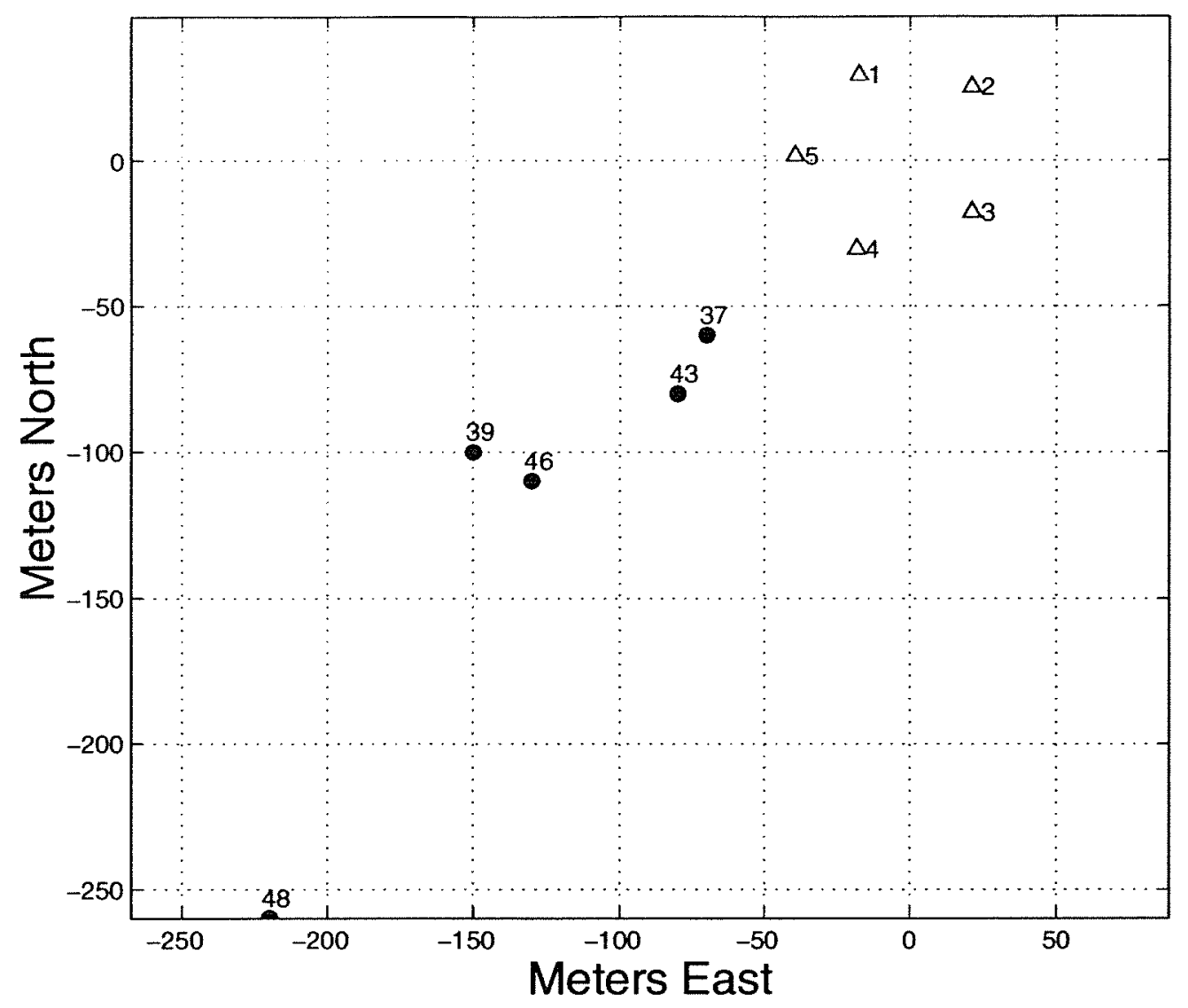

Figure 5-18: Locations of five events in Cluster \#1. Triangle markers are used to show the locations of geophones, while filled circles represent the locations of events. The numbers near the circle markers show approximate arrival times in seconds since the beginning of tape RLAM-26 for corresponding events.

\subsubsection{Cluster \#1}

The first group was Cluster \#1, a group of five events (for the locations of the events see Fig. 5-18) spanning approximately $10 \mathrm{sec}$ at the beginning of tape RLAM-26 with very similar time-domain behavior. The time series of the horizontal component of velocity for four events from that cluster are shown on Fig. 5-19. One can observe that for all four events three geophones (2-4) have a similar arrival pattern, while geophones \#1 and \#5 have quite different arrival patterns. The locations of the events for this cluster were determined by a standard least-square fit to the arrival times.

For event \#5 (approximately 39 sec from the beginning of tape RLAM-26) 

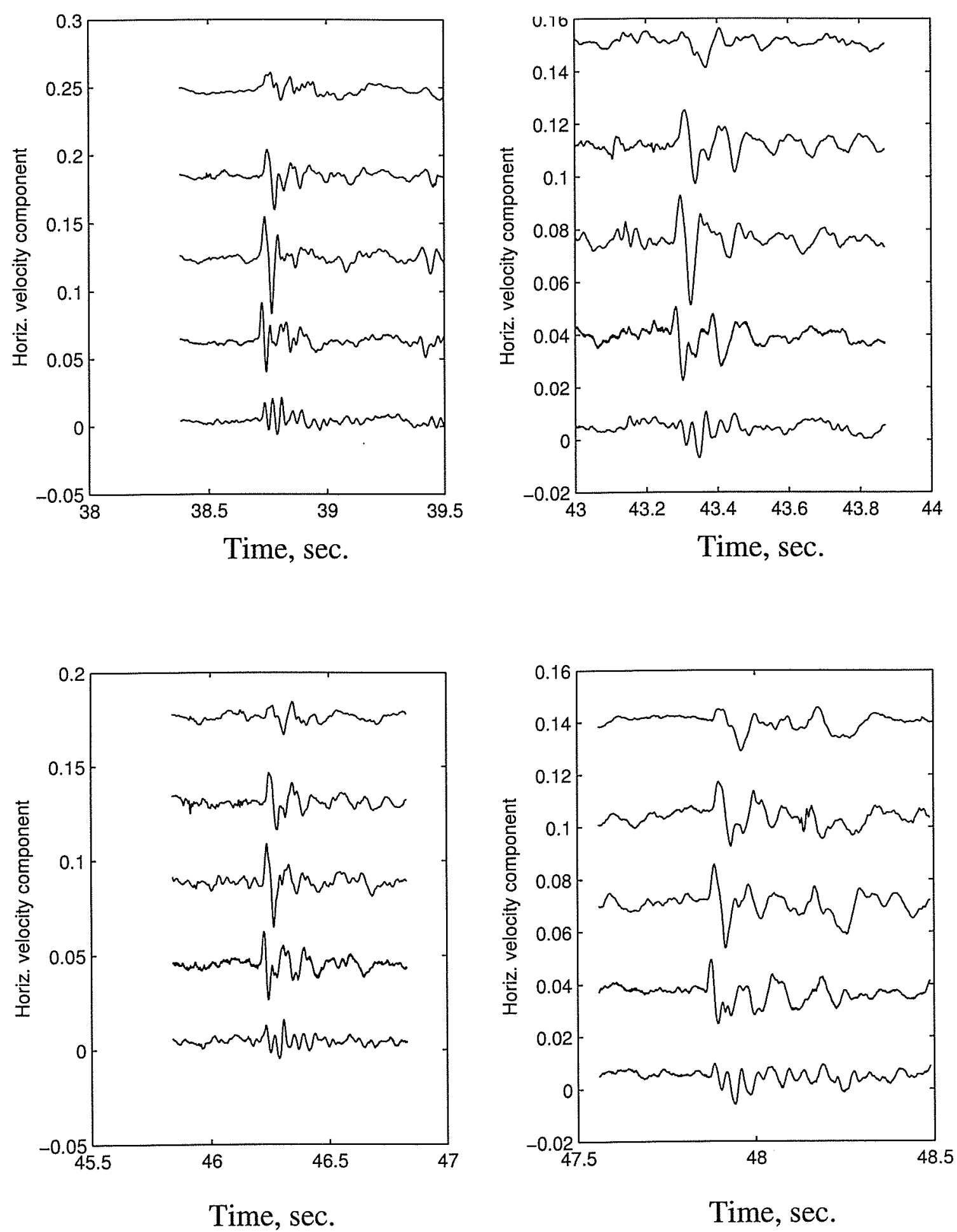

Figure 5-19: Stacked time series of horizontal component of velocity for four events in Cluster \#1. The top trace on all plots corresponds to geophone \#1, the bottom one to geophone \#5. 


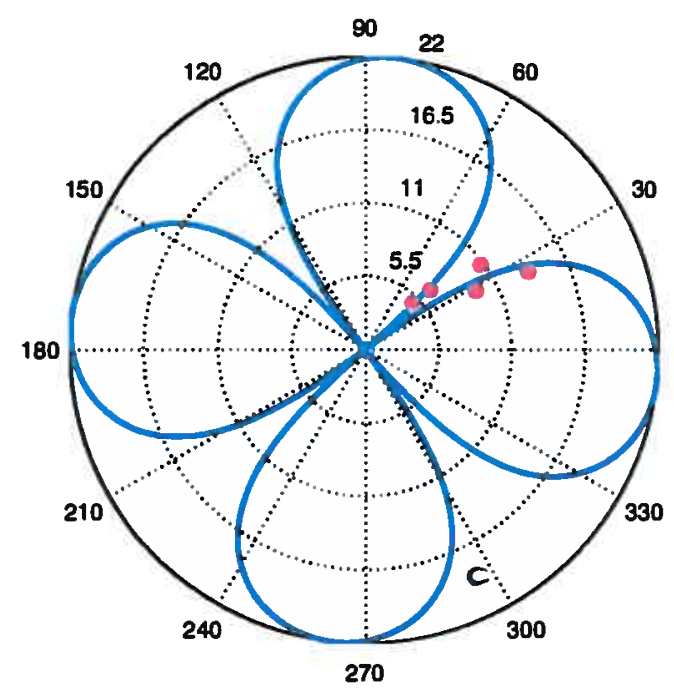

a)

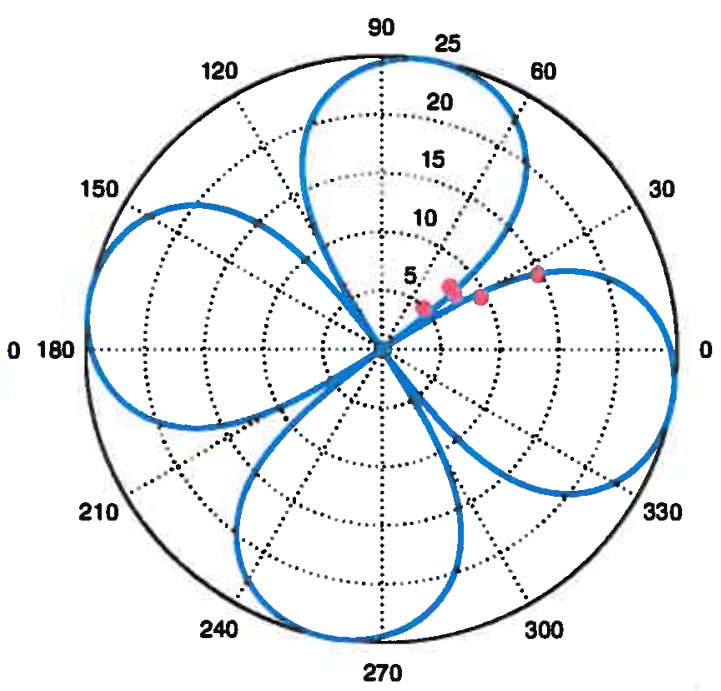

b)

Figure 5-20: Best-fit radiation pattern models for event \#5 from Cluster \#1 (approximately $39 \mathrm{sec}$ from the beginning of tape RLAM-26): (a) vertical component of motion, $\varphi_{0}=-39^{\circ}, \sigma_{\mathcal{M}}=4.5 \%$; (b) radial component of motion (to source), $\varphi_{0}=-35^{\circ}, \sigma_{\mathcal{M}}=3.1 \%$. Red circle markers correspond to experimental data from the five geophones.

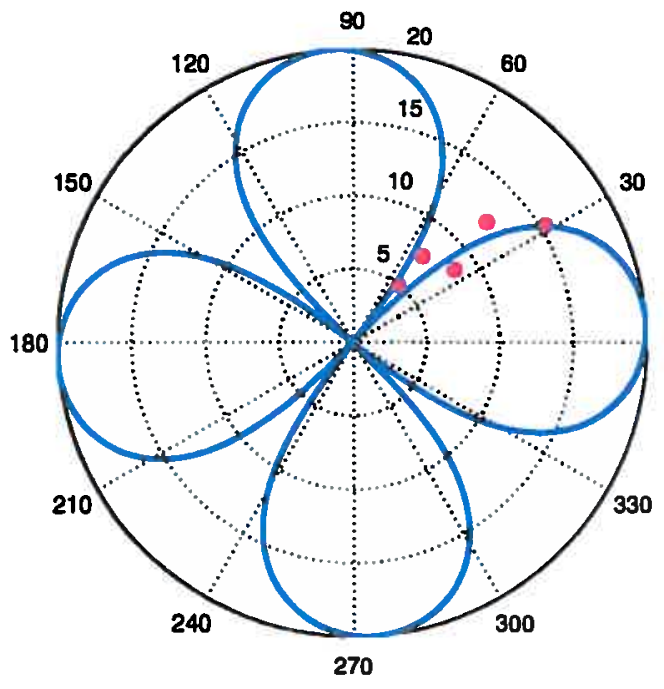

a)

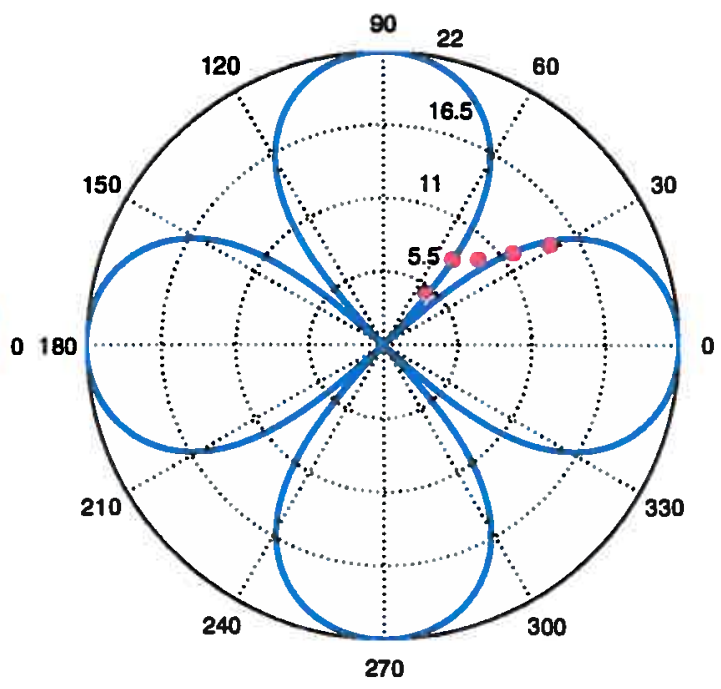

b)

Figure 5-21: Best-fit radiation pattern models for event \#7 from Cluster \#1 (approximately $46 \mathrm{sec}$ from the beginning of tape RLAM-26): (a) vertical component of motion, $\varphi_{0}=41^{\circ}, \sigma_{\mathcal{M}}=4.5 \%$; (b) radial component of motion (to source), $\varphi_{0}=45^{\circ}$, $\sigma_{\mathcal{M}}=2.4 \%$. Red circle markers correspond to experimental data from the five geophones. 
the above procedure yielded a quadrupole as the best-fit model, which corresponds to a strike-slip fault with a dip angle of $90^{\circ}$. In Fig. 5-20 the best-fit models are shown for the vertical and radial components. These models gave quite close values of strike angles $\left(-39^{\circ}\right.$ and $-35^{\circ}$, respectively). The fit for the transverse component in this case is not acceptable and therefore is not shown here. Similar results for event \#7 (approximately $46 \mathrm{sec}$ from the beginning of tape RLAM-26) are shown on Fig. 5-21. Again, the strike-slip fault mechanism (with a dip angle of $90^{\circ}$ ) is suggested for the event. The values of strike angles in this case are $41^{\circ}$ for the vertical component, and $45^{\circ}$ for the radial component. 


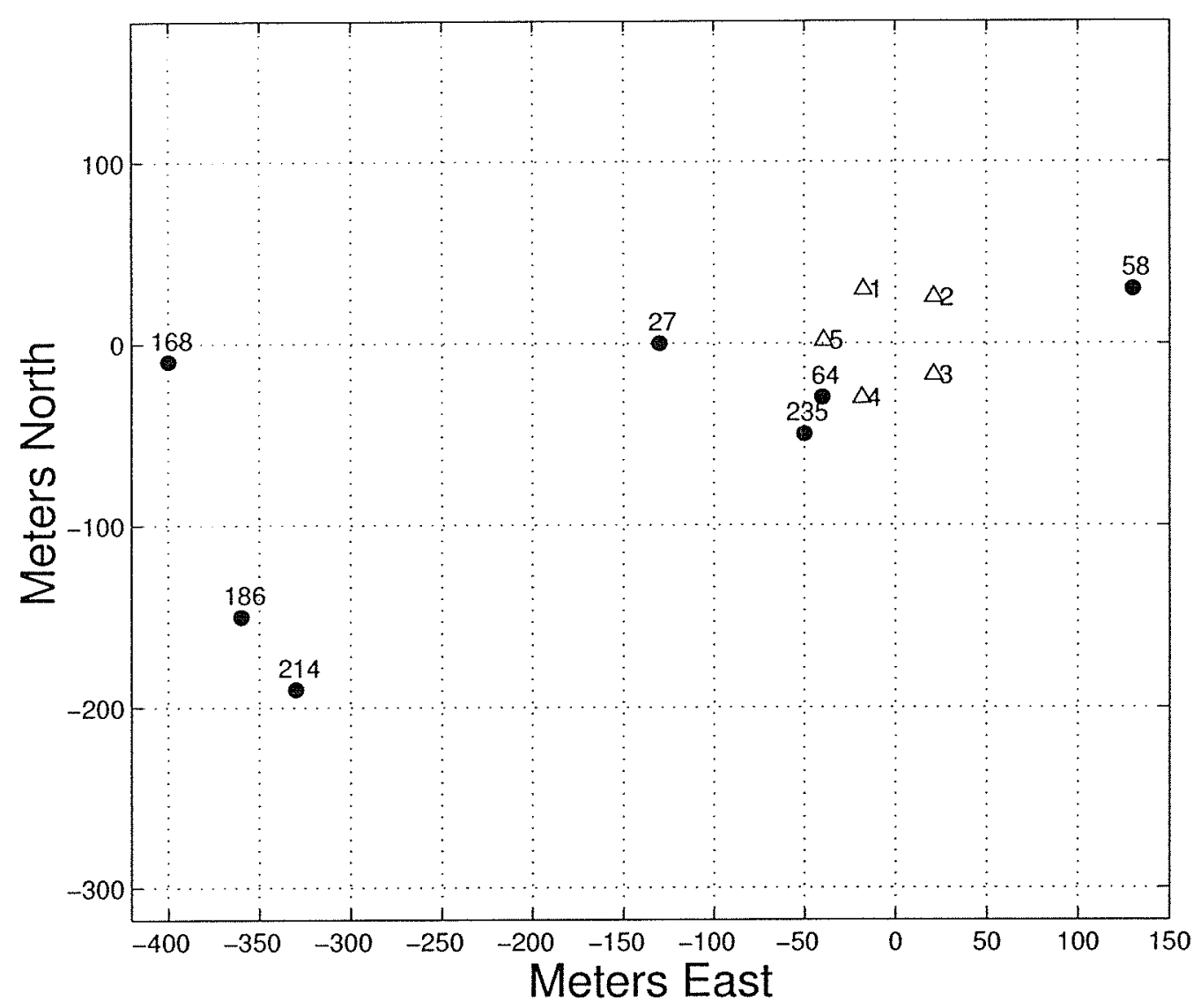

Figure 5-22: Locations of seven events at the beginning of tape RLAM-26. Triangle markers are used to show the locations of geophones, while filled circles represent the locations of events. The numbers near circle markers show approximate arrival times in seconds since the beginning of tape RLAM- 26 for corresponding events.

\subsubsection{Other events at the beginning of tape RLAM-26}

Several other events at the beginning of tape RLAM-26 were also successfully located by standard least-square fit to arrival times. Their locations are shown on Fig. 5-22.

For these events, an especially good fit was obtained for event \#10 (approximately $64 \mathrm{sec}$ from the beginning of tape RLAM-26). The discrimination between different models was also outstanding. The best fit was achieved for a quadrupole radiation pattern, corresponding to a strike-slip fault with a dip angle of $90^{\circ}$. On Fig. 5-23 the best-fit models are shown for the vertical and radial components. The quality of fit is better appreciated by comparing with fits by two other models (dipole and 


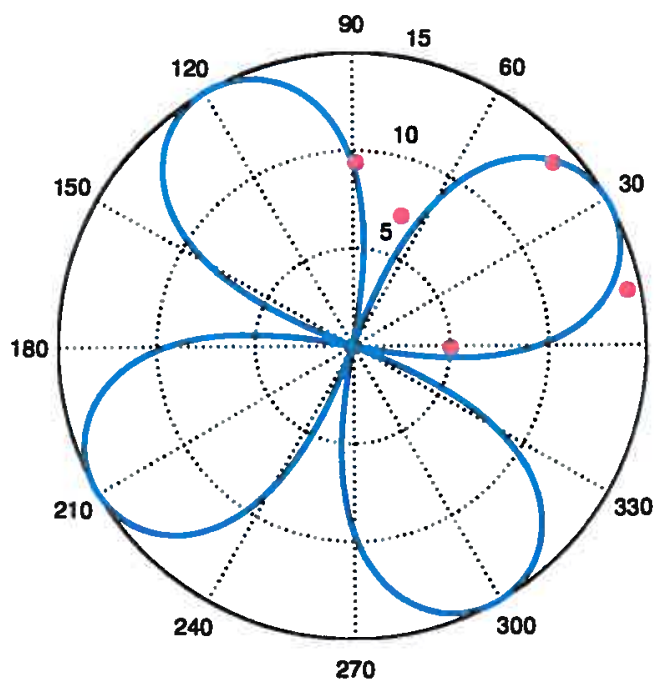

a)

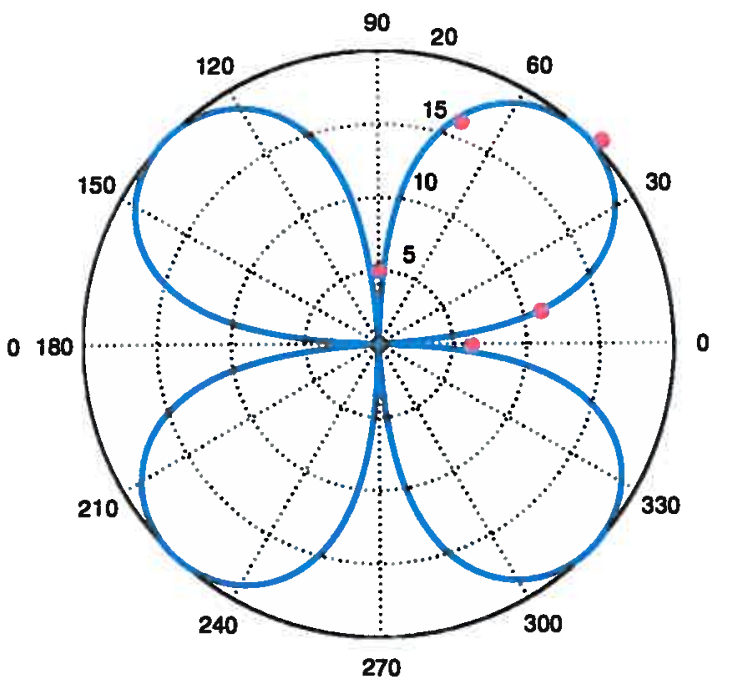

b)

Figure 5-23: Best-fit models of radiation patterns for event \#10 (approximately 64 sec from the beginning of tape RLAM-26): (a) vertical component of motion, $\varphi_{0}=16^{\circ}$, $\sigma_{\mathcal{M}}=4.4 \%$; (b) radial component of motion (to source), $\varphi_{0}=-1^{\circ}, \sigma_{\mathcal{M}}=2.1 \%$. Red circle markers correspond to experimental data from the five geophones.

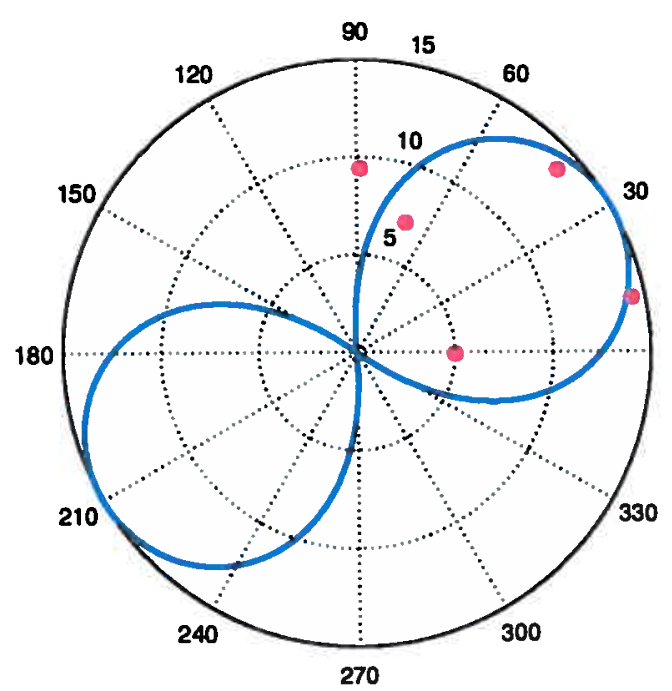

a)

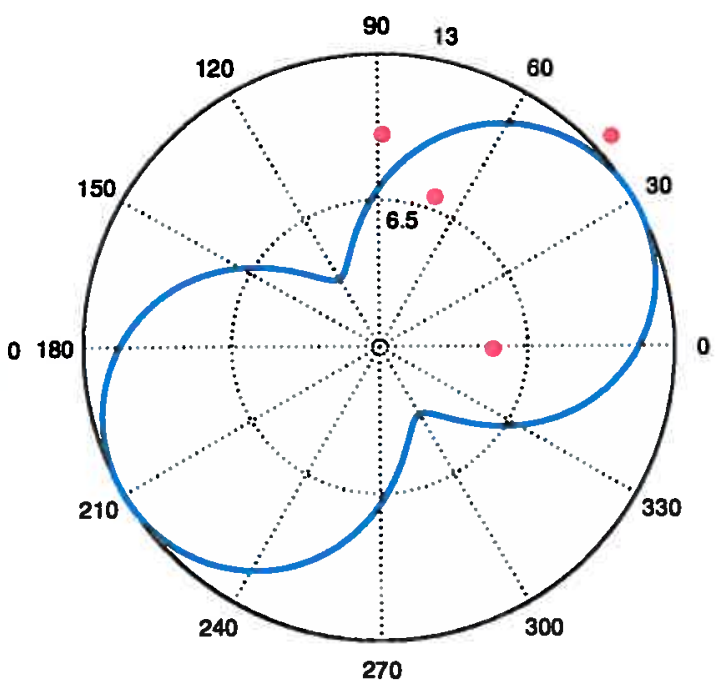

b)

Figure 5-24: Results of fits by two models (dipole and MD-type) for the vertical component of motion during event \#10 (approximately 64 sec from the beginning of tape RLAM-26): (a) dipole model, $\varphi_{0}=60^{\circ}, \sigma_{\mathcal{M}}=10.6 \%$; (b) MD-type model, $\varphi_{0}=60^{\circ}, \sigma_{\mathcal{M}}=15.0 \%$. Red circle markers correspond to experimental data from the five geophones. 


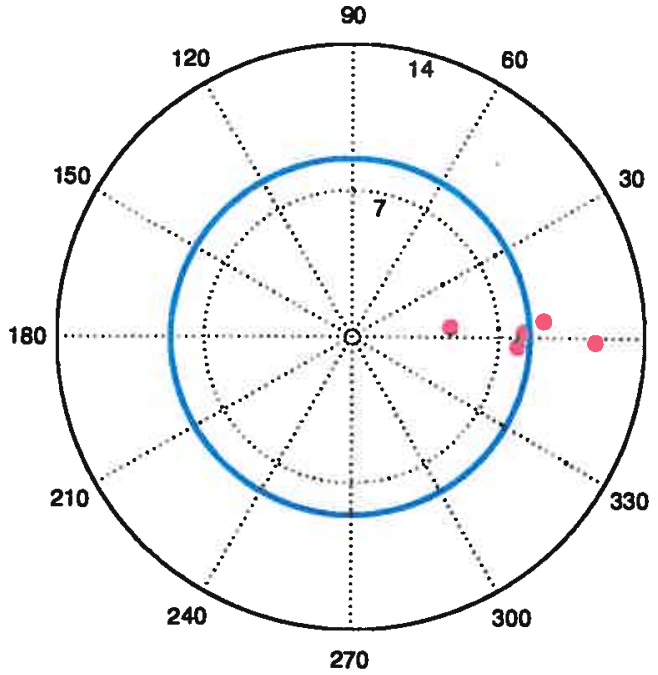

a)

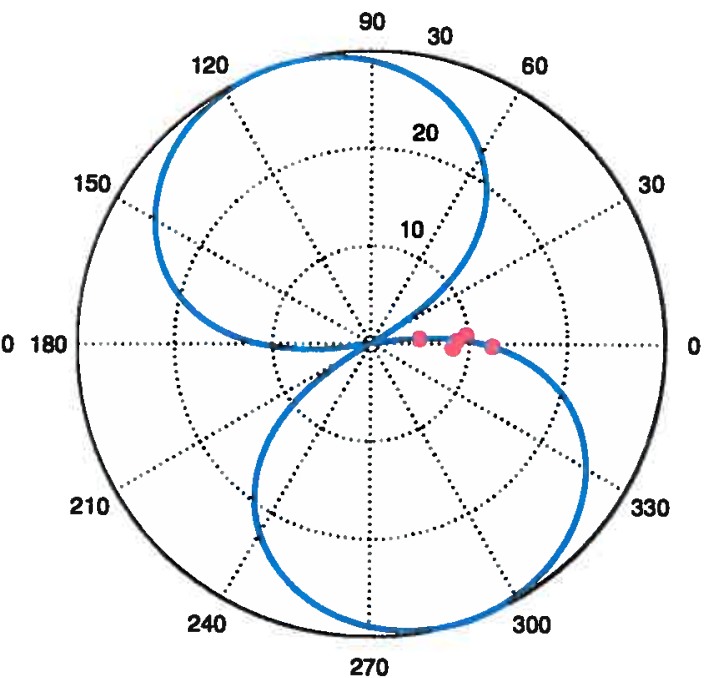

b)

Figure 5-25: Results of fits by two models (monopole and dipole) for the vertical component of motion during event \#16 (approximately $168 \mathrm{sec}$ from the beginning of tape RLAM-26): (a) monopole model, $\sigma_{\mathcal{M}}=19.2 \%$; (b) dipole model, $\varphi_{0}=$ $-20^{\circ}, \sigma_{\mathcal{M}}=4.1 \%$. Red circle markers correspond to experimental data from the five geophones.

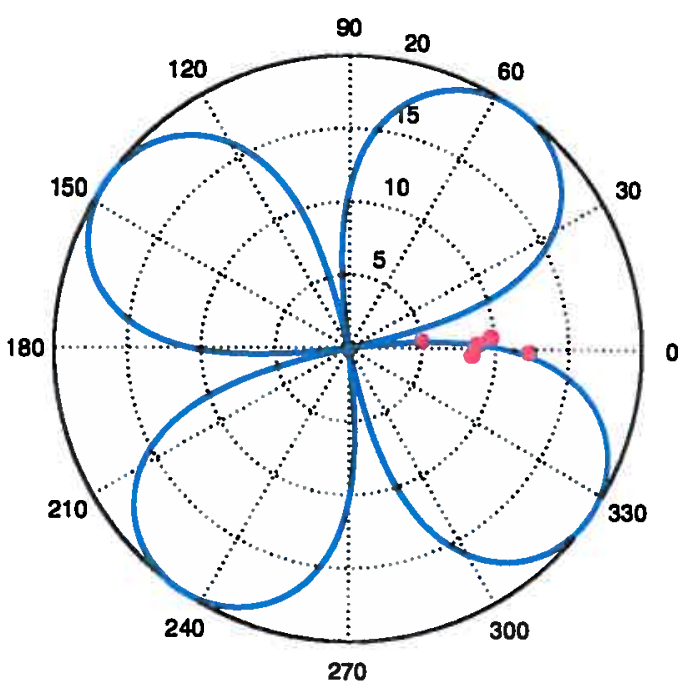

a)

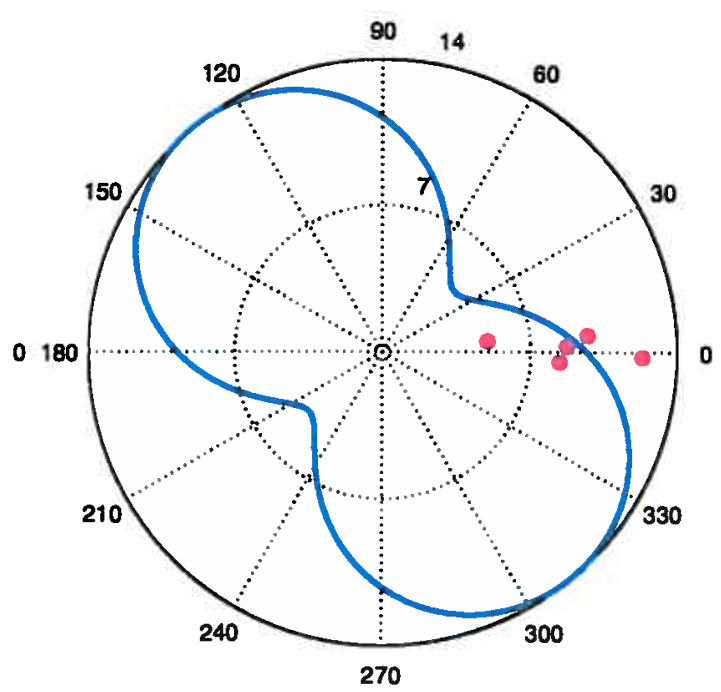

b)

Figure 5-26: Results of fits by two models (quadrupole and MD-type) for vertical component of motion during event \#16 (approximately $168 \mathrm{sec}$ from the beginning of tape RLAM-26): (a) quadrupole model, $\varphi_{0}=-10^{\circ}, \sigma_{\mathcal{M}}=4.1 \%$; (b) MD-type model, $\varphi_{0}=-40^{\circ}, \sigma_{\mathcal{M}}=11.0 \%$. Red circle markers correspond to experimental data from the five geophones. 
MD-type). The results of fits for these models are shown on Fig. 5-24. A monopole gives an even worse performance than an MD-type radiation pattern (the deviation from the monopole is two times larger than that from the MD-type pattern). Such a good discrimination between different models was possible in this case because the event was located in the immediate vicinity of the receiving array (see Fig. 5-22).

Event \#16 (approximately 168 sec from the beginning of tape RLAM-26) represents just the opposite case. It is located quite far (about $400 \mathrm{~m}$ ) from the geophone array (see Fig. 5-22), and the experimental data have a small angular spread. As a result, we could not really discriminate between the different models and therefore could not assign a corresponding event mechanism (compare Fig. 5-25 and Fig. 5-26). This case shows one of the reasons why sometimes even a qualitative determination of the event mechanism cannot be made for a successfully located event.

\subsubsection{Events at the beginning of file \#5 of tape RLAM-26}

The last group of events for which the event mechanism determination has been carried out was the group of events at the beginning of file \#5 on tape RLAM-26. These events were located using the MPD method described elsewhere in this thesis. The locations of the events discussed below are shown on Fig. 5-27.

The best-fit radiation pattern models for event \#5 (approximately 165 sec from the beginning of file \#5 on tape RLAM-26) are shown Fig. 5-28 (vertical component of motion) and Fig. 5-29 (radial and transverse components of motion). For all components the best fit is obtained by a quadrupole model which implies a strikeslip fault with a dip angle of $90^{\circ}$. This conclusion is also supported by the value of the difference in the best-fit parameter $\varphi_{0}$ between vertical and transverse components, $38^{\circ}$, and between radial and transverse components $-39^{\circ}$. In both cases this difference is quite close to $45^{\circ}$ as it should be according to numerical simulations (see Table 5.1).

The difference between $\varphi_{0}$ for the radial and transverse components is even closer to $45^{\circ}$ for event \#11 (approximately $374 \mathrm{sec}$ from the beginning of file \#5 on tape RLAM-26). For both components the best fit is achieved by a quadrupole 


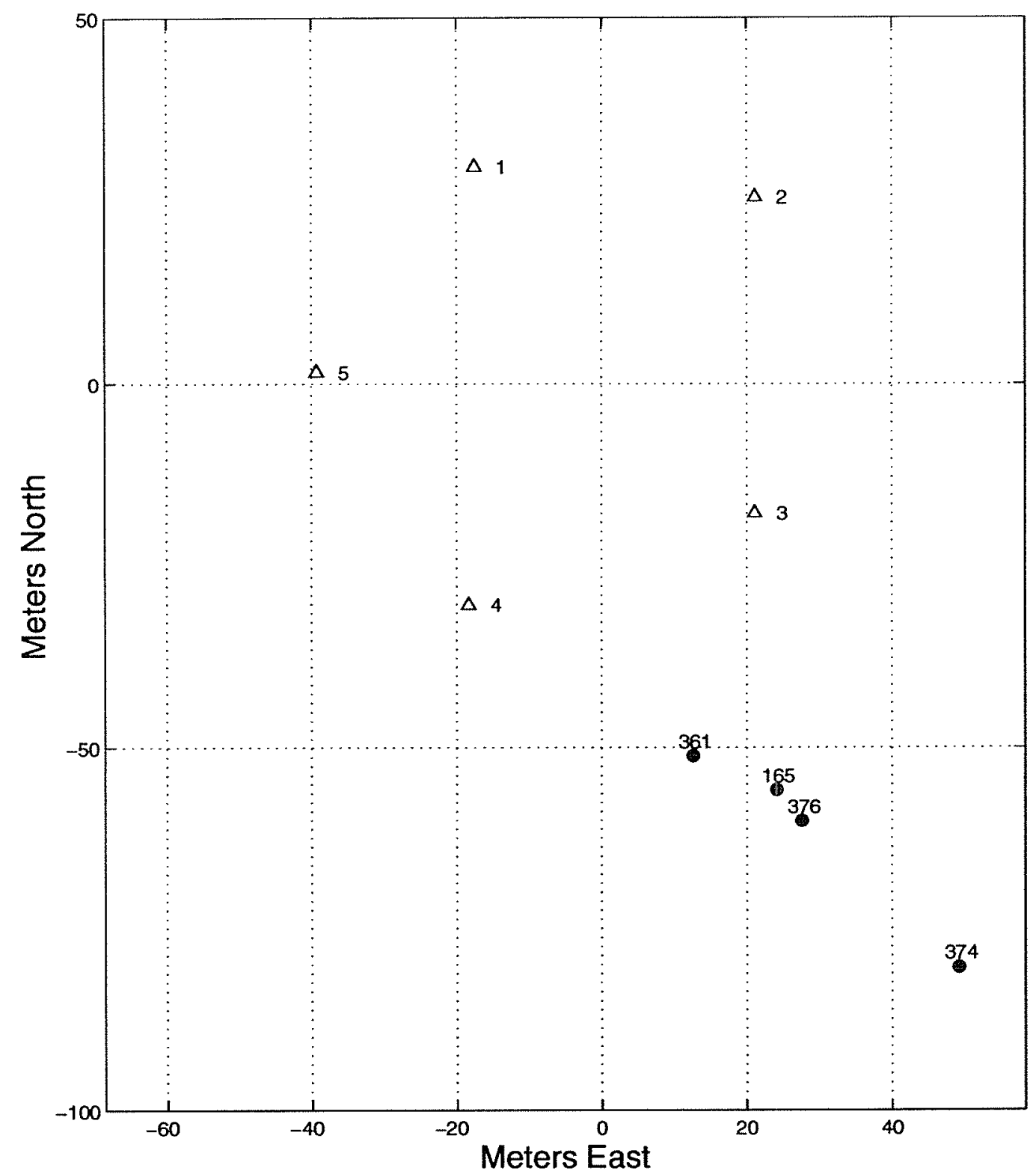

Figure 5-27: Locations of four events from file \#5 of tape RLAM-26. Triangle markers are used to show the locations of geophones, while filled circles show the locations of events. The numbers near the circle markers show approximate arrival times in seconds since the beginning of file \#5 of tape RLAM-26 for corresponding events. 


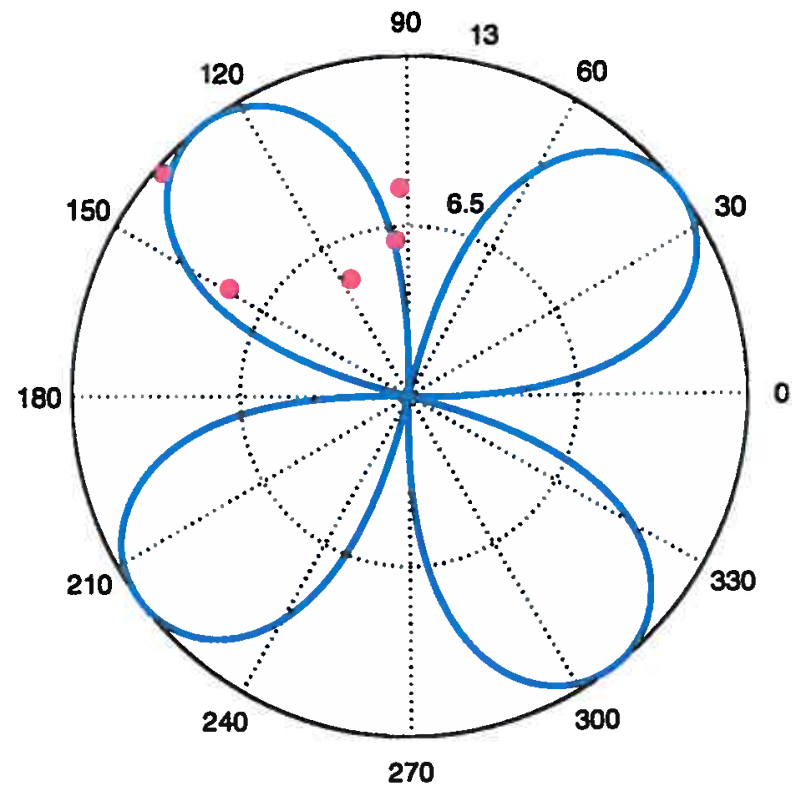

Figure 5-28: Best-fit model of radiation pattern for the vertical component of motion during event \#5 (approximately $165 \mathrm{sec}$ from the beginning of file \#5 on tape RLAM26). $\varphi_{0}=8^{\circ}, \sigma_{\mathcal{M}}=8.2 \%$. Red circle markers correspond to experimental data from the five geophones.

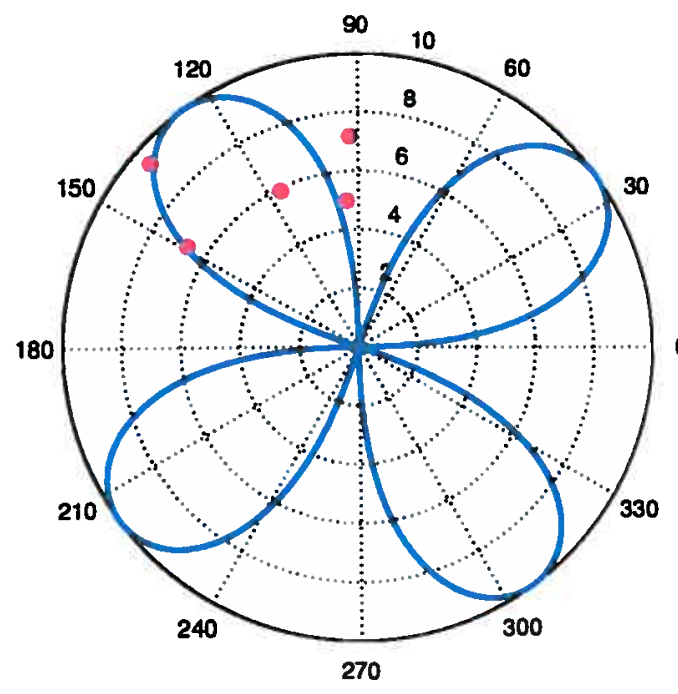

a)

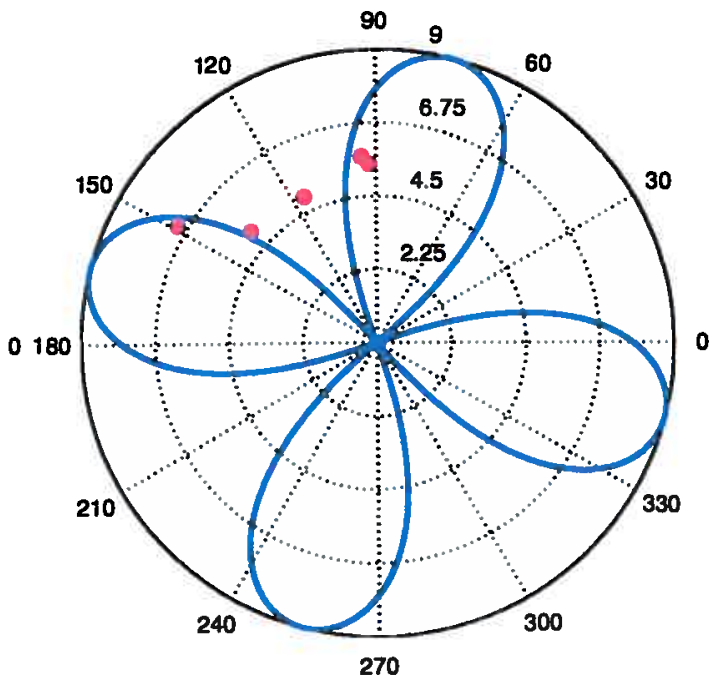

b)

Figure 5-29: Best-fit models of radiation patterns for event \#5 (approximately $165 \mathrm{sec}$ from the beginning of file \#5 on tape RLAM-26): (a) radial component of motion (to source), $\varphi_{0}=9^{\circ}, \sigma_{\mathcal{M}}=10.2 \%$; (b) transverse component of motion, $\varphi_{0}=-30^{\circ}, \sigma_{\mathcal{M}}=$ $9.8 \%$. Red circle markers correspond to experimental data from the five geophones. 


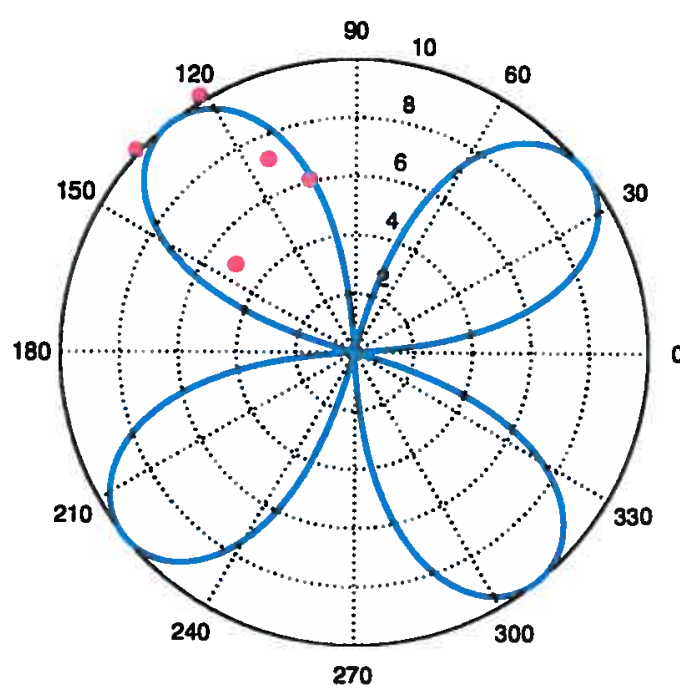

a)

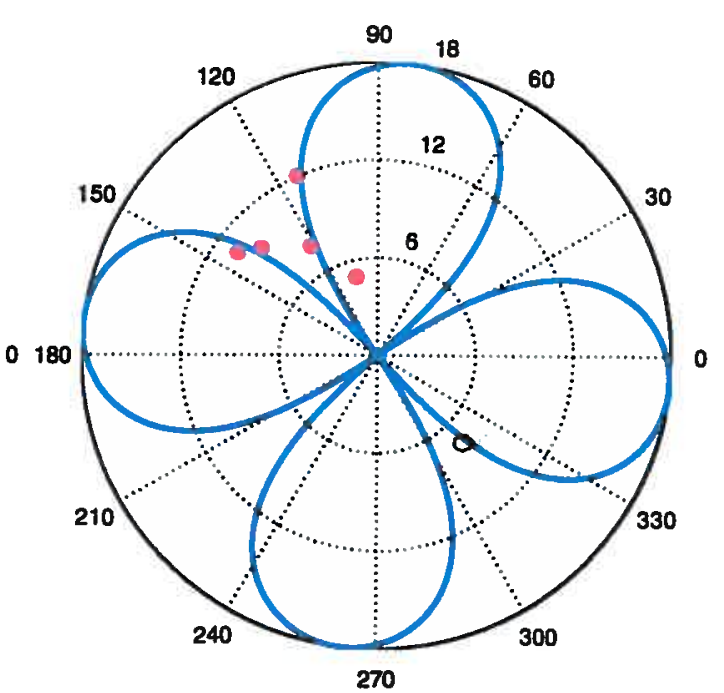

b)

Figure 5-30: Best-fit models of radiation patterns for event \#11 (approximately $374 \mathrm{sec}$ from the beginning of file \#5 on tape RLAM-26): a) radial component of motion (to source), $\varphi_{0}=6^{\circ}, \sigma_{\mathcal{M}}=6.9 \%$; b) transverse component of motion, $\varphi_{0}=-38^{\circ}, \sigma_{\mathcal{M}}=6.3 \%$. Red circle markers correspond to experimental data from the five geophones.

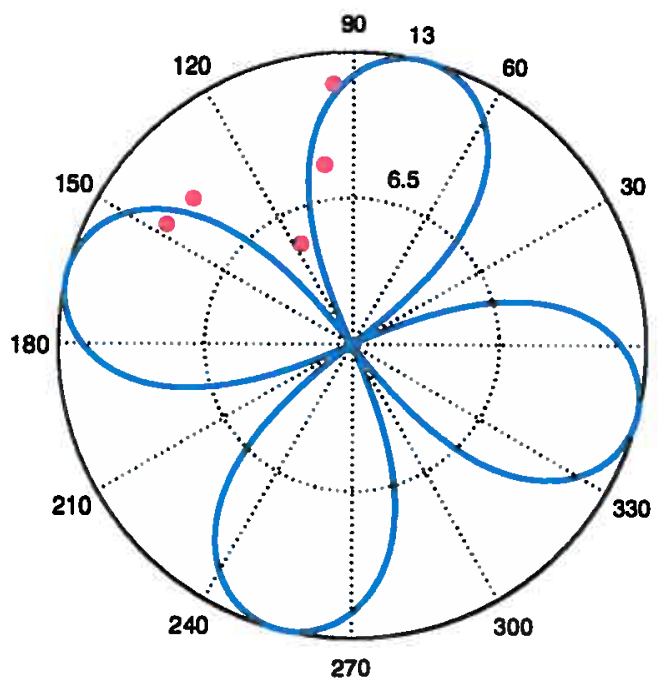

a)

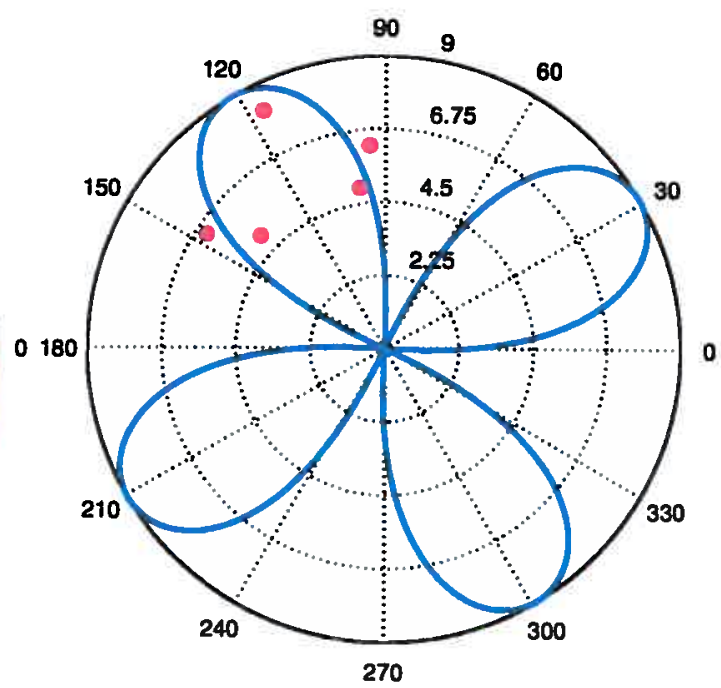

b)

Figure 5-31: Best-fit models of radiation patterns for event \#12 (approximately 376 sec from the beginning of file \#5 on tape RLAM-26): a) vertical component of motion, $\varphi_{0}=-31^{\circ}, \sigma_{\mathcal{M}}=5.5 \%$; b) transverse component of motion, $\varphi_{0}=14^{\circ}$, $\sigma_{\mathcal{M}}=7.2 \%$. Red circle markers correspond to experimental data from the five geophones. 


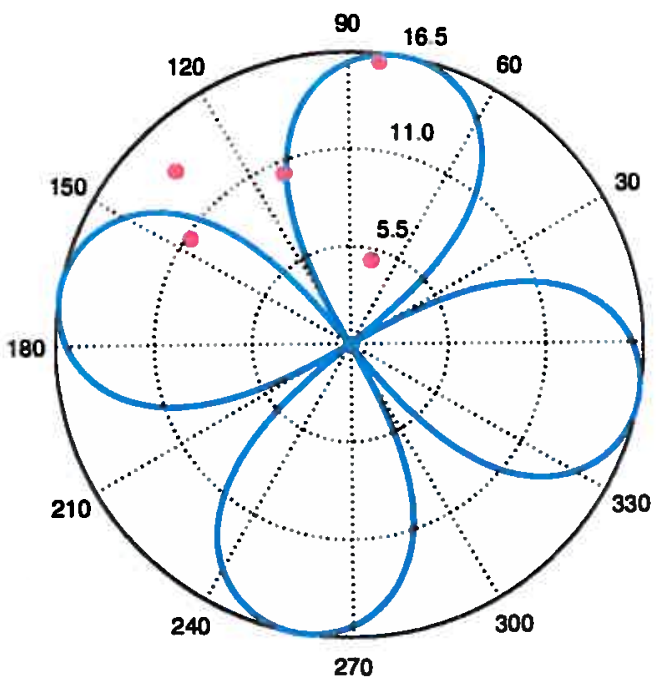

a)

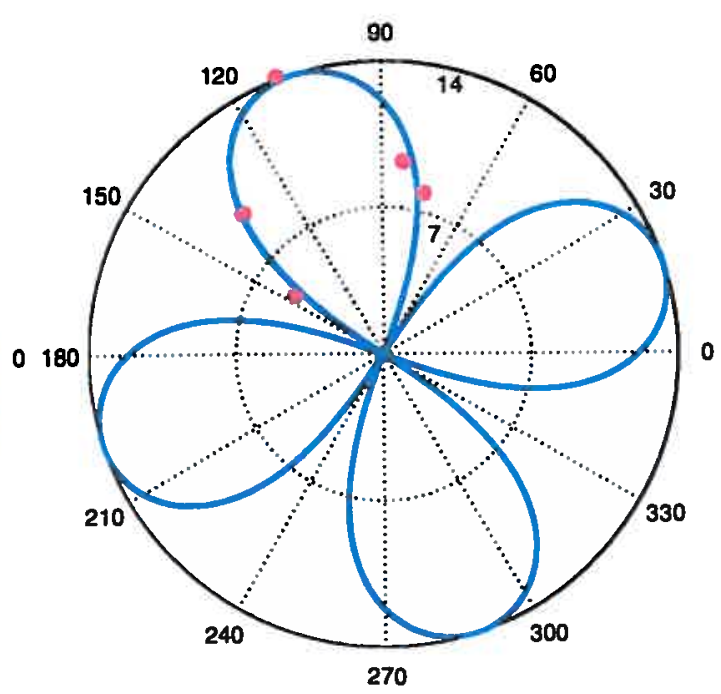

b)

Figure 5-32: Best-fit models of radiation patterns for event \#9 (approximately 361 sec from the beginning of file \#5 on tape RLAM-26): a) radial component of motion (to source), $\varphi_{0}=-34^{\circ}, \sigma_{\mathcal{M}}=10.9 \%$; (b) transverse component of motion, $\varphi_{0}=$ $27^{\circ}, \sigma_{\mathcal{M}}=2.3 \%$. Red circle markers correspond to experimental data from the five geophones.

radiation pattern (see Fig. 5-30) which corresponds to a strike-slip fault with a dip angle of $90^{\circ}$. The same event mechanism determination was made for event \#12 (see Fig. 5-31). In that case the difference in $\varphi_{0}$ was exactly $45^{\circ}$ which gives additional assurance as to a correct event mechanism identification.

For event \#9 (approximately $361 \mathrm{sec}$ from the beginning of file \#5 on tape RLAM-26) the difference in $\varphi_{0}$ between the radial and transverse components, $61^{\circ}$ (see Fig. 5-32), was not as close to $45^{\circ}$ as for events \#5, \#11 and \#12, but still within reasonable bounds $\left(61^{\circ}\right.$ is almost two times closer to $45^{\circ}$ than to $\left.90^{\circ}\right)$. The event mechanism in this case is identified as a strike-slip fault with a dip angle of $90^{\circ}$. For this event the standard deviation of the quadrupole model of radiation pattern as a function of two parameters, strike angle $\varphi_{0}$ and $-L_{\max }$, is shown on Fig. 5-33. Different colors of contours on this figure correspond to different levels of $\sigma$ with blue being the lowest level and red the highest. The minimum (or best fit) is achieved for $\varphi_{0}=27^{\circ}$ and $L_{\max }=14 \mathrm{~dB}$.

The results of the determination of fault type for several ice events are shown 


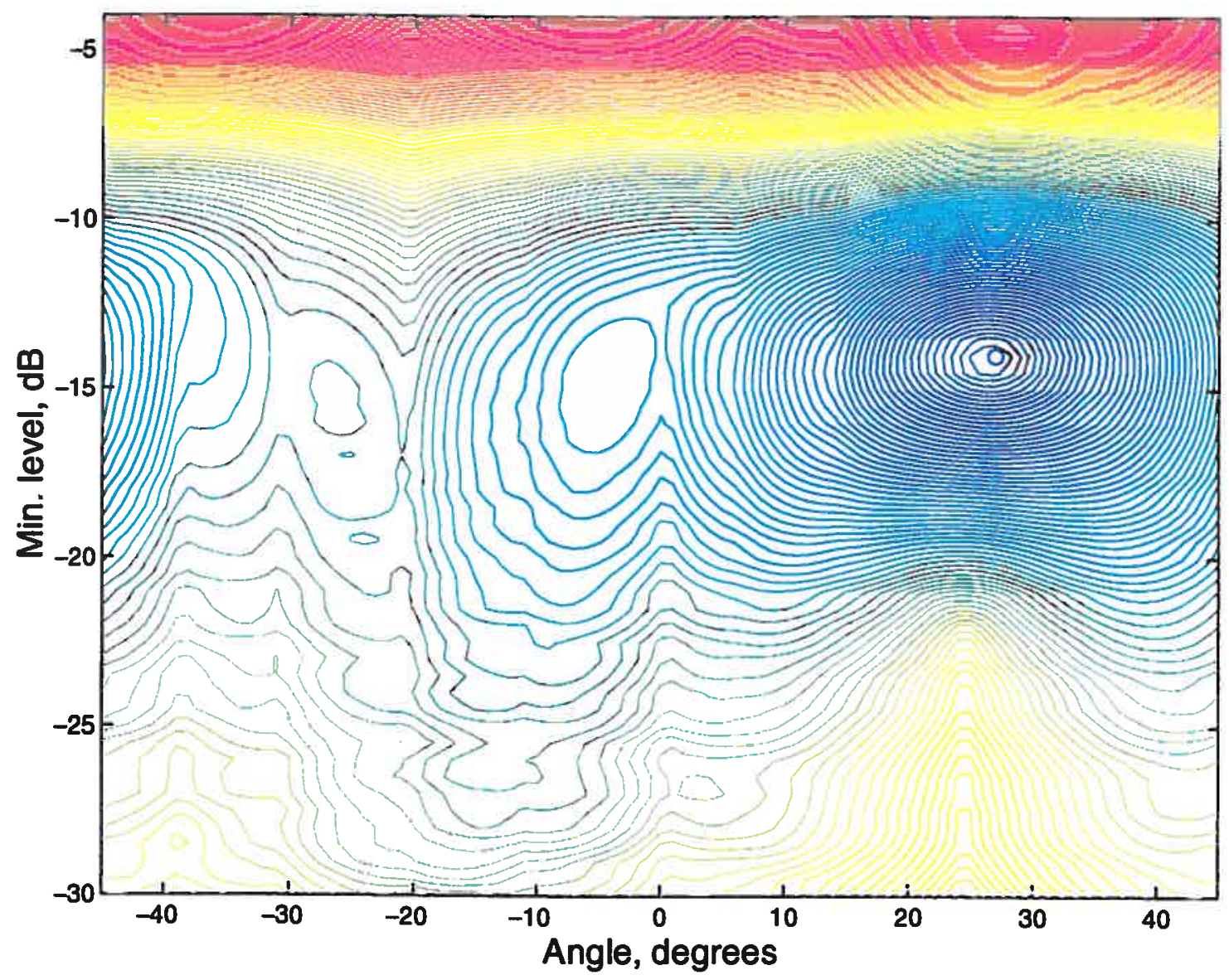

Figure 5-33: Standard deviation between experimental data and the quadrupole nodes as a function of strike angle $\varphi_{0}$ and $-L_{\max }$ (designated as "Min. level" on plot), for transverse component of motion during event \#9 (approximately $361 \mathrm{sec}$ from the beginning of file \#5 on tape RLAM-26). Different colors of contours correspond to different levels of $\sigma$ with blue being the lowest level and red the highest. The minimum (or best fit) is achieved for $\varphi_{0}=27^{\circ}$ and $L_{\max }=14 \mathrm{~dB}$. 


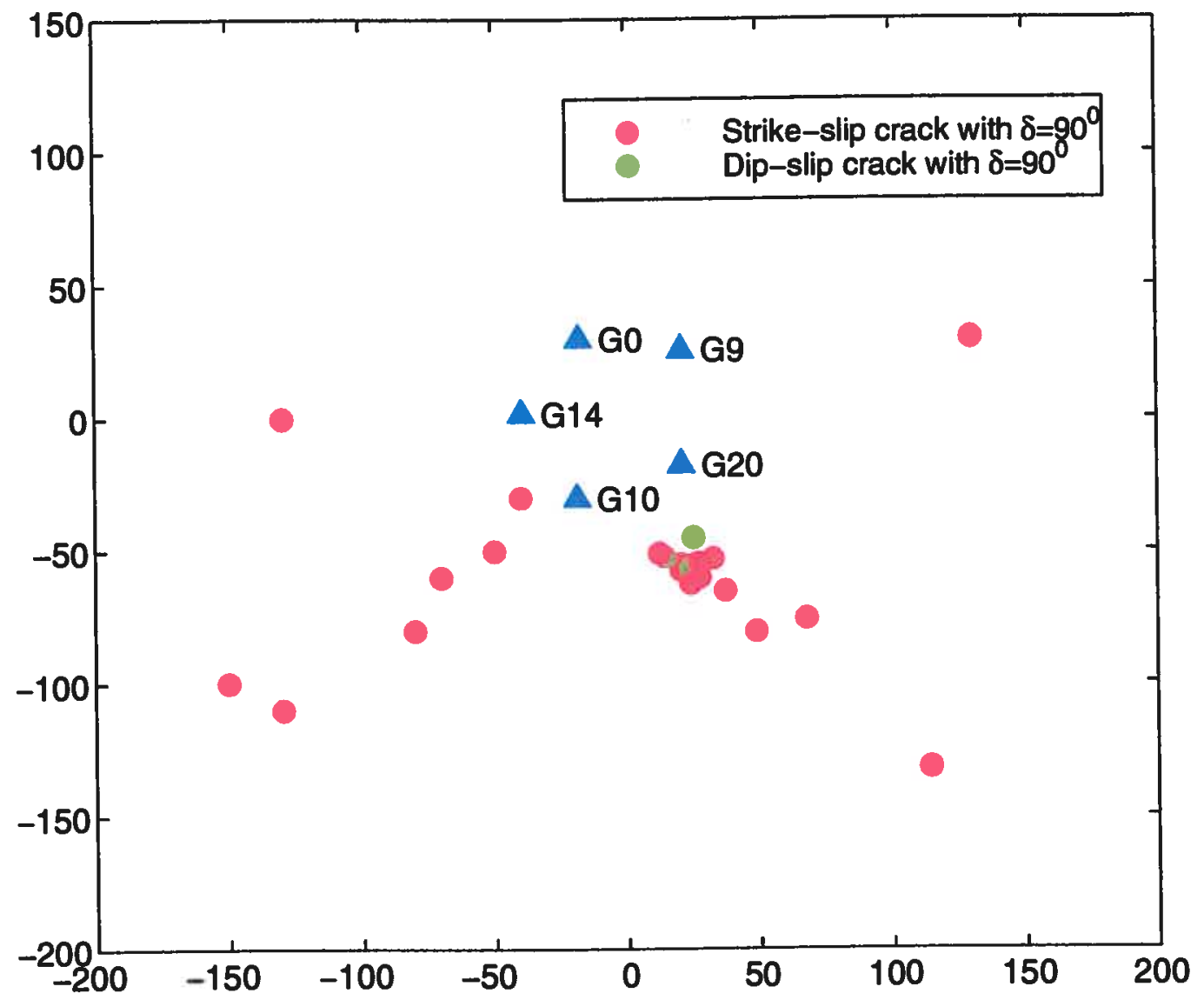

Figure 5-34: Results of the determination of fault type for several ice events from "ice island" site. 


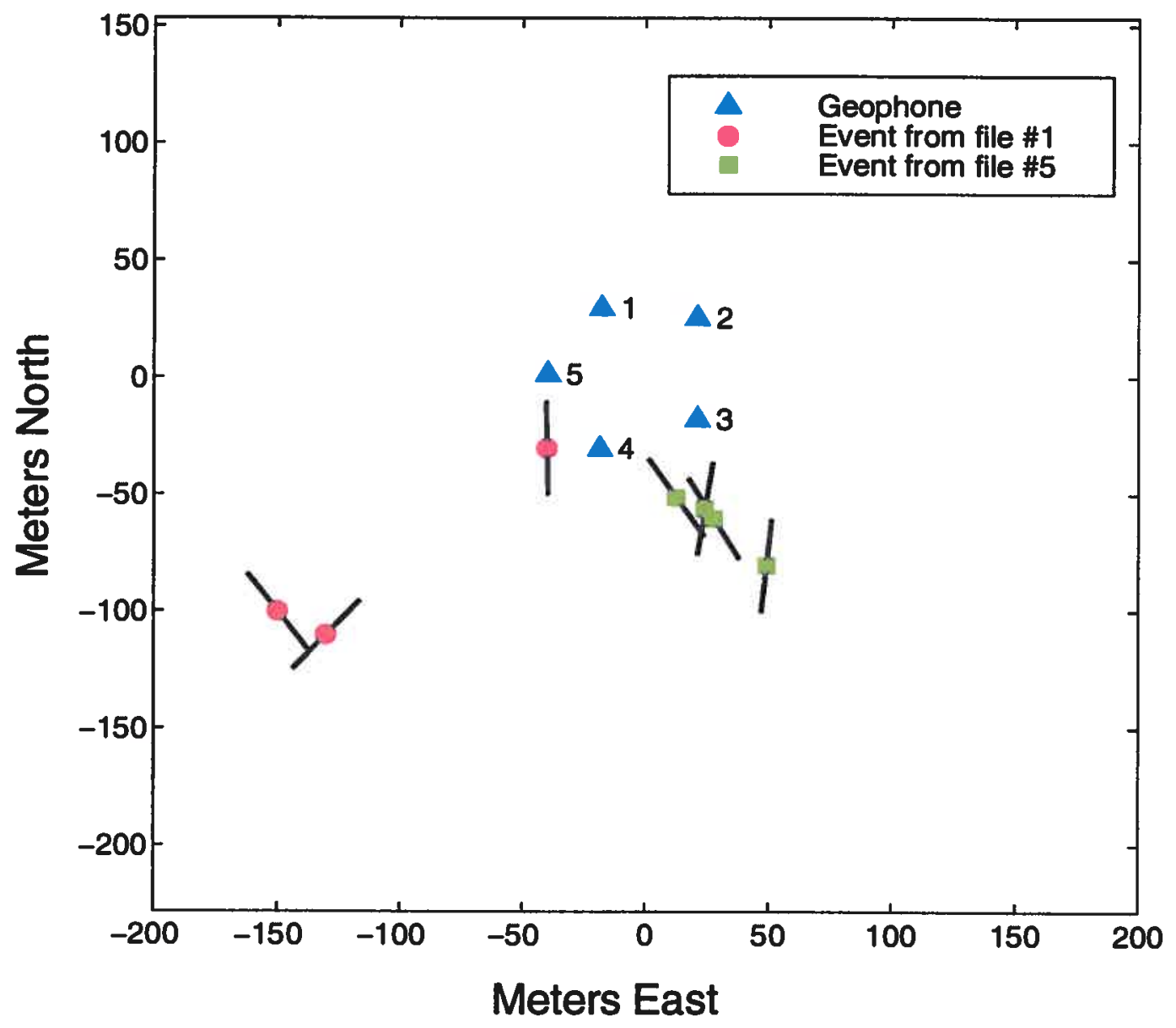

Figure 5-35: Directions of the fault motion for several events from "ice island" site. 
on Fig. 5-34. These results indicate that strike-slip faults with dip angles of $90^{\circ}$ are the most probable source mechanism. Fig. 5-35 depicts direction of the fault motion for ice events discussed in this Chapter.

\subsection{Conclusions}

In this chapter ice event identification was successfully carried out for several groups of events by comparing the quality of fit of different analytical radiation patterns to experimental data. The results of the event identification suggest that strike-slip faults with dip angles of $90^{\circ}$ are the most probable source mechanism. Additional evidence in favor of this conclusion is supplied by the differences in orientation of the best-fit radiation patterns for orthogonal components of motion which are very close to the values given by numerical models.

This choice of strike-slip fault with a dip angle of $90^{\circ}$ as the most probable source mechanism for processed ice events is somewhat to the contrary of J.S. Kim's conclusion [22] which was based on the relative smallness of the radiation from longitudinal waves compared to the acoustic mode. He suggested that dip-slip cracks with dip angles of $\delta \approx 0^{\circ}$ and $90^{\circ}$ and strike-slip cracks with a dip angle of $\delta \approx 0^{\circ}$ are the most probable source mechanisms. I think this discrepancy may arise from the fact that most of the events processed here do not radiate efficiently into the water. 


\section{Chapter 6}

\section{Event parameter estimation}

\subsection{Overview}

The similarity between physical processes in earthquakes and ice cracking has already been noted in the introduction. Based on that similarity we will use established methods from earthquake seismology to estimate the two most important parameters of faulting processes: stress drop and seismic moment. These methods can be divided into two broad classes: spectral and slip history. The spectral method of parameters estimation will be discussed in the first section along with some examples of data processing. In the next section the determination of slip history will be described and the data processing results (including stick-slip motion) will be discussed. The chapter will continue with the definition of stress drop and seismic moment for ice events. Next the procedure for the determination of event parameters from the combined results of spectral and slip history methods will be explained. Finally, the results of the event parameter estimation will be discussed and a comparison with previous estimates will be presented. 


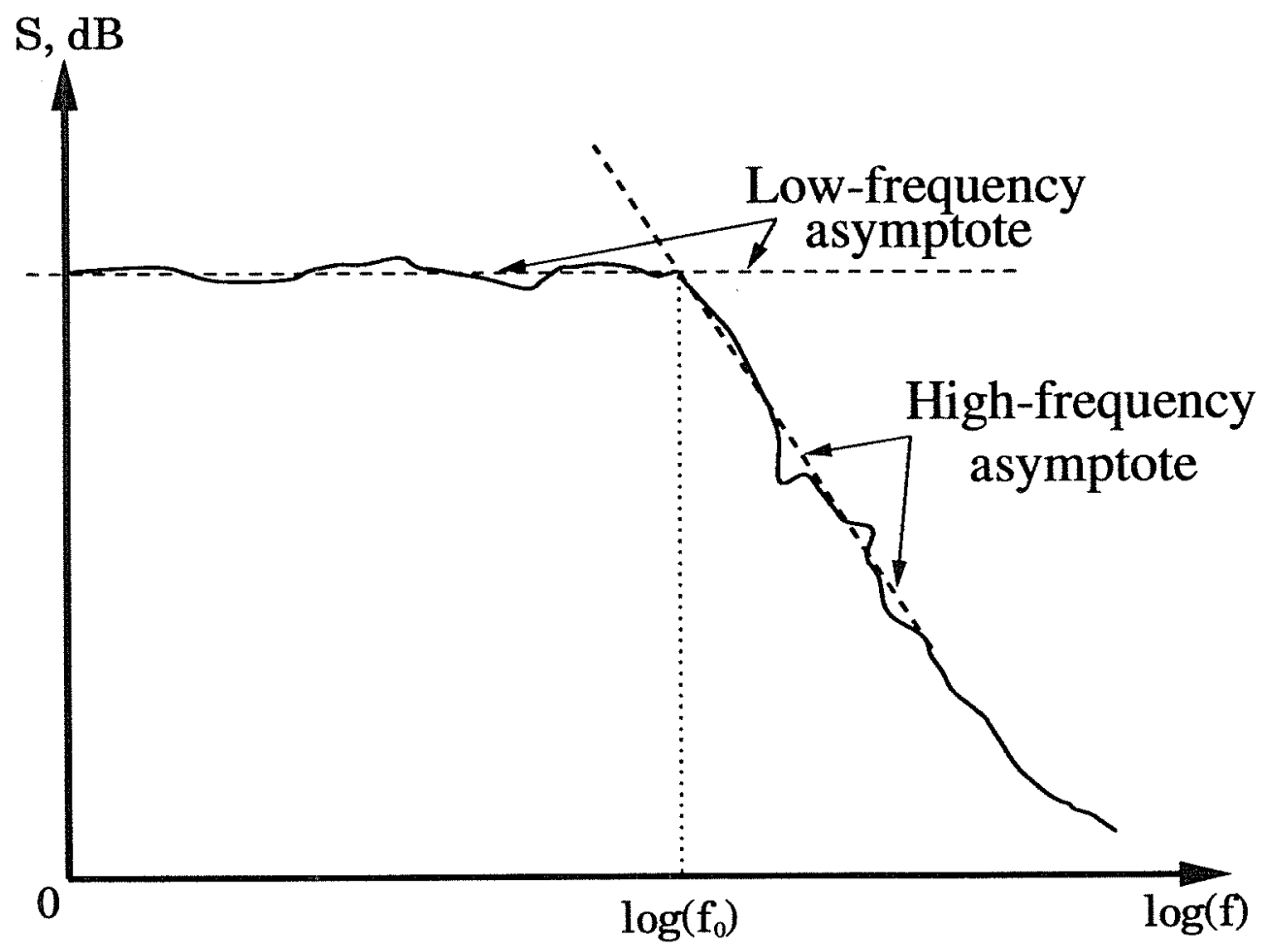

Figure 6-1: Standard model of the displacement spectrum of an earthquake. On this figure $S$ (the displacement spectrum level in $\mathrm{dB}$ ) is plotted versus the logarithm of frequency. Two asymptotes are usually associated with this spectrum: a highfrequency asymptote and a low-frequency asymptote. Their intersection defines the corner frequency $f_{0}$.

\subsection{Frequency domain processing for event param- eter estimation}

\subsubsection{Standard earthquake model of the displacement spec- trum}

In seismology the following model of displacement spectrum for a plane fault is used [48],[28]:

1. In the high frequency limit the displacement spectrum approaches an asymptote of the form $\omega^{-\gamma}$ (usually, in seismology $\gamma$ is taken to be 2).

2. The intersection of this high-frequency asymptote with the low frequency level gives the corner frequency $f_{0}$ (see Fig. 6-1) which, according to [3], is inversely 


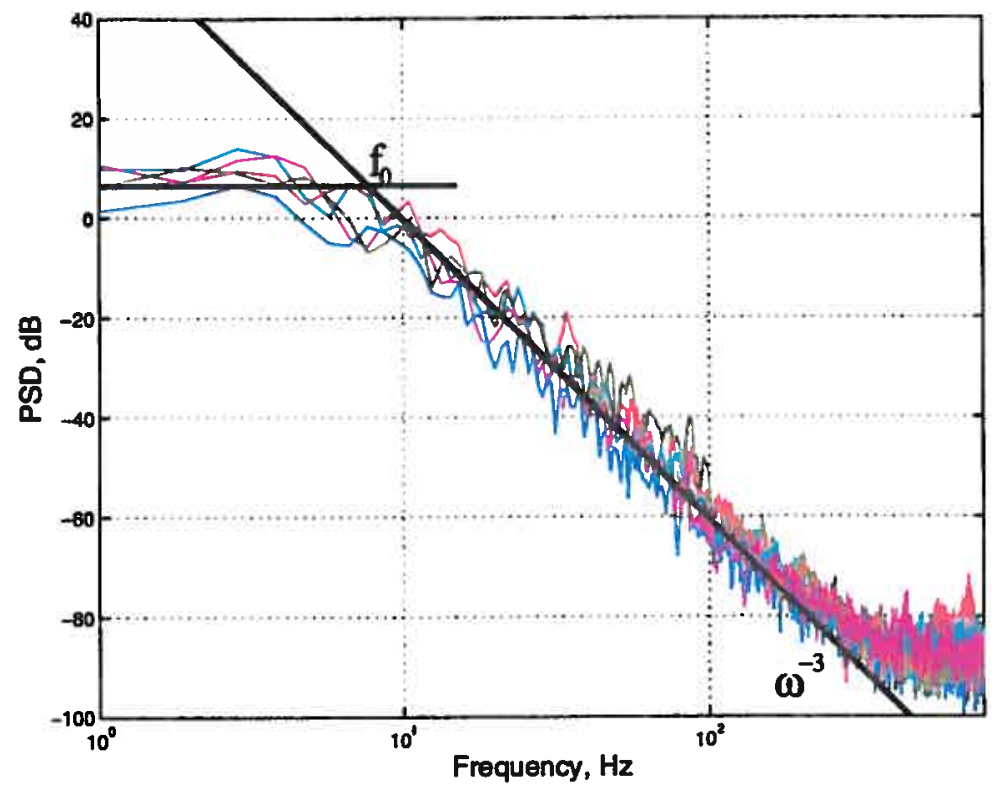

Figure 6-2: Vertical displacement spectra of the event \#3 (approximately $36.5 \mathrm{sec}$ from the beginning of the tape RLAM-26). Different colors correspond to different geophones. The high-frequency roll-off has a $\omega^{-3}$ trend (one of the black lines). The other black line shows the low-frequency trend. The intersection of the two trends gives the corner frequency $f_{0}$ as $7.8 \mathrm{~Hz}$.

related to the dimensions of the source (or source duration $T$ ): $f_{0}=1 / \pi T$.

\subsubsection{Results of corner frequency estimation}

The results of corner frequency estimation distinctly fall into two groups: the first one having only one high frequency trend $\left(\omega^{-3}\right)$ and the second one having both $\omega^{-3}$ and $\omega^{-2}$ high frequency trends.

The first group is shown on Figs. 6-2, 6-3 and 6-4. On these figures the vertical displacement spectra for several ice events are shown for all five geophones. On Fig. 62 the results of the processing of event \#3 (approximately $36.5 \mathrm{sec}$ from the beginning of tape RLAM-26) are shown. For this event the corner frequency was estimated to be $f_{0}=7.8 \mathrm{~Hz}$. For event \#5, which occured approximately $39 \mathrm{sec}$ from the beginning of tape RLAM-26, the spectral method gave the following value of corner frequency: $f_{0}=8.8 \mathrm{~Hz}$ (see Fig. 6-3). The results of the processing of event \#9 (approximately $58 \mathrm{sec}$ from the beginning of tape RLAM-26) are shown on Fig. 6-4. In this case the 


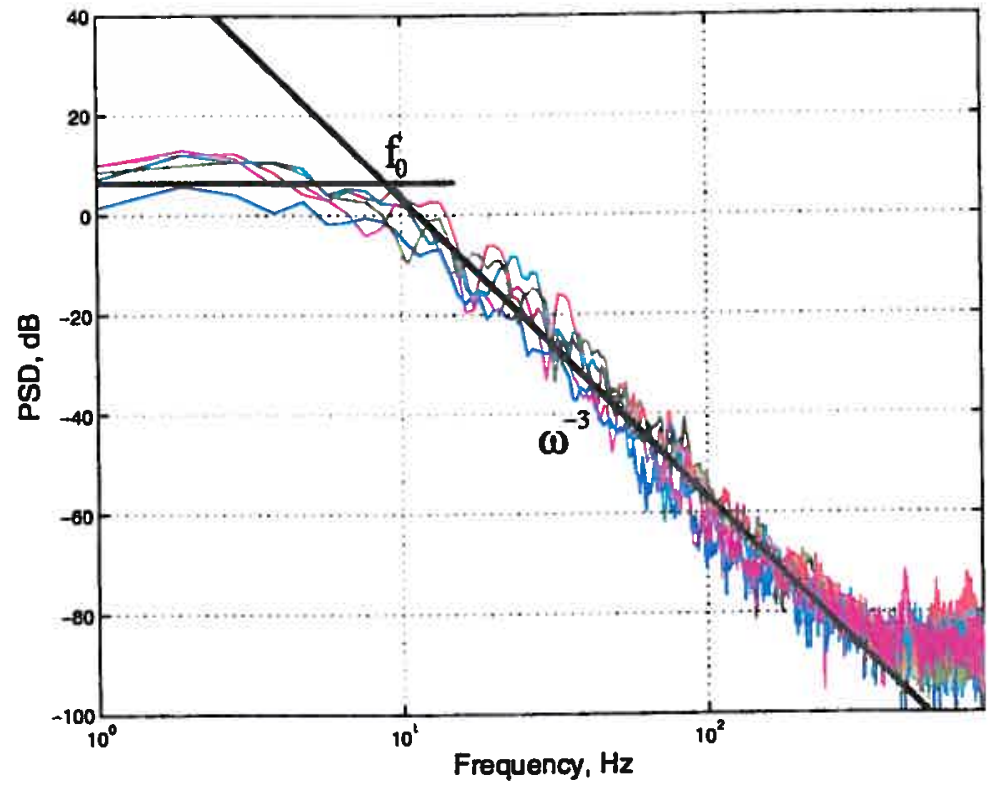

Figure 6-3: Vertical displacement spectra of event \#5 (approximately $39 \mathrm{sec}$ from the beginning of tape RLAM-26). Different colors correspond to different geophones. The high-frequency roll-off follows a $\omega^{-3}$ trend (one of the black lines). The other black line shows the low-frequency trend. Their intersection gives the corner frequency $f_{0}=8.8 \mathrm{~Hz}$.

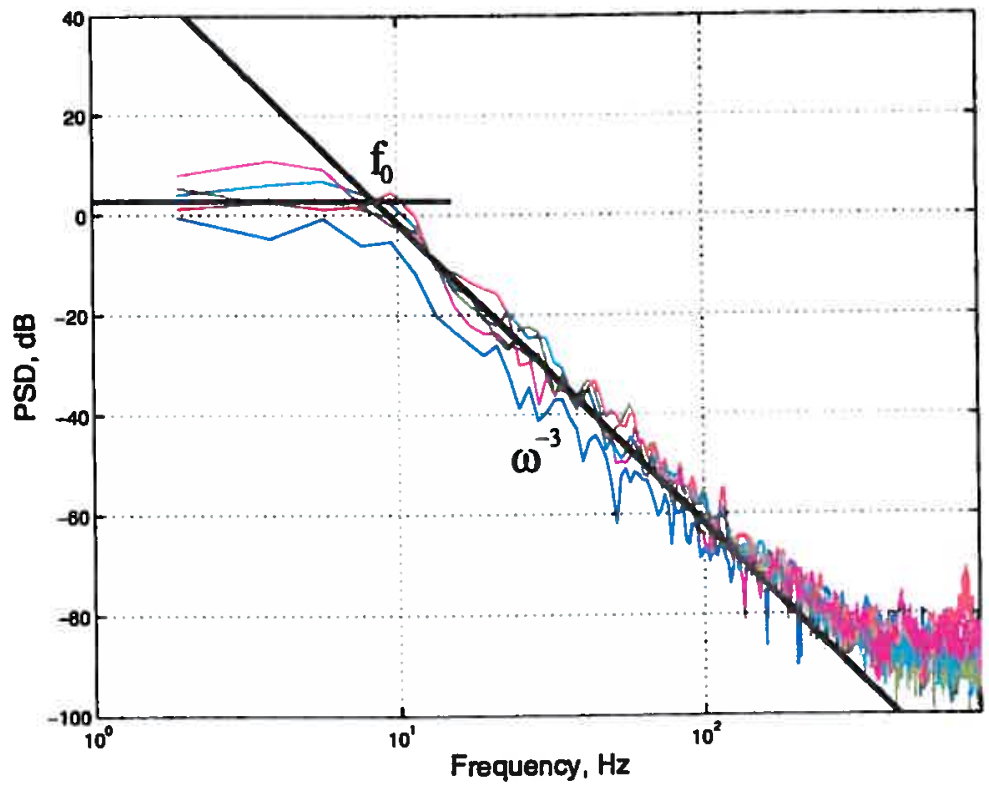

Figure 6-4: Vertical displacement spectra of event \#9 (approximately $58 \mathrm{sec}$ from the beginning of tape RLAM-26). Different colors correspond to different geophones. The high-frequency roll-off follows a $\omega^{-3}$ trend (one of the black lines). The other black line shows the low-frequency trend. Their intersection gives the corner frequency $f_{0}=8.4 \mathrm{~Hz}$. 


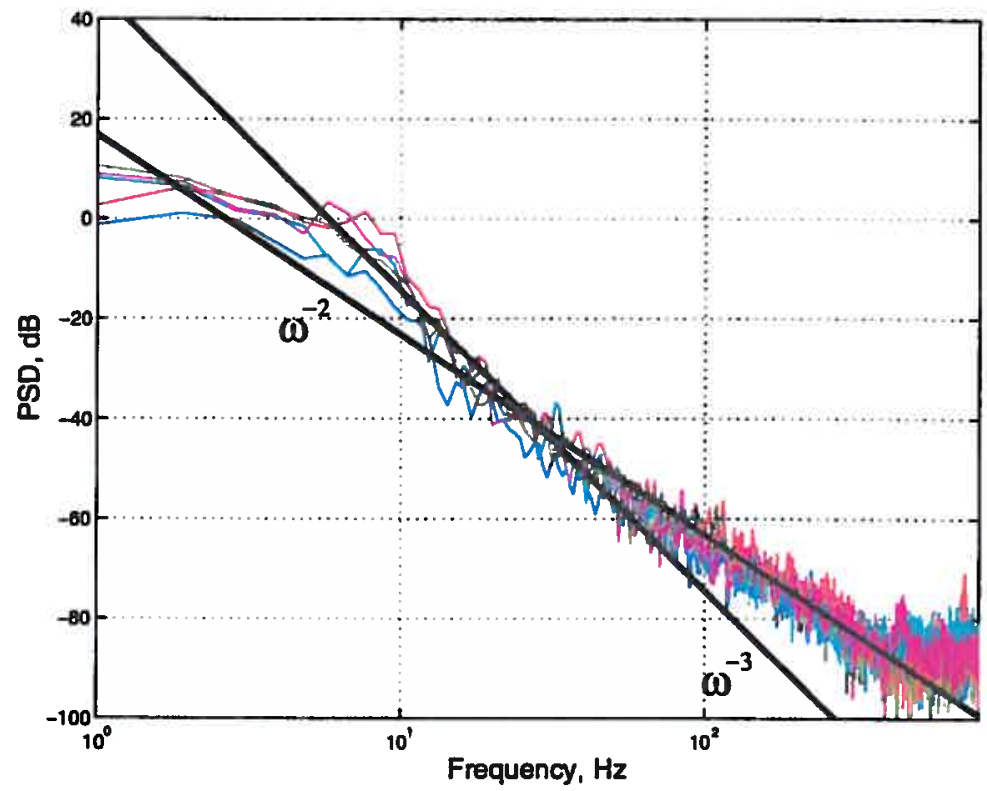

Figure 6-5: Vertical displacement spectra of the event \#11 (approximately $74 \mathrm{sec}$ from the beginning of tape RLAM-26). Different colors correspond to different geophones. For this event two high-frequency trends can be observed. First, there is a $\omega^{-3}$ trend (one of the black lines), then a $\omega^{-2}$ trend (the second black line). No corner frequency could be identified in this case.

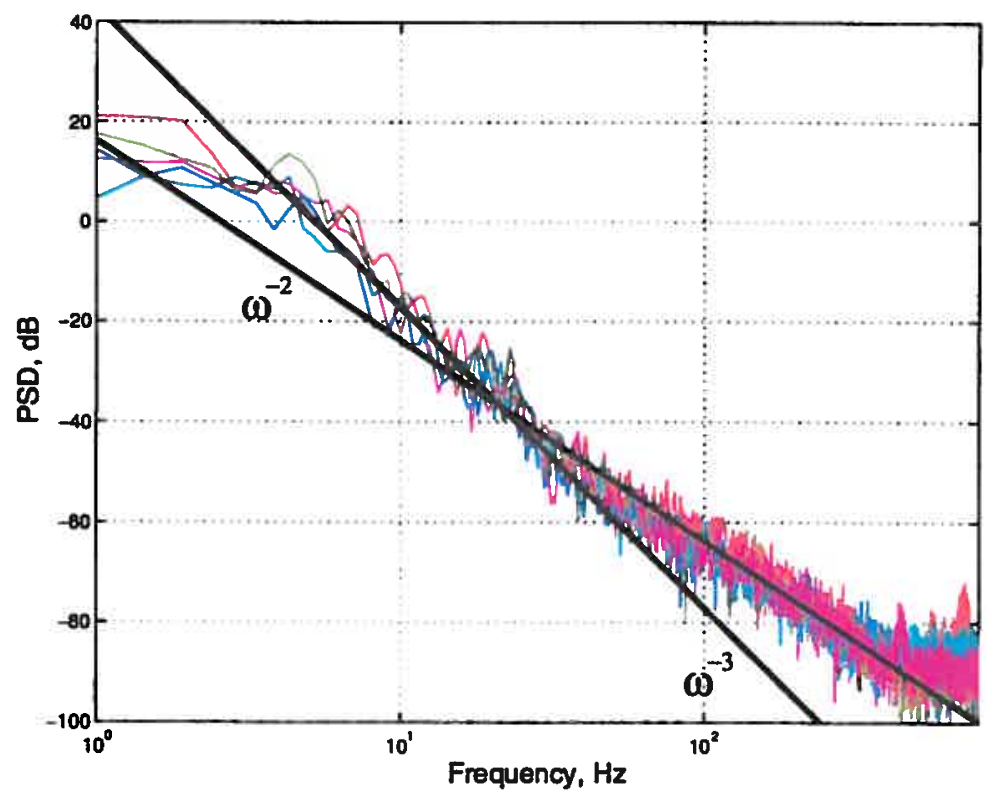

Figure 6-6: Vertical displacement spectra of the event \#12 (approximately $80 \mathrm{sec}$ from the beginning of the tape RLAM-26). Different colors correspond to different geophones. For this event two high-frequency trends can be observed. First, there is a $\omega^{-3}$ trend (one of the black lines), then a $\omega^{-2}$ trend (the second black line). No corner frequency could be identified in this case. 


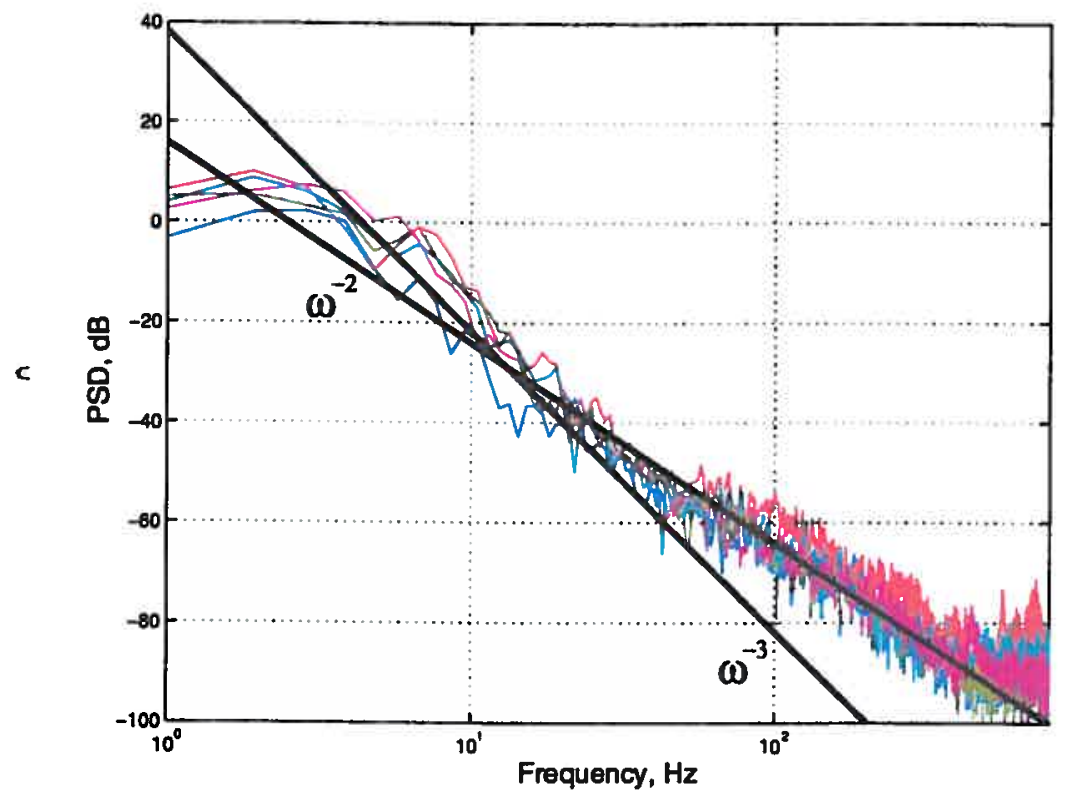

Figure 6-7: Vertical displacement spectra of event \#13 (approximately $118 \mathrm{sec}$ from the beginning of tape RLAM-26). Different colors correspond to different geophones. For this event two high-frequency trends can be observed. First, there is a $\omega^{-3}$ trend (one of the black lines), then a $\omega^{-2}$ trend (the second black line). No corner frequency could be identified in this case.

corner frequency value was estimated to be $f_{0}=8.4 \mathrm{~Hz}$.

The second group of events is shown on Figs. 6-5, 6-6 and 6-7. The results of the processing for event \#11 (approximately $74 \mathrm{sec}$ from the beginning of tape RLAM-26) are shown on Fig. 6-5, for event \#12 (approximately $80 \mathrm{sec}$ from the beginning of the tape RLAM-26) on Fig. 6-6, and for event \#13 (approximately $118 \mathrm{sec}$ from the beginning of the tape RLAM-26) on Fig. 6-7. On these figures the vertical displacement spectra for several ice events are shown for all five geophones. The common feature of the results of processing in this group is that the displacement spectra have two high-frequency trends starting with $\omega^{-3}$ trend and ending with $\omega^{-2}$ trend. Due to the ambiguity of this situation, no corner frequency could be identified in this case.

In earthquake mechanics a $\omega^{-3}$ trend is usually associated with the process of nucleation of the fault [1]. On the basis of only limited geophone coverage of the faulting processes this hypothesis could be neither confirmed nor denied for the discussed ice events, but it could be considered as a plausible explanation for this 
phenomenon.

\subsection{Slip history determination}

\subsubsection{Two models for the slip history of the fault}

The two most widely accepted theories in seismology of the slip history on the fault are models of Haskell [16] and Brune [3].

In the Haskell's model [16] (see Fig. 6-8a) the displacement on the fault $u(t)$ could be expressed as

$$
u(t)=u_{\infty} G\left(t-x / v_{r}\right)
$$

where $v_{r}$ is the rupture propagation velocity, and $G(t)$ is a ramp function. The ramp function is zero at $t<0$ and increases linearly with time until it reaches 1 at $t=T_{R}$, which is called the rise time (for asymptotic curves, the time to reach $90 \%$ of the final level of the curve is sometimes called the rise time).

In Brune's model [3] (see Fig. 6-8b) we have for the displacement

$$
u(t)=u_{\infty}\left(1-e^{-\frac{i_{0} t}{u_{\infty}}}\right) \quad \text { for } t \geq 0
$$

For the initial particle velocity this theory gives

$$
\dot{u}_{0}=\frac{\sigma}{\mu} c_{s}
$$

where:

$\sigma$ is the initial stress level,

$\mu$ is the shear strength of the medium,

$c_{s}$ (or $\beta$ as it is usually denoted in seismological literature) is the shear wave speed of the medium. 


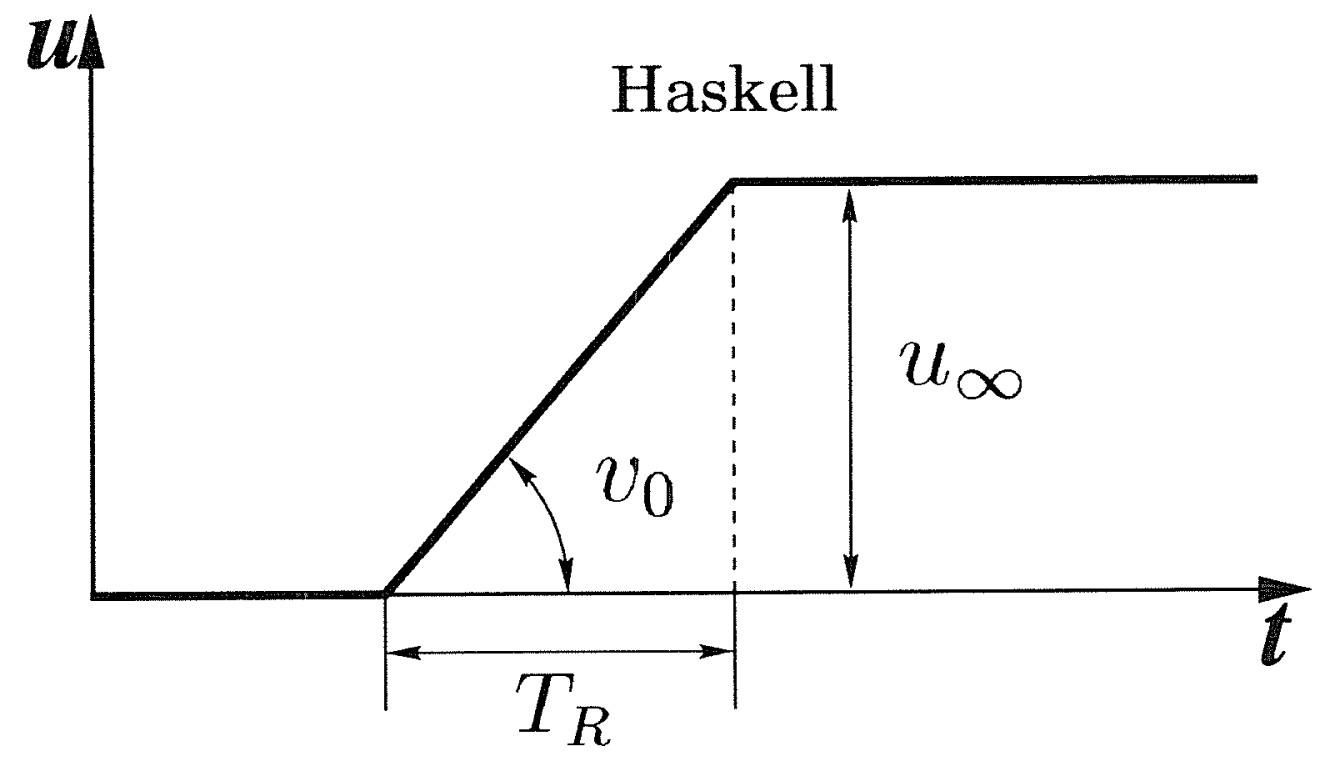

(a)

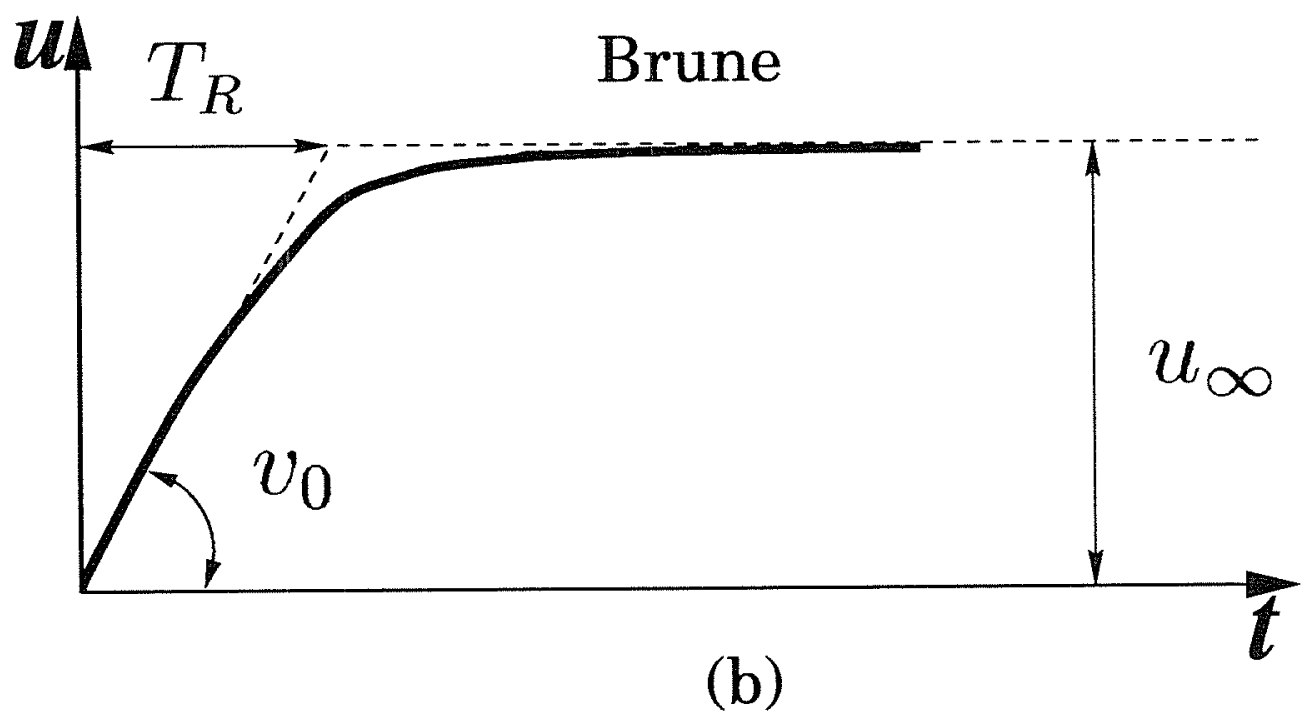

Figure 6-8: Two standard models of slip history accepted in seismology: (a) Haskell's model; (b) Brune's model. On this figure for both models the following parameters are defined: $v_{0}$ - initial velocity (in text, it is also called $\dot{u}_{0}$ ), $u_{\infty}-$ maximum slip, $T_{R}$ - rise time. 
In the seismological community Brune's model is usually preferred over Haskell's model as it more closely describes the physics of faulting. Brune's model takes into the account the finiteness of the fault dimensions, while Haskell's does not. For the following discussion, Brune's model will be used.

According to Kasahara ([21], p. 108), an average particle velocity associated with faulting may be derived simply by dividing the slip amplitude by rise time. In other words we have

$$
\dot{u}_{0}=\frac{u_{\infty}}{T_{R}},
$$

where:

$\dot{u}_{0}$ is the initial (or average) velocity, $u_{\infty}$ is the slip amplitude.

For event parameter estimation the most important parameter is the average slip $\bar{u}$ which represents a slip amplitude $u_{\infty}$ which has been averaged in some way. 


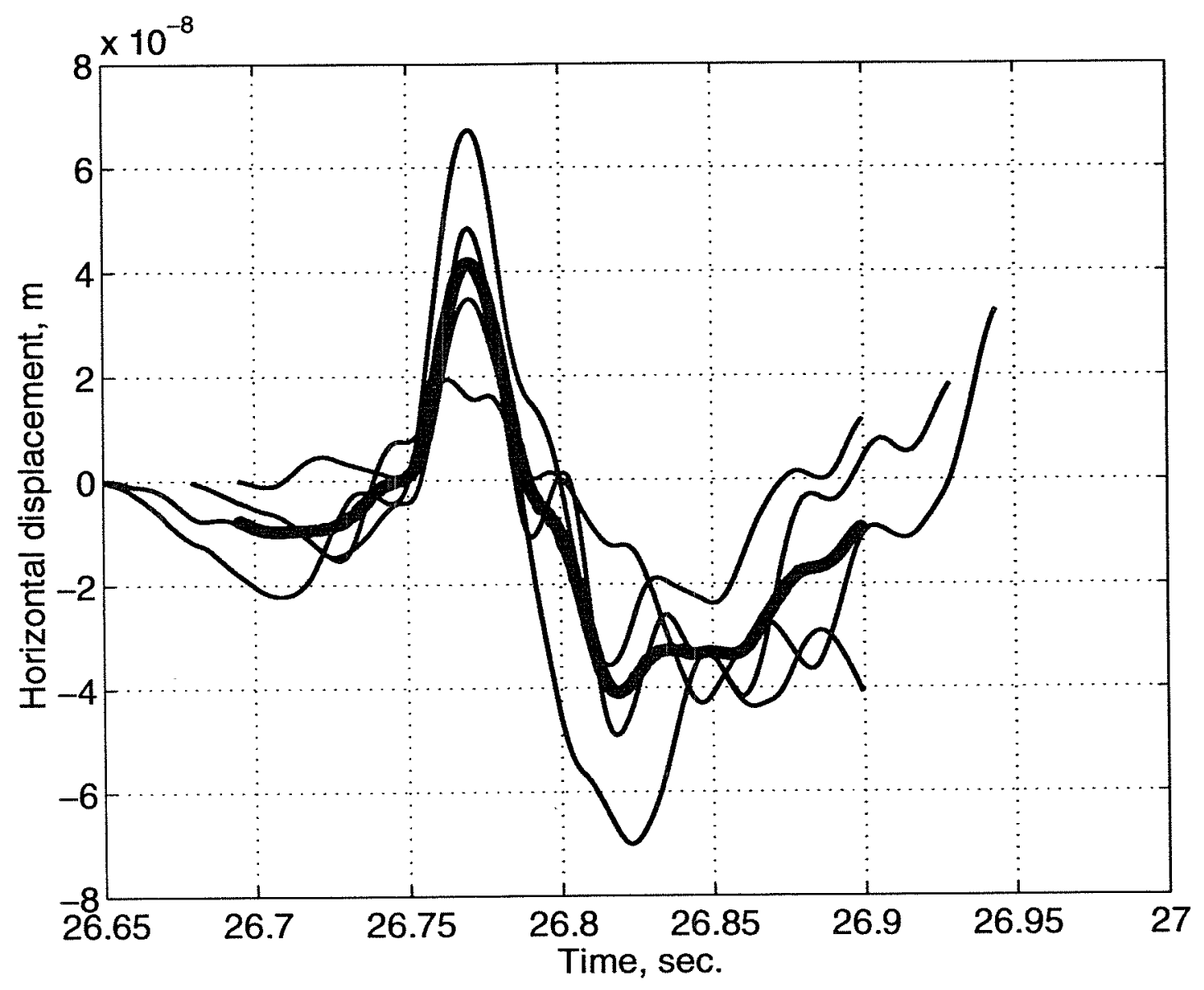

Figure 6-9: Slip history of event \#1 (approximately $27 \mathrm{sec}$ from the beginning of tape RLAM-26). Only the horizontal X-component (true North) of the displacement is shown on this plot. Four thin lines correspond to 4 geophones appropriately shifted in time in order to align their peaks. The thick line shows the average across 4 geophones aligned in time. This curve gives a rise time of approximately $62 \mathrm{msec}$ and a slip amplitude of $5.13 \times 10^{-8} \mathrm{~m}$.

\subsubsection{Results of slip history determination}

\section{Simple slip histories}

On Fig. 6-9 you can see the slip history of event \#1 (approximately 27 sec from the beginning of tape RLAM-26). Only the horizontal X-component (true North) of the displacement is shown on this plot. Note that at the end the displacement does not come to a constant level as in the model of slip history. The oscillations at the end of the event could be explained by hi-pass filtering at the geophone itself: the low frequency cutoff of each geophone was $4.5 \mathrm{~Hz}$ at which frequency the frequency 


\section{Slip history for 2 events}
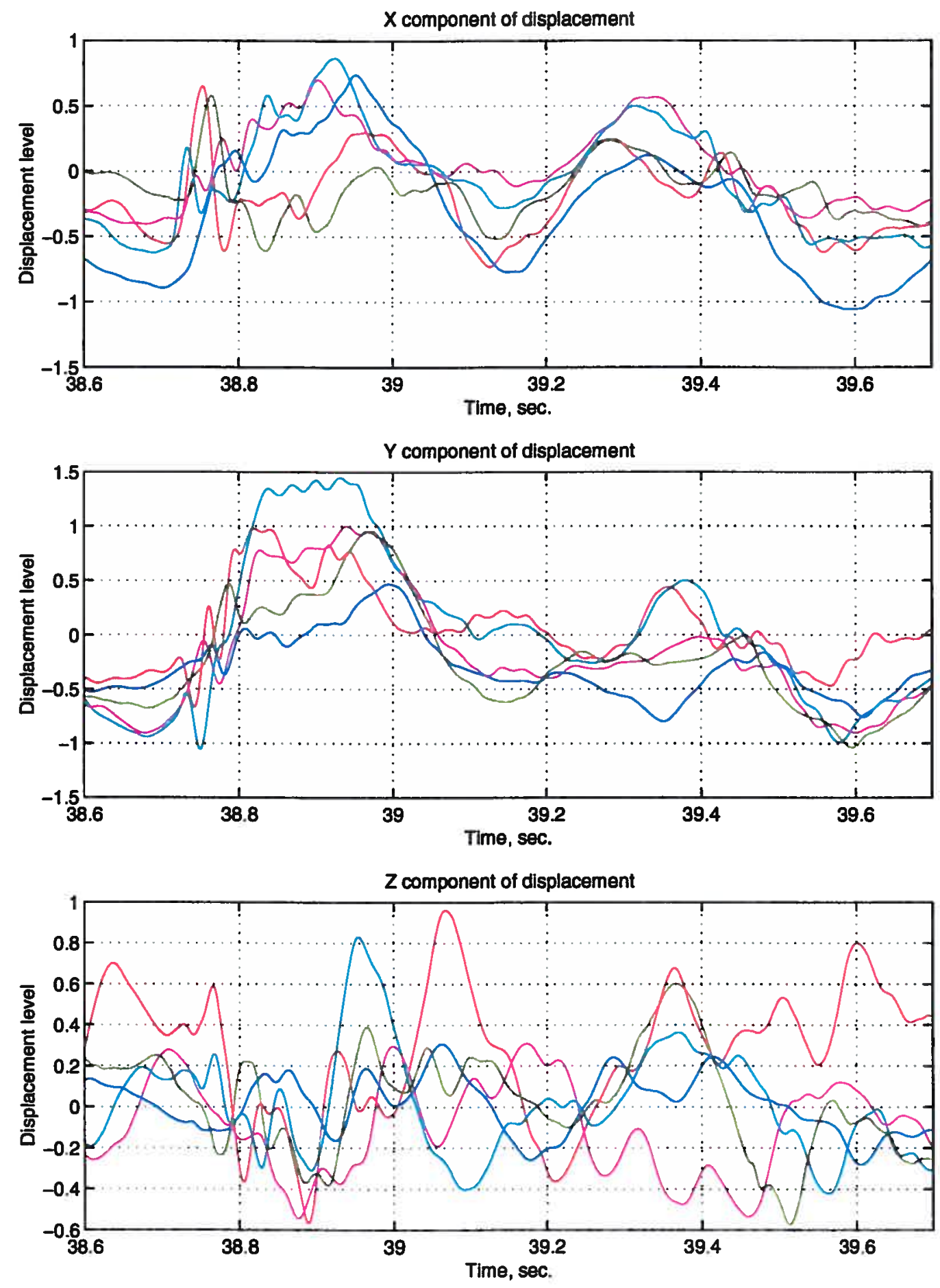

Figure 6-10: Slip history of two events at about $38 \mathrm{sec}$. from the beginning of the tape RLAM-26. All three components of displacement are shown. On this plot the units of displacement are arbitrary. The scales are shown for separate events. 

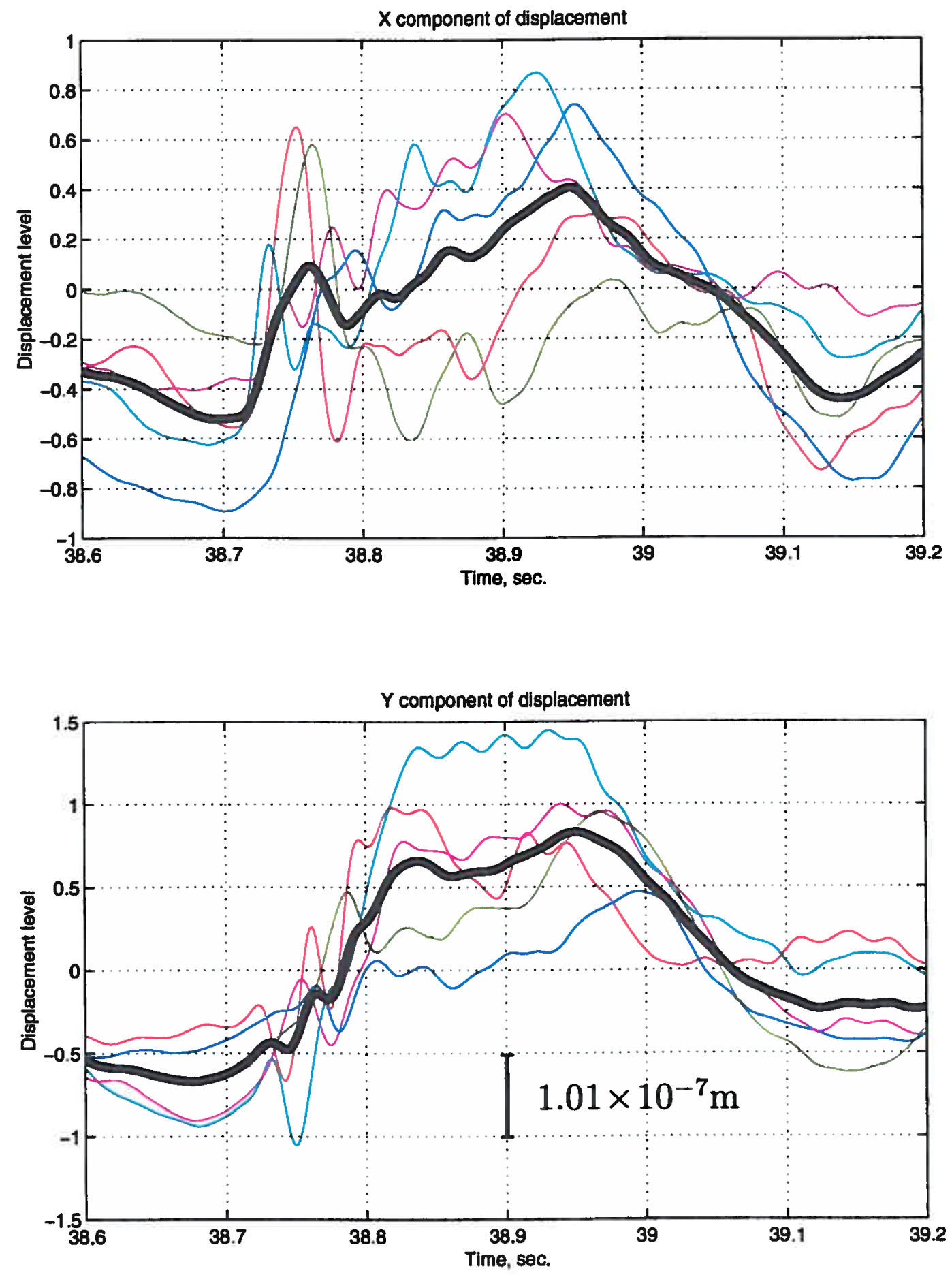

Figure 6-11: Slip history of the first event from Fig. 6-10. Only the horizontal components of displacement are shown. On this plot the units of displacement are arbitrary. The scale is shown on the plot of the Y-component. The thick line shows the average across all 5 geophones (the alignment was not really needed in this case unlike the case of Fig. 6-9). 

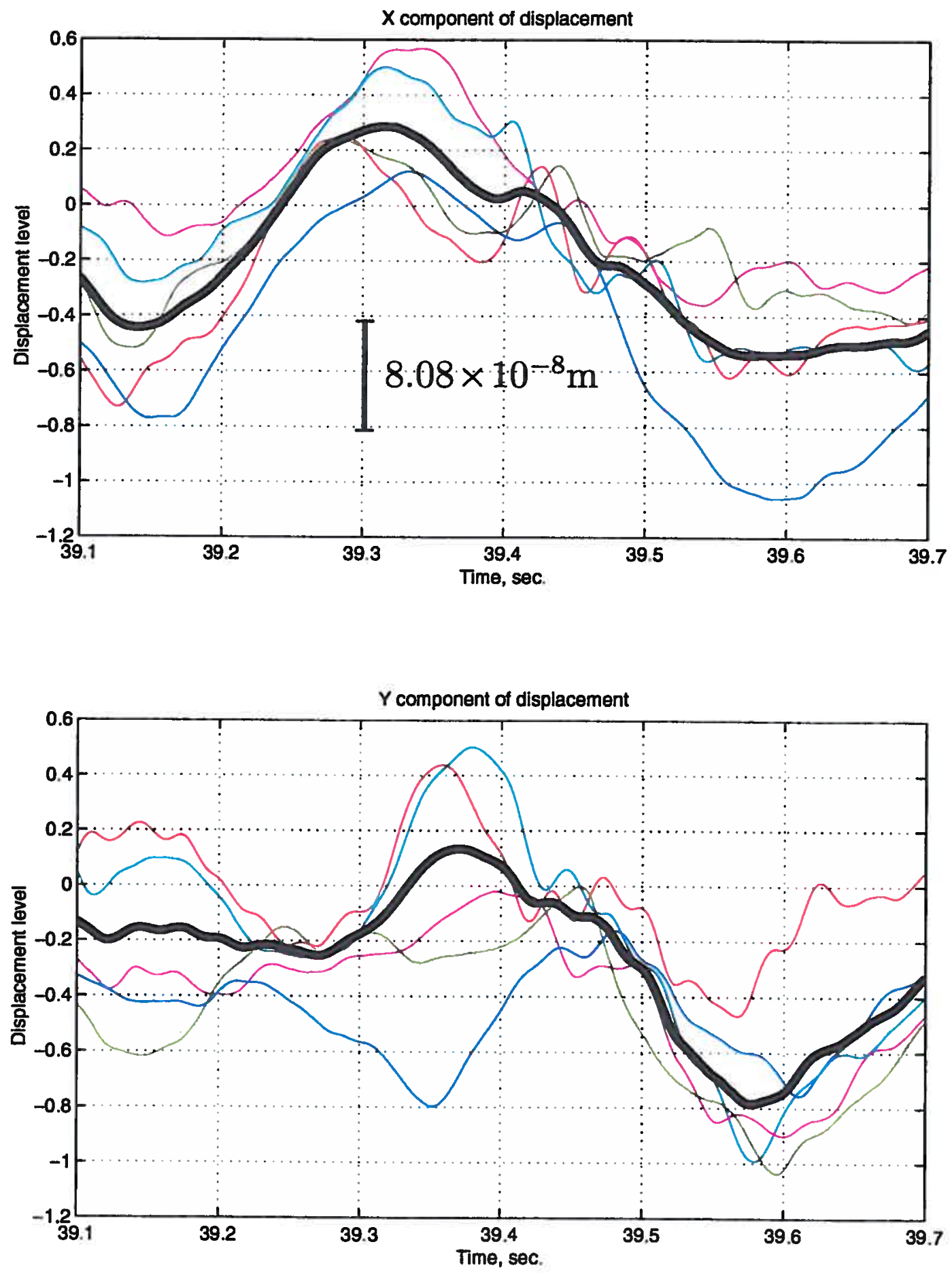

Figure 6-12: Slip history of the second event from Fig. 6-10. Only the horizontal components of displacement are shown. On this plot the units of displacement are arbitrary. The scale is shown on the plot of the X-component. The thick line shows the average across all 5 geophones (the alignment was not really needed in this case unlike in Fig. 6-9). 
response was already $3 \mathrm{~dB}$ down. Looking at the figure we could see that the frequency of the final oscillation is close to the low frequency cutoff.

The average across the geophones in this case gives a rise time of $62 \mathrm{msec}$ and a slip amplitude of $5.13 \times 10^{-8} \mathrm{~m}$. From Equation (6.4) we now obtain

$$
\dot{u}_{0}=8.28 \times 10^{-6} \mathrm{~m} / \mathrm{s} .
$$

On Fig. 6-10 the slip histories of two events (approximately $38 \mathrm{sec}$ from the beginning of tape RLAM-26) are shown for three components of motion. For the first event (see Fig. 6-11) the determination of the rise time could best be achieved from the Y-component of the displacement (true West). The rise time in this case is about $110 \mathrm{msec}$, the slip amplitude (before correction) is $2.43 \times 10^{-7} \mathrm{~m}$. According to Equation (6.4) that gives the following value of initial velocity

$$
\dot{u}_{0}=2.21 \times 10^{-6} \mathrm{~m} / \mathrm{s}
$$

For the second event (see Fig. 6-12) the determination of the rise time can best be achieved from the X-component of displacement (true North). The rise time in this case is about $120 \mathrm{msec}$ and the slip amplitude (before correction) is $1.42 \times 10^{-7} \mathrm{~m}$. Now, according to Equation (6.4) we get the following value for initial velocity:

$$
\dot{u}_{0}=1.18 \times 10^{-6} \mathrm{~m} / \mathrm{s} .
$$

Note that above observation about final oscillations applies also in these last two cases.

\section{Stick-slip history}

Brace and Byerlee showed [2] that previously faulted surfaces of rock may undergo a series of intermittent small slips under continuing pressure. They called this kind of motion "stick-slip" by analogy with the term used in materials science. Processes in stick-slip were described by Byerlee as follows 


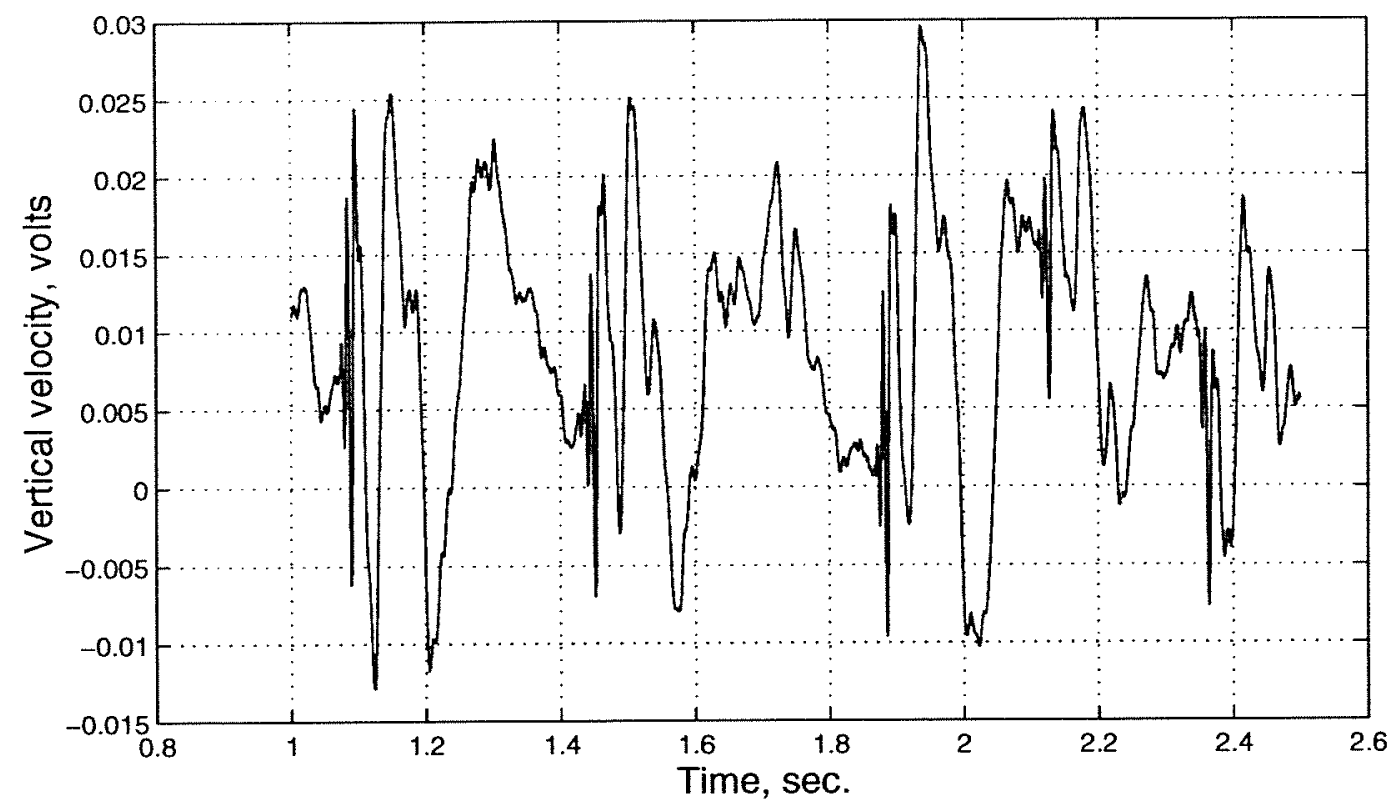

(a)

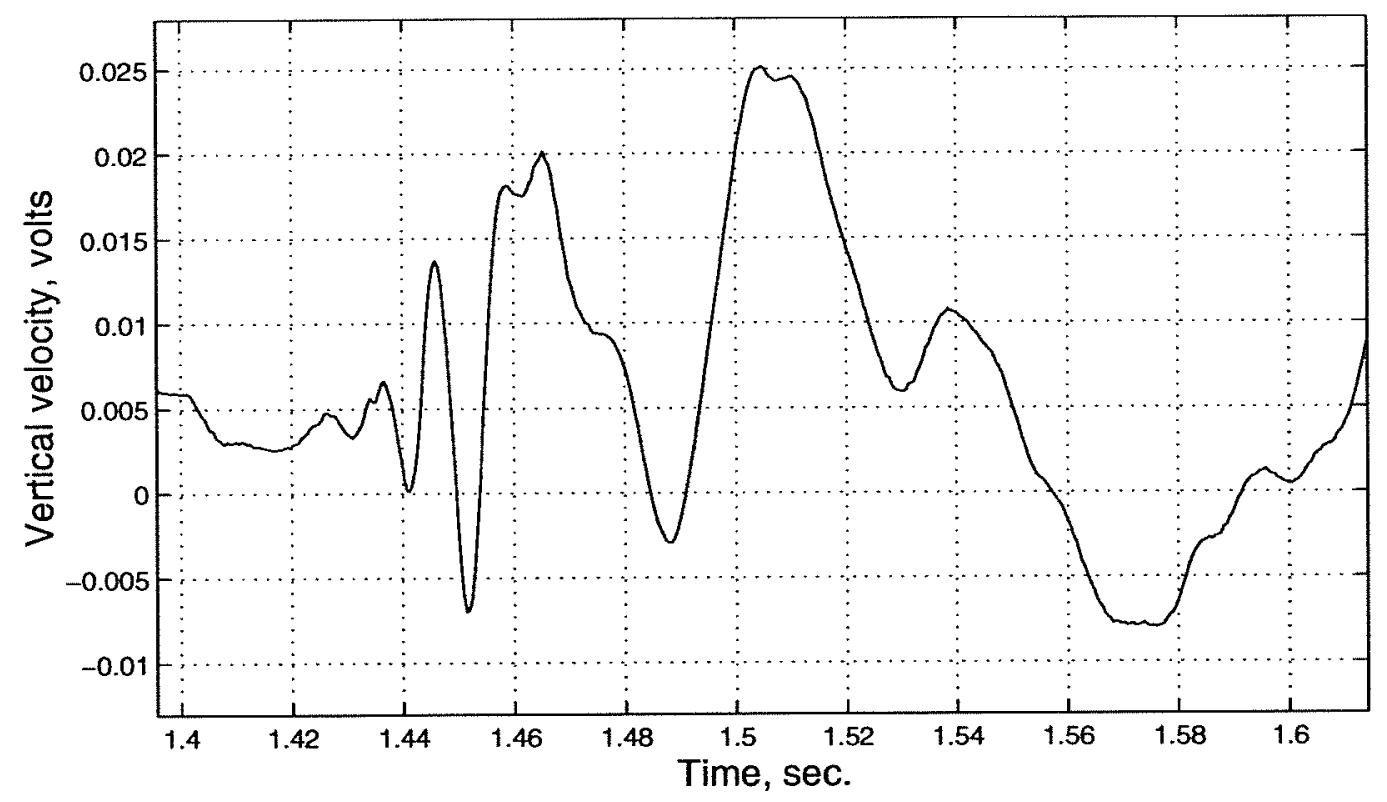

(b)

Figure 6-13: Time series of the vertical component of velocity on the geophone \#2 at the beginning of tape RLAM-26. Note several pulses similar in shape at approximately $1.1 \mathrm{sec}, 1.4 \mathrm{sec}$ and $1.8 \mathrm{sec}$ on the top plot (a). The bottom plot (b) shows the details of the pulse at $1.4 \mathrm{sec}$. The characteristic arrival pattern indicates a flexural wave. 


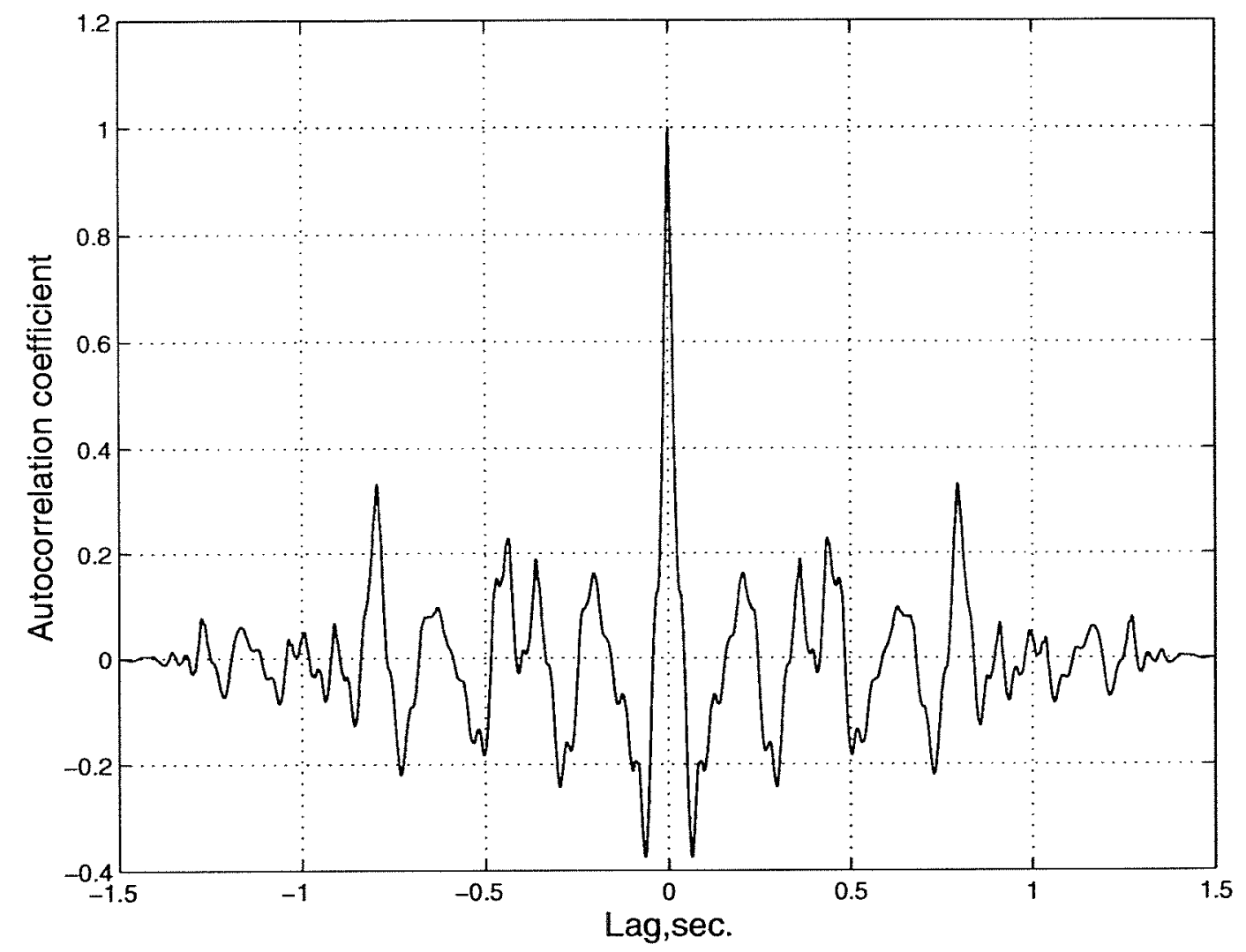

Figure 6-14: Autocorrelation function for the time series of the vertical component of velocity on geophone \#2 at the beginning of tape RLAM-26 (see Fig. 6-13). Note the significant peaks at a lag of about $0.8 \mathrm{sec}$ and some secondary peaks at about $0.35 \mathrm{sec}$ and $0.45 \mathrm{sec}$. 


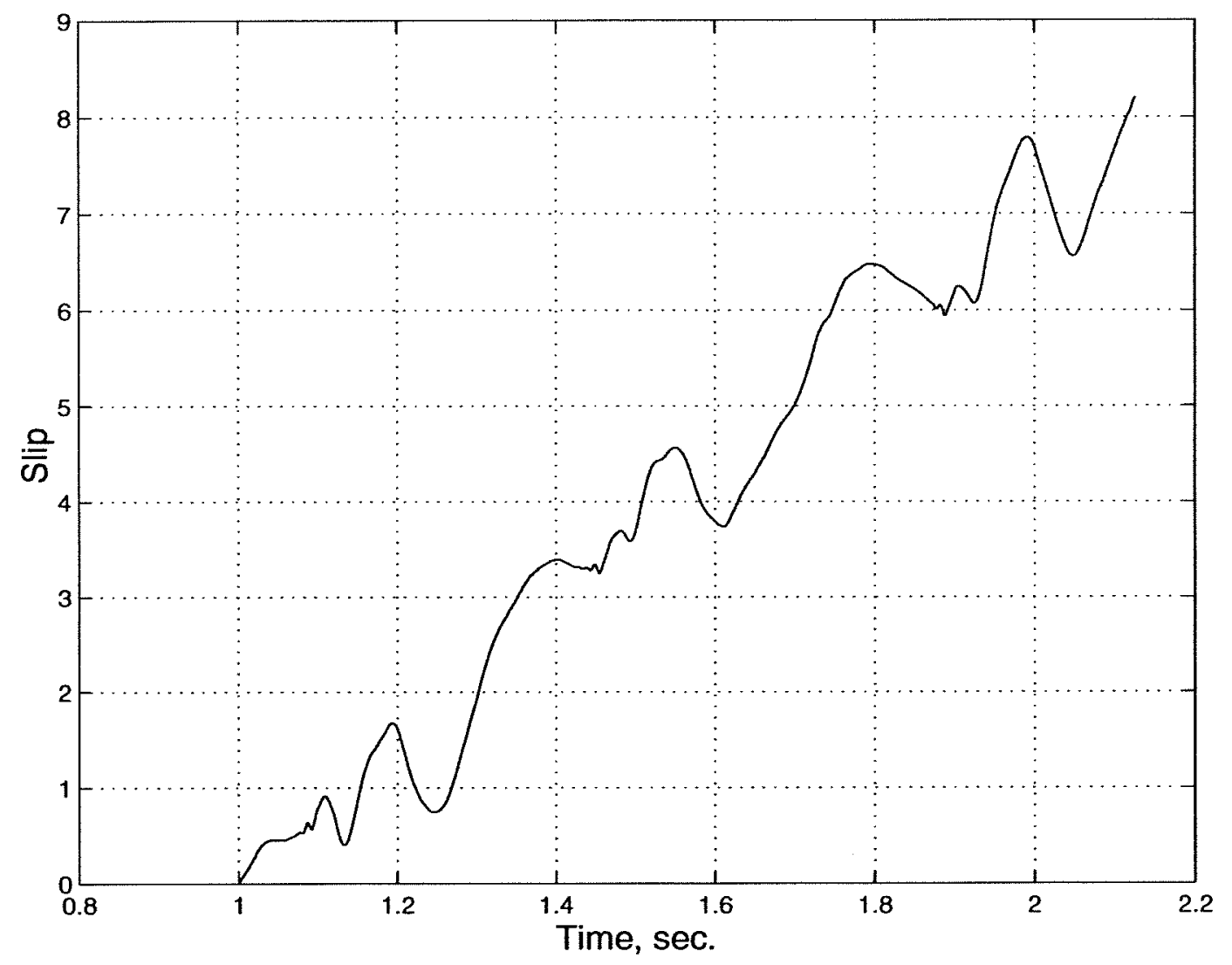

Figure 6-15: Slip function for part of the time series shown on Fig. 6-13. Note the pattern characteristic of stick-slip motion, when the process of slipping stops for a time (or even reverses) and then continues further on. 

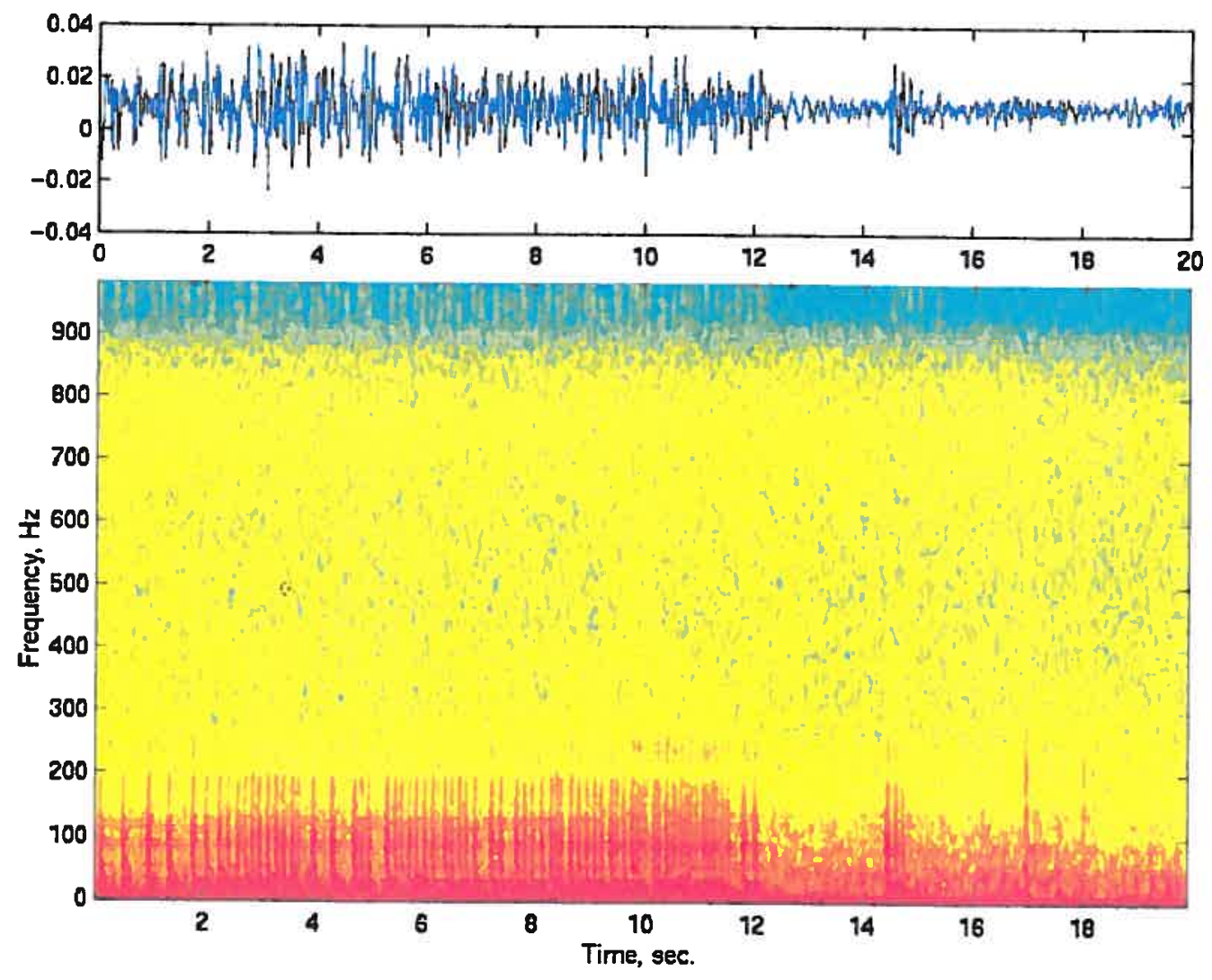

Figure 6-16: Spectrogram of vertical velocity on geophone \#2 at the beginning of the tape RLAM-26. Note that the regular stick-slip pattern of the spectrum changes after about $12 \mathrm{sec}$. 
When two surfaces of a brittle material are placed together, the asperities on the surfaces in contact become locked together. If the normal load is high enough to prevent the surfaces from lifting up over the irregularities, then sliding will occur when the locked regions fail brittlely.

The evidence for the type of motion (stick-slip) described above was found in the geophone data from the ice island experimental site. On Fig. 6-13 about $1.5 \mathrm{sec}$ of the time series of the vertical component of velocity on geophone \#2 are shown. Note several pulses similar in shape at approximately $1.1 \mathrm{sec}, 1.4 \mathrm{sec}$ and $1.8 \mathrm{sec}$ on Fig. 6-13(a) which are characteristic for a stick-slip type of motion. Fig. 6-13(b) shows the details of the pulse at $1.4 \mathrm{sec}$ which indicate the flexural wave arrival. The autocorrelation function (see Fig. 6-14) confirms the similarity between the pulses on Fig. 6-13. Fig. 6-15 depicts the slip function for the time series shown on Fig. 613. Note the pattern characteristic of stick-slip motion, when the process of slipping stops for some time (or even reverses) and then continues further on. On Fig. 6-16 the spectrogram of vertical velocity on one geophone is shown: observe how the regular stick-slip pattern changes after about 12 sec.

\subsection{Event parameter estimation from the combined results of spectral processing and slip history determination}

\subsubsection{Event parameter definitions}

As it was mentioned above, the two most important source parameters characterizing an ice event are seismic moment $M_{0}$ and stress drop $\Delta \sigma$. According to [22], the seismic moment $M_{0}$ is defined as

$$
M_{0}=\mu \bar{u} A,
$$




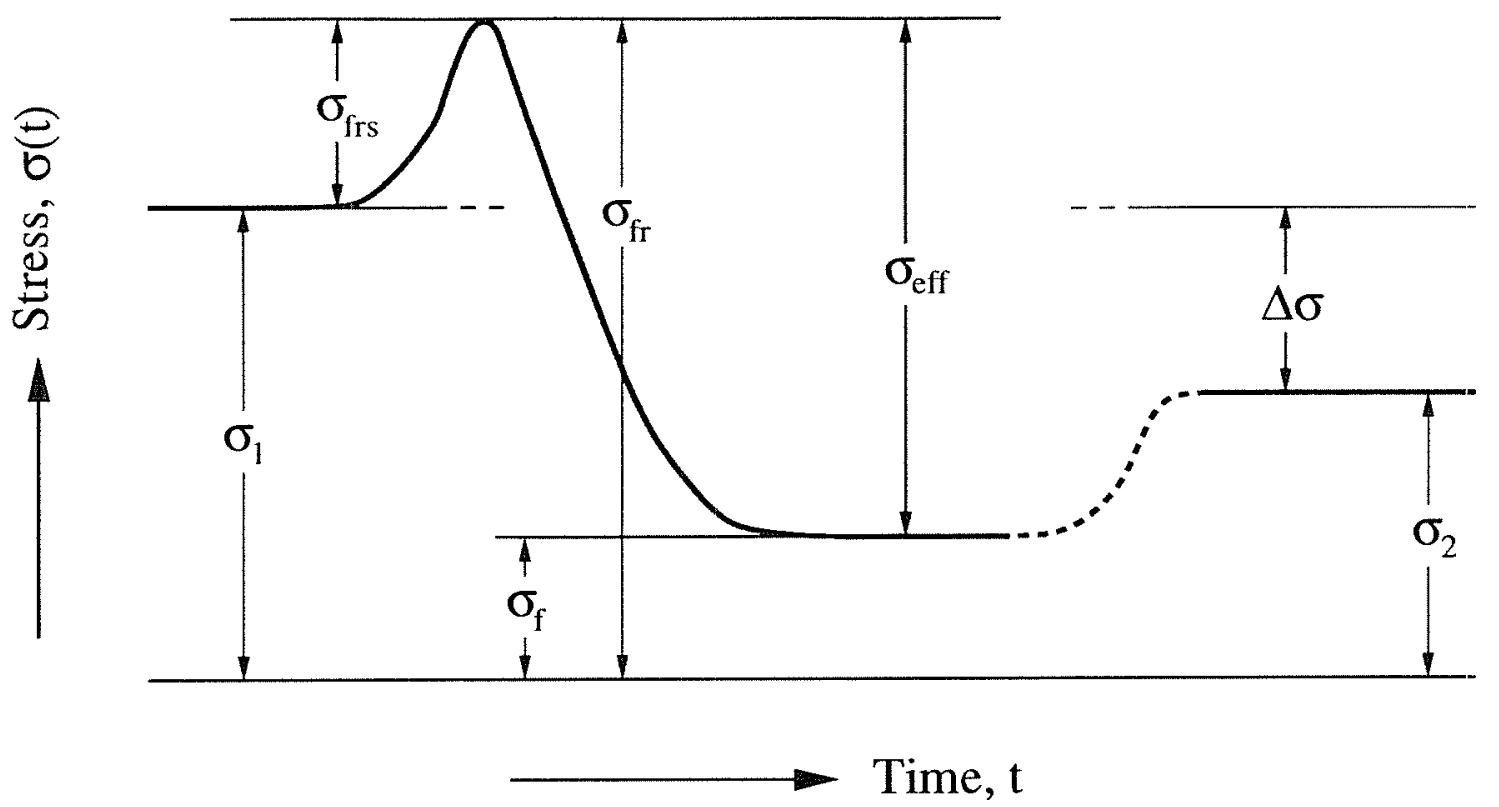

Figure 6-17: Stress drop definition according to [21]. Here $\sigma_{1}$ is the initial stress, $\sigma_{2}$ is the final stress, $\sigma_{\mathrm{eff}}$ is the effective stress, $\sigma_{f}$ is the dynamic frictional stress, and $\sigma_{f r}$ is the static frictional stress. The stress drop, $\Delta \sigma$, refers to the difference between $\sigma_{1}$ and $\sigma_{2}$.

where:

$\mu$-shear strength of ice,

$\bar{u}$ - average slip,

$A$ - fault area.

To determine the stress drop in an ice event we need to consider the stress change in time during the faulting process according to [21] (see Fig. 6-17). In order for the rupturing to begin the local stress must rise by at least $\sigma_{\mathrm{frs}}$ (the difference between the frictional stress and initial stress). A slip occurs in those sections of fault where this condition is satisfied. On this figure the stress drop, $\Delta \sigma$, is defined as the difference between the initial stress, $\sigma_{1}$, and the final stress, $\sigma_{2}$. The other, often used parameter, effective stress $\sigma_{\text {eff }}$, denotes the difference between static and dynamic friction. Sometimes, instead of stress drop people use fractional stress drop $\varepsilon$ that is defined as the ratio of stress drop to the effective stress available for fracturing:

$$
\varepsilon=\frac{\Delta \sigma}{\sigma_{\mathrm{eff}}} .
$$


According to [46] the relation between average stress drop and slip is given by:

$$
\Delta \sigma=C \mu\left(\frac{\bar{u}}{L}\right),
$$

where

$$
C= \begin{cases}\frac{2}{\pi} & \text { for a strike-slip fault } \\ \frac{4(\lambda+\mu)}{\pi(\lambda+2 \mu)} & \text { for a dip-slip fault }\end{cases}
$$

In Equation (6.6) $\bar{u}$ is again the average slip, and $L$ is the characteristic rupture dimension.

\subsubsection{Procedure for event parameter estimation}

In Equations (6.5) and (6.6) all parameters on the right-hand side can be estimated using the results from spectral and slip history methods. To show how this can be done, let us recall from the previous discussion that, according to [3], the corner frequency is inversely related to the dimensions of the source (or source duration $T$ ):

$$
f_{0}=1 / \pi T .
$$

Now, to obtain the length of a crack from its duration $T$ we need to make an assumption regarding the rupture velocity $v_{r}$. According to [31]

$$
v_{r} \approx 0.63 c_{s},
$$

where $c_{s}$ is the shear wave speed. So we can obtain the length of a crack $L$ using the above value of rupture velocity:

$$
L=\frac{v_{r}}{\pi f_{0}} .
$$

A value of $\rho=920 \mathrm{~kg} / \mathrm{m}^{3}$ for the density of sea ice is used for calculations 
based on data provided by [38]:

The density of pure ice is $916.8 \mathrm{~kg} / \mathrm{m}^{3}$. However the density of sea-ice may be greater than this last figure (if brine is trapped among the ice crystals) or less (if the brine has escaped and gas bubbles are present). Values from 924 to $857 \mathrm{~kg} / \mathrm{m}^{3}$ were recorded on the Norwegian Maud Expedition.

The values of other important constants for ice are given below:

$$
\begin{aligned}
c_{s} & =1800 \mathrm{~m} / \mathrm{s} \\
\mu & =3 \times 10^{9} \mathrm{~Pa}
\end{aligned}
$$

So finally, using Equation (6.8) and (6.9) and values of the constants for ice, we can estimate the event parameters from the following relations:

$$
\begin{aligned}
& M_{0}=\mu \bar{u} A=\mu \bar{u} \frac{v_{r} h}{\pi f_{0}} \\
& \Delta \sigma=C \mu \frac{\bar{u}}{L}=C \mu \bar{u} \frac{\pi f_{0}}{v_{r}}
\end{aligned}
$$

where $h$ is the ice thickness $(2 \mathrm{~m})$. So, we see that $M_{0}$ and $\Delta \sigma$ can be estimated using only results from processing by both spectral and slip history determination methods and values of appropriate physical constants for ice.

\subsubsection{The results of estimation of event parameters and com- parison with previous estimates}

In Table 6.1 results of parameter estimation using both spectral and slip history determination methods and Equations (6.10)-(6.11) are given for several events from the beginning of tape RLAM-26. In this table, $T_{0}$ denotes approximate arrival time

for the event in seconds from the beginning of tape RLAM-26, $\bar{u}$ is the average slip (after correction for range spreading), $f_{0}$ is the corner frequency, $M_{0}$ is the seismic moment, and $\Delta \sigma$ is the average stress drop. 


\begin{tabular}{||c|c|c|r|c|c||}
\hline Event \# & $T_{0}, \mathrm{sec}$ & $\bar{u}, \mathrm{~mm}$ & $f_{0}, \mathrm{~Hz}$ & $M_{0}, \mathrm{MNm}$ & $\Delta \sigma, \mathrm{kPa}$ \\
\hline 3 & 37 & 0.037 & 7.8 & 10.4 & 1.54 \\
5 & 39 & 0.043 & 8.8 & 10.6 & 2.00 \\
9 & 58 & 0.029 & 8.4 & 7.4 & 1.27 \\
10 & 65 & 0.011 & 7.5 & 3.1 & 0.42 \\
15 & 145 & 0.057 & 7.0 & 17.6 & 2.09 \\
16 & 169 & 0.056 & 7.9 & 15.3 & 2.34 \\
\hline
\end{tabular}

Table 6.1: Event parameters for several events at the beginning of tape RLAM-26. Note that units of seismic moment $M_{0}$ are $\mathrm{MNm}$ (i.e. $10^{6} \mathrm{~N} \times \mathrm{m}$ ).

These results give the following range for stress drop values: $\Delta \sigma=0.42-$ $-2.34 \mathrm{kPa}$. The range of values for seismic moment is $M_{0}=3.1--17.6 \times 10^{6} \mathrm{Nm}$.

In the thesis of C. Stamoulis [49], stress drop ranges from $110 \mathrm{~Pa}$ at $f=300 \mathrm{~Hz}$ to $2.25 \times 10^{4} \mathrm{~Pa}$ at $f=40 \mathrm{~Hz}$ which is at least one order of magnitude higher than my upper limit of values for stress drop. For most of the events processed by C. Stamoulis in [49] the estimates of seismic moment $M_{0}$ lie in between $10^{7}$ and $10^{10} \mathrm{Nm}$. Only very few events actually go below $10^{7} \mathrm{Nm}$ value, but nevertheless some overlap between my estimates of $M_{0}$ and those of [49] could be observed.

To compare with Chen's [4] results one needs to be aware of the arithmetical error on page 105 of [4]: when plugging values in Equation (4.42) on that page the actual numerical coefficient obtained is $9.9 \times 10^{-4}$, not $5.9 \times 10^{-4}$, as written in text. Hence all subsequently derived relations should be multiplied by factor 1.68 to correct for that error. In further discussion, first, the result obtained using the wrong value will be shown, then, in parenthesises, the result using the correct value will be shown.

The stress drop range is predominantly from 10 to $1000 \mathrm{~Pa}$. The lowest frequency data point is at approximately $70 \mathrm{~Hz}$ with a value of stress drop being $\Delta \sigma=5 \mathrm{kPa}$.

After adapting notation, Chen's regression formula for the dependence of stress drop on frequency (Equation (4.44) on page 105 of [4]) takes the form

$$
\Delta \sigma=2.7 \times 10^{4} f^{-1 \pm 0.47}
$$


(the correct coefficient should be 4.6 instead of 2.7 ).

According to that relation for frequency $f=10 \mathrm{~Hz}$ we have $\Delta \sigma=2.7 \mathrm{kPa}$ $(4.6 \mathrm{kPa})$. So the corrected value, $4.6 \mathrm{kPa}$, is slightly higher than the upper limit of my range of values for $\Delta \sigma(2.34 \mathrm{kPa})$.

To estimate the seismic moment Chen uses an octopole strength and obtains the following expression for the frequency dependence of the seismic moment (Equation (4.45) on page 106 of [4]):

$$
M_{0}=4.1 \times 10^{9} f^{-2.5}
$$

(the correct coefficient should be 6.9 instead of 4.1). Note that the above-discussed error propagates into the results for the seismic moment.

That should give us the following value for the seismic moment at $f=10 \mathrm{~Hz}$ : $M_{0}=13.0 \times 10^{6} \mathrm{Nm}$ (actually, $21.8 \times 10^{6} \mathrm{Nm}$ ). Again, the corrected value is slightly higher than the upper limit of my range of values of the seismic moment $(17.6 \times$ $10^{6} \mathrm{Nm}$ ), but taking into account the variation of Chen's data, one can say that her estimates lie within the range of event parameters estimated in this thesis.

Note, that the ice fracture strength lies in the range between $2 \times 10^{5}$ and $1 \times 10^{6} \mathrm{~Pa}$. For both Chen's estimates and my own, the difference between the stress drop in ice events and the ice fracture strength is at least two orders of magnitude. For Stamoulis' estimates this difference is at least one order of magnitude. This is somewhat similar to what is happening in seismology: in the case of earthquakes, there is usually a difference of 2-4 orders of magnitude between measured stress drops and the shear strength of rock.

\subsection{Conclusions}

Using spectral methods of event processing, a high frequency trend rolling off as $\omega^{-3}$ in the vertical displacement spectra of events was found which probably could be attributed to fault nucleation. In some of the events at even higher frequencies 
a second trend, $\omega^{-2}$, was observed. Such frequency behavior is consistent with a commonly accepted model in earthquake seismology for high frequency behavior of the displacement spectrum [21].

The results of source parameter inversion give the following ranges of values:

Stress drop: $\Delta \sigma=0.42--2.34 \mathrm{kPa}$.

Seismic moment: $M_{0}=3.1--17.6 \times 10^{6} \mathrm{Nm}$.

These results are quite close to estimates by Chen for the marginal ice zone, but somewhat different from those by C. Stamoulis for the same Arctic region, which probably indicates that physical conditions on the "ice island" (where all the data processed in this chapter were collected) are closer to that of the marginal ice zone than to those usually characteristic of the Central Arctic. The other possibility is that the kind of events observed on the "ice island" was different from those in the vicinity of the main surveillance array which was the area covered in [49]. The low level of pressure on the lone hydrophone accompanying the RLAM unit during the ice events discussed above seems to confirm that possibility.

The estimates of stress drop are at least two orders of magnitude lower than the ice fracture strength value which is similar to earthquake mechanics case (2-4 orders of magnitude). This fact probably indicates that the events occured on the preexisting fault surfaces. 


\section{Chapter 7}

\section{Conclusions and suggestions for future work}

\subsection{Conclusions}

Using the Motion Product Detector method for processing multi-component geophone data, the polarization characteristics of elastic waves were determined. The fractures processed by this method seemed to generate vertically polarized shear (SV) waves for the most part. Their concentration in the vicinity of the corner of the new ice ridge and the character of the particle motion in the corresponding arrivals indicate the continuing ridge building process in that part of the ice island, which is located approximately $4 \mathrm{~km}$ East of the base camp.

The processing of data from an ice lead approximately $2 \mathrm{~km}$ North of the base camp provided evidence for the existence of a new phenomenon: edge waves, waves propagating along a newly opened lead. The waves exhibit a quasi-periodic behavior suggesting some kind of stick-slip generation mechanism somewhere along the length of the lead. The propagation characteristics of these waves were determined using seismic wavenumber estimation techniques. In the low-frequency limit the dispersion can be modeled approximately by an interaction at the lead edges of the lowest order, antisymmetric modes of the infinite plate.

The qualitative comparison of theoretical radiation patterns with radiation 
characteristics of ice events suggests a strike-slip fault with a dip angle of $90^{\circ}$ as the most probable source mechanism for the ice event processed here, which is somewhat to the contrary of J.S. Kim's conclusion [22]. On the basis of the relative smallness of the radiation from longitudinal wave compared to the acoustic mode he suggested that dip-slip with dip angle $\delta \approx 0^{\circ}$ and $90^{\circ}$ and strike-slip with dip angle $\delta \approx 0^{\circ}$ are the most probable source mechanisms. This discrepancy may arise from the fact that most of the events processed here do not radiate efficiently into the water.

Using spectral methods, the high frequency trend $\omega^{-3}$ in the vertical displacement spectra of events was found which probably could be attributed to fault nucleation processes. In some of the events at even higher frequencies a second trend, $\omega^{-2}$, was observed. That one corresponds to the high frequency behavior of the displacement spectrum that is found in the commonly accepted model from earthquake seismology.

The result of the source parameter inversion gives the following ranges of values:

Stress drop: $\Delta \sigma=0.42--2.34 \mathrm{kPa}$.

Seismic moment: $M_{0}=3.1--17.6 \times 10^{6} \mathrm{Nm}$.

These results are quite close to estimates by Chen for the marginal ice zone, but somewhat different from those by C. Stamoulis for the same Arctic region, which probably indicates that physical conditions on the ice island (where most of the data processed in this thesis were collected) were closer to that of the marginal ice zone than to those usually characteristic of the Central Arctic. The other possibility is that the kinds of events observed on the ice island were different from those in the vicinity of main surveillance array which was the area covered in [49]. This difference could be due to the active ridge building processes on the ice island which were absent near base camp during the time period covered by the data processing in Stamoulis' thesis [49]. The low level of pressure on the lone hydrophone accompanying the RLAM unit during the above discussed ice events seems to confirm that possibility. 
The estimates for stress drop are at least two orders of magnitude lower than the ice fracture strength value which is similar to the case of earthquake mechanics (2-4 orders of magnitude). This fact probably indicates that the events occured on the preexisting fault surfaces.

\subsection{Suggestions for future work}

Additional modeling is required to understand more completely the nature of the new 'edge wave' phenomenon described above, in particular in regard to achieving a better match of the dispersion behavior with the model.

The other direction for future work is a more extensive use of polarization methods from earthquake seismology for geophone data processing. 


\section{Appendix A}

\section{Inverse for an overconstrained}

\section{system}

When there are more observations than unknown model parameters $(n>m)$ the solution, if it exists, should be determined by a least squares method. Let the error for each data set be $\mathbf{e}$.

$$
\mathbf{e}=\mathbf{A x}-\mathbf{y}
$$

The squared norm is obtained from a product of $\mathbf{e}^{\mathrm{T}}$ and $\mathbf{e}$ :

$$
\begin{aligned}
e^{2} & =\mathbf{e}^{\mathrm{T}} \mathbf{e}=(\mathbf{A} \mathbf{x}-\mathbf{y})^{\mathrm{T}}(\mathbf{A} \mathbf{x}-\mathbf{y}) \\
& =\left(\mathbf{x}^{\mathrm{T}} \mathbf{A}^{\mathrm{T}}-\mathbf{y}^{\mathrm{T}}\right)(\mathbf{A} \mathbf{x}-\mathbf{y})=\mathbf{x}^{\mathrm{T}} \mathbf{A}^{\mathrm{T}} \mathbf{A} \mathbf{x}-\mathbf{y}^{\mathrm{T}} \mathbf{A} \mathbf{x}-\mathbf{x}^{\mathrm{T}} \mathbf{A}^{\mathrm{T}} \mathbf{y}+\mathbf{y}^{\mathrm{T}} \mathbf{y}
\end{aligned}
$$

To minimize the squared norm we differentiate in a vector space with respect to $\mathbf{x}$ or $\mathrm{x}^{\mathrm{T}}$ and set the result equal to zero:

$$
\begin{aligned}
\frac{\partial e^{2}}{\partial \mathbf{x}} & =\mathbf{x}^{\mathrm{T}} \mathbf{A}^{\mathrm{T}} \mathbf{A}-\mathbf{y}^{\mathrm{T}} \mathbf{A}=0 \\
\frac{\partial e^{2}}{\partial \mathbf{x}^{\mathrm{T}}} & =\mathbf{A}^{\mathrm{T}} \mathbf{A} \mathbf{x}-\mathbf{A}^{\mathrm{T}} \mathbf{y}=0 .
\end{aligned}
$$


Both equations yield the same result, so the solution for the estimate, $\mathrm{x}^{\mathrm{b}}$, could be obtained from the Equation (A.3):

$$
\begin{gathered}
{\left[\mathbf{A}^{\mathrm{T}} \mathbf{A}\right] \mathbf{x}^{\mathrm{b}}=\mathbf{A}^{\mathrm{T}} \mathbf{y}} \\
\mathbf{x}^{\mathrm{b}}=\left[\mathbf{A}^{\mathrm{T}} \mathbf{A}\right]^{-1} \mathbf{A}^{\mathrm{T}} \mathbf{y} .
\end{gathered}
$$

It is necessary that the matrix $\left[\mathrm{A}^{\mathrm{T}} \mathrm{A}\right]$ be non-singular:

$$
\operatorname{det}\left(\mathbf{A}^{\mathrm{T}} \mathbf{A}\right) \neq 0 .
$$

The matrix $\left[\mathbf{A}^{\mathrm{T}} \mathbf{A}\right]$ is obviously an $m \times m$ autocovariance matrix, while $\mathbf{A}^{\mathrm{T}} \mathbf{y}$ is a cross covariance. 


\section{Bibliography}

[1] K. Aki and P.G. Richards. Quantitative seismology. Theory and Methods. W.H. Freeman and Company, New York, 1980.

[2] W.F. Brace and J.D. Byerlee. Stick-slip as a mechanism for earthquakes. Science, 153:990-992, 1966.

[3] J.N. Brune. Tectonic stress and the spectra of seismic shear waves from earthquakes. Journal of Geophysical Research, 75(26):4997-5009, 1970.

[4] C.F. Chen. Analysis of Marginal Ice Zone Noise Events. PhD thesis, Massachusetts Institute of Technology, Cambridge, MA, 1990.

[5] H.-I. Choi and W.J. Williams. Improved time-frequency representation of multicomponent signals using exponential kernels. IEEE Transactions on Acoustics, Speech and Signal Processing, 37(6):862-871, 1989.

[6] Yu. Dudko, H. Schmidt, K. von der Heydt, and E.K. Scheer. Edge wave observation using remote seismoacoustic sensing of ice events in the Arctic. JGR, 103(C10):21775-21781, 1998.

[7] I. Dyer. The song of sea ice and other Arctic Ocean melodies. In I. Dyer and C. Chryssostomidis, editors, Arctic Policy and Technology, pages 11-37. Hemisphere Publishing Co., 1983.

[8] I. Dyer. Speculations on the origin of low frequency Arctic ocean noise. In B. Kerman, editor, Sea Surface Sound: Natural Mechanisms of Surface Gener- 
ated Noise in the Ocean, pages 513-532. NATO Advanced Research Workshop, Lerici, Italy, 1987.

[9] A. Dziewonski, S. Bloch, and M. Landisman. A technique for the analysis of transient seismic signals. Bulletin of the Seismological Society of America, 59(1):427444, February 1969.

[10] D.M. Farmer and Yunbo Xie. The sound generated by propagating cracks in sea ice. $J A S A, 85(4): 1489-1500$, April 1989.

[11] C.A.J. Fletcher. Computational Galerkin methods. Springer-Verlag, New York, 1984.

[12] J.H. Ganton and A.R. Milne. Temperature- and wind-dependent ambient noise under midwinter pack ice. Journal of the Acoustical Society of America, 38:406411, Feb. 1965.

[13] D.C. Gazis and R.D. Mindlin. Extensional vibrations and waves in a circular disk and a semi-infinite plate. Journ. Appl. Mech., 27:541-547, 1960.

[14] R.V. Goldstein, A.V. Marchenko, and A.Yu. Semenov. Edge waves in the fluid under the elastic plate with a crack. Doklady Akademii Nauk (in Russian), 339(3):331-334, 1994.

[15] R.D. Gregory and I. Gladwell. The reflection of a symmetric Raleigh-Lamb wave at the fixed or free edge of a plate. Journal of Elasticity, 13:185-206, 1983.

[16] N.A. Haskell. Total energy and energy spectral density of elastic wave radiation from propagating faults. Bulletin of the Seismological Society of America, 54:1811-1841, 1964.

[17] S. Haykin, editor. Array Signal Processing. Prentice Hall signal processing series. Prentice Hall, Englewood Cliffs, New Jersey, 1985.

[18] K. Hunkins. Seismic studies of sea ice. Journal of Geophysical Research, $65(10): 3459-3472,1960$. 
[19] A. Jurkevics. Polarization analysis of three-component array data. BSSA, 78(5):1725-1743, October 1988.

[20] T. Kapoor. Detection and characterization of active ice fracture events during SIMI-94 experiments. Technical report, Massachusetts Institute of Technology, November 1995. Progress report.

[21] K. Kasahara. Earthquake mechanics. Cambridge earth science series. Cambridge University Press, Cambridge, UK, 1981.

[22] J.S. Kim. Radiation from directional seismic sources in laterally stratified media with application to Arctic ice cracking noise. PhD thesis, Massachusetts Institute of Technology, Cambridge, MA, May 1989.

[23] A.J. Langley. Exact and approximate theories for fluid-loaded, infinite, elastic plates. Journal of the Acoustical Society of America, 83:1366-1375, 1988.

[24] A.J. Langley. The sound fields of an infinite, fluid-loaded plate excited by a point source. Journal of the Acoustical Society of America, 83(4):1360-1365, Apr. 1988.

[25] A.J. Langley. Acoustic emission from the arctic ice sheet. Journal of the Acoustical Society of America, 85(2):692-701, Feb. 1989.

[26] J.K. Lewis and W.W. Denner. Higher frequency ambient noise in the Arctic ocean. Journal of the Acoustical Society of America, 84(4):1444-1455, Oct. 1988.

[27] J.K. Lewis, P.J. Stein, and W.W. Denner. Stress relief in sea ice as related to the generation of higher frequency noise in the arctic ocean. Technical Report SAIC-89/1101, Science Applications International Corp., 1989.

[28] R. Madariaga. Dynamics of seismic sources. In E.S. Husebye and S. Mykkeltveit, editors, Identification of Seismic Sources - Earthquake or Underground Explosion, pages 71-96. D. Reidel Publishing Company, Oslo, Norway, September 1980. 
[29] N.C. Makris and I. Dyer. Environmental correlates of pack ice noise. Journal of the Acoustical Society of America, 79(5):1434-1440, May 1986.

[30] N.C. Makris and I. Dyer. Environmental correlates of Arctic ice-edge noise. Journal of the Acoustical Society of America, 90(6):3288-3298, 1991.

[31] L. Mansinha. The velocity of shear fracture. Bulletin of the Seismological Society of America, 54:369-376, Feb. 1964.

[32] B.E. Miller. Observation and inversion of seismo-acoustic waves in a complex arctic ice environment. Ms thesis, Massachusetts Institute of Technology, Cambridge, MA, September 1990.

[33] B.E. Miller and H. Schmidt. Observation and inversion of seismo-acoustic waves in a complex arctic ice environment. Journal of the Acoustical Society of America, 89(4):1668-1685, April 1991.

[34] A.R. Milne. Thermal tension cracking in sea ice: A source of underice noise. Journal of Geophysical Research, 77(12):2177-2192, Apr. 1972.

[35] A.R. Milne and J.H. Ganton. Ambient noise under arctic sea ice. Journal of the Acoustical Society of America, 36(5):855-863, Feb. 1964.

[36] A.R. Milne, J.H. Ganton, and D.J. McMillin. Ambient noise under sea ice and further measurements of wind and temperature dependence. Journal of the Acoustical Society of America, 41:525-528, Feb. 1967.

[37] R.D. Mindlin. Introduction to the Mathematical Theory of Vibrations of Elastic Plates. U.S.Army Signal Corps, 1955.

[38] G.L. Pickard and W.J. Emery. Descriptive physical oceanography: an introduction. Pergamon Press, Oxford, England, 5th enlarged edition (in SI units) edition, 1990.

[39] F. Press and M. Ewing. Propagation of elastic waves in a floating ice sheet. Transactions, American Geophysical Union, 32(5):673-678, 1951. 
[40] R.S. Pritchard. Arctic Ocean background noise caused by ridging of sea ice. Journal of the Acoustical Society of America, 75(2):419-427, Feb. 1984.

[41] R. Sato. Study on surface waves II. Velocity of surface waves propagated upon elastic plates. Bulletin of Earthquake Research Inst. of Tokyo Univ., 29:223-261, 1951.

[42] R. Sato. Analysis of dispersed waves by means of Fourier transformation I. Bulletin of Earthquake Research Inst. of Tokyo Univ., 33:33-48, 1955.

[43] R. Sato. Formulations of solutions for earthquake source models and some related problems. Journal of Physics of the Earth, 17(2):101-110, 1969.

[44] H. Schmidt. OASES: Version 2.1. User guide and reference manual. Technical report, Massachusetts Institute of Technology, Cambridge, MA, May 1997.

[45] H. Schmidt, A.B. Baggeroer, I. Dyer, J.R. Fricke, and Y.P. Guo. Acoustical aspects of Sea-Ice Mechanics sea ice fracture inversion. Proposal for theoretical and field research, Massachusetts Institute of Technology, Cambridge, MA, 1991.

[46] C.H. Scholz. The mechanics of earthquakes and faulting. Cambridge University Press, Cambridge, UK, 1990.

[47] E.A.G. Shaw. On the resonant vibrations of thick barium titanate disks. Journal of the Acoustical Society of America, 28(2):38-50, 1956.

[48] P. Silver. Retrieval of source-extent parameters and the interpretation of corner frequency. Bulletin of the Seismological Society of America, 73(6):1499-1511, Dec. 1983.

[49] C. Stamoulis. Analysis of Ice-Induced Acoustic Events in the Central Arctic. $\mathrm{PhD}$ thesis, Massachusetts Institute of Technology, Cambridge, MA, September 1997.

[50] P.J. Stein. Acoustic monopole in a floating ice plate. $\mathrm{PhD}$ thesis, Massachusetts Institute of Technology, Cambridge, MA, Feb. 1986. 
[51] P.J. Torvik. Reflection of wave trains in semi-infinite plates. Journal of the Acoustical Society of America, 41(2):346-353, 1967.

[52] K. von der Heydt, H. Schmidt, and A.B. Baggeroer. Measurement and inversion of acoustic emission from ice-mechanical processes in the Arctic. Proposal for field experiment and data analysis, WHOI, Woods Hole, MA, 1992.

[53] J.E. White. Motion product seismograms. Geophysics, 29(2):288-298, April 1964.

[54] T.C. Yang and G.R. Giellis. Experimental characterization of elastic waves in a floating ice sheet. Journal of the Acoustical Society of America, 96(5):2993-3009, Nov. 1994.

[55] T.C. Yang and T.W. Yates. Flexural waves in a floating ice sheet: modeling and comparison with data. Journal of the Acoustical Society of America, 97(2):971977, 1995. 